\title{
REPORT ON A 2009 MINI-DEMONSTRATION OF THE ARG-US RADIO FREQUENCY IDENTIFICATION (RFID) SYSTEM IN TRANSPORTATION
}

\section{Conducted for}

The DOE Packaging Certification Program of U.S. Department of Energy Environmental Management, Office of Packaging and Transportation

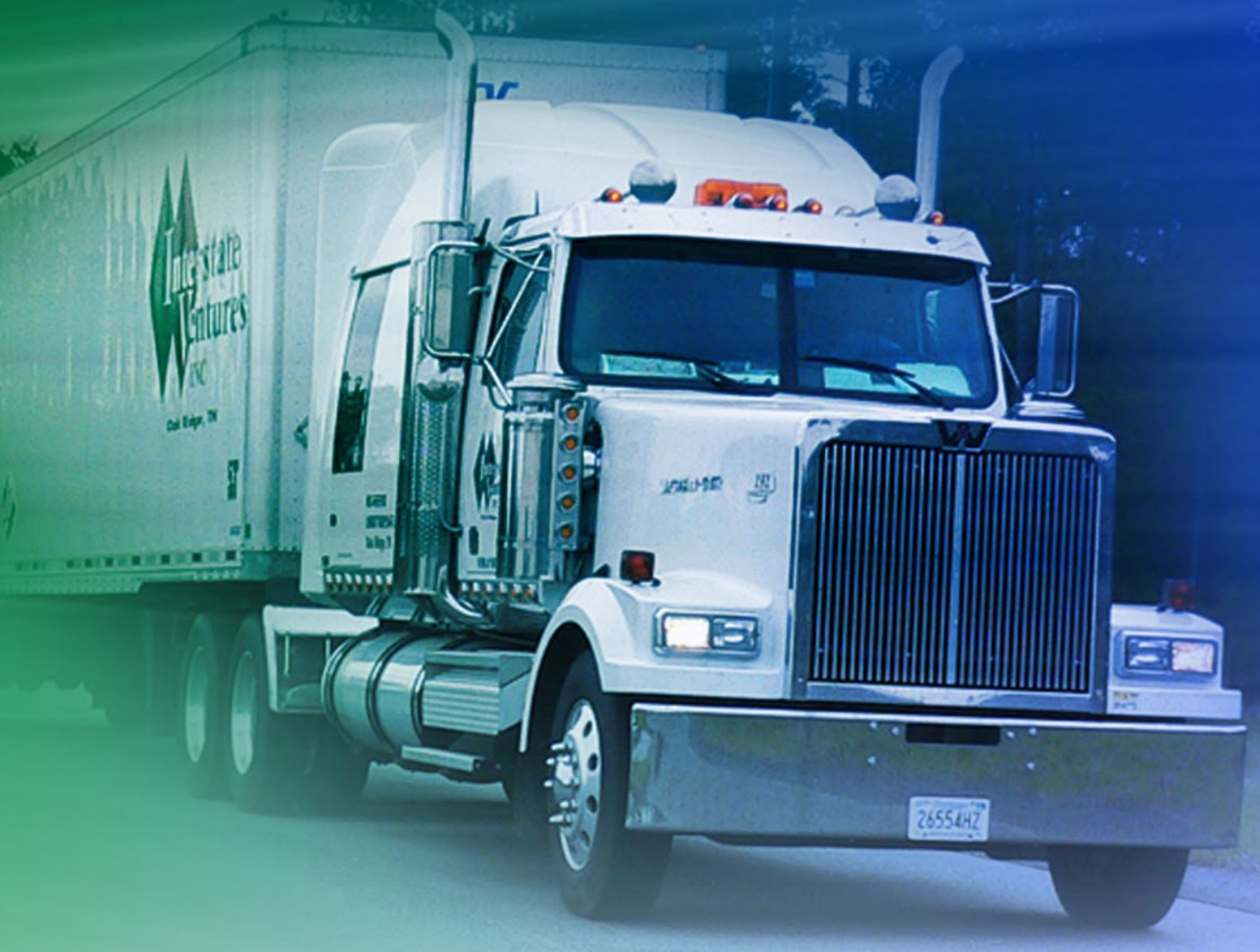

The mission of the Packaging Certification Program (PCP) is to ensure the safety and security of packagings for radioactive and fissile materials and support vital DOE missions across the DOE Complex, as well as EM's risk reduction, clean up and site closure activities. 


\begin{abstract}
About Argonne National Laboratory
Argonne is a U.S. Department of Energy laboratory managed by UChicago Argonne, LLC under contract DE-AC02-06CH11357. The Laboratory's main facility is outside Chicago, at 9700 South Cass Avenue, Argonne, Illinois 60439. For information about Argonne and its pioneering science and technology programs, see www.anl.gov.
\end{abstract}

\title{
Availability of This Report
}

This report is available, at no cost, at http://www.osti.gov/bridge. It is also available on paper to the U.S. Department of Energy and its contractors, for a processing fee, from:

U.S. Department of Energy

Office of Scientific and Technical Information

P.O. Box 62

Oak Ridge, TN 37831-0062

phone (865) 576-8401

fax (865) 576-5728

reports@adonis.osti.gov

\begin{abstract}
Disclaimer
This report was prepared as an account of work sponsored by an agency of the United States Government. Neither the United States Government nor any agency thereof, nor UChicago Argonne, LLC, nor any of their employees or officers, makes any warranty, express or implied, or assumes any legal liability or responsibility for the accuracy, completeness, or usefulness of any information, apparatus, product, or process disclosed, or represents that its use would not infringe privately owned rights. Reference herein to any specific commercial product, process, or service by trade name, trademark, manufacturer, or otherwise, does not necessarily constitute or imply its endorsement, recommendation, or favoring by the United States Government or any agency thereof. The views and opinions of document authors expressed herein do not necessarily state or reflect those of the United States Government or any agency thereof, Argonne National Laboratory, or UChicago Argonne, LLC.
\end{abstract}




\section{Report on a 2009 Mini-Demonstration of the ARG-US Radio Frequency Identification (RFID) System in Transportation}

by

Hanchung Tsai, Kun Chen, Mark Jusko, Brian Craig, and Yung Liu

Decision and Information Sciences Division, Argonne National Laboratory

for

The DOE Packaging Certification Program of U.S. Department of Energy

Environmental Management, Office of Packaging and Transportation

September 30, 2009 


\section{Table of Contents}

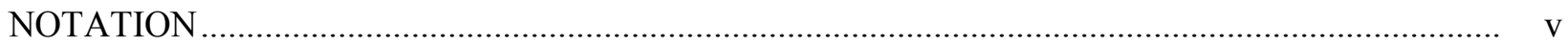

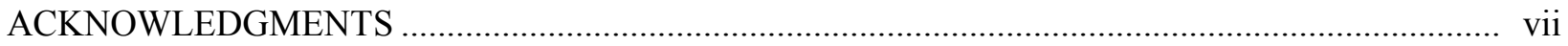

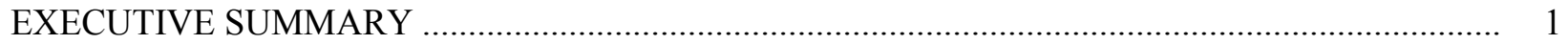

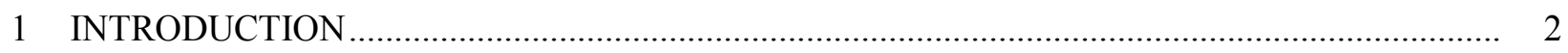

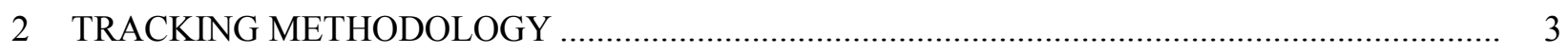

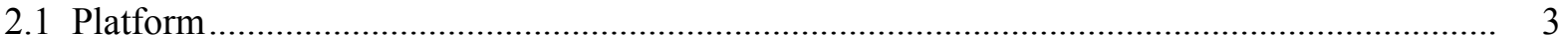

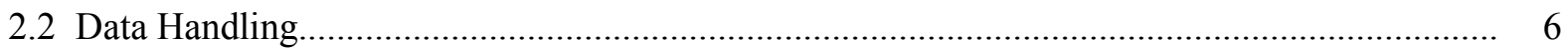

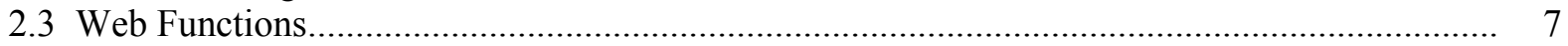

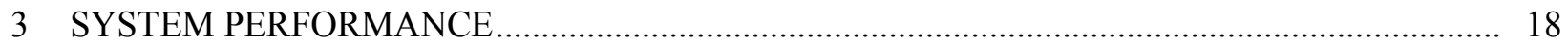

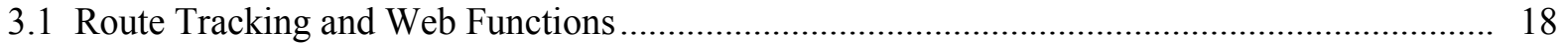

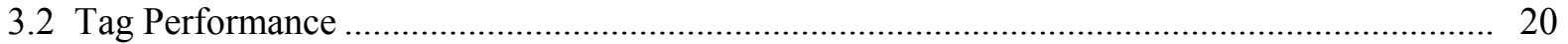

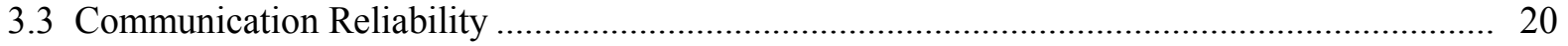

3.4 System Performance - Staged Incidents …..................................................................... 21

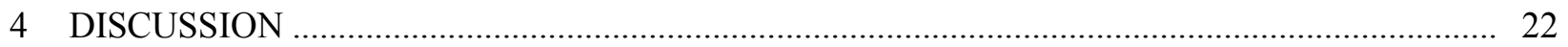

4.1 Integration of RFID and Qualcomm Components ................................................................. 22

4.2 Need for Enhanced RFID Reader Performance …............................................................... 22

4.3 Need for Two-Way Communication between Reader in Truck and Command Center.............. 22

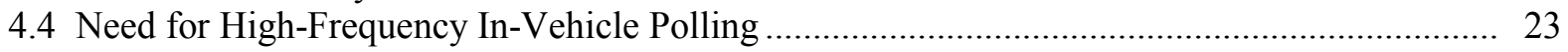

4.5 Further Enhancing Web Page Functionality................................................................. 23

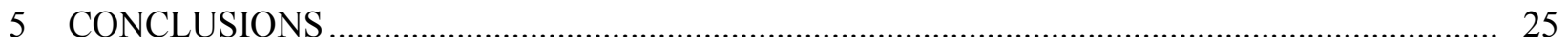

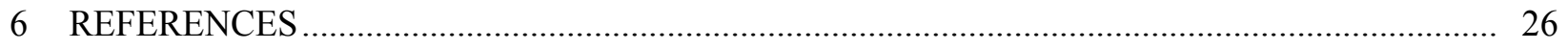

Appendices

A. Example 1: A Free-Form Text (FFT) Message …............................................................. 27

Example 2: A Byte Array (shown in Hex) Sent Over-the-Air ............................................. 27

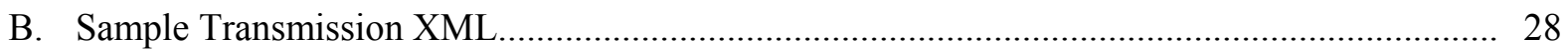

C. Summary Recorded Data from the MiniDemo ................................................................... 29

D. Geographic Information System (GIS) Reports ........................................................... 54 


\section{Figures}

1 Schematic of Qualcomm's OmniTRACS system (Courtesy of Qualcomm Inc.) ........................... 3

2 Schematic diagram of communication platform used in the MiniDemo........................................ 4

3 Mk-II RFID tag (top) and mounting of tags on multiple drum types ............................................ 4

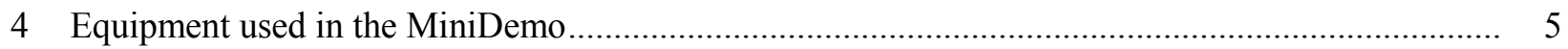

5 Planned route for the MiniDemo and the locations for the six staged incidents ............................ 18

6 Actual route traveled ("bread-crumbing") in the MiniDemo ........................................................ 19

7 Summary status of tags, as captured by a screenshot ............................................................. 19

8 Time lags between sent (from vehicle) and received (at Qualcomm Hub). Lags for staged incidents were typically smaller, as the message is brief and take precedent in the send priority.

\section{Tables}

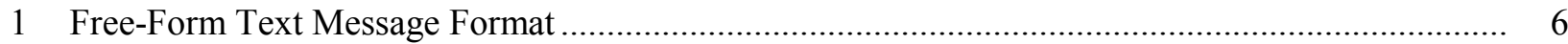

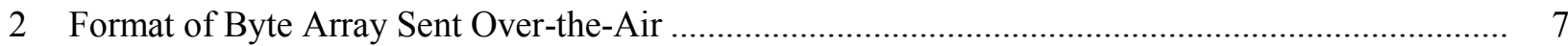




\section{Notation}

\section{Acronyms}

\begin{tabular}{|c|c|}
\hline ARG-US & Integrated hardware and software sets from Argonne \\
\hline ASCII & American Standard Code for Information Interchange \\
\hline CCITT & $\begin{array}{l}\text { Consultative Committee for International Telegraphy and Telephony } \\
\text { (now ITU-T) }\end{array}$ \\
\hline $\mathrm{CRC}$ & Cylindrical redundancy check \\
\hline DEMO & Demonstration \\
\hline DIS & Tracking system developed by Argonne \\
\hline DOE & U.S. Department of Energy \\
\hline DOE TRANS-COM & Tracking system \\
\hline EM & Environmental Management \\
\hline EM-45 & Office of Packaging and Transportation \\
\hline ESRI & Environmental Systems Research Institute \\
\hline FFT & Free form text \\
\hline GeoLogic & Tracking system \\
\hline GIS & Geographic information system \\
\hline GMT & Greenwich Mean Time \\
\hline HEX & Hexadecimal \\
\hline IMCT & Integrated mobile communication terminal \\
\hline ITU-T & International Telecommunication Union (formerly CCITT) \\
\hline $\mathrm{MHz}$ & Megahertz \\
\hline MIP & Mobile interface protocol \\
\hline NMF & Network management facility \\
\hline OmniTRACS & Integrated hardware and software sets from Qualcomm \\
\hline PCP & Packaging Certification Program \\
\hline RFID & Radio frequency identification \\
\hline URL & Uniform resource locator \\
\hline VP & Vehicle position \\
\hline VTS & Tracking system developed by Argonne \\
\hline XML & Extensible markup language \\
\hline
\end{tabular}




\section{Units of Measure}

$\begin{array}{ll}\mathrm{h} & \text { hour(s) } \\ \mathrm{mi} & \operatorname{mile}(\mathrm{s})\end{array}$




\section{Acknowledgments}

The authors wish to acknowledge Dr. James Shuler, Manager of the DOE Packaging Certification Program (PCP), Office of Packaging and Transportation (EM-45), for his continuing support and guidance of the project on applying the Radio Frequency Identification (RFID) technology to the management of nuclear materials. The authors also wish to thank Bob Eddy, Tony Wingfield, and Ed Thompson of Qualcomm - without their generosity and assistance, the tasks described in the report would not have been possible. 


\title{
Report on a 2009 Mini-Demonstration of the ARG-US Radio Frequency Identification (RFID) System in Transportation
}

\author{
Executive Summary
}

The Packaging Certification Program (PCP) of the U.S. Department of Energy (DOE) Environmental Management (EM), Office of Packaging and Transportation (EM-14), has developed a radio frequency identification (RFID) tracking and monitoring system for the management of nuclear materials during storage and transportation. The system, developed by the PCP team at Argonne National Laboratory, consists of hardware (Mk-series sensor tags, fixed and handheld readers, form factor for multiple drum types, seal integrity sensors, and enhanced battery management), software (application programming interface, ARG-US software for local and remote/web applications, secure server and database management), and cellular/satellite communication interfaces for vehicle tracking and item monitoring during transport.

The ability of the above system to provide accurate, real-time tracking and monitoring of the status of multiple, certified containers of nuclear materials has been successfully demonstrated in a week-long, 1,700-mile DEMO performed in April 2008. While the feedback from the approximately fifty (50) stakeholders who participated in and/or observed the DEMO progression were very positive and encouraging, two major areas of further improvements - system integration and web application enhancement - were identified in the post-DEMO evaluation. The principal purpose of the MiniDemo described in this report was to verify these two specific improvements. The MiniDemo was conducted on August 28, 2009.

In terms of system integration, a hybrid communication interface - combining the RFID itemmonitoring features and a commercial vehicle tracking system by Qualcomm — was developed and implemented. In the MiniDemo, the new integrated system worked well in reporting tag status and vehicle location accurately and promptly. There was no incompatibility of components. The robust commercial communication gear, as expected, helped improve system reliability. The MiniDemo confirmed that system integration is technically feasible and reliable with the existing RFID and Qualcomm satellite equipment.

In terms of web application, improvements in mapping, tracking, data presentation, and post-incident spatial query reporting were implemented in ARG-US, the application software that manages the dataflow among the RFID tags, readers, and servers. These features were tested in the MiniDemo and found to be satisfactory. The resulting web application is both informative and user-friendly.

A joint developmental project is being planned between the PCP and the DOE TRANSCOM that uses the Qualcomm gear in vehicles for tracking and communication of radioactive material shipments across the country. Adding an RFID interface to TRANSCOM is a significant enhancement to the DOE infrastructure for tracking and monitoring shipments of radioactive materials. 


\section{Introduction}

In April 2008, the DOE Packaging Certification Program (PCP), at the direction of Dr. Ines Triay, the Assistant Secretary of the Office of Environment Management, conducted a demonstration (DEMO) of the application of radiofrequency identification (RFID) technology for tracking and monitoring of nuclear materials containers during storage and transportation. The DEMO, conducted by the PCP team at Argonne National Laboratory, used a rental semi-trailer and fourteen (14) certified containers (i.e., drums) for nuclear materials, each fitted with an active RFID tag equipped with a suite of sensors for temperature, humidity, shock, seal integrity, and battery status. The drums (Model 9975, 9977, and ES3100) were empty, but otherwise the DEMO represented the operating conditions in the real-world environment, including loading and unloading of pallets of drums from the trailer and incidents and alarm notifications along the route and at the stopover facilities. Four vehicle tracking systems - DIS, VTS, DOE TRANSCOM, and GeoLogic - were deployed during the 4.5-day DEMO. Two of the systems, DIS and VTS, developed by Argonne, additionally monitored the status of the tagged drums in the trailer. RFID tag monitoring was not possible for DOE TRANSCOM and GeoLogic during the DEMO because of a lack of an interface with the RFID reader in the truck. These two government/commercial systems nonetheless provided a valuable side-by-side comparison with the Argonne's developmental systems during the DEMO (Tsai et al. 2008).

In the post-DEMO evaluation, it was recognized that the robust hardware and infrastructure features of the government/commercial systems could be used, at relatively low cost, to enhance the efficacy of the PCP RFID tracking and monitoring system, and vice versa. Both DOE TRANSCOM and GeoLogic systems proved to be more reliable than Argonne's developmental systems in reporting vehicle locations because of their vehicle-mounted antennas and the backup satellite communication options; neither were available for Argonne's developmental systems. Further, both DOE TRANSCOM and GeoLogic have dedicated data/communication centers established solely for tracking and monitoring transportation vehicles. Other tangible assets of the government/commercial systems include structure and interfaces associated with user groups, training programs, web/mapping infrastructure, data security provisions, and - most importantly - maturity from years of operations. Of the two, DOE TRANSCOM is preferred for integration with the PCP RFID system because it is already being used for DOE's "high-visibility" radioactive materials and waste shipments. (Over 12,000 shipments have been tracked by TRANSCOM since 2001.)

DOE TRANSCOM uses on-board GPS satellite equipment provided by Qualcomm, Inc. This MiniDemo was conducted principally to study potential compatibility issues between Argonne's RFID system and the Qualcomm/TRANSCOM system. A set of Qualcomm's tracking equipment, called "OmniTRACS," and twenty-five (25) Mk-I and -II RFID tags, a reader, and a control computer were used in the MiniDemo. The tags were not mounted on drums because of the lack of a full complement of such drums at Argonne. However, from the standpoint of tag performance or satellite communication, the test results are valid whether the tags are mounted or not. 


\section{Tracking Methodology}

\subsection{Platform}

The in-vehicle components of OmniTRACS include a keyboard/display unit (tablet) and an externalmounted multidirectional satellite antenna communication unit (Qualcomm 2004). Together, they are called "Integrated Mobile Communication Terminal" (IMCT). In operation, the in-vehicle system communicates with the U.S. GPS satellites for position information and the Data Satellite for data and message exchanges (Figure 1). In the implementation of the RFID interface, the added tag information would be transmitted as part of the message to the Data Satellite and be received at Qualcomm/TRANSCOM for processing. In the MiniDemo, the "Command Center" was Argonne's secure server.

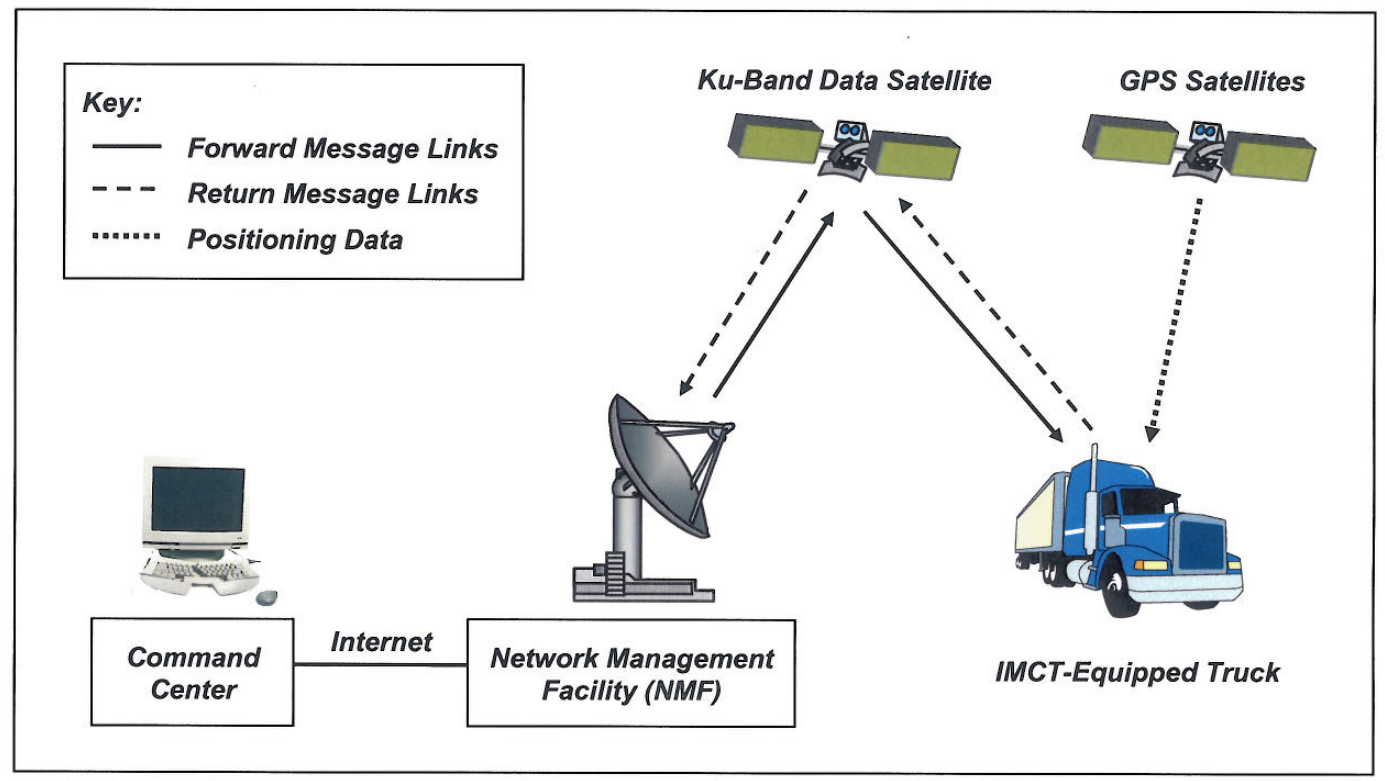

FIGURE 1 Schematic of Qualcomm's OmniTRACS system (Courtesy of Qualcomm Inc.)

The communication platform used in the MiniDemo is an integration of the PCP's RFID system, called "ARG-US," and Qualcomm's OmniTRACS system. The interplay of the systems components is shown in Figure 2.

The Mk-I and -II tags (Chen et al. 2009) are equipped with sensors for seal integrity, temperature, humidity, shock, and battery status. They are also equipped with non-volatile memories for storing the content manifest and the sensor event data. The alarm thresholds for the sensors are adjustable to suit the specific mode of operation (e.g., transport or storage). The seal sensors of all tags were compressed simulating the mounted state of tags on drums. Figure 3 shows the front and interior views of an Mk-II tag and actual mounting of tags on multiple drum types. 


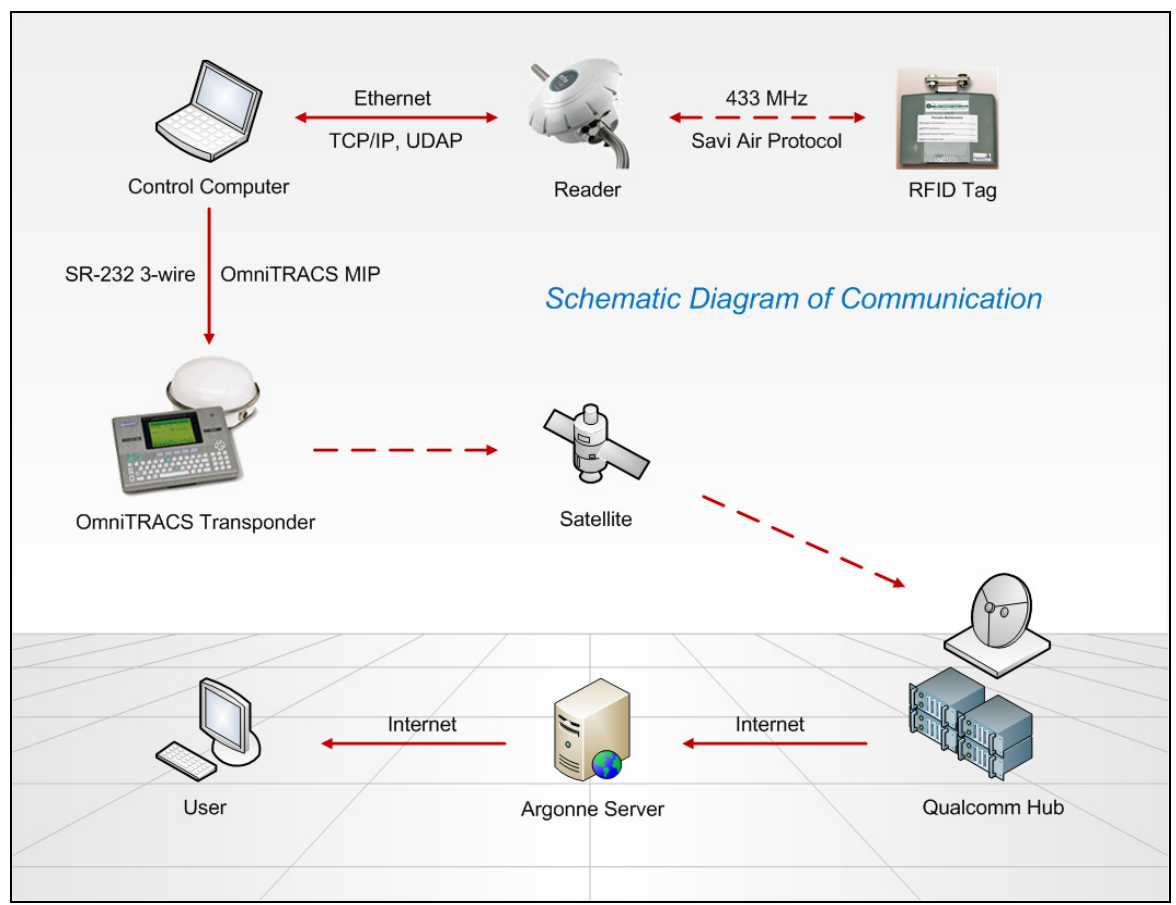

FIGURE 2 Schematic diagram of communication platform used in the MiniDemo.

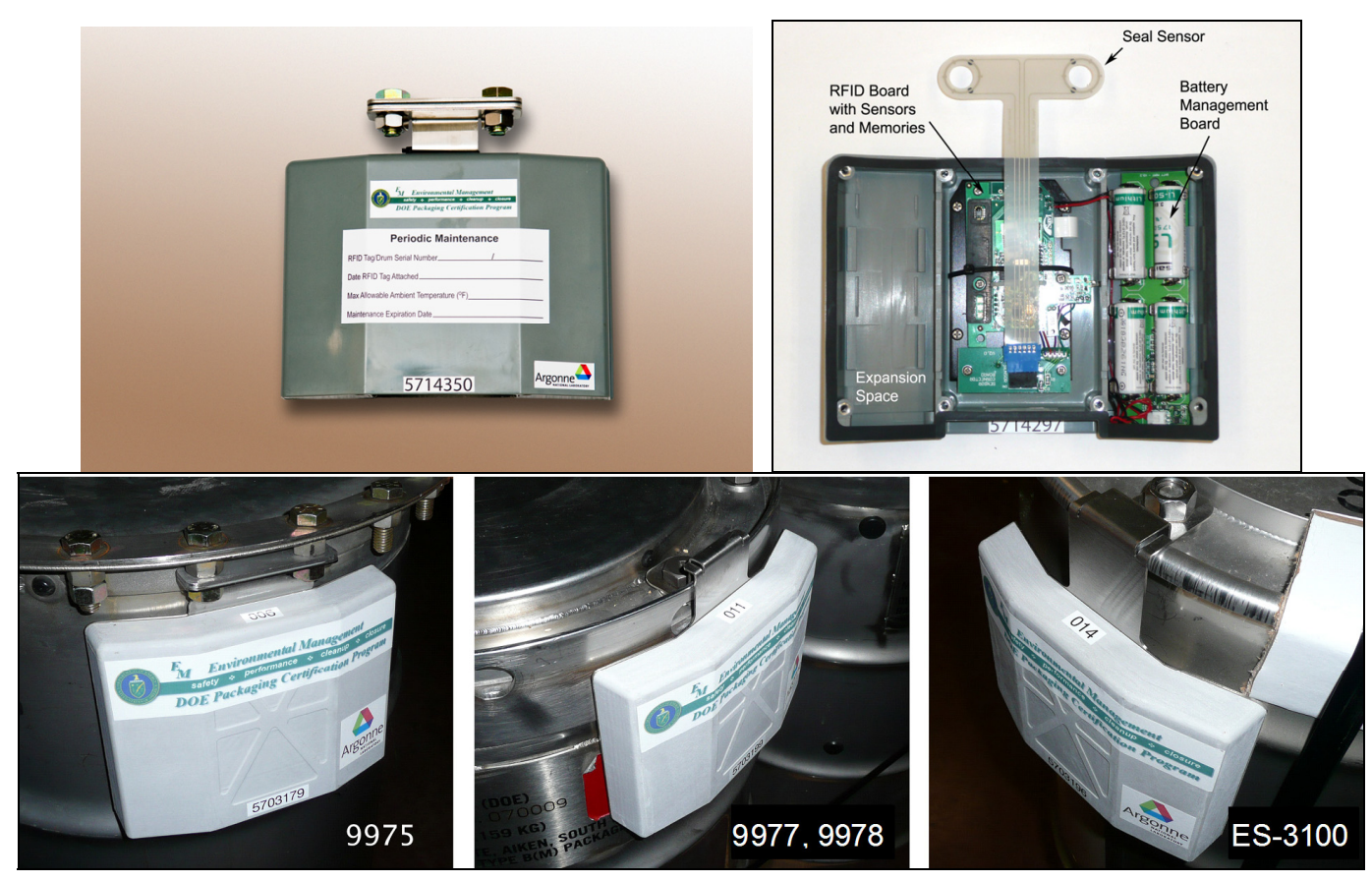

FIGURE 3 Mk-II RFID tag (top) and mounting of the tags on multiple drum types. 
The MiniDemo was performed on August 28, 2009 with a rental vehicle driven from Argonne to East Moline, IL, on I-80 and back to Argonne on I-88 for a total of $\approx 300$ miles in slightly less than 6.5 hours.

Figure 4 shows the vehicle, the rooftop-mounted multidirectional satellite antenna communication unit, and the tablet display unit by Qualcomm, as well as the Mk-II RFID tags and the reader in the vehicle.
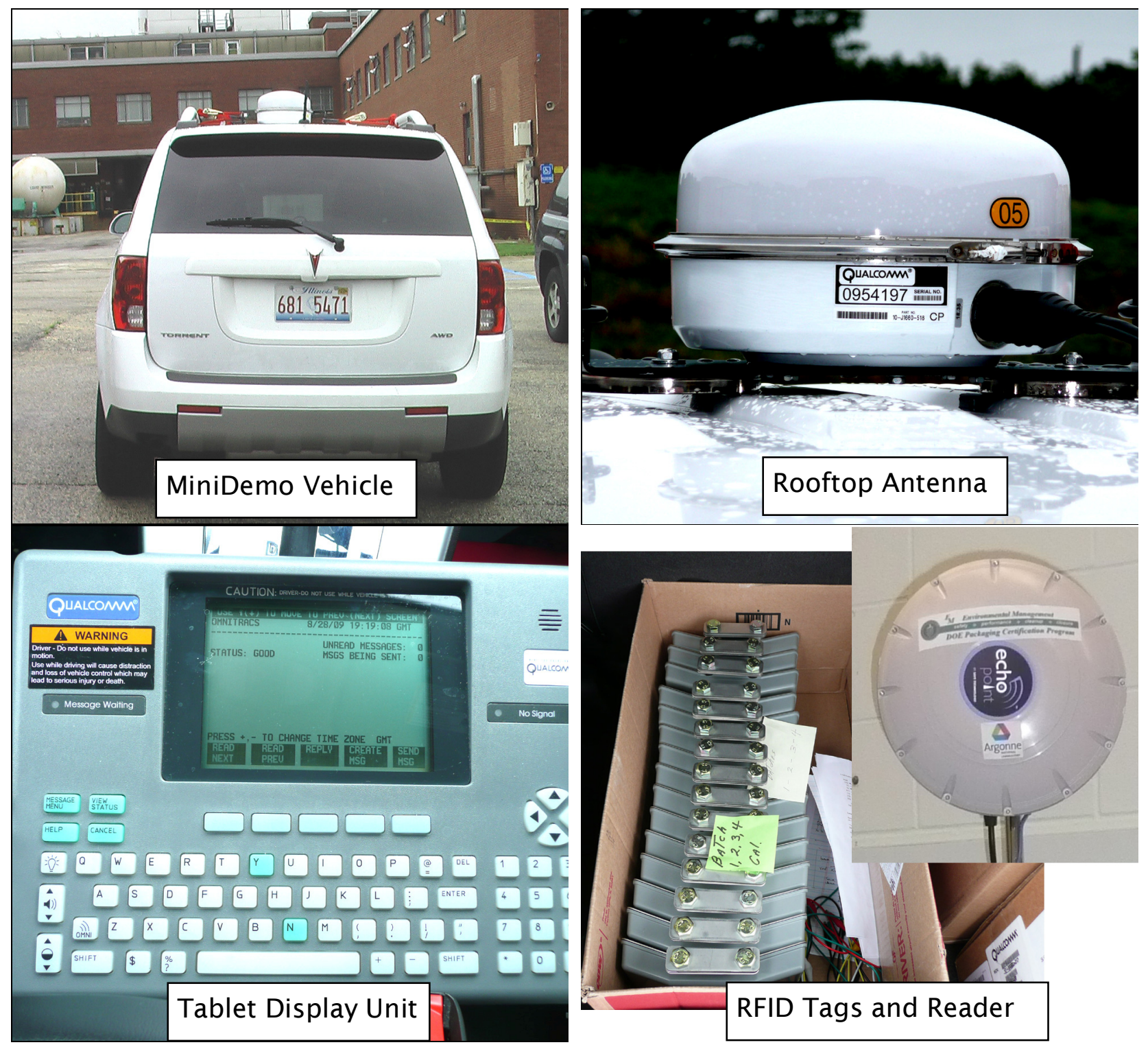

FIGURE 4 Equipment used in the MiniDemo. 


\subsection{Data Handling}

Inside the MiniDemo vehicle, the reader polls the 25 RFID tags by 433-MHz radio waves and relays the collected data through an Ethernet cable to a data processor. Polling frequency, which is adjustable, was set at 5 minutes. Between pollings, the tags can be assumed to be in a battery-power-conserving, halfasleep state. When any of the sensor alarm thresholds in the tag are exceeded, the tag wakes up instantly and sends an alarm message to the reader. The alarm message takes precedence over all other actions that the reader is undertaking at that time.

The purpose of the data processor (at present, served by a laptop computer, Figure 2) is to format the collected tag data for efficient transmission to the satellite via the Qualcomm Display Unit ("tablet") and antenna (Figure 4). When the conditions of the drums are normal, this transmission takes place after each polling (i.e., every 5 minutes). If a tag-generated alarm message is received by the reader, that message takes priority and is sent first. The sending of the regular data packet to the satellite is resumed after the alarm message is sent.

For each message sent, the current time is added to the sensor information. The time stamp and tag information are formatted in a Free Form Text (FFT) message per the Qualcomm mobile interface protocol (MIP). The format is user-defined and shown in Table 1. The FFT message is then converted to a byte array that can be sent out via a 3-wire serial port in the tablet display unit and the OmniTRACS antenna. The format of the array is defined by Qualcomm and shown in Table 2. All message formation and conversion are done by the data processor. Sample FFT messages and byte arrays are given in Appendix A.

TABLE 1 Free Form Text Message Format

\begin{tabular}{ll}
\hline Position & \multicolumn{1}{c}{ Content } \\
\hline & \\
1 & Format Flat (0 plain text, 1 compressed) \\
$2-15$ & Date/Time (MMDDYYYYHHmSS) \\
$16-26$ & Tag ID (text padded on right with spaces) \\
$27-28$ & Event Code (HEX) \\
29 & Seal Code (HEX) \\
$30-31$ & Seal Value (HEX) \\
32 & Temperature Code (HEX) \\
$33-34$ & Temperature Value (HEX) \\
35 & Humidity Code (HEX) \\
$36-37$ & Humidity Value (HEX) \\
38 & Shock Code (HEX) \\
$39-40$ & Shock Value (HEX) \\
41 & Radiation Code (HEX) \\
$42-43$ & Radiation Value (HEX) \\
44 & Battery Code (HEX) \\
& Repeat the above for the next tag until all tags are processed \\
\hline
\end{tabular}

Note: The Free Form Text (FFT) message format is user-defined. The maximum length of the message is 1900 bytes. The FFT supports only 6-bits ASCII. 
When the FFT message is sent from the vehicle, a Vehicle Position (VP) message is also generated and sent. The latest position, as well as the nearest cities/towns and a date/time stamp, are contained in the VP message. From the data satellite, the message is relayed to the Qualcomm Network Management Facility (NMF) or Qualcomm Hub, as depicted in Figures 1 and 2, respectively. The VP messages are important as they allow the Argonne RFID server to obtain the current latitude and longitude information of the vehicle.

TABLE 2 Format of Byte Array Sent Over-the-Air

\begin{tabular}{lll}
\hline \multicolumn{1}{c}{ Message } & \multicolumn{1}{c}{ Length } & \multicolumn{1}{c}{ Note } \\
\hline END & 1 byte & $\mathrm{C} 0$ \\
SPARE 1 & 1 byte & 00 \\
PACKET TYPE & 1 byte & $07=$ text message \\
SEQUANCE \# & 1 byte & 00 \\
CONTROL & 1 byte & $00=$ no error \\
ERROR & 1 byte & $00=$ no error \\
RESERVED & 1 byte & 00 \\
DATA 0 & 1 byte & $00=$ Normal message \\
FFT MESSAGE & 0 - 1900 bytes & ASCII 6 bits only \\
CRC high byte & 1 byte & High byte of CRC-16-CCITT \\
CRC low byte & 1 byte & Low byte of CRC-16-CCITT \\
END & 1 byte & C0
\end{tabular}

Note: A Cylindrical Redundancy Check (CRC) is used to ensure data integrity. The generating polynomial is the standard CCITT (now ITU-T) polynomial used in X.25 protocol. The seed value of the $\mathrm{CRC}$ is $0 \mathrm{xFFFF}$. The byte array that is used for calculating the final value of CRC starts from "SPARE1" and ends at the end of the FFT message.

At the RFID command center (Bldg. 900 at Argonne for the MiniDemo), the RFID software continually polls the Qualcomm Hub at 1-minute intervals to check if any new transmissions have been received. When a transmission is returned, the software converts the message from the transmission encoding of Base 64 into an Extensible Markup Language (XML) collection of transmissions (see an example in Appendix B). After the message is converted, the software parses the XML to find the formatted text that represents the transmitted RFID tag data (see Appendix C). Once the text has been located, the software parses the formatted text and inserts the latest sensor values into the RFID database. At this point, the newly obtained data are shown on the RFID website.

\subsection{Web Functions}

Significant improvements in web functions have been made since the April 2008 DEMO. One of the major emphases is improved mapping. Planned route, route tracking (bread-crumbing), zooming, and spot information are some of the new features added. The ability to view detailed information on tags/drums in tabulated form for the current and past time steps is now available directly on the webpage, without having to navigate through the server database. Another important improvement is in geographic information system (GIS) reporting - pre-formatted GIS reports, when warranted, can now be issued with a single click from the Command Center. GIS reports, with a concise summary of local assets and 
vulnerabilities, are important for the first responders and emergency management in case of a transportation incident.

The current RFID web site's URL is https://rfid.dis.anl.gov/pcp-test/webform1.aspx. After opening the page, the first transportation vehicle, if any exist, will become the selected location. The web page always displays information about the currently selected location. There are seven major sections on the RFID web page, as delineated below.

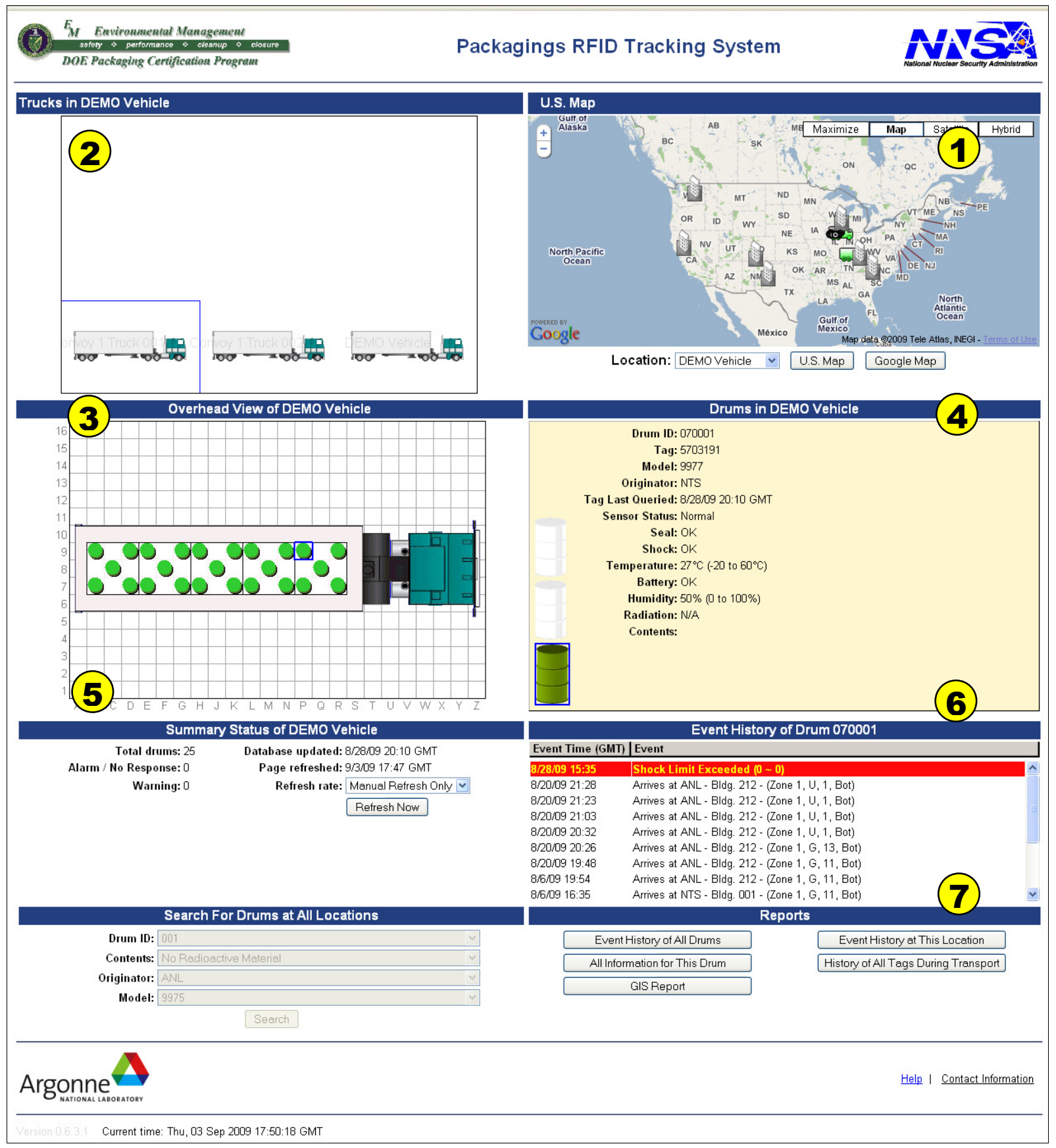


(1)

By default, the first section of the main web page contains a Google map of the United States showing all PCP RFID implementation locations, whether they are storage sites or transportation vehicles. Users can pan the map by dragging the "hand" cursor back and forth. Various details about this selected location are shown throughout the page. One way to change the location is by clicking on its icon or using the drop-down menu:

\begin{tabular}{|c|l|}
\hline Location: & \multicolumn{2}{|l|}{ DEMO Vehicle } \\
& $\checkmark$ \\
\hline & DEMO Vehicle \\
& Virtual Truck 1 \\
\hline ANL Bldg. 212 \\
LANL Bldg. 001 \\
LLNL Bldg. 001 \\
RL Bidg. 001 \\
SRS BIdg. 001 \\
SRS Bldg. 002 \\
WIPP Bldg. 001 \\
Y-12 Bldg. 001 \\
\hline
\end{tabular}

The color of each location's icon represents the current status of sensors in the RFID tags, indicating the "state of health" of the monitored drums:

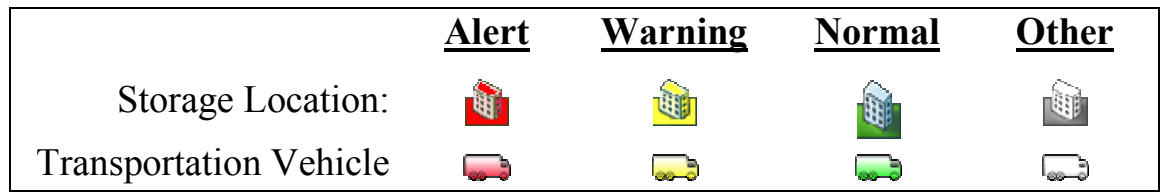

If the Maximize button on the Google map is clicked when a transportation vehicle is selected, the web page will change. A larger map will be displayed, along with a table at the bottom detailing the chronological status of all drums in that vehicle:

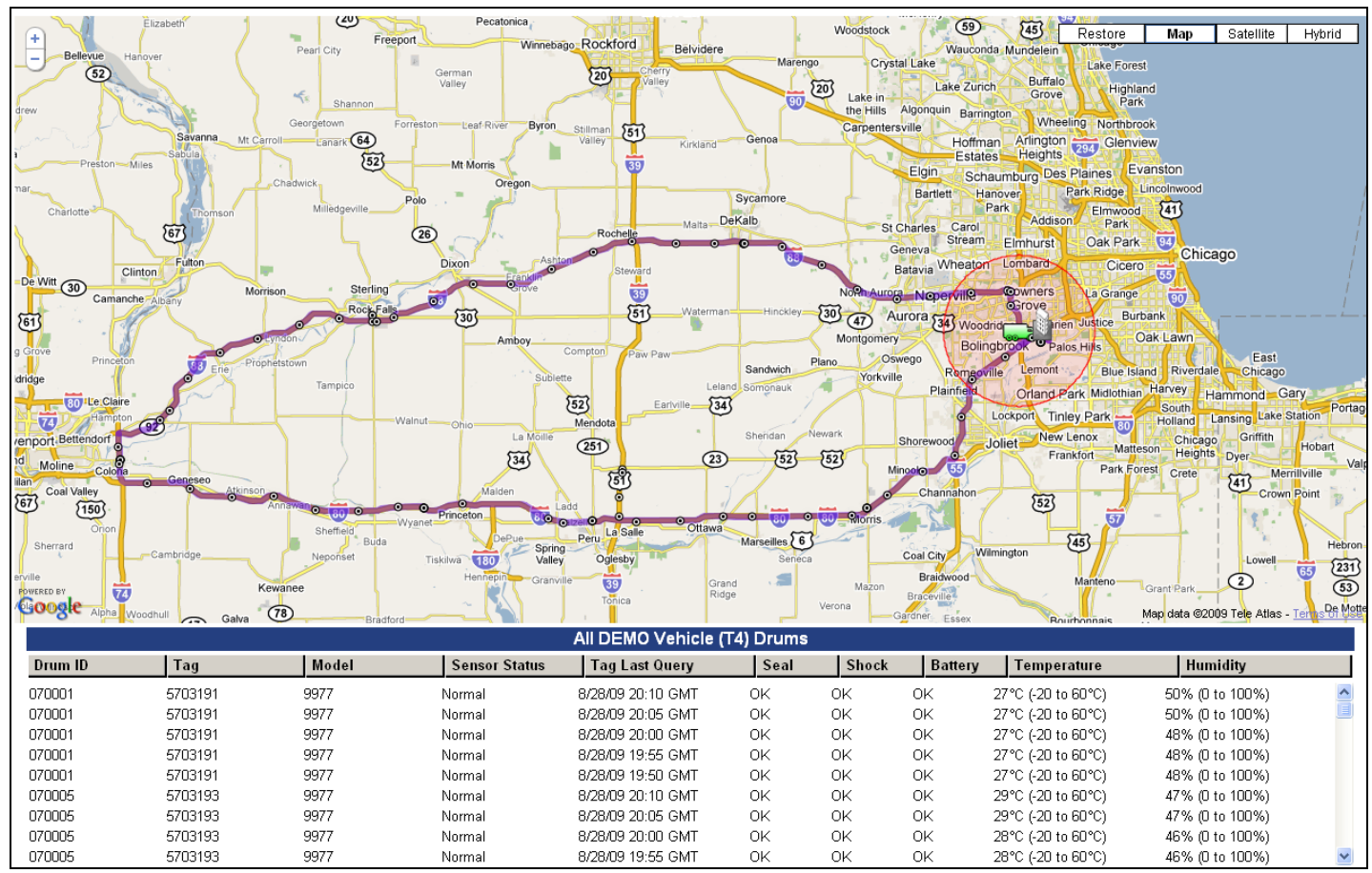


Clicking on the Restore button on the enlarged Google map will reset the map to its original size.

Position the mouse over the Google map and use the scroll wheel, or click on the + or - buttons, to zoom in or out. Emergency and other facilities within a 5-mile radius of the selected vehicle are displayed (as red crosses in the following diagram) if the zoom level is close enough. Click on a facility's icon for more information, including its distance from the current location of the selected vehicle:

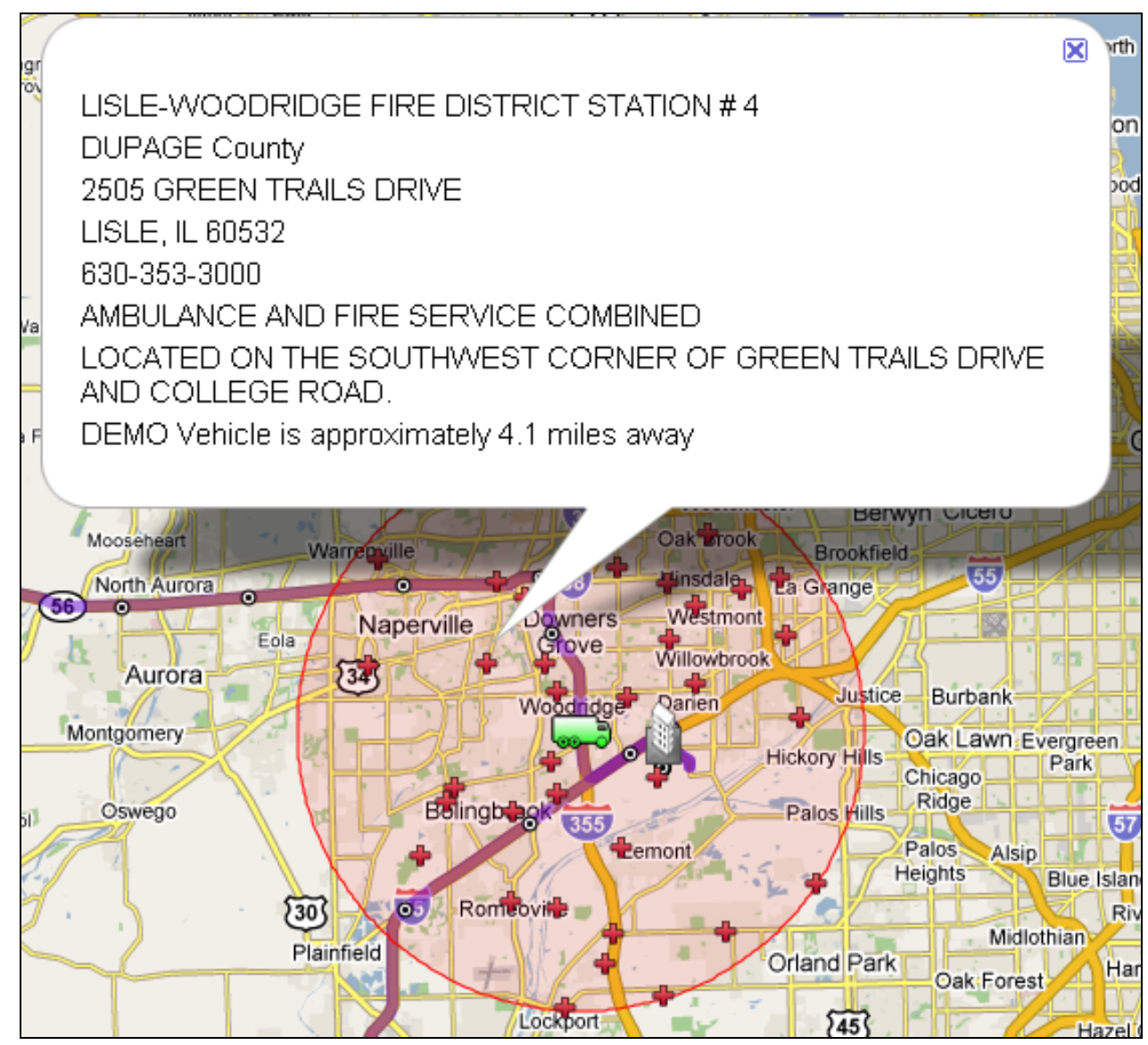

A predetermined transportation route, if defined for the currently selected transportation vehicle, is displayed on the Google map. Along this route are "breadcrumbs" that track the vehicle's actual position. If a cookie crumb (indicated by the symbol $\odot$ ) is clicked, the latitude and longitude of the vehicle at that point in time during the transport will be displayed: 


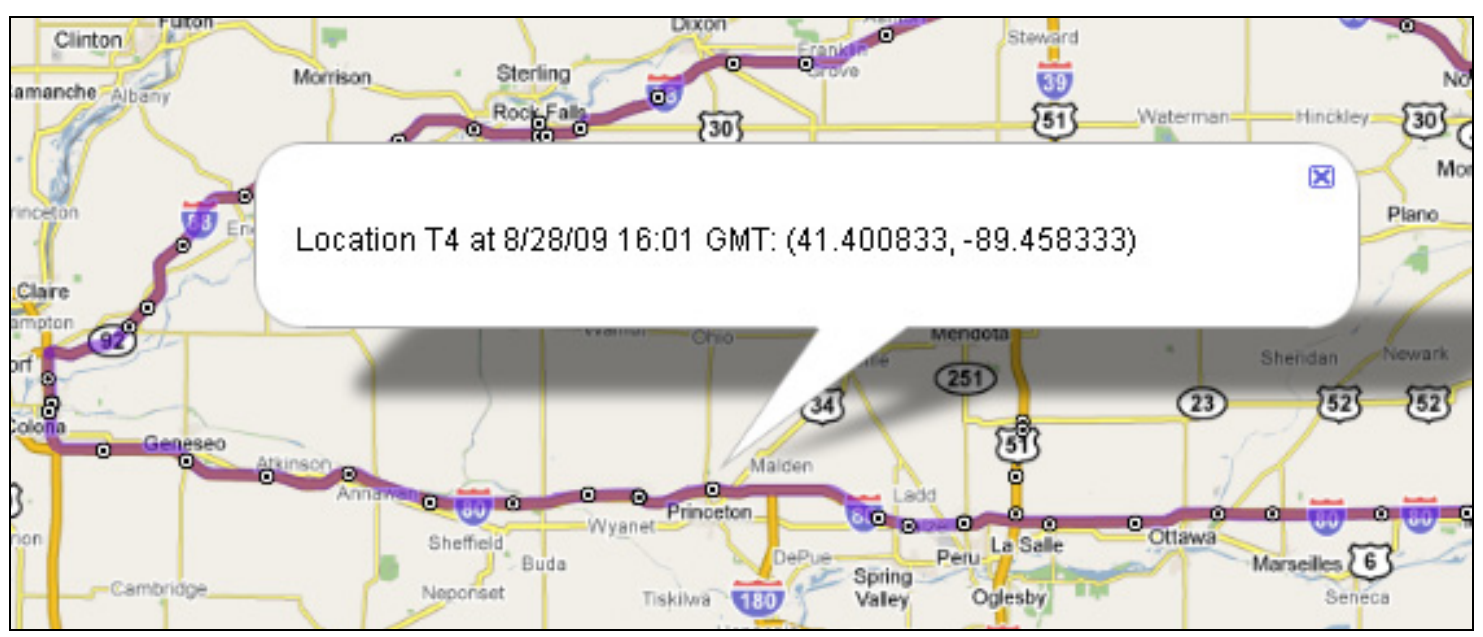

When the U.S. Map button the Google map is clicked, the image changes to a more generic U.S. map:

\section{U.S. Map}

\section{(1)}

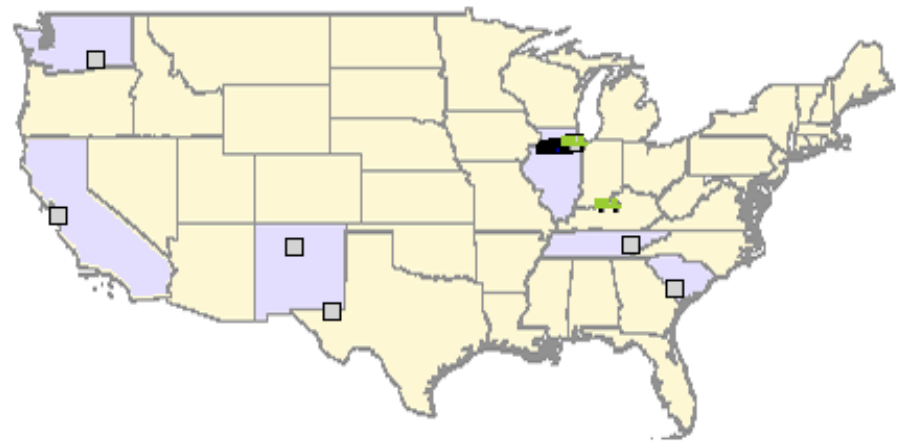

Location: DEMOVehicle $v$ U.S.Map Google Map

Revert to the Google map by clicking the Google Map button.

(2)

The second section of the main web page contains either a site map of a storage location (shown at left, below, for Argonne Building 212), or a diagram of a convoy of vehicles (shown at right, below, for Trucks in DEMO Vehicle). If there is more than one truck in the convoy, users can use the mouse to select a specific vehicle. When the highlighted vehicle changes, other parts of the web page also change accordingly. 


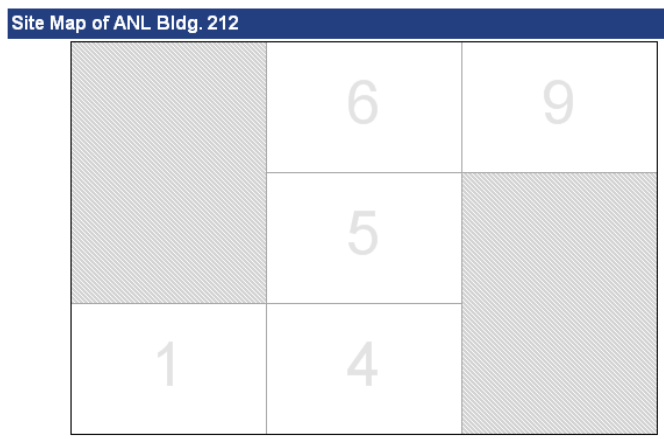

or

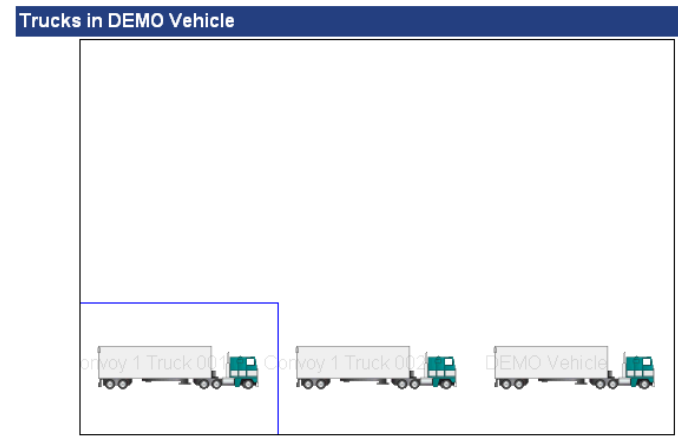

$(3)$

The third section of the main web page contains an overhead view of the selected transportation vehicle shown below or storage zone (not shown). Drums in storage can be stacked up to three high; however, drums in transport are generally single stack on pallets that are tied down securely to the trailer floor. The color of drums indicates normal (green), alarm (red), and warning (yellow), which is not shown. Clicking on a drum (or stack) in this overhead view will select the drum (or stack), as shown by the square enclosing the drum (or stack).

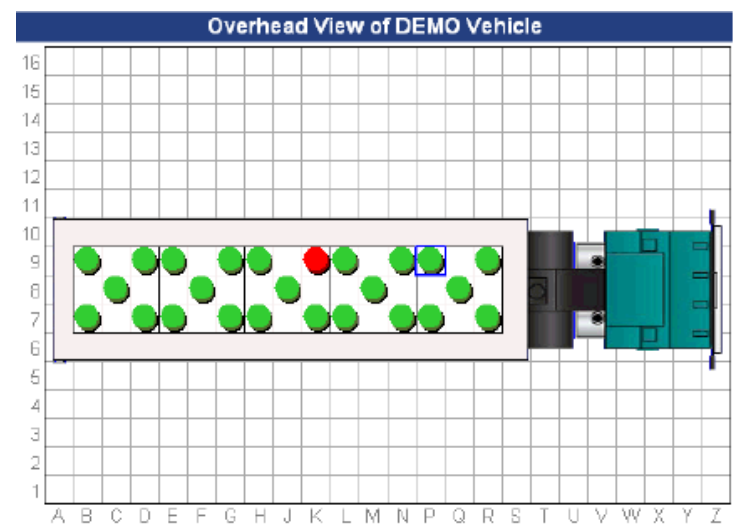

(4)

The fourth section of the main web page contains the current status of the drum selected in section 3 , above. A stack image is preserved for storage application such that any drum shown in the stack image can be selected by clicking the drum with the mouse. 


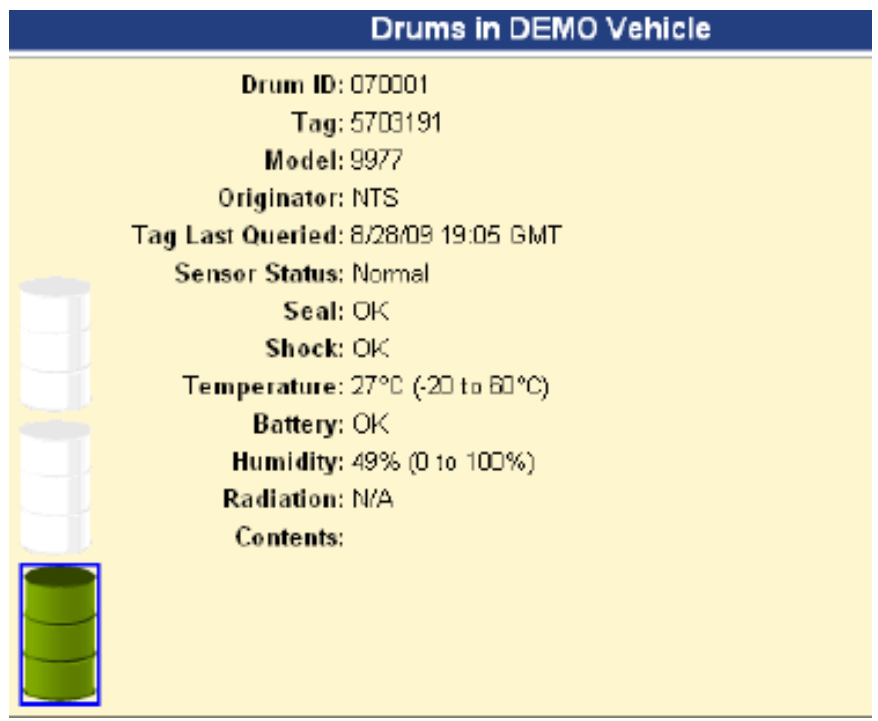

\section{5}

The fifth section of the main web page is a summary status of all drums at either the currently selected storage location or the selected transportation vehicle. By default, this information (and all other information on the web page) is updated only when the Refresh Now button is clicked. The refresh rate of the display (and the entire web page) can be set by selecting a specific time interval from the Refresh Rate drop-down menu:

\begin{tabular}{|l|l|}
\hline Refresh rate: & Manual Refresh Only: \\
\hline Manual Refresh Only \\
\hline 30 seconds \\
1 minute \\
10 minutes \\
30 minutes \\
\hline
\end{tabular}

Refreshing the display reads the information present in the PCP RFID database at that point in time. The current time in GMT (Greenwich Mean Time) is shown at the bottom of the web page.

(6)

The sixth section of the main web page displays the event history of a specific drum. This section changes when a specific drum is selected from the "Overhead View of DEMO Vehicle (3)" or from the "Drums in DEMO Vehicle (4)" section of the web page.

$(7)$

The seventh section of the main web page contains two sets of buttons. The first set is reserved for future implementation of search tools. The second set of buttons is used to create five types of reports, which are displayed as HTML on separate web pages. The reports are described below.

Report 1: Event History of All Drums - This report contains the description and time of all events associated with all drums at all storage locations and in all transportation vehicles. Events are sorted by location, drum, and time. A portion of a sample report is shown below: 


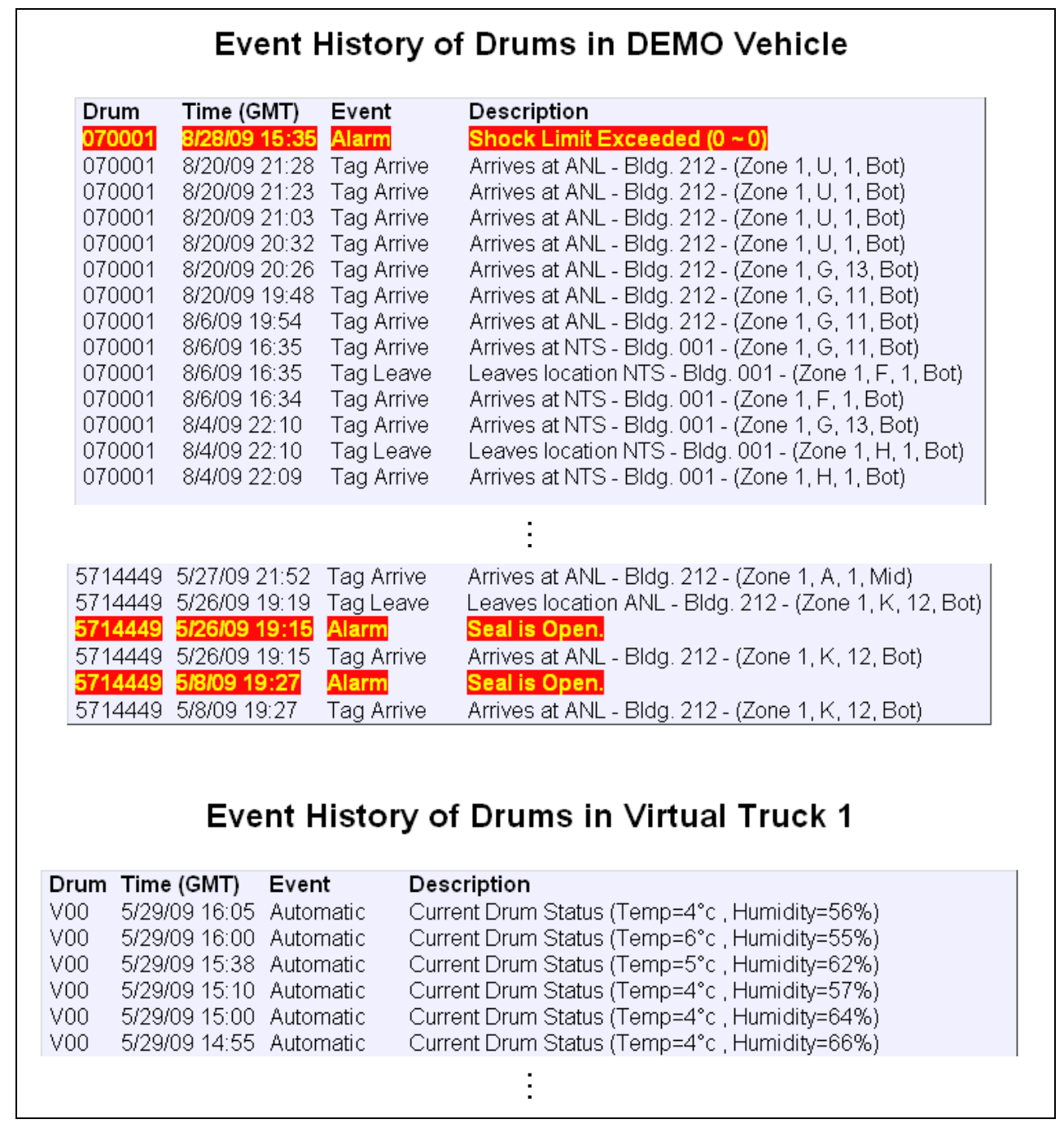

Report 2: Event History at This Location - This report shows the event history of all drums on the currently selected transportation vehicle.

\begin{tabular}{|c|c|c|}
\hline \multicolumn{3}{|c|}{ Event History of Drums in DEMO Vehicle } \\
\hline \multicolumn{3}{|r|}{ History of Drum 070001} \\
\hline Time (GMT) & Event & Description \\
\hline $8 / 2810915: 35$ & Alarm & Shock Limit Exceeded $(0 \sim 0)$ \\
\hline $8 / 20 / 0921: 28$ & Tag Arrive & Arrives at ANL - Bldg. 212 - (Zone 1, U, 1, Bot) \\
\hline $8 / 20 / 0921: 23$ & Tag Arrive & Arrives at ANL - Bldg. 212 - (Zone 1, U, 1, Bot) \\
\hline $8 / 20 / 0921: 03$ & Tag Arrive & Arrives at ANL - Bldg. 212 - (Zone 1, U, 1, Bot) \\
\hline $8 / 20 / 0920: 32$ & Tag Arrive & Arrives at ANL - Bldg. 212 - (Zone 1, U, 1, Bot) \\
\hline $8 / 20 / 0920: 26$ & Tag Arrive & Arrives at ANL - BIdg. 212 - (Zone 1, G, 13, Bot) \\
\hline $8 / 20 / 0919: 48$ & Tag Arrive & Arrives at ANL - Bldg. 212 - (Zone 1, G, 11, Bot) \\
\hline $8 / 6 / 0919: 54$ & Tag Arrive & Arrives at ANL - BIdg. 212 - (Zone 1, G, 11, Bot) \\
\hline $8 / 6 / 0916: 35$ & Tag Arrive & Arrives at NTS - Bldg. 001 - (Zone 1, G, 11, Bot) \\
\hline 8/6/09 16:35 & Tag Leave & Leaves location NTS - Bldg. 001 - (Zone 1, F, 1, Bot) \\
\hline 8/6/09 16:34 & Tag Arrive & Arrives at NTS - Bldg. 001 - (Zone 1, F , 1, Bot) \\
\hline 8/4/09 22:10 & Tag Arrive & Arrives at NTS - BIdg. 001 - (Zone 1, G, 13, Bot) \\
\hline 8/4/09 22:10 & Tag Leave & Leaves location NTS - Bldg. 001 - (Zone 1, H. 1, Bot) \\
\hline $8 / 4 / 0922: 09$ & Tag Arrive & Arrives at NTS - Bldg. 001 - (Zone 1, H, 1, Bot) \\
\hline
\end{tabular}


Report 3: All Information for This Drum - This report shows the sensor and event history for the currently selected drum. A portion of the report is shown below:

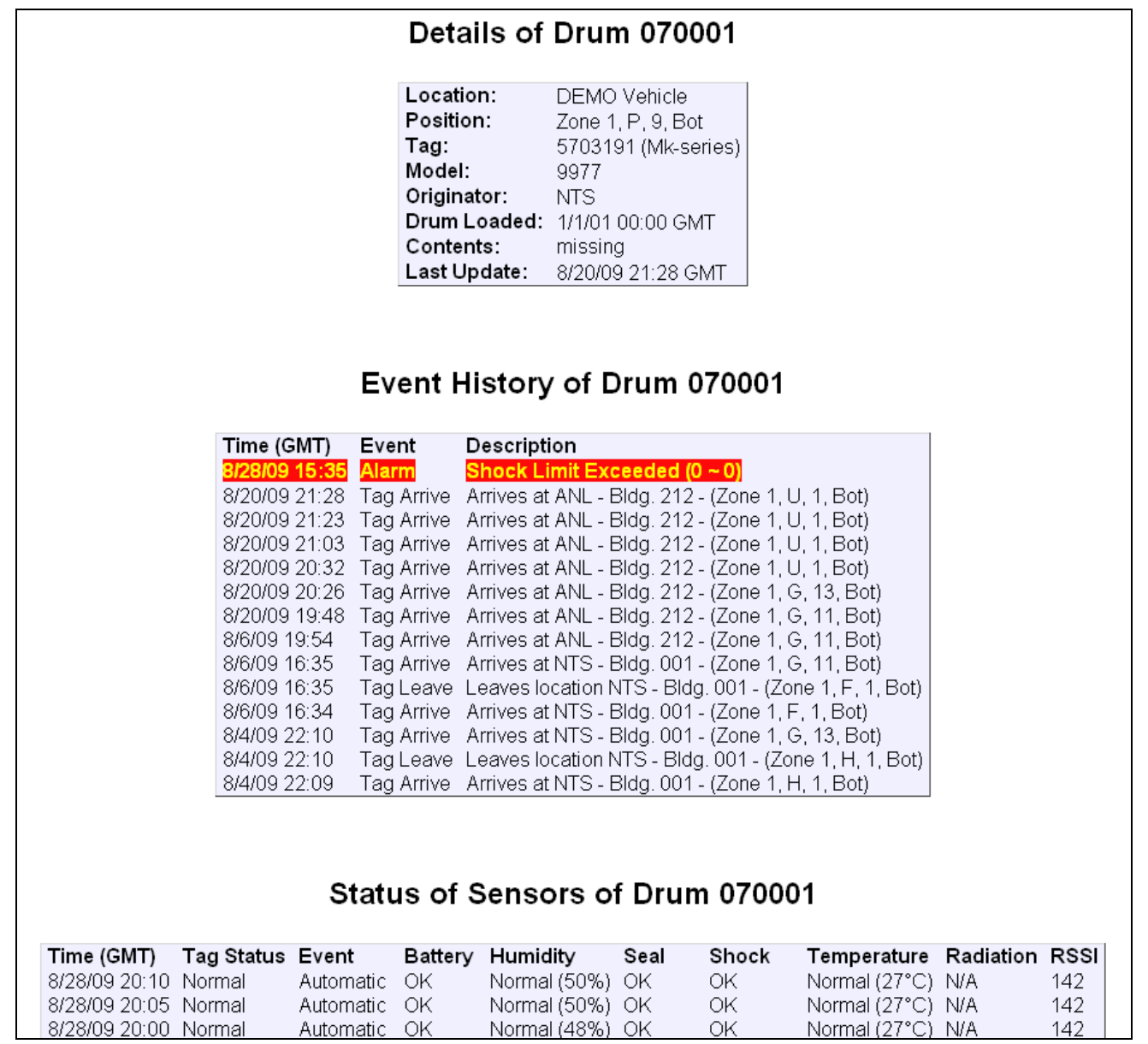

Report 4: History of All Tags during Transport - This report shows the history of all tags that are associated with all drums located on transportation vehicles. Since the complete history of all tags can be thousands of lines, only the first 20 lines are shown on the web page.

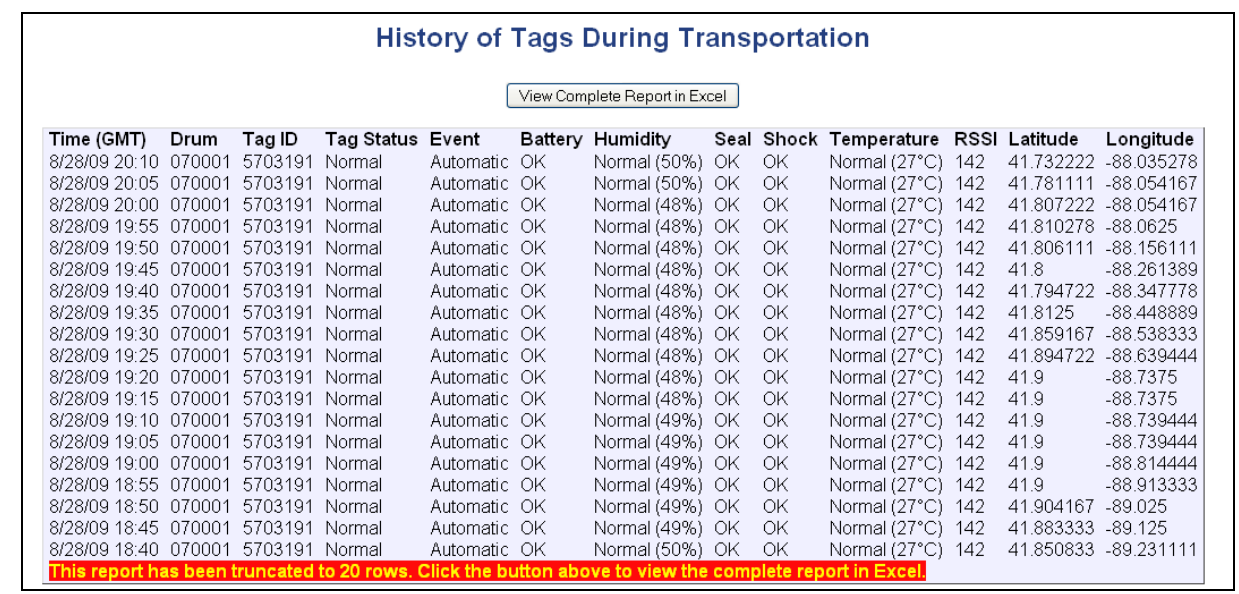


Click the View Complete Report in Excel button to view the entire report. When using MS Internet Explorer, Excel will automatically open and the report will be displayed. If another browser (such as Firefox) is used, right-click on the report, save it as an XML file, and then use Excel to open the XML file. A portion of the Excel file is shown below:

\begin{tabular}{|c|c|c|c|c|c|c|c|c|c|c|c|c|c|c|}
\hline 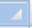 & A & $B$ & c & D & $\mathrm{E}$ & $\mathrm{F}$ & G & $\mathrm{H}$ & 1 & 」 & K & L & M & $\mathrm{N}$ \\
\hline 1 & Time (GMT) & Drum & Tag ID & Tag Status & Seal & Temperature & Humidity & Shock & Battery & RSSI E & Event & Longitude & Latitude & LocationTS \\
\hline 2 & $8 / 28 / 0920: 10$ & 070001 & 5703191 & Normal & OK & Normal $\left(27^{\circ} \mathrm{C}\right)$ & Normal (50\%) & OK & OK & $142 \mathrm{~A}$ & Automatic & -88.03528 & 41.73222 & $8 / 28 / 0920: 11$ \\
\hline 3 & $8 / 28 / 09$ 20:05 & 070001 & 5703191 & Normal & OK & Normal $\left(27^{\circ} \mathrm{C}\right)$ & Normal (50\%) & OK & OK & $142 \mathrm{~A}$ & Automatic & -88.05417 & 41.78111 & 8/28/09 20:06 \\
\hline 4 & $8 / 28 / 0920: 00$ & 070001 & 5703191 & Normal & OK & Normal $\left(27^{\circ} \mathrm{C}\right)$ & Normal (48\%) & OK & OK & $142 \mathrm{~A}$ & Automatic & -88.05417 & 41.80722 & 8/28/09 20:01 \\
\hline 5 & 8/28/09 19:55 & 070001 & 703191 & Normal & OK & ormal $\left(27^{\circ} \mathrm{C}\right)$ & Normal $(48 \%)$ & OK & OK & $142 \mathrm{~A}$ & Automatic & -88.0625 & 41.81028 & 8/28/09 19:56 \\
\hline 6 & $8 / 28 / 0919: 50$ & 070001 & 103191 & Normal & OK & prmal $\left(27^{\circ} \mathrm{C}\right)$ & Normal (48\%) & OK & OK & $142 \mathrm{~A}$ & Automatic & -88.15611 & 1.80611 & 8/28/09 19:51 \\
\hline 7 & $8 / 28 / 0919: 45$ & 070001 & 03191 & Normal & OK & ormal $\left(27^{\circ} \mathrm{C}\right)$ & Normal $(48 \%)$ & OK & OK & $142 \mathrm{~A}$ & Automatic & -88.26139 & 41.8 & $8 / 28 / 09$ 19:46 \\
\hline 8 & $8 / 28 / 0919: 40$ & 070001 & 03191 & Normal & OK & ormal $\left(27^{\circ} \mathrm{C}\right)$ & Normal $(48 \%)$ & OK & OK & $142 \mathrm{~A}$ & Automatic & -88.34778 & 1.79472 & 8/28/09 19:41 \\
\hline 9 & $8 / 28 / 09$ 19:35 & 070001 & 703191 & Normal & OK & Normal $\left(27^{\circ} \mathrm{C}\right)$ & Normal $(48 \%)$ & OK & OK & $142 \mathrm{~A}$ & Automatic & -88.44889 & 41.8125 & 8/28/09 19:36 \\
\hline 10 & 8/28/09 19:30 & 070001 & 703191 & Normal & OK & Normal $\left(27^{\circ} \mathrm{C}\right)$ & Normal $(48 \%)$ & OK & OK & $142 \mathrm{~A}$ & Automatic & -88.53833 & 41.85917 & 8/28/09 19:31 \\
\hline 11 & 8/28/09 19:25 & 070001 & 703191 & Normal & OK & Normal $\left(27^{\circ} \mathrm{C}\right)$ & Normal (48\%) & OK & OK & $142 \mathrm{~A}$ & Automatic & -88.63944 & 41.89472 & $8 / 28 / 0919: 26$ \\
\hline 12 & $8 / 28 / 09$ 19:20 & 070001 & 703191 & Normal & OK & Normal $\left(27^{\circ} \mathrm{C}\right)$ & Normal (48\%) & OK & OK & $142 \mathrm{~A}$ & Automatic & -88.7375 & 41.9 & 8/28/09 19:21 \\
\hline 13 & 28/09 19:15 & 070001 & 91 & Normal & OK & ormal $\left(27^{\circ} \mathrm{C}\right)$ & $(48 \%)$ & OK & OK & $142 \mathrm{~A}$ & Autom & -88.7375 & 41.9 & 8/28/09 19:21 \\
\hline 14 & 8/28/09 19:10 & 070001 & 03191 & Normal & OK & ormal $\left(27^{\circ} \mathrm{C}\right)$ & Normal (49\%) & OK & OK & $142 \mathrm{~A}$ & Autc & -88.73944 & 41.9 & 8/28/09 19:11 \\
\hline 15 & $28 / 09$ 19:05 & 070001 & 03191 & ormal & OK & ormal $\left(27^{\circ} \mathrm{C}\right)$ & Normal $(49 \%)$ & OK & K & $142 \mathrm{~A}$ & Automatic & -88.73944 & 41.9 & 8/28/09 19:06 \\
\hline 16 & /28/09 19:00 & 070001 & 03191 & Normal & OK & ormal $\left(27^{\circ} \mathrm{C}\right)$ & Normal $(49 \%)$ & $\mathrm{OK}$ & OK & $142 \mathrm{~A}$ & Automatic & -88.81444 & 41.9 & 8/28/09 19:01 \\
\hline 17 & /28/09 18:55 & 070001 & 03191 & Normal & OK & ormal $\left(27^{\circ} \mathrm{C}\right)$ & Normal $(49 \%)$ & OK & OK & $142 \mathrm{~A}$ & Automatic & -88.91333 & 41.9 & 8/28/09 18:56 \\
\hline 18 & $8 / 28 / 0918: 50$ & 070001 & 5703191 & Normal & OK & Normal $\left(27^{\circ} \mathrm{C}\right)$ & Normal $(49 \%)$ & OK & OK & $142 \mathrm{~A}$ & Automatic & -89.025 & 41.90417 & 8/28/09 18:51 \\
\hline 19 & $8 / 28 / 09$ 18:45 & 070001 & 5703191 & Normal & OK & Normal $\left(27^{\circ} \mathrm{C}\right)$ & Normal (49\%) & OK & OK & $142 \mathrm{~A}$ & Automatic & -89.125 & 1.88333 & 8/28/09 18:46 \\
\hline 20 & 8/28/09 18:40 & 070001 & 03191 & Normal & OK & Normal $\left(27^{\circ} \mathrm{C}\right)$ & Normal $(50 \%)$ & OK & OK & $142 \mathrm{~A}$ & Automatic & -89.23111 & 41.85083 & $8 / 28 / 09$ 18:41 \\
\hline 21 & 8/28/09 18:35 & 070001 & 91 & Normal & OK & Normal $\left(27^{\circ} \mathrm{C}\right)$ & $(50 \%)$ & OK & OK & $142 \mathrm{~A}$ & Autom & -89.33639 & 41.82278 & 8/28/09 18:36 \\
\hline 22 & 8/28/09 18:30 & 070001 & 03191 & Normal & OK & ormal $\left(27^{\circ} \mathrm{C}\right)$ & Normal (50\%) & OK & OK & $142 \mathrm{~A}$ & Automatic & -89.43222 & 41.82278 & 8/28/09 18:31 \\
\hline 23 & $8 / 28 / 0918: 25$ & 070001 & 03191 & ormal & OK & ormal $\left(27^{\circ} \mathrm{C}\right)$ & Normal (50\%) & OK & OK & $142 \mathrm{~A}$ & Automatic & -89.53222 & 41.78944 & 8/28/09 18:26 \\
\hline 24 & $28 / 0918: 20$ & 070001 & 03191 & Normal & OK & ormal $\left(27^{\circ} \mathrm{C}\right)$ & Normal $(50 \%)$ & OK & OK & $142 \mathrm{~A}$ & Automatic & -89.63528 & 1.75917 & 8/28/09 18:21 \\
\hline 25 & 28/09 18:15 & 070001 & 191 & Normal & OK & ormal $\left(27^{\circ} \mathrm{C}\right)$ & Normal $(50 \%)$ & OK & OK & $142 \mathrm{~A}$ & Automatic & -89.67806 & 75083 & 8/28/09 18:18 \\
\hline 26 & $28 / 0918: 10$ & 070001 & 5703191 & Normal & OK & Normal $\left(27^{\circ} \mathrm{C}\right)$ & Normal $(50 \%)$ & OK & OK & $142 \mathrm{~A}$ & Automatic & -89.68944 & 41.7625 & 8/28/09 18:11 \\
\hline 27 & 8/28/09 18:05 & 070001 & 703191 & Normal & OK & Normal $\left(27^{\circ} \mathrm{C}\right)$ & Normal (50\%) & OK & OK & $142 \mathrm{~A}$ & Automatic & -89.68944 & 41.75083 & 8/28/09 18:06 \\
\hline 28 & $8 / 28 / 0918: 00$ & 070001 & 5703191 & Normal & OK & Normal $\left(27^{\circ} \mathrm{C}\right)$ & Normal $(50 \%)$ & OK & OK & $142 \mathrm{~A}$ & Automatic & -89.775 & 41.75722 & 8/28/09 18:01 \\
\hline 29 & $8 / 28 / 09$ 17:55 & 070001 & 3191 & Normal & OK & Normal $\left(27^{\circ} \mathrm{C}\right)$ & Normal $(50 \%)$ & OK & OK & $142 \mathrm{~A}$ & Automatic & -89.87694 & 1.74472 & 8/28/09 17:56 \\
\hline 30 & $8 / 28 / 09$ 17:50 & 070001 & 91 & Norn & OK & ormal $\left(23^{\circ} \mathrm{C}\right)$ & Norm & OK & OK & $142 \mathrm{~A}$ & atic & -89.98333 & 1861 & 8/28/09 17:51 \\
\hline 31 & $8 / 28 / 09$ 17:45 & 070001 & 03191 & Normal & OK & ormal $\left(23^{\circ} \mathrm{C}\right)$ & Normal (48\%) & OK & 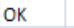 & $142 \mathrm{~A}$ & atic & -90.08 & 9056 & 8/28/09 17:46 \\
\hline 32 & $8 / 28 / 0917: 40$ & 070001 & 03191 & Normal & OK & Normal $\left(23^{\circ} \mathrm{C}\right)$ & Normal $(48 \%)$ & OK & UK & $142 \mathrm{~A}$ & Automatic & -90.16333 & 41.6425 & $8 / 28 / 09$ 17:41 \\
\hline 33 & 8/28/09 17:35 & 070001 & 03191 & Normal & OK & Normal $\left(23^{\circ} \mathrm{C}\right)$ & Normal (48\%) & OK & OK & $142 \mathrm{~A}$ & Automatic & -90.20917 & 41.58111 & 8/28/09 17:36 \\
\hline 34 & 8/28/09 17:30 & 070001 & 5703191 & Normal & OK & Normal $\left(23^{\circ} \mathrm{C}\right)$ & Normal (48\%) & OK & OK & $142 \mathrm{~A}$ & atic & -90.23417 & 41.5625 & 8/28/09 17:35 \\
\hline 35 & $28 / 09$ 17:25 & 070001 & 5703191 & Normal & OK & Normal $\left(23^{\circ} \mathrm{C}\right)$ & Normal $(48 \%)$ & OK & OK & $142 \mathrm{~A}$ & Automatic & -90.34056 & 1.51139 & 8/28/09 17:28 \\
\hline 36 & $28 / 09$ 17:20 & 070001 & 5703191 & al & OK & Normal $\left(23^{\circ} \mathrm{C}\right)$ & $\operatorname{rmal}(48 \%)$ & OK & OK & 142 & tomatic & -90.33417 & 8639 & $8 / 28 / 09$ \\
\hline 37 & 9 17:15 & 0 & & 1 & OK & Normal $\left(23^{\circ} \mathrm{C}\right)$ & 48\%) & OK & $\mathrm{OP}$ & A & tomatic & -90.33417 & 1.48639 & 8/28/09 17:16 \\
\hline 38 & $8 / 28 / 09$ 17:11 & 070001 & 5703191 & Normal & $\mathrm{OK}$ & Normal $\left(23^{\circ} \mathrm{C}\right)$ & Normal (48\%) & OK & OK & $142 \mathrm{~A}$ & Automatic & -90.33417 & 41.48639 & 8/28/09 17:12 \\
\hline
\end{tabular}

Report 5: GIS Report - When a transportation vehicle is selected and this button is clicked, a report is generated that contains a map showing the current location of the vehicle, a 5-mile buffer zone around the vehicle, and various facilities within that zone. At this time, only emergency medical facilities are displayed. In addition to the map, a table is created that lists each facility, relevant contact information, and its approximate distance to the selected vehicle. A portion of this report is shown below; complete GIS reports generated during the MiniDemo can be found in Appendix D. 


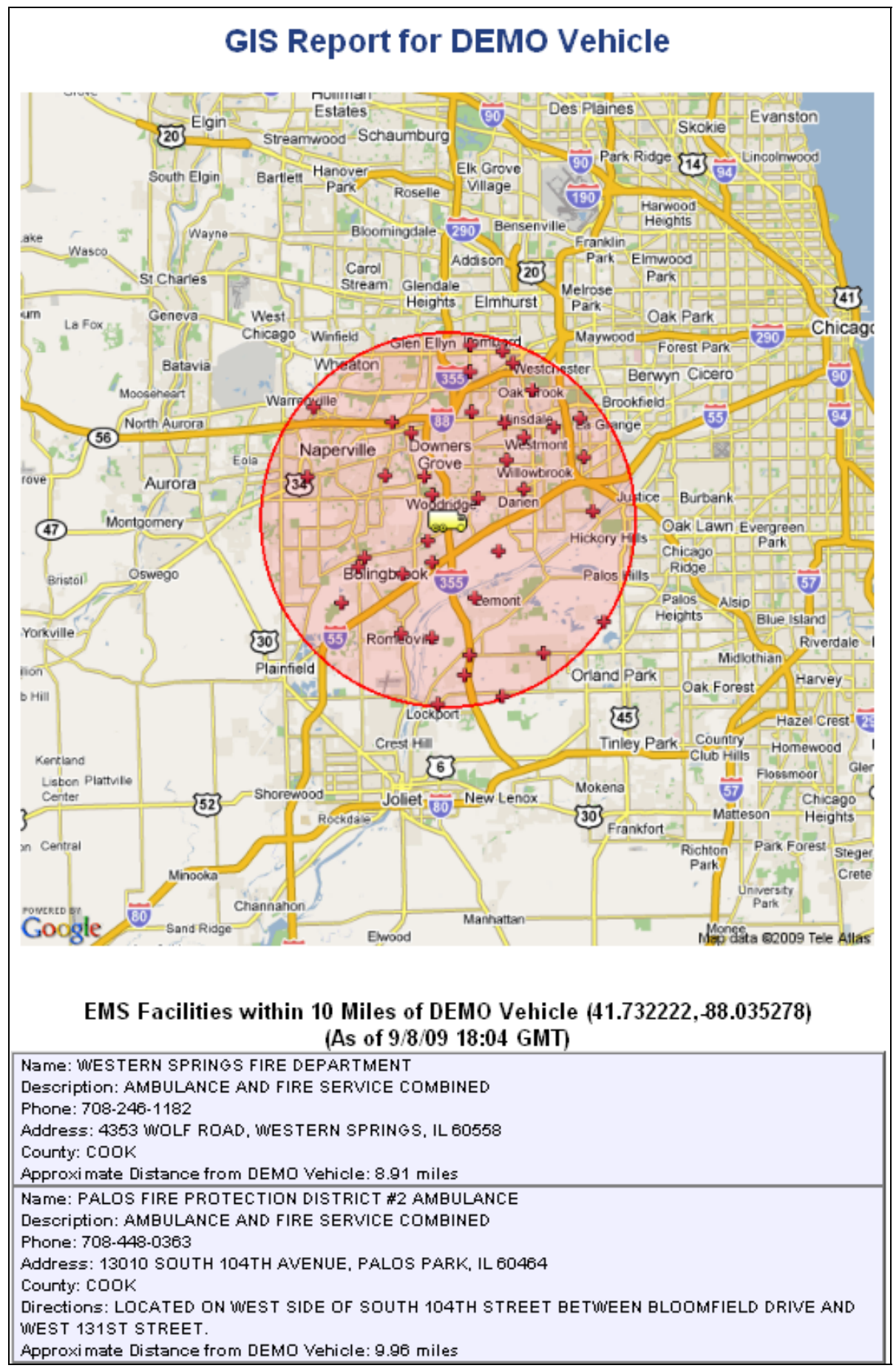




\section{System Performance}

\subsection{Route Tracking and Web Functions}

The MiniDemo used integrated hardware and software sets from Qualcomm (OmniTRACS) and Argonne (ARG-US) in an algorithm that had not been tried and tested before. All data from the MiniDemo indicated that the integration was successful and that the system worked as designed. The performance of enhanced features for tracking — planned route, route tracking (bread-crumbing), zooming, spot information, and detailed tag information display — was verified in the MiniDemo.

The preplanned route of the MiniDemo is shown in Figure 5. The vehicle started the trip at Argonne National Laboratory; went to East Moline, IL, via I-80; and returned to Argonne via I-88, for a total distance of $\approx 300$ miles in slightly less than 6.5 hours. Six staged incidents were planned and conducted at the numbered locations shown in Figure 5.

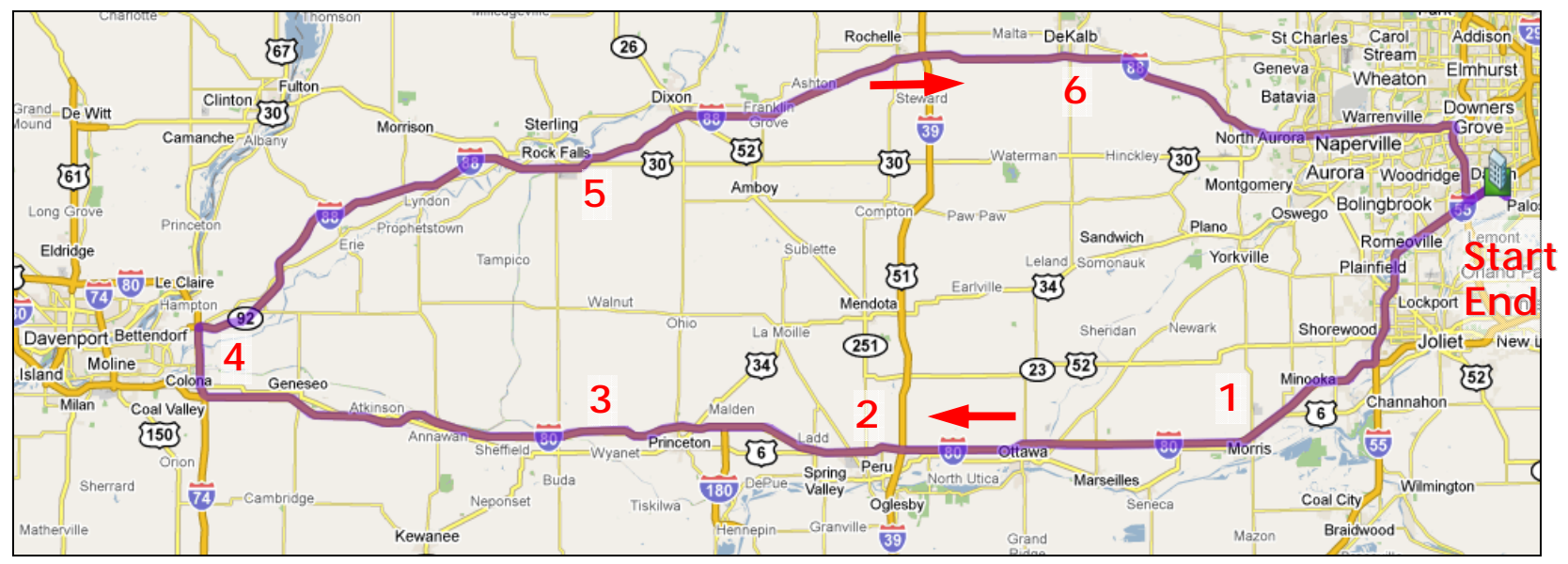

FIGURE 5 Planned route for the MiniDemo and the locations for the six staged incidents.

At the preset 5-minute intervals, the OmniTRACS transponder acquired the latitude and longitude information of the vehicle from the U.S. GPS satellites and sent the information, along with the RFID tag data, to the Qualcomm hub. For each of the staged incidents, the latitude/longitude data and the event information were sent to the hub immediately. The route traveled by the vehicle is visible as breadcrumbs, as shown in Figure 6, on the tracking webpage. Multiple breadcrumbs at a location, typically just off the planned route, are an indication that the vehicle was stopped for staged incidents or acquiring supplies. Along Route IL-51 (near the bottom center of Figure 6), there was a short, unintentional detour. This detour was accurately captured by the tracking system. Such features may be further refined for "route deviation" detection functions (i.e., route geofencing). 


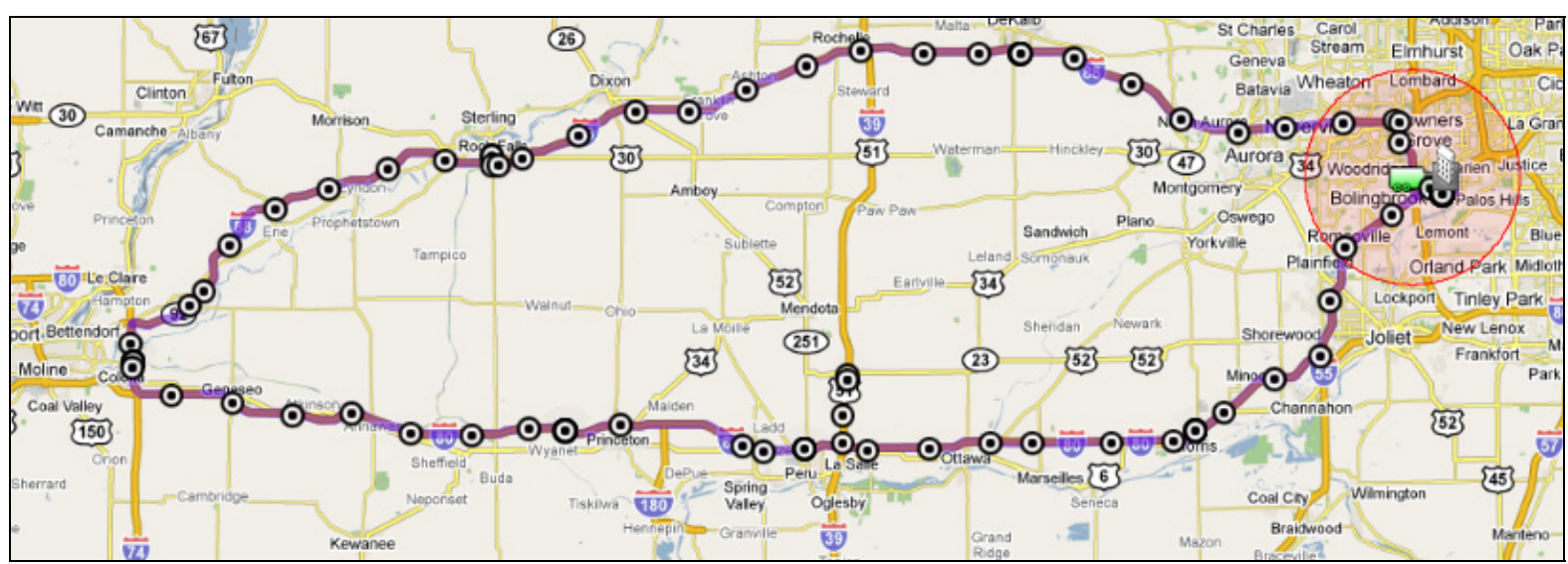

FIGURE 6 Actual route traveled ("bread-crumbing") in the MiniDemo.

At anytime during the transport, detailed or abbreviated information of the "tagged drums" in the vehicle can be viewed on the webpage, as illustrated in a screen capture in Figure 7. The map may be superimposed with satellite or terrain information, if desired.

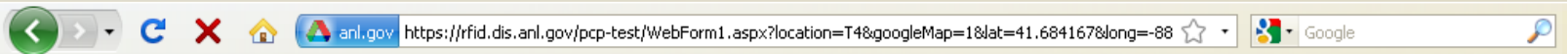
[.] Most Wisited $\boldsymbol{E}$ Customize Links $\boldsymbol{\pi}$ Free Hotmail $\square$ Windows Marketplace $\square$ Windows Media $\square$ windows

*: Packagings RFID Tracking System

E Environutatal Management safety \& performance " cleanup a closure

\section{Packagings RFID Tracking System}
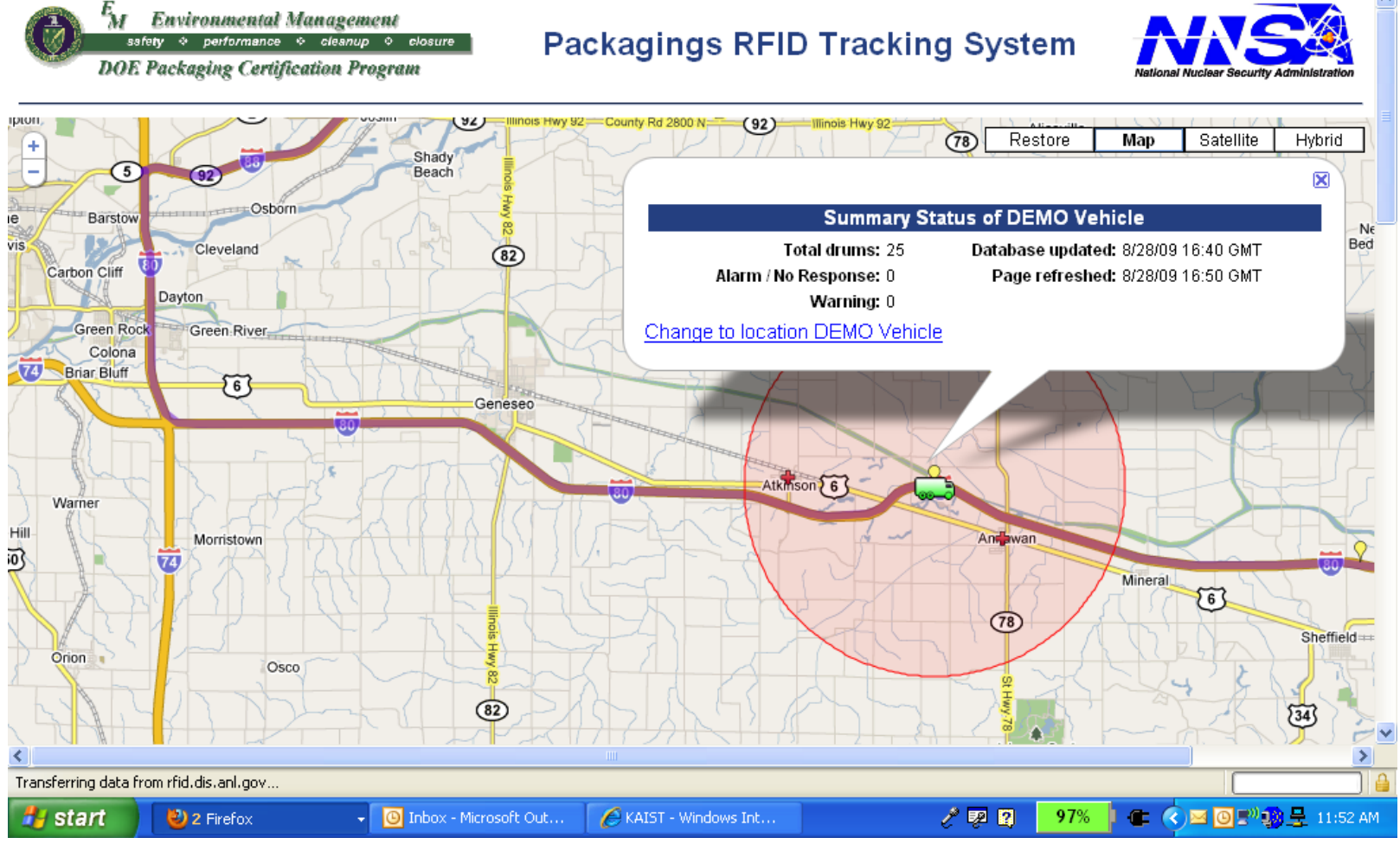

FIGURE 7 Summary status of tags, as captured by a screenshot. 


\subsection{Tag Performance}

Twenty-five (25) Mk-I and -II RFID tags were used in the MiniDemo. As stated before, the tags were not mounted on drums because of the lack of a full complement of such drums at Argonne. However, from the standpoint of tag performance and satellite communication, whether the tags are mounted or not did not materially affect the test results. The seal integrity sensors of the tags were compressed during the MiniDemo, simulating the mounted state of tags on drums, except during three of the staged incidents.

All 25 tags reliably communicated with the reader during the MiniDemo. There were no malfunctions of any tags. Sensor readings were accurate the entire time. (See Appendix C for the recorded data of the tags during the entire MiniDemo.)

Six tags, randomly selected from the lot, were used in staged incidents - three simulated seal-sensor violation (i.e., open) and three simulated excessive shock (by hand tapping). All six tags performed as expected and generated the required alarm signals. The three seal-sensor alarms were successfully cleared after the seal bolts were retightened.

\subsection{Communication Reliability}

The equipment in the MiniDemo vehicle performed as desired. Messages that contained information on the status of the tag were sent to the OmniTRACS transponder every five minutes, but the messages related to the staged incidents were sent immediately. Because of the satellite bandwidth, the transponder required approximately 1.8 minutes to send a full message containing information on 25 tags in the vehicle to the satellite. The time required for sending a message varied slightly, depending on the length of the message and the status of the satellite communication.

During the MiniDemo, occasionally the satellite service was not available, possibly because line- of-sight to the data satellite was temporarily lost (due to blockage from buildings, overpasses, etc.) The off time was typically brief (i.e., less than a minute). When that happened, the messages stayed in an output queue in the transponder and were sent when satellite communication was restored. The same method was used to handle interruptions caused by staged incidents, which generated alarm messages that took precedence over the normal data messages. Operating in this manner, no messages were lost throughout the MiniDemo, except one: this message (at 15:55 GMT) could not be found in the Qualcomm hub, although it was preserved in the control computer. The cause for this missing data line is being investigated. One possible cause could be a momentary disturbance of the serial connection between the RFID control computer and the tablet.

All of the messages pulled off the Qualcomm Hub contain additional intermediate Qualcomm time stamps, such as for "event" and "position," in addition to the final "received" time stamp. The overall time lags between RFID event time and Qualcomm "received" time is shown in Figure 8. This lag includes the $\approx 1.8$ minutes (shorter for staged incident messages) required for completing the satellite transmission and internal processing at the Qualcomm Hub, plus any delay due to the lack of satellite availability. For the majority of data, the lag is between 2 and 7 minutes - a range that appears consistent with the 4-7-minute intervals for position and messaging noted for the DOE TRASNCOM system (TRANSCOM 2009). 


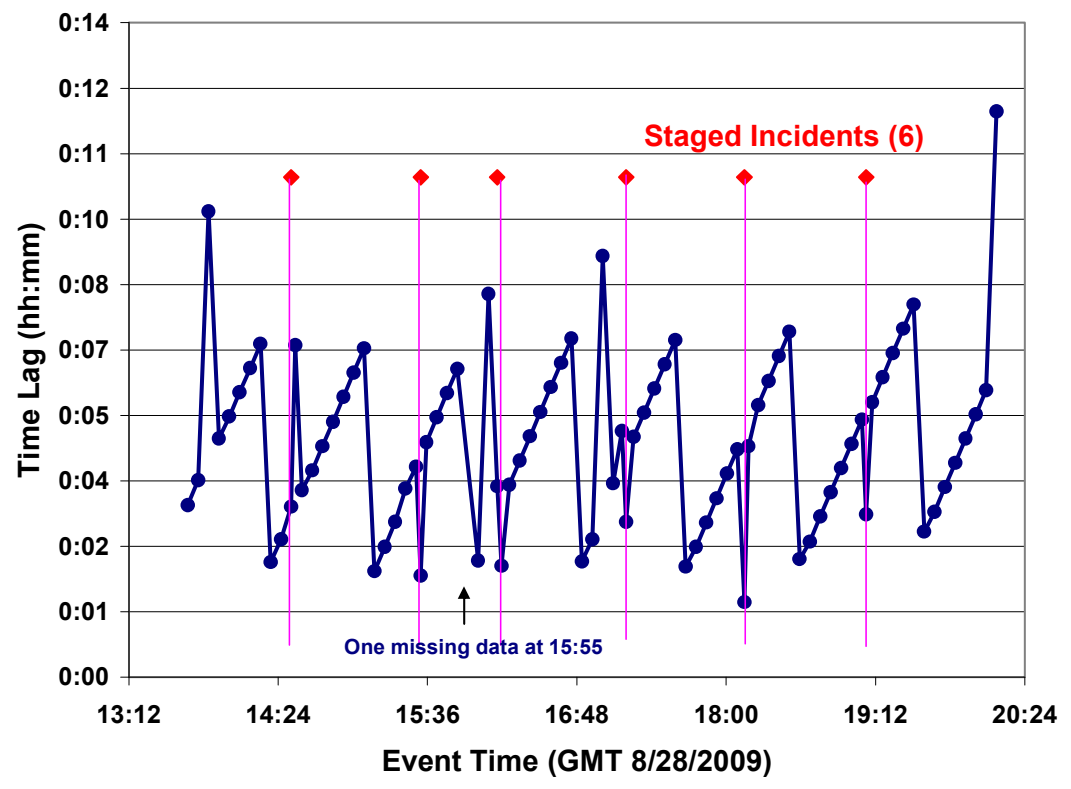

FIGURE 8 Time lags between sent (from vehicle) and received (at Qualcomm Hub). Lags for staged incidents were typically smaller, as the message is brief and takes precedence in the send priority.

\subsection{System Performance - Staged Incidents}

There were six staged incidents during the transportation segments of the demo. The time, location, offending tag (drum), and nature of these incidents (i.e., seal or shock sensor violations) are shown Appendix $\mathrm{C}$ and Figure 5. The three seal violations were produced by loosening the drum bolts, and the three shock violations were produced by a hand tap. When the field team created a staged incident, the reader and ARG-US software detected the alarm and promptly sent it to the OmniTRACS transponder. The transponder then transmitted the alarm message to the Qualcomm hub via the satellite without delay (i.e., interrupting whatever operation the transponder was doing at the time). At 1-minute intervals, the Argonne Command Center polled and retrieved the new message from the Qualcomm hub, processed the data, and posted the alarm on the web page. Concurrently, the designated recipients were sent a concise notification of the incident, by e-mail, text message, or both.

For the staged incidents, the cause of the incident was known and the alarm was cleared by the field personnel shortly after notifying the Command Center. In three of the staged incidents, the field team additionally requested that mockup GIS spatial query reports be issued by the Command Center. Such reports, shown in Appendix D, were compiled quickly on the basis of the existing geodatabases on the server and were issued within minutes. Two of the reports were for buffer zones of 5-mile radius and one for a buffer zone of 10-mile radius. The size of the buffer zone and the contents of the GIS reports can be adjusted and tailor-made on the basis of the circumstances and situations. 


\section{Discussion}

\subsection{Integration of RFID and Qualcomm Components}

The most important purpose of this MiniDemo is to verify whether there are incompatibilities between the PCP RFID components and the Qualcomm's OmniTRACS communication equipment. This is the prerequisite step for incorporating the RFID item-tracking and monitoring features with any of the professional vehicle tracking systems in the field, such as DOE TRANSCOM, which presently uses the Qualcomm's OmniTRACS.

In this regard, the MiniDemo is a success. Using the available serial port on the Qualcomm OmniTRACS tablet, it was demonstrated that carefully structured RFID data can be successfully transmitted via the satellite and received at the Qualcomm hub. Pulling the received data off the Qualcomm hub into Argonne's secure sever was also accomplished with no difficulties.

\subsection{Need for Enhanced RFID Reader Performance}

The reader in the RFID system currently collects the raw data from the tags and passes it on to the data processor (served by a laptop computer), which, in turn, processes it into suitable packets for satellite transmission. It is imperative that the reader's functionality be enhanced to handle the data processing duty so that the stand-alone data processor could be eliminated. This would reduce the clutter in the cab, as well as the workload of the vehicle operator.

A customized reader with a built-in CPU can most likely serve the function. A discussion is under way with an RFID component vendor for such a development.

\subsection{Need for Two-Way Communication between Reader in Truck and Command Center}

In the MiniDemo, the flow of the RFID data was one-way: from the reader, via the Qualcomm hub, to the Command Center. While item tracking and monitoring can be satisfactorily performed in this manner, operational flexibility is constrained - the truck driver will have to undertake all of the hands-on actions. Such flexibility, including the ability by the Command Center to reset a tag alarm, or actively poll the status of a given tag, would be highly desirable in transport implementations. Two-way communication is already in place in commercial vehicle tracking, such as the DOE TRANSOM/Qualcomm system, including the ability to even disable a vehicle remotely from the Command Center.

To implement two-way RFID communication, at least two parts of development work are needed. The first part is to send a command message from the Command Center, via web link to the Qualcomm hub. The second part is for the ARG-US software to receive the command message, in Free-Form Text format, from the OmniTRACS transponder and execute the command. The development is technically feasible and can be completed with a modest effort. 


\subsection{Need for High-Frequency In-Vehicle Polling}

In the MiniDemo, the tag polling frequency was set at every 5 minutes. The satellite transmission frequency was also set at every 5 minutes (i.e., after each poll).

While polling the tag at this rate is more than adequate for gathering most data from tag sensors, such as temperature, it may not be adequate in some cases. For instance, if a drum is diverted, it may take as long as 5 minutes for the reader to sense that the tag/drum is missing. Likewise, if a tag is malfunctioning or the battery is exhausted, it would also not be detected until the next scheduled poll. For route-deviation tracking, or geofencing, higher-density vehicle position data may be necessary.

It is possible to poll the drums in the truck continuously. The rate depends on the time required to complete a polling cycle of the 25 tagged drums in the truck - up to $\approx 10$ seconds. The tag batteries can handle this extra workload - it has been estimated that a single battery can last more than 10 days under continuous polling conditions, and a tag has four such batteries.

With high-frequency polling, when the conditions of all drums are normal, the interval for satellite transmission could still be 5 minutes, and only the last data set would be sent. (Sending data more frequently would be uneconomical and likely unnecessary.) When an abnormal event is encountered, the trigger information would be sent immediately, just as in this MiniDemo. Route-deviation tracking would be conducted in a similar manner - vehicle position data would be compared continuously with that of the pre-planned route, and any deviation would be reported immediately.

\subsection{Further Enhancing Web Page Functionality}

While the web page functionality is vastly improved in this MiniDemo over that used in the April 2008 DEMO, the following areas have been identified as possible future enhancements:

- Add the ability to zoom into the Google map to view data (such as "breadcrumbs") at a higher resolution. This reduces the amount of clutter seen on the map when it is zoomed out.

- Support for ESRI (Environmental Systems Research Institute) shapefiles could be added to simplify the display of transportation routes and icons on the Google map. This enhancement will simplify and standardize the data format used by the web page's interface, since the structure of the database can be modified to accommodate the transportation route displays.

- Database code could be re-factored to speed up the response time of the web page. This improvement could be critical as the number of tag status records grows. Changes to the database code will simplify the development of additional features that may be useful as the system matures.

- It may be appropriate to create two versions of the web site: one that emphasizes the static layout of storage locations and one that emphasizes dynamic mapping and GIS features for transportation vehicles. A web page that shows static storage locations would have features (such as live video surveillance images, pallet and drum positions, and drum stacking) that are not essential for displaying transportation vehicles. Conversely, there are features of a web page showing dynamic 
transportation vehicles that are not relevant when static storage locations (such as planned routes, breadcrumbs, and route-deviation alerts, etc.) are viewed.

- The web page should be updated to facilitate two-way communication, as discussed before. For instance, an administrative web site could be developed to allow authorized users to clear alarms and request query updates. A condensed display can also be designed to let an administrator view the status of all drums at all sites at a "single-glance." This condensed display of summary status can run in the background on the tablet display for viewing by the vehicle operator.

- The web page should have streamlined processes to move drums between locations and to initiate new trips for transportation vehicles.

- The web page should be enhanced to allow multiple transportation vehicles to be viewed on multiple windows/monitors. The amount of pixels needed to display the current web page on a computer monitor is larger than the actual monitor's dimensions, thus necessitating cumbersome scrolling to view all the information. Adding the ability to display the page on more than one monitor would let the operator view more information without having to scroll the window or open new web pages.

- Emergency medical service locations are shown on the Google map when a transportation vehicle is selected. Other types of location data, such as schools, hospitals, police stations, stadiums, laboratories, truck stops, tunnels, hotels or motels, could be added to the database for display on the Google map and/or in the GIS report. 


\section{Conclusions}

The feasibility of integrating Qualcomm's OmniTRACS equipment and infrastructure with the PCP RFID system was demonstrated, judging from the ability to successfully conduct the following tasks:

- Polling the RFID tags in the vehicle,

- Transmitting the data via satellite to the Qualcomm Hub with the added timing and vehicle position information,

- Retrieving the data from the Qualcomm Hub, and

- Processing and displaying the information on a secure webpage.

The MiniDemo showed that the system can competently handle both normal and off-normal conditions (incidents) during transportation. In the case of off-normal conditions, alarm messages can be sent promptly to the Command Center for action. Spatial query reports, based on a geographic information system (GIS), can be quickly prepared for the first responders in case of a transport incident. The ability to issue such reports at a moment's notice has been built into the new RFID web functions.

To attain further operational flexibility, the home-base Command Center should be empowered to have full control of the transport operation. To achieve this end, two-way communication with the on-vehicle RFID system needs to be implemented. The algorithm should be straightforward, and only a moderate effort is anticipated before actual demonstration. The RFID two-way communication is an add-on to the existing control system for the vehicle.

The information gained from the MiniDemo is valuable for the full implementation of RFID tracking and monitoring of nuclear materials containers during transportation. A recommended path forward is to integrate the PCP RFID system with a matured vehicle tracking system, such as the DOE TRANSCOM, that is being used routinely for radioactive materials and waste shipments. A joint development project is being planned between the PCP and the DOE TRANSCOM in the near future. Adding an RFID interface to TRANSCOM is a significant enhancement to the DOE infrastructure for tracking and monitoring "high-visibility" shipments of radioactive materials. 


\section{References}

Chen, K., et al., 2009, "ARG-US - An RFID-Based Tracking and Monitoring System for Nuclear Material Packages," Proc. 50 ${ }^{\text {th }}$ INMM Conf., Tucson, AZ, July 12-16.

QUALCOMM Incorporated, 2004, “OmniTRACS and OmniExpress Mobile Interface Protocol Technical Reference,” 80-52461-1 Rev. J, October.

TRANSCOM, 2009, “TRANSCOM Fact Sheet,” DOE EM Transportation, April.

Tsai, H.C., et al., 2008, "Report on Demonstration (DEMO) of Radio Frequency Identification (RFID) Tracking System," conducted for the DOE Packaging Certification Program of U.S. Department of Energy Environmental Management, Office of Packaging and Transportation, Washington, D.C., September 30. 


\title{
Appendix A
}

Example 1: A Free-Form Text (FFT) Message

\begin{abstract}
$091020092005295714000 \quad 0007701602800080 B 05714001$ $0003 C 016028000813057140020002 C 01602800081105714003$ 0004B01602800081305714004 0000901602800081005714005 $0000501602800080 B 057140060005001602800080 C 05714007$ 0000601602800081205714008 0000A01602800081105714009 $0003501602800080 C 057140100004501602800081105714011$ 0005201602800080B05714012 0003101602800080E05714013 0004801602800081305714014 0001E01602800080E05714015 $0003 C 01602800081005714016 \quad 0004 A 01602800081305714017$ $00043016028000810057140180002701602800080 F 05714019$ $0007501602800080 E 05714020 \quad 0004001602800080 C 05714021$ 0002501602800081305714022 O006E01602800080B05714023 OOOOF01602800080A05714024 0007101602800080F0
\end{abstract}

Note: The above message contains the data for 25 RFID tags preceded by a time stamp.

Example 2: A Byte Array (shown in Hex) Sent Over-the-Air

C000070000000000303039313032303039323030353239353731343030302020203030303737 3031363032383030303830423035373134303031202020303030334330313630323830303038 3133303537313430303220202030303032433031363032383030303831313035373134303033 2020203030303442303136303238303030383133303537313430303420202030303030393031 3630323830303038313030353731343030352020203030303035303136303238303030383042 3035373134303036202020303030353030313630323830303038304330353731343030372020 2030303030363031363032383030303831323035373134303038202020303030304130313630 3238303030383131303537313430303920202030303033353031363032383030303830433035 3731343031302020203030303435303136303238303030383131303537313430313120202030 3030353230313630323830303038304230353731343031322020203030303331303136303238 3030303830453035373134303133202020303030343830313630323830303038313330353731 3430313420202030303031453031363032383030303830453035373134303135202020303030 3343303136303238303030383130303537313430313620202030303034413031363032383030 3038313330353731343031372020203030303433303136303238303030383130303537313430 3138202020303030323730313630323830303038304630353731343031392020203030303735 3031363032383030303830453035373134303230202020303030343030313630323830303038 3043303537313430323120202030303032353031363032383030303831333035373134303232 2020203030303645303136303238303030383042303537313430323320202030303030463031 3630323830303038304130353731343032342020203030303731303136303238303030383046 301E28C0

Note: The above byte array is converted from the FFT message shown in Example 1. 


\section{Appendix B: Sample Transmission XML}

〈?xml version="1.0" encoding="UTF-8" ?>

- 〈tranBlock>

- $\langle$ tran ID="24" companyID="QGOVTEST" auxID="0580215456"〉

$-\langle T, 2.06 .0\rangle$

<eventTS>2009-08-27T20:15:27Z</eventTS>

<equipment ID="0000954197" equipType="tractor" unitAddress="0000954197" mobileType="1" / >

<position lon="-87.978055555" lat="41.7125" posTS="2009-08-27T20:15:27Z" />

〈proximity postal="60540" country="US" stateProv="IL" city="Naperville" direction="ESE" distance="9.84" placeType="CITY" />

〈proximity postal="60561" country="US" stateProv="IL" city="Darien" direction="S" distance="2.80" placeType="TOWN" />

<posType $>3<$ <posType $>$

<ignitionStatus>1</ignitionStatus>

$<$ tripStatus $>0</$ tripStatus $>$

$\langle$ tdDistance $>0.0</$ ttdistance $>$

$\langle T$ T.2.06.0 $\rangle$

$<$ trans

- $\langle$ tran ID="25" companyID="QGOVTEST" auxID="0580215456">

<eventTS>2009-08-27T20:16:56Z</eventTS>

<equipment ID="0000954197" equipType="tractor" unitAddress="0000954197" mobileType="1" / >

<position lon="-87.978055555" lat="41.7125" posTS="2009-08-27T20:15:27Z" />

<proximity postal="60540" country="US" stateProv="IL" city="Naperville" direction="ESE" distance="9.84" placeType="CIY" />

〈proximity postal="60561" country="US" stateProv="IL" city="Darien" direction="S" distance="2.80" placeType="TOWN" />

<posType $3<$ <posType $>$

<ignitionStatus $>1<$ /ignitionStatus $>$

$\langle$ tripStatus $>0<$ tripStatus $>$

$\langle\mathrm{GMH}>255</ G M H>$

<receivedTS $>2009-08-27 T 20: 17: 31 Z</$ receivedTS $>$

«msgPriority $><<$ msgPriority>

«msgBody>0082720092014365703194 0000701603C000800057031970000801603E00080005714215 0000C016037000800057142200000901603800080005703185 0000A01603D000080005714140

0000B01503800080005714210 0001601603900080005714145 0000A01603800080005703188 0000001603E000800057031910001101603C00080005714205 $0000 \mathrm{A01603700080005714225}$

00007016037000800057142550000601503 A00080005703187 $0001001603 \mathrm{~B} 0008000571420000008016037000800057031860000801603 \mathrm{E} 000800057031960000801603 \mathrm{C} 00080005714230$

0000D01603600080005714150 0000901603700080005703195 0000D01603F00080005714170 0000A01603800080005714160 00007016036000800057141650000701603700080005714155

$0000801503600080005703193000030160300008000</$ msgBody $>$

$\langle/ T .2 .01 .0\rangle$

$<$ tran>

$<$ tranBlock> 
Appendix C: Summary Recorded Data from the MiniDemo

\begin{tabular}{|c|c|c|c|c|c|c|c|c|c|c|c|}
\hline Event Time & at QC Hub & Tag ID & Tag Status & Seal & Temperature & Humidity & Shock & Battery & RSSI & Longitude & Latitude \\
\hline $8 / 28 / 0913: 40$ & $13: 44$ & 5703185 & Normal & OK & Normal $\left(24^{\circ} \mathrm{C}\right)$ & Normal $(55 \%)$ & OK & OK & 139 & -87.971667 & 41.732222 \\
\hline 8/28/09 13:45 & $13: 49$ & 5703185 & Normal & OK & Normal $\left(24^{\circ} \mathrm{C}\right)$ & Normal (55\%) & OK & OK & 139 & -87.971667 & 41.732222 \\
\hline $8 / 28 / 09$ 13:50 & 14:00 & 5703185 & Normal & OK & Normal $\left(24^{\circ} \mathrm{C}\right)$ & Normal (55\%) & OK & OK & 139 & -87.971667 & 41.732222 \\
\hline $8 / 28 / 09$ 13:55 & $14: 00$ & 5703185 & Normal & OK & Normal $\left(24^{\circ} \mathrm{C}\right)$ & Normal (55\%) & OK & $\mathrm{OK}$ & 139 & -87.971667 & 41.732222 \\
\hline $8 / 28 / 09$ 14:00 & $14: 06$ & 5703185 & Normal & OK & Normal $\left(24^{\circ} \mathrm{C}\right)$ & Normal (55\%) & OK & OK & 139 & -88.068611 & 41.684167 \\
\hline 8/28/09 14:05 & 14:11 & 5703185 & Normal & OK & Normal $\left(24^{\circ} \mathrm{C}\right)$ & Normal (55\%) & OK & OK & 139 & -88.154167 & 41.640556 \\
\hline 8/28/09 14:10 & 14:17 & 5703185 & Normal & OK & Normal $\left(24^{\circ} \mathrm{C}\right)$ & Normal (55\%) & OK & OK & 139 & -88.181111 & 41.568611 \\
\hline 8/28/09 14:15 & $14: 22$ & 5703185 & Normal & OK & Normal $\left(24^{\circ} \mathrm{C}\right)$ & Normal (55\%) & OK & OK & 139 & -88.197778 & 41.493611 \\
\hline 8/28/09 14:20 & $14: 22$ & 5703185 & Normal & OK & Normal $\left(24^{\circ} \mathrm{C}\right)$ & Normal (55\%) & OK & OK & 139 & -88.280000 & 41.462500 \\
\hline $8 / 28 / 09$ 14:25 & $14: 28$ & 5703185 & Normal & OK & Normal $\left(24^{\circ} \mathrm{C}\right)$ & Normal (55\%) & OK & $\mathrm{OK}$ & 139 & -88.371667 & 41.417500 \\
\hline 8/28/09 14:32 & $14: 34$ & 5703185 & Normal & OK & Normal $\left(24^{\circ} \mathrm{C}\right)$ & Normal (55\%) & OK & OK & 139 & -88.422778 & 41.392500 \\
\hline 8/28/09 14:35 & $14: 39$ & 5703185 & Normal & OK & Normal $\left(24^{\circ} \mathrm{C}\right)$ & Normal (55\%) & OK & OK & 139 & -88.422778 & 41.392500 \\
\hline $8 / 28 / 0914: 40$ & $14: 44$ & 5703185 & Normal & OK & Normal $\left(24^{\circ} \mathrm{C}\right)$ & Normal $(55 \%)$ & ok & OK & 139 & -88.422778 & 41.392500 \\
\hline 8/28/09 14:45 & $14: 50$ & 5703185 & Normal & OK & Normal $\left(24^{\circ} \mathrm{C}\right)$ & Normal (55\%) & OK & OK & 139 & -88.461389 & 41.378056 \\
\hline $8 / 28 / 09$ 14:50 & $14: 56$ & 5703185 & Normal & OK & Normal $\left(24^{\circ} \mathrm{C}\right)$ & Normal (55\%) & OK & OK & 139 & -88.575000 & 41.376944 \\
\hline $8 / 28 / 09$ 14:55 & 15:01 & 5703185 & Normal & OK & Normal $\left(24^{\circ} \mathrm{C}\right)$ & Normal $(54 \%)$ & OK & OK & 139 & -88.717500 & 41.375833 \\
\hline $8 / 28 / 09$ 15:00 & 15:07 & 5703185 & Normal & OK & Normal $\left(24^{\circ} \mathrm{C}\right)$ & Normal $(54 \%)$ & OK & OK & 139 & -88.792500 & 41.376944 \\
\hline $8 / 28 / 09$ 15:05 & $15: 12$ & 5703185 & Normal & OK & Normal $\left(24^{\circ} \mathrm{C}\right)$ & Normal $(54 \%)$ & ok & OK & 139 & -88.901944 & 41.368611 \\
\hline $8 / 28 / 09$ 15:10 & $15: 12$ & 5703185 & Normal & OK & Normal $\left(24^{\circ} \mathrm{C}\right)$ & Normal $(54 \%)$ & OK & OK & 139 & -89.014444 & 41.367500 \\
\hline $8 / 28 / 09$ 15:15 & $15: 18$ & 5703185 & Normal & OK & Normal $\left(24^{\circ} \mathrm{C}\right)$ & Normal $(54 \%)$ & OK & OK & 139 & -89.058333 & 41.413333 \\
\hline $8 / 28 / 0915: 20$ & $15: 23$ & 5703185 & Normal & OK & Normal $\left(24^{\circ} \mathrm{C}\right)$ & Normal $(54 \%)$ & ok & OK & 139 & -89.050833 & 41.460278 \\
\hline $8 / 28 / 0915: 25$ & $15: 29$ & 5703185 & Normal & OK & Normal $\left(24^{\circ} \mathrm{C}\right)$ & Normal (54\%) & OK & OK & 139 & -89.058333 & 41.375833 \\
\hline $8 / 28 / 09$ 15:30 & $15: 35$ & 5703185 & Normal & OK & Normal $\left(24^{\circ} \mathrm{C}\right)$ & Normal (54\%) & OK & OK & 139 & -89.125833 & 41.368611 \\
\hline $8 / 28 / 09$ 15:35 & $15: 40$ & 5703185 & Normal & OK & Normal $\left(24^{\circ} \mathrm{C}\right)$ & Normal $(54 \%)$ & OK & OK & 139 & -89.125833 & 41.368611 \\
\hline $8 / 28 / 0915: 40$ & $15: 46$ & 5703185 & Normal & OK & Normal $\left(24^{\circ} \mathrm{C}\right)$ & Normal (54\%) & OK & OK & 139 & -89.125833 & 41.368611 \\
\hline $8 / 28 / 09$ 15:45 & $15: 51$ & 5703185 & Normal & OK & Normal $\left(24^{\circ} \mathrm{C}\right)$ & Normal (54\%) & OK & OK & 139 & -89.200000 & 41.364444 \\
\hline $8 / 28 / 09$ 15:50 & $15: 57$ & 5703185 & Normal & OK & Normal $\left(24^{\circ} \mathrm{C}\right)$ & Normal $(54 \%)$ & ok & OK & 139 & -89.240556 & 41.371667 \\
\hline $8 / 28 / 09$ 16:00 & $16: 03$ & 5703185 & Normal & OK & Normal $\left(24^{\circ} \mathrm{C}\right)$ & Normal $(54 \%)$ & ok & OK & 139 & -89.458333 & 41.400833 \\
\hline 8/28/09 16:05 & $16: 13$ & 5703185 & Normal & OK & Normal $\left(24^{\circ} \mathrm{C}\right)$ & Normal (54\%) & OK & OK & 139 & -89.558333 & 41.393611 \\
\hline 8/28/09 16:11 & $16: 14$ & 5703185 & Normal & OK & Normal $\left(24^{\circ} \mathrm{C}\right)$ & Normal $(54 \%)$ & OK & OK & 139 & -89.558333 & 41.393611 \\
\hline $8 / 28 / 09$ 16:15 & 16:19 & 5703185 & Normal & OK & Normal $\left(24^{\circ} \mathrm{C}\right)$ & Normal $(54 \%)$ & OK & OK & 139 & -89.558333 & 41.393611 \\
\hline 8/28/09 16:20 & $16: 25$ & 5703185 & Normal & OK & Normal $\left(24^{\circ} \mathrm{C}\right)$ & Normal $(54 \%)$ & OK & OK & 139 & -89.558333 & 41.393611 \\
\hline $8 / 28 / 0916: 25$ & $16: 30$ & 5703185 & Normal & OK & Normal $\left(24^{\circ} \mathrm{C}\right)$ & Normal (54\%) & OK & OK & 139 & -89.623889 & 41.394722 \\
\hline $8 / 28 / 09$ 16:30 & $16: 36$ & 5703185 & Normal & OK & Normal $\left(24^{\circ} \mathrm{C}\right)$ & Normal (54\%) & OK & OK & 139 & -89.725833 & 41.387500 \\
\hline $8 / 28 / 09$ 16:35 & $16: 41$ & 5703185 & Normal & OK & Normal $\left(24^{\circ} \mathrm{C}\right)$ & Normal (54\%) & OK & OK & 139 & -89.834167 & 41.388333 \\
\hline $8 / 28 / 09$ 16:40 & $16: 47$ & 5703185 & Normal & OK & Normal $\left(24^{\circ} \mathrm{C}\right)$ & Normal $(54 \%)$ & OK & OK & 139 & -89.941667 & 41.416667 \\
\hline $8 / 28 / 09$ 16:45 & $16: 52$ & 5703185 & Normal & OK & Normal $\left(24^{\circ} \mathrm{C}\right)$ & Normal $(54 \%)$ & OK & OK & 139 & -90.050000 & 41.413333 \\
\hline 8/28/09 16:50 & $16: 52$ & 5703185 & Normal & OK & Normal $\left(24^{\circ} \mathrm{C}\right)$ & Normal (54\%) & OK & OK & 139 & -90.157222 & 41.430000 \\
\hline 8/28/09 16:55 & $16: 58$ & 5703185 & Normal & OK & Normal $\left(24^{\circ} \mathrm{C}\right)$ & Normal $(54 \%)$ & OK & OK & 139 & -90.267500 & 41.440556 \\
\hline $8 / 28 / 09$ 17:00 & 17:09 & 5703185 & Normal & OK & Normal $\left(24^{\circ} \mathrm{C}\right)$ & Normal $(54 \%)$ & OK & OK & 139 & -90.338333 & 41.476944 \\
\hline 8/28/09 17:05 & 17:09 & 5703185 & Normal & OK & Normal $\left(24^{\circ} \mathrm{C}\right)$ & Normal (54\%) & OK & OK & 139 & -90.334167 & 41.486389 \\
\hline $8 / 28 / 09$ 17:11 & $17: 15$ & 5703185 & Normal & OK & Normal $\left(24^{\circ} \mathrm{C}\right)$ & Normal $(54 \%)$ & ok & OK & 139 & -90.334167 & 41.486389 \\
\hline $8 / 28 / 09$ 17:15 & $17: 20$ & 5703185 & Normal & OK & Normal $\left(24^{\circ} \mathrm{C}\right)$ & Normal $(54 \%)$ & OK & OK & 139 & -90.334167 & 41.486389 \\
\hline $8 / 28 / 09$ 17:20 & $17: 26$ & 5703185 & Normal & OK & Normal $\left(24^{\circ} \mathrm{C}\right)$ & Normal (54\%) & OK & OK & 139 & -90.334167 & 41.486389 \\
\hline $8 / 28 / 09$ 17:25 & $17: 31$ & 5703185 & Normal & OK & Normal $\left(24^{\circ} \mathrm{C}\right)$ & Normal $(54 \%)$ & OK & OK & 139 & -90.340556 & 41.511389 \\
\hline $8 / 28 / 09$ 17:30 & $17: 37$ & 5703185 & Normal & OK & Normal $\left(24^{\circ} \mathrm{C}\right)$ & Normal $(54 \%)$ & OK & OK & 139 & -90.234167 & 41.562500 \\
\hline $8 / 28 / 09$ 17:35 & $17: 42$ & 5703185 & Normal & OK & Normal $\left(24^{\circ} \mathrm{C}\right)$ & Normal $(54 \%)$ & OK & OK & 139 & -90.209167 & 41.581111 \\
\hline $8 / 28 / 0917: 40$ & $17: 42$ & 5703185 & Normal & OK & Normal $\left(24^{\circ} \mathrm{C}\right)$ & Normal $(54 \%)$ & OK & OK & 139 & -90.163333 & 41.642500 \\
\hline $8 / 28 / 09$ 17:45 & $17: 48$ & 5703185 & Normal & OK & Normal $\left(24^{\circ} \mathrm{C}\right)$ & Normal (54\%) & OK & OK & 139 & -90.080000 & 41.690556 \\
\hline $8 / 28 / 09$ 17:50 & $17: 53$ & 5703185 & Normal & OK & Normal $\left(24^{\circ} \mathrm{C}\right)$ & Normal $(54 \%)$ & OK & OK & 139 & -89.983333 & 41.718611 \\
\hline $8 / 28 / 09$ 17:55 & $17: 59$ & 5703185 & Normal & OK & Normal $\left(26^{\circ} \mathrm{C}\right)$ & Normal $(54 \%)$ & OK & OK & 139 & -89.876944 & 41.744722 \\
\hline $8 / 28 / 09$ 18:00 & 18:04 & 5703185 & Normal & OK & Normal $\left(26^{\circ} \mathrm{C}\right)$ & Normal $(54 \%)$ & OK & OK & 139 & -89.775000 & 41.757222 \\
\hline 8/28/09 18:05 & $18: 10$ & 5703185 & Normal & OK & Normal $\left(26^{\circ} \mathrm{C}\right)$ & Normal $(54 \%)$ & OK & OK & 139 & -89.689444 & 41.750833 \\
\hline $8 / 28 / 09$ 18:10 & $18: 15$ & 5703185 & Normal & OK & Normal $\left(26^{\circ} \mathrm{C}\right)$ & Normal $(54 \%)$ & OK & OK & 139 & -89.689444 & 41.762500 \\
\hline 8/28/09 18:15 & $18: 21$ & 5703185 & Normal & OK & Normal $\left(26^{\circ} \mathrm{C}\right)$ & Normal (54\%) & OK & OK & 139 & -89.678056 & 41.750833 \\
\hline $8 / 28 / 09$ 18:20 & $18: 26$ & 5703185 & Normal & OK & Normal $\left(26^{\circ} \mathrm{C}\right)$ & Normal $(54 \%)$ & OK & OK & 139 & -89.635278 & 41.759167 \\
\hline $8 / 28 / 09$ 18:25 & $18: 32$ & 5703185 & Normal & OK & Normal $\left(26^{\circ} \mathrm{C}\right)$ & Normal $(54 \%)$ & OK & OK & 139 & -89.532222 & 41.789444 \\
\hline $8 / 28 / 09$ 18:30 & $18: 38$ & 5703185 & Normal & OK & Normal $\left(26^{\circ} \mathrm{C}\right)$ & Normal (54\%) & OK & OK & 139 & -89.432222 & 41.822778 \\
\hline $8 / 28 / 09$ 18:35 & $18: 38$ & 5703185 & Normal & OK & Normal $\left(26^{\circ} \mathrm{C}\right)$ & Normal $(54 \%)$ & OK & OK & 139 & -89.336389 & 41.822778 \\
\hline $8 / 28 / 09$ 18:40 & $18: 43$ & 5703185 & Normal & OK & Normal $\left(26^{\circ} \mathrm{C}\right)$ & Normal $(54 \%)$ & OK & OK & 139 & -89.231111 & 41.850833 \\
\hline 8/28/09 18:45 & $18: 48$ & 5703185 & Normal & OK & Normal $\left(26^{\circ} \mathrm{C}\right)$ & Normal (54\%) & OK & OK & 139 & -89.125000 & 41.883333 \\
\hline $8 / 28 / 09$ 18:50 & $18: 54$ & 5703185 & Normal & OK & Normal $\left(26^{\circ} \mathrm{C}\right)$ & Normal (54\%) & OK & OK & 139 & -89.025000 & 41.904167 \\
\hline $8 / 28 / 09$ 18:55 & 19:00 & 5703185 & Normal & OK & Normal $\left(26^{\circ} \mathrm{C}\right)$ & Normal (54\%) & OK & OK & 139 & -88.913333 & 41.900000 \\
\hline 8/28/09 19:00 & 19:05 & 5703185 & Normal & OK & Normal $\left(26^{\circ} \mathrm{C}\right)$ & Normal $(54 \%)$ & OK & OK & 139 & -88.814444 & 41.900000 \\
\hline 8/28/09 19:05 & 19:11 & 5703185 & Normal & OK & Normal $\left(26^{\circ} \mathrm{C}\right)$ & Normal $(54 \%)$ & OK & OK & 139 & -88.739444 & 41.900000 \\
\hline 8/28/09 19:10 & 19:16 & 5703185 & Normal & OK & Normal $\left(26^{\circ} \mathrm{C}\right)$ & Normal (52\%) & OK & OK & 139 & -88.739444 & 41.900000 \\
\hline $8 / 28 / 09$ 19:15 & 19:22 & 5703185 & Normal & OK & Normal $\left(26^{\circ} \mathrm{C}\right)$ & Normal $(52 \%)$ & ok & OK & 139 & -88.737500 & 41.900000 \\
\hline $8 / 28 / 09$ 19:20 & $19: 27$ & 5703185 & Normal & OK & Normal $\left(26^{\circ} \mathrm{C}\right)$ & Normal (52\%) & OK & OK & 139 & -88.737500 & 41.900000 \\
\hline $8 / 28 / 09$ 19:25 & 19:33 & 5703185 & Normal & OK & Normal $\left(26^{\circ} \mathrm{C}\right)$ & Normal $(52 \%)$ & OK & OK & 139 & -88.639444 & 41.894722 \\
\hline $8 / 28 / 09$ 19:30 & 19:38 & 5703185 & Normal & OK & Normal $\left(26^{\circ} \mathrm{C}\right)$ & Normal $(52 \%)$ & OK & OK & 139 & -88.538333 & 41.859167 \\
\hline 8/28/09 19:35 & 19:38 & 5703185 & Normal & OK & Normal $\left(26^{\circ} \mathrm{C}\right)$ & Normal $(52 \%)$ & OK & OK & 139 & -88.448889 & 41.812500 \\
\hline $8 / 28 / 09$ 19:40 & $19: 44$ & 5703185 & Normal & OK & Normal $\left(26^{\circ} \mathrm{C}\right)$ & Normal $(52 \%)$ & OK & OK & 139 & -88.347778 & 41.794722 \\
\hline 8/28/09 19:45 & 19:49 & 5703185 & Normal & OK & Normal $\left(26^{\circ} \mathrm{C}\right)$ & Normal $(52 \%)$ & OK & OK & 139 & -88.261389 & 41.800000 \\
\hline $8 / 28 / 09$ 19:50 & 19:55 & 5703185 & Normal & OK & Normal $\left(26^{\circ} \mathrm{C}\right)$ & Normal $(52 \%)$ & OK & OK & 139 & -88.156111 & 41.806111 \\
\hline 8/28/09 19:55 & 20:00 & 5703185 & Normal & OK & Normal $\left(26^{\circ} \mathrm{C}\right)$ & Normal $(52 \%)$ & OK & OK & 139 & -88.062500 & 41.810278 \\
\hline 8/28/09 20:00 & 20:06 & 5703185 & Normal & OK & Normal $\left(26^{\circ} \mathrm{C}\right)$ & Normal $(52 \%)$ & OK & OK & 139 & -88.054167 & 41.807222 \\
\hline 8/28/09 20:05 & 20:11 & 5703185 & Normal & OK & Normal $\left(26^{\circ} \mathrm{C}\right)$ & Normal $(53 \%)$ & OK & OK & 139 & -88.054167 & 41.781111 \\
\hline
\end{tabular}




\begin{tabular}{|c|c|c|c|c|c|c|c|c|c|c|c|}
\hline Event Time & at QC Hub & Tag ID & Tag Status & Seal & Temperature & Humidity & Shock & Battery & RSSI & Longitude & Latitude \\
\hline $8 / 28 / 0920: 10$ & $20: 22$ & 5703185 & Normal & OK & Normal $\left(26^{\circ} \mathrm{C}\right)$ & Normal (53\%) & OK & OK & 139 & -88.035278 & 41.732222 \\
\hline $8 / 28 / 0913: 40$ & $13: 44$ & 5703186 & Normal & OK & Normal $\left(23^{\circ} \mathrm{C}\right)$ & Normal (54\%) & OK & $\mathrm{OK}$ & 140 & -87.971667 & 41.732222 \\
\hline 8/28/09 13:45 & $13: 49$ & 5703186 & Normal & OK & Normal $\left(23^{\circ} \mathrm{C}\right)$ & Normal (54\%) & $\mathrm{OK}$ & OK & 140 & -87.971667 & 41.732222 \\
\hline 8/28/09 13:50 & $14: 00$ & 5703186 & Normal & OK & Normal $\left(23^{\circ} \mathrm{C}\right)$ & Normal (54\%) & $\mathrm{OK}$ & OK & 140 & -87.971667 & 41.732222 \\
\hline 8/28/09 13:55 & $14: 00$ & 5703186 & Normal & $\mathrm{OK}$ & Normal $\left(23^{\circ} \mathrm{C}\right)$ & Normal (54\%) & OK & OK & 140 & -87.971667 & 41.732222 \\
\hline 8/28/09 14:00 & $14: 06$ & 5703186 & Normal & OK & Normal $\left(23^{\circ} \mathrm{C}\right)$ & Normal (54\%) & OK & OK & 140 & -88.068611 & 41.684167 \\
\hline 8/28/09 14:05 & 14:11 & 5703186 & Normal & $\mathrm{OK}$ & Normal $\left(23^{\circ} \mathrm{C}\right)$ & Normal (54\%) & OK & $\mathrm{OK}$ & 140 & -88.154167 & 41.640556 \\
\hline 8/28/09 14:10 & $14: 17$ & 5703186 & Normal & $\mathrm{OK}$ & Normal $\left(23^{\circ} \mathrm{C}\right)$ & Normal (54\%) & OK & OK & 140 & -88.181111 & 41.568611 \\
\hline 8/28/09 14:15 & $14: 22$ & 5703186 & Normal & OK & Normal $\left(23^{\circ} \mathrm{C}\right)$ & Normal (54\%) & OK & OK & 140 & -88.197778 & 41.493611 \\
\hline 8/28/09 14:20 & $14: 22$ & 5703186 & Normal & $\mathrm{OK}$ & Normal $\left(23^{\circ} \mathrm{C}\right)$ & Normal (54\%) & OK & OK & 140 & -88.280000 & 41.462500 \\
\hline $8 / 28 / 0914: 25$ & $14: 28$ & 5703186 & Normal & $\mathrm{OK}$ & Normal $\left(23^{\circ} \mathrm{C}\right)$ & Normal (54\%) & OK & OK & 140 & -88.371667 & 41.417500 \\
\hline $8 / 28 / 0914: 32$ & $14: 34$ & 5703186 & Normal & OK & Normal $\left(23^{\circ} \mathrm{C}\right)$ & Normal (54\%) & OK & OK & 140 & -88.422778 & 41.392500 \\
\hline 8/28/09 14:35 & $14: 39$ & 5703186 & Normal & $\mathrm{OK}$ & Normal $\left(23^{\circ} \mathrm{C}\right)$ & Normal (54\%) & OK & OK & 140 & -88.422778 & 41.392500 \\
\hline 8/28/09 14:40 & $14: 44$ & 5703186 & Normal & OK & Normal $\left(23^{\circ} \mathrm{C}\right)$ & Normal (54\%) & OK & OK & 140 & -88.422778 & 41.392500 \\
\hline 8/28/09 14:45 & $14: 50$ & 5703186 & Normal & OK & Normal $\left(23^{\circ} \mathrm{C}\right)$ & Normal (54\%) & OK & OK & 140 & -88.461389 & 41.378056 \\
\hline 8/28/09 14:50 & $14: 56$ & 5703186 & Normal & OK & Normal $\left(23^{\circ} \mathrm{C}\right)$ & Normal (54\%) & OK & OK & 140 & -88.575000 & 41.376944 \\
\hline 8/28/09 14:55 & 15:01 & 5703186 & Normal & OK & Normal $\left(24^{\circ} \mathrm{C}\right)$ & Normal (54\%) & $\mathrm{OK}$ & OK & 140 & -88.717500 & 41.375833 \\
\hline 8/28/09 15:00 & $15: 07$ & 5703186 & Normal & OK & Normal $\left(24^{\circ} \mathrm{C}\right)$ & Normal (54\%) & OK & OK & 140 & -88.792500 & 41.376944 \\
\hline 8/28/09 15:05 & $15: 12$ & 5703186 & Normal & OK & Normal $\left(24^{\circ} \mathrm{C}\right)$ & Normal (54\%) & OK & OK & 140 & -88.901944 & 41.368611 \\
\hline 8/28/09 15:10 & $15: 12$ & 5703186 & Normal & $\mathrm{OK}$ & Normal $\left(24^{\circ} \mathrm{C}\right)$ & Normal (54\%) & OK & OK & 140 & -89.014444 & 41.367500 \\
\hline 8/28/09 15:15 & $15: 18$ & 5703186 & Normal & $\mathrm{OK}$ & Normal $\left(24^{\circ} \mathrm{C}\right)$ & Normal (54\%) & OK & OK & 140 & -89.058333 & 41.413333 \\
\hline $8 / 28 / 09$ 15:20 & $15: 23$ & 5703186 & Normal & OK & Normal $\left(24^{\circ} \mathrm{C}\right)$ & Normal (54\%) & OK & OK & 140 & -89.050833 & 41.460278 \\
\hline $8 / 28 / 09$ 15:25 & $15: 29$ & 5703186 & Normal & OK & Normal $\left(24^{\circ} \mathrm{C}\right)$ & Normal (54\%) & OK & OK & 140 & -89.058333 & 41.375833 \\
\hline 8/28/09 15:30 & $15: 35$ & 5703186 & Normal & OK & Normal $\left(24^{\circ} \mathrm{C}\right)$ & Normal (54\%) & OK & OK & 140 & -89.125833 & 41.368611 \\
\hline 8/28/09 15:35 & $15: 40$ & 5703186 & Normal & OK & Normal $\left(24^{\circ} \mathrm{C}\right)$ & Normal $(54 \%)$ & OK & OK & 140 & -89.125833 & 41.368611 \\
\hline 8/28/09 15:40 & $15: 46$ & 5703186 & Normal & OK & Normal $\left(24^{\circ} \mathrm{C}\right)$ & Normal (54\%) & OK & OK & 140 & -89.125833 & 41.368611 \\
\hline 8/28/09 15:45 & $15: 51$ & 5703186 & Normal & OK & Normal $\left(24^{\circ} \mathrm{C}\right)$ & Normal (54\%) & OK & OK & 140 & -89.200000 & 41.364444 \\
\hline 8/28/09 15:50 & $15: 57$ & 5703186 & Normal & OK & Normal $\left(24^{\circ} \mathrm{C}\right)$ & Normal (54\%) & OK & OK & 140 & -89.240556 & 41.371667 \\
\hline 8/28/09 16:00 & $16: 03$ & 5703186 & Normal & OK & Normal $\left(24^{\circ} \mathrm{C}\right)$ & Normal (54\%) & OK & OK & 140 & -89.458333 & 41.400833 \\
\hline 8/28/09 16:05 & $16: 13$ & 5703186 & Normal & OK & Normal $\left(24^{\circ} \mathrm{C}\right)$ & Normal (54\%) & OK & OK & 140 & -89.558333 & 41.393611 \\
\hline 8/28/09 16:11 & $16: 14$ & 5703186 & Normal & OK & Normal $\left(24^{\circ} \mathrm{C}\right)$ & Normal $(54 \%)$ & OK & OK & 140 & -89.558333 & 41.393611 \\
\hline 8/28/09 16:15 & $16: 19$ & 5703186 & Normal & OK & Normal $\left(24^{\circ} \mathrm{C}\right)$ & Normal (54\%) & OK & OK & 140 & -89.558333 & 41.393611 \\
\hline 8/28/09 16:20 & $16: 25$ & 5703186 & Normal & OK & Normal $\left(24^{\circ} \mathrm{C}\right)$ & Normal (54\%) & OK & OK & 140 & -89.558333 & 41.393611 \\
\hline $8 / 28 / 0916: 25$ & $16: 30$ & 5703186 & Normal & OK & Normal $\left(24^{\circ} \mathrm{C}\right)$ & Normal (54\%) & OK & OK & 140 & -89.623889 & 41.394722 \\
\hline 8/28/09 16:30 & $16: 36$ & 5703186 & Normal & OK & Normal $\left(24^{\circ} \mathrm{C}\right)$ & Normal $(54 \%)$ & OK & OK & 140 & -89.725833 & 41.387500 \\
\hline 8/28/09 16:35 & $16: 41$ & 5703186 & Normal & OK & Normal $\left(24^{\circ} \mathrm{C}\right)$ & Normal (54\%) & OK & OK & 140 & -89.834167 & 41.388333 \\
\hline 8/28/09 16:40 & $16: 47$ & 5703186 & Normal & OK & Normal $\left(24^{\circ} \mathrm{C}\right)$ & Normal (54\%) & OK & OK & 140 & -89.941667 & 41.416667 \\
\hline 8/28/09 16:45 & $16: 52$ & 5703186 & Normal & OK & Normal $\left(24^{\circ} \mathrm{C}\right)$ & Normal $(54 \%)$ & OK & OK & 140 & -90.050000 & 41.413333 \\
\hline 8/28/09 16:50 & $16: 52$ & 5703186 & Normal & OK & Normal $\left(24^{\circ} \mathrm{C}\right)$ & Normal $(54 \%)$ & OK & OK & 140 & -90.157222 & 41.430000 \\
\hline 8/28/09 16:55 & $16: 58$ & 5703186 & Normal & OK & Normal $\left(24^{\circ} \mathrm{C}\right)$ & Normal (54\%) & OK & OK & 140 & -90.267500 & 41.440556 \\
\hline 8/28/09 17:00 & 17:09 & 5703186 & Normal & OK & Normal $\left(24^{\circ} \mathrm{C}\right)$ & Normal (54\%) & OK & OK & 140 & -90.338333 & 41.476944 \\
\hline 8/28/09 17:05 & 17:09 & 5703186 & Normal & OK & Normal $\left(24^{\circ} \mathrm{C}\right)$ & Normal (54\%) & OK & OK & 140 & -90.334167 & 41.486389 \\
\hline 8/28/09 17:11 & $17: 15$ & 5703186 & Normal & OK & Normal $\left(24^{\circ} \mathrm{C}\right)$ & Normal (54\%) & OK & OK & 140 & -90.334167 & 41.486389 \\
\hline 8/28/09 17:15 & $17: 20$ & 5703186 & Normal & OK & Normal $\left(24^{\circ} \mathrm{C}\right)$ & Normal $(54 \%)$ & OK & OK & 140 & -90.334167 & 41.486389 \\
\hline 8/28/09 17:20 & $17: 26$ & 5703186 & Normal & OK & Normal $\left(24^{\circ} \mathrm{C}\right)$ & Normal (54\%) & OK & OK & 140 & -90.334167 & 41.486389 \\
\hline 8/28/09 17:25 & $17: 31$ & 5703186 & Normal & OK & Normal $\left(24^{\circ} \mathrm{C}\right)$ & Normal $(54 \%)$ & OK & OK & 140 & -90.340556 & 41.511389 \\
\hline 8/28/09 17:30 & $17: 37$ & 5703186 & Normal & OK & Normal $\left(24^{\circ} \mathrm{C}\right)$ & Normal $(54 \%)$ & OK & OK & 140 & -90.234167 & 41.562500 \\
\hline 8/28/09 17:35 & $17: 42$ & 5703186 & Normal & OK & Normal $\left(24^{\circ} \mathrm{C}\right)$ & Normal $(54 \%)$ & OK & OK & 140 & -90.209167 & 41.581111 \\
\hline 8/28/09 17:40 & $17: 42$ & 5703186 & Normal & OK & Normal $\left(24^{\circ} \mathrm{C}\right)$ & Normal (54\%) & OK & OK & 140 & -90.163333 & 41.642500 \\
\hline 8/28/09 17:45 & $17: 48$ & 5703186 & Normal & OK & Normal $\left(24^{\circ} \mathrm{C}\right)$ & Normal $(54 \%)$ & OK & OK & 140 & -90.080000 & 41.690556 \\
\hline 8/28/09 17:50 & $17: 53$ & 5703186 & Normal & OK & Normal $\left(24^{\circ} \mathrm{C}\right)$ & Normal (54\%) & OK & OK & 140 & -89.983333 & 41.718611 \\
\hline 8/28/09 17:55 & $17: 59$ & 5703186 & Normal & OK & Normal $\left(26^{\circ} \mathrm{C}\right)$ & Normal $(52 \%)$ & OK & OK & 140 & -89.876944 & 41.744722 \\
\hline 8/28/09 18:00 & $18: 04$ & 5703186 & Normal & OK & Normal $\left(26^{\circ} \mathrm{C}\right)$ & Normal $(52 \%)$ & OK & OK & 140 & -89.775000 & 41.757222 \\
\hline 8/28/09 18:05 & 18:10 & 5703186 & Normal & OK & Normal $\left(26^{\circ} \mathrm{C}\right)$ & Normal $(52 \%)$ & OK & OK & 140 & -89.689444 & 41.750833 \\
\hline 8/28/09 18:10 & $18: 15$ & 5703186 & Normal & OK & Normal $\left(26^{\circ} \mathrm{C}\right)$ & Normal $(52 \%)$ & OK & OK & 140 & -89.689444 & 41.762500 \\
\hline 8/28/09 18:15 & $18: 21$ & 5703186 & Normal & OK & Normal $\left(26^{\circ} \mathrm{C}\right)$ & Normal $(52 \%)$ & OK & OK & 140 & -89.678056 & 41.750833 \\
\hline 8/28/09 18:20 & $18: 26$ & 5703186 & Normal & OK & Normal $\left(26^{\circ} \mathrm{C}\right)$ & Normal $(52 \%)$ & OK & OK & 140 & -89.635278 & 41.759167 \\
\hline 8/28/09 18:25 & $18: 32$ & 5703186 & Normal & OK & Normal $\left(26^{\circ} \mathrm{C}\right)$ & Normal $(52 \%)$ & OK & OK & 140 & -89.532222 & 41.789444 \\
\hline 8/28/09 18:30 & $18: 38$ & 5703186 & Normal & OK & Normal $\left(26^{\circ} \mathrm{C}\right)$ & Normal $(52 \%)$ & OK & OK & 140 & -89.432222 & 41.822778 \\
\hline 8/28/09 18:35 & $18: 38$ & 5703186 & Normal & OK & Normal $\left(26^{\circ} \mathrm{C}\right)$ & Normal $(52 \%)$ & OK & OK & 140 & -89.336389 & 41.822778 \\
\hline 8/28/09 18:40 & $18: 43$ & 5703186 & Normal & OK & Normal $\left(26^{\circ} \mathrm{C}\right)$ & Normal (52\%) & OK & OK & 140 & -89.231111 & 41.850833 \\
\hline 8/28/09 18:45 & $18: 48$ & 5703186 & Normal & OK & Normal $\left(27^{\circ} \mathrm{C}\right)$ & Normal $(50 \%)$ & OK & OK & 140 & -89.125000 & 41.883333 \\
\hline 8/28/09 18:50 & $18: 54$ & 5703186 & Normal & OK & Normal $\left(27^{\circ} \mathrm{C}\right)$ & Normal $(50 \%)$ & OK & OK & 140 & -89.025000 & 41.904167 \\
\hline 8/28/09 18:55 & 19:00 & 5703186 & Normal & OK & Normal $\left(27^{\circ} \mathrm{C}\right)$ & Normal $(50 \%)$ & OK & OK & 140 & -88.913333 & 41.900000 \\
\hline 8/28/09 19:00 & 19:05 & 5703186 & Normal & OK & Normal $\left(27^{\circ} \mathrm{C}\right)$ & Normal $(50 \%)$ & OK & OK & 140 & -88.814444 & 41.900000 \\
\hline 8/28/09 19:05 & 19:11 & 5703186 & Normal & OK & Normal $\left(27^{\circ} \mathrm{C}\right)$ & Normal $(50 \%)$ & OK & OK & 140 & -88.739444 & 41.900000 \\
\hline 8/28/09 19:10 & 19:16 & 5703186 & Normal & OK & Normal $\left(27^{\circ} \mathrm{C}\right)$ & Normal $(50 \%)$ & OK & OK & 140 & -88.739444 & 41.900000 \\
\hline 8/28/09 19:15 & 19:22 & 5703186 & Normal & OK & Normal $\left(27^{\circ} \mathrm{C}\right)$ & Normal $(49 \%)$ & OK & OK & 140 & -88.737500 & 41.900000 \\
\hline $8 / 28 / 09$ 19:20 & 19:27 & 5703186 & Normal & OK & Normal $\left(27^{\circ} \mathrm{C}\right)$ & Normal (49\%) & OK & OK & 140 & -88.737500 & 41.900000 \\
\hline $8 / 28 / 09$ 19:25 & 19:33 & 5703186 & Normal & OK & Normal $\left(27^{\circ} \mathrm{C}\right)$ & Normal (49\%) & OK & OK & 140 & -88.639444 & 41.894722 \\
\hline 8/28/09 19:30 & 19:38 & 5703186 & Normal & OK & Normal $\left(27^{\circ} \mathrm{C}\right)$ & Normal (49\%) & OK & OK & 140 & -88.538333 & 41.859167 \\
\hline 8/28/09 19:35 & 19:38 & 5703186 & Normal & OK & Normal $\left(27^{\circ} \mathrm{C}\right)$ & Normal (49\%) & OK & OK & 140 & -88.448889 & 41.812500 \\
\hline 8/28/09 19:40 & $19: 44$ & 5703186 & Normal & OK & Normal $\left(27^{\circ} \mathrm{C}\right)$ & Normal (49\%) & OK & OK & 140 & -88.347778 & 41.794722 \\
\hline 8/28/09 19:45 & $19: 49$ & 5703186 & Normal & OK & Normal $\left(27^{\circ} \mathrm{C}\right)$ & Normal (49\%) & OK & OK & 140 & -88.261389 & 41.800000 \\
\hline 8/28/09 19:50 & 19:55 & 5703186 & Normal & OK & Normal $\left(27^{\circ} \mathrm{C}\right)$ & Normal (49\%) & OK & OK & 140 & -88.156111 & 41.806111 \\
\hline 8/28/09 19:55 & $20: 00$ & 5703186 & Normal & OK & Normal $\left(27^{\circ} \mathrm{C}\right)$ & Normal (49\%) & OK & OK & 140 & -88.062500 & 41.810278 \\
\hline 8/28/09 20:00 & 20:06 & 5703186 & Normal & OK & Normal $\left(27^{\circ} \mathrm{C}\right)$ & Normal (49\%) & OK & OK & 140 & -88.054167 & 41.807222 \\
\hline 8/28/09 20:05 & $20: 11$ & 5703186 & Normal & OK & Normal $\left(27^{\circ} \mathrm{C}\right)$ & Normal $(50 \%)$ & OK & OK & 140 & -88.054167 & 41.781111 \\
\hline 8/28/09 20:10 & $20: 22$ & 5703186 & Normal & $\mathrm{OK}$ & Normal $\left(27^{\circ} \mathrm{C}\right)$ & Normal $(50 \%)$ & $\mathrm{OK}$ & OK & 140 & -88.035278 & 41.732222 \\
\hline 8/28/09 13:40 & $13: 44$ & 5703187 & Normal & OK & Normal $\left(23^{\circ} \mathrm{C}\right)$ & Normal $(55 \%)$ & OK & $\mathrm{OK}$ & 140 & -87.971667 & 41.732222 \\
\hline 8/28/09 13:45 & $13: 49$ & 5703187 & Normal & OK & Normal $\left(23^{\circ} \mathrm{C}\right)$ & Normal (55\%) & OK & OK & 140 & -87.971667 & 41.732222 \\
\hline
\end{tabular}




\begin{tabular}{|c|c|c|c|c|c|c|c|c|c|c|c|}
\hline Event Time & at QC Hub & Tag ID & Tag Status & Seal & Temperature & Humidity & Shock & Battery & RSSI & Longitude & Latitude \\
\hline $8 / 28 / 0913: 50$ & $14: 00$ & 5703187 & Normal & $\mathrm{OK}$ & Normal $\left(23^{\circ} \mathrm{C}\right)$ & Normal $(55 \%)$ & OK & OK & 140 & -87.971667 & 41.732222 \\
\hline 8/28/09 13:55 & $14: 00$ & 5703187 & Normal & OK & Normal $\left(23^{\circ} \mathrm{C}\right)$ & Normal (55\%) & $\mathrm{OK}$ & OK & 140 & -87.971667 & 41.732222 \\
\hline 8/28/09 14:00 & $14: 06$ & 5703187 & Normal & OK & Normal $\left(23^{\circ} \mathrm{C}\right)$ & Normal (55\%) & OK & OK & 140 & -88.068611 & 41.684167 \\
\hline 8/28/09 14:05 & $14: 11$ & 5703187 & Normal & OK & Normal $\left(23^{\circ} \mathrm{C}\right)$ & Normal (55\%) & $\mathrm{OK}$ & OK & 140 & -88.154167 & 41.640556 \\
\hline 8/28/09 14:10 & $14: 17$ & 5703187 & Normal & OK & Normal $\left(23^{\circ} \mathrm{C}\right)$ & Normal (55\%) & $\mathrm{OK}$ & OK & 140 & -88.181111 & 41.568611 \\
\hline 8/28/09 14:15 & $14: 22$ & 5703187 & Normal & $\mathrm{OK}$ & Normal $\left(23^{\circ} \mathrm{C}\right)$ & Normal (55\%) & OK & OK & 140 & -88.197778 & 41.493611 \\
\hline $8 / 28 / 0914: 20$ & $14: 22$ & 5703187 & Normal & $\mathrm{OK}$ & Normal $\left(23^{\circ} \mathrm{C}\right)$ & Normal (55\%) & $\mathrm{OK}$ & OK & 140 & -88.280000 & 41.462500 \\
\hline 8/28/09 14:25 & $14: 28$ & 5703187 & Normal & OK & Normal $\left(23^{\circ} \mathrm{C}\right)$ & Normal (55\%) & OK & OK & 140 & -88.371667 & 41.417500 \\
\hline $8 / 28 / 09$ 14:32 & $14: 34$ & 5703187 & Normal & $\mathrm{OK}$ & Normal $\left(23^{\circ} \mathrm{C}\right)$ & Normal (55\%) & OK & OK & 140 & -88.422778 & 41.392500 \\
\hline $8 / 28 / 09$ 14:35 & $14: 39$ & 5703187 & Normal & OK & Normal $\left(23^{\circ} \mathrm{C}\right)$ & Normal (55\%) & OK & OK & 140 & -88.422778 & 41.392500 \\
\hline $8 / 28 / 0914: 40$ & $14: 44$ & 5703187 & Normal & OK & Normal $\left(23^{\circ} \mathrm{C}\right)$ & Normal (55\%) & OK & OK & 140 & -88.422778 & 41.392500 \\
\hline $8 / 28 / 09$ 14:45 & $14: 50$ & 5703187 & Normal & OK & Normal $\left(23^{\circ} \mathrm{C}\right)$ & Normal (55\%) & OK & OK & 140 & -88.461389 & 41.37805 \\
\hline $8 / 28 / 09$ 14:50 & $14: 56$ & 5703187 & Normal & OK & Normal $\left(23^{\circ} \mathrm{C}\right)$ & Normal (55\%) & OK & OK & 140 & -88.575000 & 41.37694 \\
\hline 8/28/09 14:55 & $15: 01$ & 5703187 & Normal & OK & Normal $\left(24^{\circ} \mathrm{C}\right)$ & Normal (54\%) & $\mathrm{OK}$ & OK & 140 & -88.717500 & 41.375833 \\
\hline 8/28/09 15:00 & $15: 07$ & 5703187 & Normal & OK & Normal $\left(24^{\circ} \mathrm{C}\right)$ & Normal (54\%) & OK & OK & 140 & -88.792500 & 41.37694 \\
\hline 8/28/09 15:05 & $15: 12$ & 5703187 & Normal & OK & Normal $\left(24^{\circ} \mathrm{C}\right)$ & Normal (54\%) & OK & OK & 140 & -88.901944 & 41.368611 \\
\hline 8/28/09 15:10 & $15: 12$ & 5703187 & Normal & $\mathrm{OK}$ & Normal $\left(24^{\circ} \mathrm{C}\right)$ & Normal (54\%) & $\mathrm{OK}$ & OK & 140 & -89.014444 & 41.367500 \\
\hline 8/28/09 15:15 & $15: 18$ & 5703187 & Normal & OK & Normal $\left(24^{\circ} \mathrm{C}\right)$ & Normal (54\%) & $\mathrm{OK}$ & OK & 140 & -89.058333 & 41.413333 \\
\hline $8 / 28 / 09$ 15:20 & $15: 23$ & 5703187 & Normal & OK & Normal $\left(24^{\circ} \mathrm{C}\right)$ & Normal (54\%) & OK & OK & 140 & -89.050833 & 41.460278 \\
\hline 8/28/09 15:25 & $15: 29$ & 5703187 & Normal & OK & Normal $\left(24^{\circ} \mathrm{C}\right)$ & Normal (54\%) & OK & OK & 140 & -89.058333 & 41.375833 \\
\hline 8/28/09 15:30 & $15: 35$ & 5703187 & Normal & OK & Normal $\left(24^{\circ} \mathrm{C}\right)$ & Normal (54\%) & $\mathrm{OK}$ & OK & 140 & -89.125833 & 41.368611 \\
\hline 8/28/09 15:35 & $15: 40$ & 5703187 & Normal & OK & Normal $\left(24^{\circ} \mathrm{C}\right)$ & Normal (54\%) & $\mathrm{OK}$ & OK & 140 & -89.125833 & 41.368611 \\
\hline $8 / 28 / 09$ 15:40 & $15: 46$ & 5703187 & Normal & OK & Normal $\left(24^{\circ} \mathrm{C}\right)$ & Normal (54\%) & OK & OK & 140 & -89.125833 & 41.368611 \\
\hline 8/28/09 15:45 & $15: 51$ & 5703187 & Normal & OK & Normal $\left(24^{\circ} \mathrm{C}\right)$ & Normal (54\%) & OK & OK & 140 & -89.200000 & 41.364444 \\
\hline $8 / 28 / 09$ 15:50 & $15: 57$ & 5703187 & Normal & OK & Normal $\left(24^{\circ} \mathrm{C}\right)$ & Normal (54\%) & OK & OK & 140 & -89.240556 & 41.371667 \\
\hline $8 / 28 / 09$ 16:00 & $16: 03$ & 5703187 & Normal & OK & Normal $\left(24^{\circ} \mathrm{C}\right)$ & Normal (54\%) & OK & OK & 140 & -89.458333 & 41.400833 \\
\hline 8/28/09 16:05 & $16: 13$ & 5703187 & Normal & OK & Normal $\left(24^{\circ} \mathrm{C}\right)$ & Normal (54\%) & OK & OK & 140 & -89.558333 & 41.393611 \\
\hline 8/28/09 16:11 & $16: 14$ & 5703187 & Normal & OK & Normal $\left(24^{\circ} \mathrm{C}\right)$ & Normal (54\%) & OK & OK & 140 & -89.558333 & 41.393611 \\
\hline 8/28/09 16:15 & $16: 19$ & 5703187 & Normal & OK & Normal $\left(24^{\circ} \mathrm{C}\right)$ & Normal (54\%) & OK & OK & 140 & -89.558333 & 41.393611 \\
\hline $8 / 28 / 09$ 16:20 & $16: 25$ & 5703187 & Normal & OK & Normal $\left(24^{\circ} \mathrm{C}\right)$ & Normal (54\%) & OK & OK & 140 & -89.558333 & 41.393611 \\
\hline $8 / 28 / 09$ 16:25 & $16: 30$ & 5703187 & Normal & OK & Normal $\left(24^{\circ} \mathrm{C}\right)$ & Normal (54\%) & OK & OK & 140 & -89.623889 & 41.394722 \\
\hline $8 / 28 / 09$ 16:30 & $16: 36$ & 5703187 & Normal & OK & Normal $\left(24^{\circ} \mathrm{C}\right)$ & Normal (54\%) & OK & OK & 140 & -89.725833 & 41.387500 \\
\hline $8 / 28 / 09$ 16:35 & $16: 41$ & 5703187 & Normal & OK & Normal $\left(24^{\circ} \mathrm{C}\right)$ & Normal $(54 \%)$ & OK & OK & 140 & -89.834167 & 41.388333 \\
\hline $8 / 28 / 0916: 40$ & $16: 47$ & 5703187 & Normal & OK & Normal $\left(24^{\circ} \mathrm{C}\right)$ & Normal (54\%) & OK & OK & 140 & -89.941667 & 41.416667 \\
\hline $8 / 28 / 09$ 16:45 & $16: 52$ & 5703187 & Normal & OK & Normal $\left(24^{\circ} \mathrm{C}\right)$ & Normal (54\%) & OK & OK & 140 & -90.050000 & 41.413333 \\
\hline $8 / 28 / 09$ 16:50 & $16: 52$ & 5703187 & Normal & OK & Normal $\left(24^{\circ} \mathrm{C}\right)$ & Normal (54\%) & OK & OK & 140 & -90.157222 & 41.430000 \\
\hline 8/28/09 16:55 & $16: 58$ & 5703187 & Normal & OK & Normal $\left(24^{\circ} \mathrm{C}\right)$ & Normal (54\%) & OK & OK & 140 & -90.267500 & 41.440556 \\
\hline $8 / 28 / 09$ 17:00 & $17: 09$ & 5703187 & Normal & OK & Normal $\left(24^{\circ} \mathrm{C}\right)$ & Normal (54\%) & OK & OK & 140 & -90.338333 & 41.476944 \\
\hline $8 / 28 / 09$ 17:05 & $17: 09$ & 5703187 & Normal & OK & Normal $\left(24^{\circ} \mathrm{C}\right)$ & Normal (54\%) & OK & OK & 140 & -90.334167 & $41.48638 \mathrm{~s}$ \\
\hline 8/28/09 17:11 & $17: 15$ & 5703187 & Normal & OK & Normal $\left(24^{\circ} \mathrm{C}\right)$ & Normal (54\%) & OK & OK & 140 & -90.334167 & $41.48638 \mathrm{~s}$ \\
\hline 8/28/09 17:15 & $17: 20$ & 5703187 & Normal & OK & Normal $\left(24^{\circ} \mathrm{C}\right)$ & Normal (54\%) & OK & OK & 140 & -90.334167 & $41.48638 \mathrm{~s}$ \\
\hline $8 / 28 / 09$ 17:20 & $17: 26$ & 5703187 & Normal & OK & Normal $\left(24^{\circ} \mathrm{C}\right)$ & Normal (54\%) & OK & OK & 140 & -90.334167 & $41.48638 \mathrm{~s}$ \\
\hline $8 / 28 / 09$ 17:25 & $17: 31$ & 5703187 & Normal & OK & Normal $\left(24^{\circ} \mathrm{C}\right)$ & Normal (54\%) & OK & OK & 140 & -90.340556 & $41.51138 \mathrm{~s}$ \\
\hline 8/28/09 17:30 & $17: 37$ & 5703187 & Normal & OK & Normal $\left(24^{\circ} \mathrm{C}\right)$ & Normal (54\%) & OK & OK & 140 & -90.234167 & 41.562500 \\
\hline $8 / 28 / 09$ 17:35 & $17: 42$ & 5703187 & Normal & OK & Normal $\left(24^{\circ} \mathrm{C}\right)$ & Normal (54\%) & OK & OK & 140 & -90.209167 & 41.581111 \\
\hline $8 / 28 / 09$ 17:40 & $17: 42$ & 5703187 & Normal & OK & Normal $\left(24^{\circ} \mathrm{C}\right)$ & Normal (54\%) & OK & OK & 140 & -90.163333 & 41.642500 \\
\hline 8/28/09 17:45 & $17: 48$ & 5703187 & Normal & OK & Normal $\left(24^{\circ} \mathrm{C}\right)$ & Normal (54\%) & OK & OK & 140 & -90.080000 & 41.690556 \\
\hline $8 / 28 / 09$ 17:50 & $17: 53$ & 5703187 & Normal & OK & Normal $\left(24^{\circ} \mathrm{C}\right)$ & Normal (54\%) & OK & OK & 140 & -89.983333 & 41.718611 \\
\hline $8 / 28 / 09$ 17:55 & $17: 59$ & 5703187 & Normal & OK & Normal $\left(26^{\circ} \mathrm{C}\right)$ & Normal (53\%) & OK & OK & 140 & -89.876944 & 41.744722 \\
\hline $8 / 28 / 09$ 18:00 & $18: 04$ & 5703187 & Normal & OK & Normal $\left(26^{\circ} \mathrm{C}\right)$ & Normal (53\%) & OK & OK & 140 & -89.775000 & 41.757222 \\
\hline 8/28/09 18:05 & $18: 10$ & 5703187 & Normal & OK & Normal $\left(26^{\circ} \mathrm{C}\right)$ & Normal $(53 \%)$ & OK & OK & 140 & -89.689444 & 41.750833 \\
\hline $8 / 28 / 09$ 18:10 & $18: 15$ & 5703187 & Normal & OK & Normal $\left(26^{\circ} \mathrm{C}\right)$ & Normal (53\%) & OK & OK & 140 & -89.689444 & 41.762500 \\
\hline 8/28/09 18:15 & $18: 21$ & 5703187 & Normal & OK & Normal $\left(26^{\circ} \mathrm{C}\right)$ & Normal (53\%) & OK & OK & 140 & -89.678056 & 41.750833 \\
\hline 8/28/09 18:20 & $18: 26$ & 5703187 & Normal & OK & Normal $\left(26^{\circ} \mathrm{C}\right)$ & Normal (53\%) & OK & OK & 140 & -89.635278 & 41.759167 \\
\hline 8/28/09 18:25 & $18: 32$ & 5703187 & Normal & OK & Normal $\left(26^{\circ} \mathrm{C}\right)$ & Normal (53\%) & OK & OK & 140 & -89.532222 & 41.789444 \\
\hline $8 / 28 / 09$ 18:30 & $18: 38$ & 5703187 & Normal & OK & Normal $\left(26^{\circ} \mathrm{C}\right)$ & Normal (53\%) & OK & OK & 140 & -89.432222 & 41.822778 \\
\hline $8 / 28 / 09$ 18:35 & $18: 38$ & 5703187 & Normal & OK & Normal $\left(26^{\circ} \mathrm{C}\right)$ & Normal (53\%) & OK & OK & 140 & -89.336389 & 41.822778 \\
\hline 8/28/09 18:40 & $18: 43$ & 5703187 & Normal & OK & Normal $\left(26^{\circ} \mathrm{C}\right)$ & Normal (53\%) & OK & OK & 140 & -89.231111 & 41.850833 \\
\hline 8/28/09 18:45 & $18: 48$ & 5703187 & Normal & OK & Normal $\left(27^{\circ} \mathrm{C}\right)$ & Normal (51\%) & OK & OK & 140 & -89.125000 & 41.883333 \\
\hline 8/28/09 18:50 & $18: 54$ & 5703187 & Normal & OK & Normal $\left(27^{\circ} \mathrm{C}\right)$ & Normal (51\%) & OK & OK & 140 & -89.025000 & 41.904167 \\
\hline 8/28/09 18:55 & $19: 00$ & 5703187 & Normal & OK & Normal $\left(27^{\circ} \mathrm{C}\right)$ & Normal (51\%) & OK & OK & 140 & -88.913333 & 41.900000 \\
\hline 8/28/09 19:00 & 19:05 & 5703187 & Normal & $\mathrm{OK}$ & Normal $\left(27^{\circ} \mathrm{C}\right)$ & Normal (51\%) & $\mathrm{OK}$ & OK & 140 & -88.814444 & 41.900000 \\
\hline 8/28/09 19:05 & $19: 11$ & 5703187 & Normal & OK & Normal $\left(27^{\circ} \mathrm{C}\right)$ & Normal (51\%) & OK & OK & 140 & -88.739444 & 41.900000 \\
\hline 8/28/09 19:10 & $19: 16$ & 5703187 & Normal & OK & Normal $\left(27^{\circ} \mathrm{C}\right)$ & Normal $(51 \%)$ & OK & OK & 140 & -88.739444 & 41.900000 \\
\hline 8/28/09 19:15 & $19: 22$ & 5703187 & Normal & OK & Normal $\left(27^{\circ} \mathrm{C}\right)$ & Normal (50\%) & OK & OK & 140 & -88.737500 & 41.900000 \\
\hline 8/28/09 19:20 & $19: 27$ & 5703187 & Normal & OK & Normal $\left(27^{\circ} \mathrm{C}\right)$ & Normal $(50 \%)$ & OK & OK & 140 & -88.737500 & 41.900000 \\
\hline 8/28/09 19:25 & $19: 33$ & 5703187 & Normal & OK & Normal $\left(27^{\circ} \mathrm{C}\right)$ & Normal (50\%) & OK & OK & 140 & -88.639444 & 41.894722 \\
\hline 8/28/09 19:30 & $19: 38$ & 5703187 & Normal & OK & Normal $\left(27^{\circ} \mathrm{C}\right)$ & Normal (50\%) & OK & OK & 140 & -88.538333 & 41.859167 \\
\hline 8/28/09 19:35 & $19: 38$ & 5703187 & Normal & OK & Normal $\left(27^{\circ} \mathrm{C}\right)$ & Normal $(50 \%)$ & OK & OK & 140 & -88.448889 & 41.812500 \\
\hline $8 / 28 / 09$ 19:40 & $19: 44$ & 5703187 & Normal & OK & Normal $\left(27^{\circ} \mathrm{C}\right)$ & Normal $(50 \%)$ & OK & OK & 140 & -88.347778 & 41.794722 \\
\hline 8/28/09 19:45 & $19: 49$ & 5703187 & Normal & OK & Normal $\left(27^{\circ} \mathrm{C}\right)$ & Normal $(50 \%)$ & OK & OK & 140 & -88.261389 & 41.800000 \\
\hline 8/28/09 19:50 & $19: 55$ & 5703187 & Normal & OK & Normal $\left(27^{\circ} \mathrm{C}\right)$ & Normal $(50 \%)$ & OK & OK & 140 & -88.156111 & 41.806111 \\
\hline $8 / 28 / 09$ 19:55 & $20: 00$ & 5703187 & Normal & OK & Normal $\left(27^{\circ} \mathrm{C}\right)$ & Normal (50\%) & OK & OK & 140 & -88.062500 & 41.810278 \\
\hline $8 / 28 / 0920: 00$ & $20: 06$ & 5703187 & Normal & OK & Normal $\left(27^{\circ} \mathrm{C}\right)$ & Normal $(50 \%)$ & OK & OK & 140 & -88.054167 & 41.807222 \\
\hline 8/28/09 20:05 & $20: 11$ & 5703187 & Normal & OK & Normal $\left(27^{\circ} \mathrm{C}\right)$ & Normal (51\%) & OK & OK & 140 & -88.054167 & 41.781111 \\
\hline $8 / 28 / 09$ 20:10 & $20: 22$ & 5703187 & Normal & $\mathrm{OK}$ & Normal $\left(27^{\circ} \mathrm{C}\right)$ & Normal (51\%) & OK & OK & 140 & -88.035278 & 41.732222 \\
\hline $8 / 28 / 0913: 40$ & $13: 44$ & 5703188 & Normal & $\mathrm{OK}$ & Normal $\left(23^{\circ} \mathrm{C}\right)$ & Normal $(55 \%)$ & OK & OK & 141 & -87.971667 & 41.732222 \\
\hline $8 / 28 / 09$ 13:45 & $13: 49$ & 5703188 & Normal & OK & Normal $\left(23^{\circ} \mathrm{C}\right)$ & Normal (55\%) & OK & OK & 141 & -87.971667 & 41.732222 \\
\hline 8/28/09 13:50 & $14: 00$ & 5703188 & Normal & OK & Normal $\left(23^{\circ} \mathrm{C}\right)$ & Normal (55\%) & OK & OK & 141 & -87.971667 & 41.732222 \\
\hline 8/28/09 13:55 & $14: 00$ & 5703188 & Normal & OK & Normal $\left(23^{\circ} \mathrm{C}\right)$ & Normal (55\%) & OK & OK & 141 & -87.971667 & 41.732222 \\
\hline $8 / 28 / 09$ 14:00 & $14: 06$ & 5703188 & Normal & OK & Normal $\left(23^{\circ} \mathrm{C}\right)$ & Normal (55\%) & OK & OK & 141 & -88.068611 & 41.684167 \\
\hline
\end{tabular}




\begin{tabular}{|c|c|c|c|c|c|c|c|c|c|c|c|}
\hline Event Time & at QC Hub & Tag ID & Tag Status & Seal & Temperature & Humidity & Shock & Battery & RSSI & Longitude & Latitude \\
\hline $8 / 28 / 09$ 14:05 & $14: 11$ & 5703188 & Normal & OK & Normal $\left(23^{\circ} \mathrm{C}\right)$ & Normal (55\%) & OK & OK & 141 & -88.154167 & 41.640556 \\
\hline $8 / 28 / 09$ 14:10 & $14: 17$ & 5703188 & Normal & OK & Normal $\left(23^{\circ} \mathrm{C}\right)$ & Normal (55\%) & OK & OK & 141 & -88.181111 & 41.568611 \\
\hline 8/28/09 14:15 & $14: 22$ & 5703188 & Normal & OK & Normal $\left(23^{\circ} \mathrm{C}\right)$ & Normal (55\%) & OK & OK & 141 & -88.197778 & 41.493611 \\
\hline $8 / 28 / 0914: 20$ & $14: 22$ & 5703188 & Normal & OK & Normal $\left(23^{\circ} \mathrm{C}\right)$ & Normal (55\%) & OK & OK & 141 & -88.280000 & 41.462500 \\
\hline $8 / 28 / 0914: 25$ & $14: 28$ & 5703188 & Normal & OK & Normal $\left(23^{\circ} \mathrm{C}\right)$ & Normal (55\%) & OK & $\mathrm{OK}$ & 141 & -88.371667 & 41.417500 \\
\hline $8 / 28 / 09$ 14:32 & $14: 34$ & 5703188 & Normal & OK & Normal $\left(23^{\circ} \mathrm{C}\right)$ & Normal (55\%) & OK & OK & 141 & -88.422778 & 41.392500 \\
\hline 8/28/09 14:35 & $14: 39$ & 5703188 & Normal & OK & Normal $\left(23^{\circ} \mathrm{C}\right)$ & Normal (55\%) & OK & OK & 141 & -88.422778 & 41.392500 \\
\hline $8 / 28 / 09$ 14:40 & $14: 44$ & 5703188 & Normal & OK & Normal $\left(23^{\circ} \mathrm{C}\right)$ & Normal $(55 \%)$ & OK & OK & 141 & -88.422778 & 41.392500 \\
\hline $8 / 28 / 0914: 45$ & $14: 50$ & 5703188 & Normal & OK & Normal $\left(23^{\circ} \mathrm{C}\right)$ & Normal (55\%) & OK & $\mathrm{OK}$ & 141 & -88.461389 & 41.378056 \\
\hline $8 / 28 / 0914: 50$ & $14: 56$ & 5703188 & Normal & OK & Normal $\left(23^{\circ} \mathrm{C}\right)$ & Normal (55\%) & OK & $\mathrm{OK}$ & 141 & -88.575000 & 41.376944 \\
\hline $8 / 28 / 0914: 55$ & 15:01 & 5703188 & Normal & OK & Normal $\left(23^{\circ} \mathrm{C}\right)$ & Normal (53\%) & OK & OK & 141 & -88.717500 & 41.375833 \\
\hline $8 / 28 / 09$ 15:00 & 15:07 & 5703188 & Normal & OK & Normal $\left(23^{\circ} \mathrm{C}\right)$ & Normal (53\%) & OK & OK & 141 & -88.792500 & 41.376944 \\
\hline 8/28/09 15:05 & $15: 12$ & 5703188 & Normal & OK & Normal $\left(23^{\circ} \mathrm{C}\right)$ & Normal (53\%) & OK & OK & 141 & -88.901944 & 41.368611 \\
\hline 8/28/09 15:10 & $15: 12$ & 5703188 & Normal & OK & Normal $\left(23^{\circ} \mathrm{C}\right)$ & Normal (53\%) & OK & OK & 141 & -89.014444 & 41.367500 \\
\hline 8/28/09 15:15 & $15: 18$ & 5703188 & Normal & OK & Normal $\left(23^{\circ} \mathrm{C}\right)$ & Normal (53\%) & OK & OK & 141 & -89.058333 & 41.413333 \\
\hline $8 / 28 / 09$ 15:20 & $15: 23$ & 5703188 & Normal & OK & Normal $\left(23^{\circ} \mathrm{C}\right)$ & Normal (53\%) & OK & OK & 141 & -89.050833 & 41.460278 \\
\hline 8/28/09 15:25 & $15: 29$ & 5703188 & Normal & OK & Normal $\left(23^{\circ} \mathrm{C}\right)$ & Normal (53\%) & OK & OK & 141 & -89.058333 & 41.375833 \\
\hline 8/28/09 15:30 & $15: 35$ & 5703188 & Normal & OK & Normal $\left(23^{\circ} \mathrm{C}\right)$ & Normal (53\%) & OK & OK & 141 & -89.125833 & 41.368611 \\
\hline 8/28/09 15:35 & $15: 40$ & 5703188 & Normal & OK & Normal $\left(23^{\circ} \mathrm{C}\right)$ & Normal (53\%) & OK & $\mathrm{OK}$ & 141 & -89.125833 & 41.368611 \\
\hline 8/28/09 15:40 & $15: 46$ & 5703188 & Normal & OK & Normal $\left(23^{\circ} \mathrm{C}\right)$ & Normal (53\%) & OK & OK & 141 & -89.125833 & 41.368611 \\
\hline 8/28/09 15:45 & $15: 51$ & 5703188 & Normal & OK & Normal $\left(23^{\circ} \mathrm{C}\right)$ & Normal (53\%) & OK & $\mathrm{OK}$ & 141 & -89.200000 & 41.364444 \\
\hline $8 / 28 / 09$ 15:50 & $15: 57$ & 5703188 & Normal & OK & Normal $\left(23^{\circ} \mathrm{C}\right)$ & Normal (53\%) & OK & $\mathrm{OK}$ & 141 & -89.240556 & 41.371667 \\
\hline $8 / 28 / 0916: 00$ & $16: 03$ & 5703188 & Normal & OK & Normal $\left(23^{\circ} \mathrm{C}\right)$ & Normal (53\%) & OK & OK & 141 & -89.458333 & 41.400833 \\
\hline $8 / 28 / 09$ 16:05 & $16: 13$ & 5703188 & Normal & OK & Normal $\left(23^{\circ} \mathrm{C}\right)$ & Normal (53\%) & OK & OK & 141 & -89.558333 & 41.393611 \\
\hline 8/28/09 16:11 & $16: 14$ & 5703188 & Normal & OK & Normal $\left(23^{\circ} \mathrm{C}\right)$ & Normal (53\%) & OK & OK & 141 & -89.558333 & 41.393611 \\
\hline 8/28/09 16:15 & $16: 19$ & 5703188 & Normal & OK & Normal $\left(23^{\circ} \mathrm{C}\right)$ & Normal (53\%) & OK & OK & 141 & -89.558333 & 41.393611 \\
\hline $8 / 28 / 0916: 20$ & $16: 25$ & 5703188 & Normal & OK & Normal $\left(23^{\circ} \mathrm{C}\right)$ & Normal (53\%) & OK & OK & 141 & -89.558333 & 41.393611 \\
\hline $8 / 28 / 0916: 25$ & $16: 30$ & 5703188 & Normal & OK & Normal $\left(23^{\circ} \mathrm{C}\right)$ & Normal (53\%) & OK & OK & 141 & -89.623889 & 41.394722 \\
\hline $8 / 28 / 0916: 30$ & $16: 36$ & 5703188 & Normal & OK & Normal $\left(23^{\circ} \mathrm{C}\right)$ & Normal (53\%) & OK & OK & 141 & -89.725833 & 41.387500 \\
\hline $8 / 28 / 09$ 16:35 & $16: 41$ & 5703188 & Normal & OK & Normal $\left(23^{\circ} \mathrm{C}\right)$ & Normal (53\%) & OK & OK & 141 & -89.834167 & 41.388333 \\
\hline $8 / 28 / 09$ 16:40 & $16: 47$ & 5703188 & Normal & OK & Normal $\left(23^{\circ} \mathrm{C}\right)$ & Normal (53\%) & OK & OK & 141 & -89.941667 & 41.416667 \\
\hline $8 / 28 / 0916: 45$ & $16: 52$ & 5703188 & Normal & OK & Normal $\left(23^{\circ} \mathrm{C}\right)$ & Normal (53\%) & OK & OK & 141 & -90.050000 & 41.413333 \\
\hline $8 / 28 / 09$ 16:50 & $16: 52$ & 5703188 & Normal & OK & Normal $\left(23^{\circ} \mathrm{C}\right)$ & Normal (53\%) & OK & OK & 141 & -90.157222 & 41.430000 \\
\hline $8 / 28 / 09$ 16:55 & $16: 58$ & 5703188 & Normal & OK & Normal $\left(23^{\circ} \mathrm{C}\right)$ & Normal (53\%) & OK & OK & 141 & -90.267500 & 41.440556 \\
\hline 8/28/09 17:00 & $17: 09$ & 5703188 & Normal & OK & Normal $\left(23^{\circ} \mathrm{C}\right)$ & Normal (53\%) & OK & OK & 141 & -90.338333 & 41.476944 \\
\hline $8 / 28 / 09$ 17:05 & $17: 09$ & 5703188 & Normal & OK & Normal $\left(23^{\circ} \mathrm{C}\right)$ & Normal (53\%) & OK & OK & 141 & -90.334167 & 41.486389 \\
\hline $8 / 28 / 09$ 17:11 & $17: 15$ & 5703188 & Normal & OK & Normal $\left(23^{\circ} \mathrm{C}\right)$ & Normal (53\%) & OK & OK & 141 & -90.334167 & 41.486389 \\
\hline $8 / 28 / 09$ 17:15 & $17: 20$ & 5703188 & Normal & OK & Normal $\left(23^{\circ} \mathrm{C}\right)$ & Normal (53\%) & OK & OK & 141 & -90.334167 & 41.486389 \\
\hline $8 / 28 / 09$ 17:20 & $17: 26$ & 5703188 & Normal & OK & Normal $\left(23^{\circ} \mathrm{C}\right)$ & Normal (53\%) & OK & OK & 141 & -90.334167 & 41.486389 \\
\hline 8/28/09 17:25 & $17: 31$ & 5703188 & Normal & OK & Normal $\left(23^{\circ} \mathrm{C}\right)$ & Normal (53\%) & OK & OK & 141 & -90.340556 & 41.511389 \\
\hline 8/28/09 17:30 & $17: 37$ & 5703188 & Normal & OK & Normal $\left(23^{\circ} \mathrm{C}\right)$ & Normal (53\%) & OK & $\mathrm{OK}$ & 141 & -90.234167 & 41.562500 \\
\hline 8/28/09 17:35 & $17: 42$ & 5703188 & Normal & OK & Normal $\left(23^{\circ} \mathrm{C}\right)$ & Normal (53\%) & OK & $\mathrm{OK}$ & 141 & -90.209167 & 41.581111 \\
\hline 8/28/09 17:40 & $17: 42$ & 5703188 & Normal & OK & Normal $\left(23^{\circ} \mathrm{C}\right)$ & Normal (53\%) & OK & OK & 141 & -90.163333 & 41.642500 \\
\hline 8/28/09 17:45 & $17: 48$ & 5703188 & Normal & OK & Normal $\left(23^{\circ} \mathrm{C}\right)$ & Normal (53\%) & OK & OK & 141 & -90.080000 & 41.690556 \\
\hline $8 / 28 / 09$ 17:50 & $17: 53$ & 5703188 & Normal & OK & $\operatorname{Normal}\left(23^{\circ} \mathrm{C}\right)$ & Normal (53\%) & OK & OK & 141 & -89.983333 & 41.718611 \\
\hline $8 / 28 / 09$ 17:55 & $17: 59$ & 5703188 & Normal & OK & Normal $\left(26^{\circ} \mathrm{C}\right)$ & Normal (54\%) & OK & OK & 141 & -89.876944 & 41.744722 \\
\hline 8/28/09 18:00 & $18: 04$ & 5703188 & Normal & OK & Normal $\left(26^{\circ} \mathrm{C}\right)$ & Normal (54\%) & OK & $\mathrm{OK}$ & 141 & -89.775000 & 41.757222 \\
\hline 8/28/09 18:05 & $18: 10$ & 5703188 & Normal & OK & Normal $\left(26^{\circ} \mathrm{C}\right)$ & Normal (54\%) & OK & OK & 141 & -89.689444 & 41.750833 \\
\hline $8 / 28 / 09$ 18:10 & $18: 15$ & 5703188 & Normal & OK & Normal $\left(26^{\circ} \mathrm{C}\right)$ & Normal (54\%) & OK & OK & 141 & -89.689444 & 41.762500 \\
\hline $8 / 28 / 09$ 18:15 & $18: 21$ & 5703188 & Normal & OK & Normal $\left(26^{\circ} \mathrm{C}\right)$ & Normal (54\%) & OK & OK & 141 & -89.678056 & 41.750833 \\
\hline $8 / 28 / 0918: 20$ & $18: 26$ & 5703188 & Normal & OK & Normal $\left(26^{\circ} \mathrm{C}\right)$ & Normal (54\%) & OK & OK & 141 & -89.635278 & 41.759167 \\
\hline $8 / 28 / 0918: 25$ & $18: 32$ & 5703188 & Normal & OK & Normal $\left(26^{\circ} \mathrm{C}\right)$ & Normal (54\%) & OK & OK & 141 & -89.532222 & 41.789444 \\
\hline $8 / 28 / 0918: 30$ & $18: 38$ & 5703188 & Normal & OK & Normal $\left(26^{\circ} \mathrm{C}\right)$ & Normal (54\%) & OK & OK & 141 & -89.432222 & 41.822778 \\
\hline $8 / 28 / 09$ 18:35 & $18: 38$ & 5703188 & Normal & OK & Normal $\left(26^{\circ} \mathrm{C}\right)$ & Normal (54\%) & OK & OK & 141 & -89.336389 & 41.822778 \\
\hline $8 / 28 / 0918: 40$ & $18: 43$ & 5703188 & Normal & OK & Normal $\left(26^{\circ} \mathrm{C}\right)$ & Normal (54\%) & OK & OK & 141 & -89.231111 & 41.850833 \\
\hline $8 / 28 / 0918: 45$ & $18: 48$ & 5703188 & Normal & OK & Normal $\left(26^{\circ} \mathrm{C}\right)$ & Normal $(52 \%)$ & OK & OK & 141 & -89.125000 & 41.883333 \\
\hline 8/28/09 18:50 & $18: 54$ & 5703188 & Normal & OK & Normal $\left(26^{\circ} \mathrm{C}\right)$ & Normal (52\%) & OK & $\mathrm{OK}$ & 141 & -89.025000 & 41.904167 \\
\hline 8/28/09 18:55 & $19: 00$ & 5703188 & Normal & OK & Normal $\left(26^{\circ} \mathrm{C}\right)$ & Normal (52\%) & OK & OK & 141 & -88.913333 & 41.900000 \\
\hline 8/28/09 19:00 & 19:05 & 5703188 & Normal & OK & Normal $\left(26^{\circ} \mathrm{C}\right)$ & Normal (52\%) & OK & OK & 141 & -88.814444 & 41.900000 \\
\hline 8/28/09 19:05 & $19: 11$ & 5703188 & Normal & OK & Normal $\left(26^{\circ} \mathrm{C}\right)$ & Normal (52\%) & OK & OK & 141 & -88.739444 & 41.900000 \\
\hline $8 / 28 / 09$ 19:10 & $19: 16$ & 5703188 & Normal & OK & Normal $\left(26^{\circ} \mathrm{C}\right)$ & Normal $(52 \%)$ & OK & OK & 141 & -88.739444 & 41.900000 \\
\hline $8 / 28 / 09$ 19:15 & $19: 22$ & 5703188 & Normal & OK & Normal $\left(26^{\circ} \mathrm{C}\right)$ & Normal $(51 \%)$ & OK & OK & 141 & -88.737500 & 41.900000 \\
\hline $8 / 28 / 09$ 19:20 & $19: 27$ & 5703188 & Normal & OK & Normal $\left(26^{\circ} \mathrm{C}\right)$ & Normal $(51 \%)$ & OK & OK & 141 & -88.737500 & 41.900000 \\
\hline $8 / 28 / 09$ 19:25 & $19: 33$ & 5703188 & Normal & OK & Normal $\left(26^{\circ} \mathrm{C}\right)$ & Normal (51\%) & OK & OK & 141 & -88.639444 & 41.894722 \\
\hline $8 / 28 / 09$ 19:30 & $19: 38$ & 5703188 & Normal & OK & Normal $\left(26^{\circ} \mathrm{C}\right)$ & Normal (51\%) & OK & OK & 141 & -88.538333 & 41.859167 \\
\hline 8/28/09 19:35 & 19:38 & 5703188 & Normal & OK & Normal $\left(26^{\circ} \mathrm{C}\right)$ & Normal (51\%) & OK & OK & 141 & -88.448889 & 41.812500 \\
\hline 8/28/09 19:40 & $19: 44$ & 5703188 & Normal & OK & Normal $\left(26^{\circ} \mathrm{C}\right)$ & Normal (51\%) & OK & $\mathrm{OK}$ & 141 & -88.347778 & 41.794722 \\
\hline 8/28/09 19:45 & $19: 49$ & 5703188 & Normal & OK & Normal $\left(26^{\circ} \mathrm{C}\right)$ & Normal (51\%) & OK & OK & 141 & -88.261389 & 41.800000 \\
\hline $8 / 28 / 09$ 19:50 & $19: 55$ & 5703188 & Normal & OK & Normal $\left(26^{\circ} \mathrm{C}\right)$ & Normal (51\%) & OK & OK & 141 & -88.156111 & 41.806111 \\
\hline 8/28/09 19:55 & $20: 00$ & 5703188 & Normal & OK & Normal $\left(26^{\circ} \mathrm{C}\right)$ & Normal (51\%) & OK & OK & 141 & -88.062500 & 41.810278 \\
\hline 8/28/09 20:00 & $20: 06$ & 5703188 & Normal & OK & Normal $\left(26^{\circ} \mathrm{C}\right)$ & Normal (51\%) & OK & OK & 141 & -88.054167 & 41.807222 \\
\hline 8/28/09 20:05 & $20: 11$ & 5703188 & Normal & OK & Normal $\left(26^{\circ} \mathrm{C}\right)$ & Normal (52\%) & OK & $\mathrm{OK}$ & 141 & -88.054167 & 41.781111 \\
\hline 8/28/09 20:10 & $20: 22$ & 5703188 & Normal & OK & Normal $\left(26^{\circ} \mathrm{C}\right)$ & Normal (52\%) & OK & OK & 141 & -88.035278 & 41.732222 \\
\hline $8 / 28 / 0913: 40$ & $13: 44$ & 5703191 & Normal & OK & Normal $\left(23^{\circ} \mathrm{C}\right)$ & Normal (52\%) & $\overline{O K}$ & $\overline{\mathrm{OK}}$ & 142 & -87.971667 & 41.732222 \\
\hline $8 / 28 / 09$ 13:45 & $13: 49$ & 5703191 & Normal & OK & Normal $\left(23^{\circ} \mathrm{C}\right)$ & Normal (52\%) & OK & OK & 142 & -87.971667 & 41.732222 \\
\hline $8 / 28 / 0913: 50$ & $14: 00$ & 5703191 & Normal & OK & Normal $\left(23^{\circ} \mathrm{C}\right)$ & Normal (52\%) & OK & $\mathrm{OK}$ & 142 & -87.971667 & 41.732222 \\
\hline $8 / 28 / 0913: 55$ & $14: 00$ & 5703191 & Normal & OK & Normal $\left(23^{\circ} \mathrm{C}\right)$ & Normal $(52 \%)$ & OK & OK & 142 & -87.971667 & 41.732222 \\
\hline $8 / 28 / 0914: 00$ & $14: 06$ & 5703191 & Normal & OK & Normal $\left(23^{\circ} \mathrm{C}\right)$ & Normal $(52 \%)$ & OK & OK & 142 & -88.068611 & 41.684167 \\
\hline 8/28/09 14:05 & $14: 11$ & 5703191 & Normal & OK & $\operatorname{Normal}\left(23^{\circ} \mathrm{C}\right)$ & Normal (52\%) & OK & OK & 142 & -88.154167 & 41.640556 \\
\hline $8 / 28 / 09$ 14:10 & $14: 17$ & 5703191 & Normal & OK & Normal $\left(23^{\circ} \mathrm{C}\right)$ & Normal (52\%) & OK & OK & 142 & -88.181111 & 41.568611 \\
\hline $8 / 28 / 09$ 14:15 & $14: 22$ & 5703191 & Normal & OK & Normal $\left(23^{\circ} \mathrm{C}\right)$ & Normal (52\%) & OK & $\mathrm{OK}$ & 142 & -88.197778 & 41.493611 \\
\hline
\end{tabular}




\begin{tabular}{|c|c|c|c|c|c|c|c|c|c|c|c|}
\hline Event Time & at $Q C$ Hub & Tag ID & Tag Status & Seal & Temperature & Humidity & Shock & Battery & RSSI & Longitude & Latitude \\
\hline $8 / 28 / 0914: 20$ & $14: 22$ & 5703191 & Normal & OK & Normal $\left(23^{\circ} \mathrm{C}\right)$ & Normal $(52 \%)$ & OK & OK & 142 & -88.280000 & 41.462500 \\
\hline $8 / 28 / 09$ 14:25 & $14: 28$ & 5703191 & Normal & OK & Normal $\left(23^{\circ} \mathrm{C}\right)$ & Normal (52\%) & OK & OK & 142 & -88.371667 & 41.417500 \\
\hline $8 / 28 / 0914: 32$ & $14: 39$ & 5703191 & Normal & OK & Normal $\left(23^{\circ} \mathrm{C}\right)$ & Normal (52\%) & OK & OK & 142 & -88.422778 & 41.392500 \\
\hline $8 / 28 / 0914: 35$ & $14: 39$ & 5703191 & Normal & OK & Normal $\left(23^{\circ} \mathrm{C}\right)$ & Normal $(52 \%)$ & OK & OK & 142 & -88.422778 & 41.392500 \\
\hline $8 / 28 / 0914: 40$ & $14: 44$ & 5703191 & Normal & OK & Normal $\left(23^{\circ} \mathrm{C}\right)$ & Normal (52\%) & $\mathrm{OK}$ & OK & 142 & -88.422778 & 41.392500 \\
\hline $8 / 28 / 09$ 14:45 & $14: 50$ & 5703191 & Normal & OK & Normal $\left(23^{\circ} \mathrm{C}\right)$ & Normal (52\%) & OK & OK & 142 & -88.461389 & 41.378056 \\
\hline $8 / 28 / 09$ 14:50 & $14: 56$ & 5703191 & Normal & OK & Normal $\left(23^{\circ} \mathrm{C}\right)$ & Normal (52\%) & $\mathrm{OK}$ & OK & 142 & -88.575000 & 41.376944 \\
\hline $8 / 28 / 0914: 55$ & 15:01 & 5703191 & Normal & OK & Normal $\left(23^{\circ} \mathrm{C}\right)$ & Normal (49\%) & OK & OK & 142 & -88.717500 & 41.375833 \\
\hline $8 / 28 / 09$ 15:00 & $15: 07$ & 5703191 & Normal & OK & Normal $\left(23^{\circ} \mathrm{C}\right)$ & Normal (49\%) & $\mathrm{OK}$ & OK & 142 & -88.792500 & 41.376944 \\
\hline $8 / 28 / 09$ 15:05 & $15: 12$ & 5703191 & Normal & OK & Normal $\left(23^{\circ} \mathrm{C}\right)$ & Normal (49\%) & OK & OK & 142 & -88.901944 & 41.368611 \\
\hline $8 / 28 / 09$ 15:10 & $15: 12$ & 5703191 & Normal & OK & Normal $\left(23^{\circ} \mathrm{C}\right)$ & Normal (49\%) & OK & OK & 142 & -89.014444 & 41.367500 \\
\hline $8 / 28 / 09$ 15:15 & $15: 18$ & 5703191 & Normal & OK & Normal $\left(23^{\circ} \mathrm{C}\right)$ & Normal (49\%) & OK & OK & 142 & -89.058333 & 41.413333 \\
\hline $8 / 28 / 0915: 20$ & $15: 23$ & 5703191 & Normal & OK & Normal $\left(23^{\circ} \mathrm{C}\right)$ & Normal $(49 \%)$ & OK & OK & 142 & -89.050833 & 41.460278 \\
\hline $8 / 28 / 09$ 15:25 & $15: 29$ & 5703191 & Normal & OK & Normal $\left(23^{\circ} \mathrm{C}\right)$ & Normal (49\%) & OK & OK & 142 & -89.058333 & 41.375833 \\
\hline $8 / 28 / 09$ 15:30 & $15: 35$ & 5703191 & Normal & OK & Normal $\left(23^{\circ} \mathrm{C}\right)$ & Normal $(49 \%)$ & OK & OK & 142 & -89.125833 & 41.368611 \\
\hline $8 / 28 / 09$ 15:32 & $15: 35$ & 5703191 & Alarm & OK & Normal $\left(23^{\circ} \mathrm{C}\right)$ & Normal (49\%) & Shocked & OK & 142 & -89.125833 & 41.368611 \\
\hline $8 / 28 / 0915: 35$ & $15: 40$ & 5703191 & Normal & OK & Normal $\left(23^{\circ} \mathrm{C}\right)$ & Normal (48\%) & OK & OK & 142 & -89.125833 & 41.368611 \\
\hline $8 / 28 / 0915: 40$ & $15: 46$ & 5703191 & Normal & OK & Normal $\left(23^{\circ} \mathrm{C}\right)$ & Normal $(48 \%)$ & OK & OK & 142 & -89.125833 & 41.368611 \\
\hline $8 / 28 / 0915: 45$ & $15: 51$ & 5703191 & Normal & OK & Normal $\left(23^{\circ} \mathrm{C}\right)$ & Normal $(48 \%)$ & OK & OK & 142 & -89.200000 & 41.364444 \\
\hline $8 / 28 / 09$ 15:50 & $15: 57$ & 5703191 & Normal & OK & Normal $\left(23^{\circ} \mathrm{C}\right)$ & Normal (48\%) & $\mathrm{OK}$ & OK & 142 & -89.240556 & 41.371667 \\
\hline $8 / 28 / 0916: 00$ & $16: 03$ & 5703191 & Normal & OK & Normal $\left(23^{\circ} \mathrm{C}\right)$ & Normal (48\%) & OK & OK & 142 & -89.458333 & 41.400833 \\
\hline $8 / 28 / 09$ 16:05 & $16: 13$ & 5703191 & Normal & OK & Normal $\left(23^{\circ} \mathrm{C}\right)$ & Normal $(48 \%)$ & OK & OK & 142 & -89.558333 & 41.393611 \\
\hline $8 / 28 / 0916: 11$ & $16: 14$ & 5703191 & Normal & OK & Normal $\left(23^{\circ} \mathrm{C}\right)$ & Normal (48\%) & OK & OK & 142 & -89.558333 & 41.393611 \\
\hline $8 / 28 / 09$ 16:15 & $16: 19$ & 5703191 & Normal & OK & Normal $\left(23^{\circ} \mathrm{C}\right)$ & Normal $(48 \%)$ & OK & OK & 142 & -89.558333 & 41.393611 \\
\hline $8 / 28 / 09$ 16:20 & $16: 25$ & 5703191 & Normal & OK & Normal $\left(23^{\circ} \mathrm{C}\right)$ & Normal (48\%) & OK & OK & 142 & -89.558333 & 41.393611 \\
\hline $8 / 28 / 09$ 16:25 & $16: 30$ & 5703191 & Normal & OK & Normal $\left(23^{\circ} \mathrm{C}\right)$ & Normal $(48 \%)$ & OK & OK & 142 & -89.623889 & 41.394722 \\
\hline $8 / 28 / 09$ 16:30 & $16: 36$ & 5703191 & Normal & OK & Normal $\left(23^{\circ} \mathrm{C}\right)$ & Normal $(48 \%)$ & OK & OK & 142 & -89.725833 & 41.387500 \\
\hline $8 / 28 / 09$ 16:35 & $16: 41$ & 5703191 & Normal & OK & Normal $\left(23^{\circ} \mathrm{C}\right)$ & Normal (48\%) & OK & OK & 142 & -89.834167 & 41.388333 \\
\hline $8 / 28 / 09$ 16:40 & $16: 47$ & 5703191 & Normal & OK & Normal $\left(23^{\circ} \mathrm{C}\right)$ & Normal ( $48 \%)$ & OK & OK & 142 & -89.941667 & 41.416667 \\
\hline $8 / 28 / 09$ 16:45 & $16: 52$ & 5703191 & Normal & OK & Normal $\left(23^{\circ} \mathrm{C}\right)$ & Normal $(48 \%)$ & OK & OK & 142 & -90.050000 & 41.413333 \\
\hline $8 / 28 / 09$ 16:50 & $16: 52$ & 5703191 & Normal & OK & Normal $\left(23^{\circ} \mathrm{C}\right)$ & Normal $(48 \%)$ & OK & OK & 142 & -90.157222 & 41.430000 \\
\hline $8 / 28 / 09$ 16:55 & $16: 58$ & 5703191 & Normal & OK & Normal $\left(23^{\circ} \mathrm{C}\right)$ & Normal $(48 \%)$ & OK & OK & 142 & -90.267500 & 41.440556 \\
\hline $8 / 28 / 09$ 17:00 & $17: 09$ & 5703191 & Normal & OK & Normal $\left(23^{\circ} \mathrm{C}\right)$ & Normal $(48 \%)$ & OK & OK & 142 & -90.338333 & 41.476944 \\
\hline $8 / 28 / 09$ 17:05 & $17: 09$ & 5703191 & Normal & OK & Normal $\left(23^{\circ} \mathrm{C}\right)$ & Normal $(48 \%)$ & OK & OK & 142 & -90.334167 & 41.486389 \\
\hline 8/28/09 17:11 & $17: 15$ & 5703191 & Normal & OK & Normal $\left(23^{\circ} \mathrm{C}\right)$ & Normal (48\%) & OK & OK & 142 & -90.334167 & 41.486389 \\
\hline $8 / 28 / 09$ 17:15 & $17: 20$ & 5703191 & Normal & OK & Normal $\left(23^{\circ} \mathrm{C}\right)$ & Normal $(48 \%)$ & OK & OK & 142 & -90.334167 & 41.486389 \\
\hline $8 / 28 / 0917: 20$ & $17: 26$ & 5703191 & Normal & OK & Normal $\left(23^{\circ} \mathrm{C}\right)$ & Normal $(48 \%)$ & OK & OK & 142 & -90.334167 & 41.486389 \\
\hline $8 / 28 / 09$ 17:25 & $17: 31$ & 5703191 & Normal & OK & Normal $\left(23^{\circ} \mathrm{C}\right)$ & Normal $(48 \%)$ & OK & OK & 142 & -90.340556 & 41.511389 \\
\hline $8 / 28 / 09$ 17:30 & $17: 37$ & 5703191 & Normal & OK & Normal $\left(23^{\circ} \mathrm{C}\right)$ & Normal $(48 \%)$ & OK & OK & 142 & -90.234167 & 41.562500 \\
\hline $8 / 28 / 09$ 17:35 & $17: 42$ & 5703191 & Normal & OK & Normal $\left(23^{\circ} \mathrm{C}\right)$ & Normal (48\%) & OK & OK & 142 & -90.209167 & 41.581111 \\
\hline $8 / 28 / 0917: 40$ & $17: 42$ & 5703191 & Normal & OK & Normal $\left(23^{\circ} \mathrm{C}\right)$ & Normal $(48 \%)$ & OK & OK & 142 & -90.163333 & 41.642500 \\
\hline $8 / 28 / 09$ 17:45 & $17: 48$ & 5703191 & Normal & OK & Normal $\left(23^{\circ} \mathrm{C}\right)$ & Normal (48\%) & OK & OK & 142 & -90.080000 & 41.690556 \\
\hline $8 / 28 / 09$ 17:50 & $17: 53$ & 5703191 & Normal & OK & Normal $\left(23^{\circ} \mathrm{C}\right)$ & Normal $(48 \%)$ & OK & OK & 142 & -89.983333 & 41.718611 \\
\hline $8 / 28 / 09$ 17:55 & $17: 59$ & 5703191 & Normal & OK & Normal $\left(27^{\circ} \mathrm{C}\right)$ & Normal (50\%) & OK & OK & 142 & -89.876944 & 41.744722 \\
\hline $8 / 28 / 0918: 00$ & $18: 04$ & 5703191 & Normal & OK & Normal $\left(27^{\circ} \mathrm{C}\right)$ & Normal $(50 \%)$ & OK & OK & 142 & -89.775000 & 41.757222 \\
\hline $8 / 28 / 09$ 18:05 & $18: 10$ & 5703191 & Normal & OK & Normal $\left(27^{\circ} \mathrm{C}\right)$ & Normal (50\%) & OK & OK & 142 & -89.689444 & 41.750833 \\
\hline $8 / 28 / 09$ 18:10 & $18: 15$ & 5703191 & Normal & OK & Normal $\left(27^{\circ} \mathrm{C}\right)$ & Normal (50\%) & OK & OK & 142 & -89.689444 & 41.762500 \\
\hline $8 / 28 / 09$ 18:15 & $18: 21$ & 5703191 & Normal & OK & Normal $\left(27^{\circ} \mathrm{C}\right)$ & Normal (50\%) & OK & OK & 142 & -89.678056 & 41.750833 \\
\hline $8 / 28 / 0918: 20$ & $18: 26$ & 5703191 & Normal & OK & Normal $\left(27^{\circ} \mathrm{C}\right)$ & Normal $(50 \%)$ & OK & OK & 142 & -89.635278 & 41.759167 \\
\hline $8 / 28 / 0918: 25$ & $18: 32$ & 5703191 & Normal & OK & Normal $\left(27^{\circ} \mathrm{C}\right)$ & Normal (50\%) & OK & OK & 142 & -89.532222 & 41.789444 \\
\hline $8 / 28 / 09$ 18:30 & $18: 38$ & 5703191 & Normal & OK & Normal $\left(27^{\circ} \mathrm{C}\right)$ & Normal (50\%) & OK & OK & 142 & -89.432222 & 41.822778 \\
\hline $8 / 28 / 09$ 18:35 & $18: 38$ & 5703191 & Normal & OK & Normal $\left(27^{\circ} \mathrm{C}\right)$ & Normal (50\%) & OK & OK & 142 & -89.336389 & 41.822778 \\
\hline $8 / 28 / 0918: 40$ & $18: 43$ & 5703191 & Normal & OK & Normal $\left(27^{\circ} \mathrm{C}\right)$ & Normal (50\%) & OK & OK & 142 & -89.231111 & 41.850833 \\
\hline $8 / 28 / 09$ 18:45 & $18: 48$ & 5703191 & Normal & OK & Normal $\left(27^{\circ} \mathrm{C}\right)$ & Normal $(49 \%)$ & OK & OK & 142 & -89.125000 & 41.883333 \\
\hline $8 / 28 / 09$ 18:50 & $18: 54$ & 5703191 & Normal & OK & Normal $\left(27^{\circ} \mathrm{C}\right)$ & Normal $(49 \%)$ & OK & OK & 142 & -89.025000 & 41.904167 \\
\hline $8 / 28 / 09$ 18:55 & 19:00 & 5703191 & Normal & OK & Normal $\left(27^{\circ} \mathrm{C}\right)$ & Normal (49\%) & OK & OK & 142 & -88.913333 & 41.900000 \\
\hline 8/28/09 19:00 & $19: 05$ & 5703191 & Normal & OK & Normal $\left(27^{\circ} \mathrm{C}\right)$ & Normal $(49 \%)$ & OK & OK & 142 & -88.814444 & 41.900000 \\
\hline $8 / 28 / 09$ 19:05 & $19: 11$ & 5703191 & Normal & OK & Normal $\left(27^{\circ} \mathrm{C}\right)$ & Normal $(49 \%)$ & OK & OK & 142 & -88.739444 & 41.900000 \\
\hline 8/28/09 19:10 & $19: 16$ & 5703191 & Normal & OK & Normal $\left(27^{\circ} \mathrm{C}\right)$ & Normal $(49 \%)$ & OK & OK & 142 & -88.739444 & 41.900000 \\
\hline 8/28/09 19:15 & $19: 22$ & 5703191 & Normal & OK & Normal $\left(27^{\circ} \mathrm{C}\right)$ & Normal $(48 \%)$ & OK & OK & 142 & -88.737500 & 41.900000 \\
\hline $8 / 28 / 09$ 19:20 & $19: 27$ & 5703191 & Normal & OK & Normal $\left(27^{\circ} \mathrm{C}\right)$ & Normal (48\%) & OK & OK & 142 & -88.737500 & 41.900000 \\
\hline $8 / 28 / 09$ 19:25 & $19: 33$ & 5703191 & Normal & OK & Normal $\left(27^{\circ} \mathrm{C}\right)$ & Normal $(48 \%)$ & OK & OK & 142 & -88.639444 & 41.894722 \\
\hline $8 / 28 / 09$ 19:30 & $19: 38$ & 5703191 & Normal & OK & Normal $\left(27^{\circ} \mathrm{C}\right)$ & Normal $(48 \%)$ & OK & OK & 142 & -88.538333 & 41.859167 \\
\hline $8 / 28 / 09$ 19:35 & $19: 38$ & 5703191 & Normal & OK & Normal $\left(27^{\circ} \mathrm{C}\right)$ & Normal $(48 \%)$ & OK & OK & 142 & -88.448889 & 41.812500 \\
\hline $8 / 28 / 0919: 40$ & $19: 44$ & 5703191 & Normal & OK & Normal $\left(27^{\circ} \mathrm{C}\right)$ & Normal (48\%) & OK & OK & 142 & -88.347778 & 41.794722 \\
\hline $8 / 28 / 09$ 19:45 & $19: 49$ & 5703191 & Normal & OK & Normal $\left(27^{\circ} \mathrm{C}\right)$ & Normal $(48 \%)$ & OK & OK & 142 & -88.261389 & 41.800000 \\
\hline $8 / 28 / 09$ 19:50 & $19: 55$ & 5703191 & Normal & OK & Normal $\left(27^{\circ} \mathrm{C}\right)$ & Normal $(48 \%)$ & OK & OK & 142 & -88.156111 & 41.806111 \\
\hline $8 / 28 / 09$ 19:55 & $20: 00$ & 5703191 & Normal & OK & Normal $\left(27^{\circ} \mathrm{C}\right)$ & Normal (48\%) & OK & OK & 142 & -88.062500 & 41.810278 \\
\hline $8 / 28 / 0920: 00$ & $20: 06$ & 5703191 & Normal & OK & Normal $\left(27^{\circ} \mathrm{C}\right)$ & Normal $(48 \%)$ & OK & OK & 142 & -88.054167 & 41.807222 \\
\hline 8/28/09 20:05 & $20: 11$ & 5703191 & Normal & OK & Normal $\left(27^{\circ} \mathrm{C}\right)$ & Normal (50\%) & OK & OK & 142 & -88.054167 & 41.781111 \\
\hline $8 / 28 / 0920: 10$ & $20: 22$ & 5703191 & Normal & OK & Normal $\left(27^{\circ} \mathrm{C}\right)$ & Normal $(50 \%)$ & OK & OK & 142 & -88.035278 & 41.732222 \\
\hline $8 / 28 / 0913: 40$ & $13: 44$ & 5703193 & Normal & OK & Normal $\left(23^{\circ} \mathrm{C}\right)$ & Normal (54\%) & $\mathrm{OK}$ & OK & 140 & -87.971667 & 41.732222 \\
\hline $8 / 28 / 09$ 13:45 & $13: 49$ & 5703193 & Normal & OK & Normal $\left(23^{\circ} \mathrm{C}\right)$ & Normal (54\%) & OK & OK & 140 & -87.971667 & 41.732222 \\
\hline $8 / 28 / 0913: 50$ & $14: 00$ & 5703193 & Normal & OK & Normal $\left(23^{\circ} \mathrm{C}\right)$ & Normal (54\%) & OK & OK & 140 & -87.971667 & 41.732222 \\
\hline $8 / 28 / 09$ 13:55 & $14: 00$ & 5703193 & Normal & OK & Normal $\left(23^{\circ} \mathrm{C}\right)$ & Normal (54\%) & OK & OK & 140 & -87.971667 & 41.732222 \\
\hline $8 / 28 / 09$ 14:00 & $14: 06$ & 5703193 & Normal & OK & Normal $\left(23^{\circ} \mathrm{C}\right)$ & Normal (54\%) & OK & OK & 140 & -88.068611 & 41.684167 \\
\hline 8/28/09 14:05 & $14: 11$ & 5703193 & Normal & OK & Normal $\left(23^{\circ} \mathrm{C}\right)$ & Normal (54\%) & OK & OK & 140 & -88.154167 & 41.640556 \\
\hline $8 / 28 / 09$ 14:10 & $14: 17$ & 5703193 & Normal & OK & Normal $\left(23^{\circ} \mathrm{C}\right)$ & Normal (54\%) & OK & OK & 140 & -88.181111 & 41.568611 \\
\hline $8 / 28 / 09$ 14:15 & $14: 22$ & 5703193 & Normal & OK & Normal $\left(23^{\circ} \mathrm{C}\right)$ & Normal (54\%) & OK & OK & 140 & -88.197778 & 41.493611 \\
\hline $8 / 28 / 0914: 20$ & $14: 22$ & 5703193 & Normal & OK & Normal $\left(23^{\circ} \mathrm{C}\right)$ & Normal (54\%) & OK & OK & 140 & -88.280000 & 41.462500 \\
\hline $8 / 28 / 0914: 25$ & $14: 28$ & 5703193 & Normal & OK & Normal $\left(23^{\circ} \mathrm{C}\right)$ & Normal (54\%) & OK & OK & 140 & -88.371667 & 41.417500 \\
\hline
\end{tabular}




\begin{tabular}{|c|c|c|c|c|c|c|c|c|c|c|c|}
\hline Event Time & at QC Hub & Tag ID & Tag Status & Seal & Temperature & Humidity & Shock & Battery & RSSI & Longitude & Latitude \\
\hline $8 / 28 / 09$ 14:32 & $14: 39$ & 5703193 & Normal & OK & Normal $\left(23^{\circ} \mathrm{C}\right)$ & Normal (54\%) & OK & OK & 140 & -88.422778 & 41.392500 \\
\hline $8 / 28 / 09$ 14:35 & $14: 39$ & 5703193 & Normal & OK & Normal $\left(23^{\circ} \mathrm{C}\right)$ & Normal (54\%) & OK & OK & 140 & -88.422778 & 41.392500 \\
\hline 8/28/09 14:40 & $14: 44$ & 5703193 & Normal & oK & Normal $\left(23^{\circ} \mathrm{C}\right)$ & Normal (54\%) & OK & OK & 140 & -88.422778 & 41.392500 \\
\hline 8/28/09 14:45 & $14: 50$ & 5703193 & Normal & OK & Normal $\left(23^{\circ} \mathrm{C}\right)$ & Normal (54\%) & $\mathrm{OK}$ & $\mathrm{OK}$ & 140 & -88.461389 & 41.378056 \\
\hline 8/28/09 14:50 & $14: 56$ & 5703193 & Normal & $\mathrm{OK}$ & Normal $\left(23^{\circ} \mathrm{C}\right)$ & Normal (54\%) & OK & OK & 140 & -88.575000 & 41.376944 \\
\hline 8/28/09 14:55 & 15:01 & 5703193 & Normal & OK & Normal $\left(25^{\circ} \mathrm{C}\right)$ & Normal $(52 \%)$ & $\mathrm{OK}$ & OK & 140 & -88.717500 & 41.375833 \\
\hline 8/28/09 15:00 & $15: 07$ & 5703193 & Normal & OK & Normal $\left(25^{\circ} \mathrm{C}\right)$ & Normal $(52 \%)$ & OK & OK & 140 & -88.792500 & 41.376944 \\
\hline 8/28/09 15:05 & $15: 12$ & 5703193 & Normal & $\mathrm{OK}$ & Normal $\left(25^{\circ} \mathrm{C}\right)$ & Normal (52\%) & OK & OK & 140 & -88.901944 & 41.368611 \\
\hline 8/28/09 15:10 & $15: 12$ & 5703193 & Normal & $\mathrm{OK}$ & Normal $\left(25^{\circ} \mathrm{C}\right)$ & Normal $(52 \%)$ & OK & OK & 140 & -89.014444 & 41.367500 \\
\hline 8/28/09 15:15 & $15: 18$ & 5703193 & Normal & OK & Normal $\left(25^{\circ} \mathrm{C}\right)$ & Normal $(52 \%)$ & OK & OK & 140 & -89.058333 & 41.413333 \\
\hline $8 / 28 / 09$ 15:20 & $15: 23$ & 5703193 & Normal & OK & Normal $\left(25^{\circ} \mathrm{C}\right)$ & Normal (52\%) & OK & OK & 140 & -89.050833 & 41.460278 \\
\hline $8 / 28 / 09$ 15:25 & $15: 29$ & 5703193 & Normal & OK & Normal $\left(25^{\circ} \mathrm{C}\right)$ & Normal $(52 \%)$ & OK & OK & 140 & -89.058333 & 41.375833 \\
\hline $8 / 28 / 09$ 15:30 & $15: 35$ & 5703193 & Normal & OK & Normal $\left(25^{\circ} \mathrm{C}\right)$ & Normal (52\%) & OK & OK & 140 & -89.125833 & 41.368611 \\
\hline $8 / 28 / 09$ 15:35 & $15: 40$ & 5703193 & Normal & OK & Normal $\left(25^{\circ} \mathrm{C}\right)$ & Normal $(52 \%)$ & OK & OK & 140 & -89.125833 & 41.368611 \\
\hline $8 / 28 / 09$ 15:40 & $15: 46$ & 5703193 & Normal & OK & Normal $\left(25^{\circ} \mathrm{C}\right)$ & Normal (52\%) & OK & OK & 140 & -89.125833 & 41.368611 \\
\hline 8/28/09 15:45 & $15: 51$ & 5703193 & Normal & OK & Normal $\left(25^{\circ} \mathrm{C}\right)$ & Normal $(52 \%)$ & OK & OK & 140 & -89.200000 & 41.364444 \\
\hline $8 / 28 / 09$ 15:50 & $15: 57$ & 5703193 & Normal & OK & Normal $\left(25^{\circ} \mathrm{C}\right)$ & Normal (52\%) & OK & OK & 140 & -89.240556 & 41.371667 \\
\hline 8/28/09 16:00 & $16: 03$ & 5703193 & Normal & OK & Normal $\left(25^{\circ} \mathrm{C}\right)$ & Normal $(52 \%)$ & OK & OK & 140 & -89.458333 & 41.400833 \\
\hline $8 / 28 / 09$ 16:05 & $16: 13$ & 5703193 & Normal & OK & Normal $\left(25^{\circ} \mathrm{C}\right)$ & Normal $(52 \%)$ & OK & OK & 140 & -89.558333 & 41.393611 \\
\hline $8 / 28 / 09$ 16:11 & $16: 14$ & 5703193 & Normal & OK & Normal $\left(25^{\circ} \mathrm{C}\right)$ & Normal $(52 \%)$ & OK & OK & 140 & -89.558333 & 41.393611 \\
\hline $8 / 28 / 09$ 16:15 & $16: 19$ & 5703193 & Normal & OK & Normal $\left(25^{\circ} \mathrm{C}\right)$ & Normal (52\%) & OK & OK & 140 & -89.558333 & 41.393611 \\
\hline $8 / 28 / 09$ 16:20 & $16: 25$ & 5703193 & Normal & OK & Normal $\left(25^{\circ} \mathrm{C}\right)$ & Normal (52\%) & OK & OK & 140 & -89.558333 & 41.393611 \\
\hline $8 / 28 / 09$ 16:25 & $16: 30$ & 5703193 & Normal & OK & Normal $\left(25^{\circ} \mathrm{C}\right)$ & Normal (52\%) & OK & OK & 140 & -89.623889 & 41.394722 \\
\hline $8 / 28 / 09$ 16:30 & $16: 36$ & 5703193 & Normal & OK & Normal $\left(25^{\circ} \mathrm{C}\right)$ & Normal (52\%) & OK & OK & 140 & -89.725833 & 41.387500 \\
\hline $8 / 28 / 09$ 16:35 & $16: 41$ & 5703193 & Normal & OK & Normal $\left(25^{\circ} \mathrm{C}\right)$ & Normal $(52 \%)$ & OK & OK & 140 & -89.834167 & 41.388333 \\
\hline $8 / 28 / 09$ 16:40 & $16: 47$ & 5703193 & Normal & OK & Normal $\left(25^{\circ} \mathrm{C}\right)$ & Normal $(52 \%)$ & OK & OK & 140 & -89.941667 & 41.416667 \\
\hline $8 / 28 / 09$ 16:45 & $16: 52$ & 5703193 & Normal & OK & Normal $\left(25^{\circ} \mathrm{C}\right)$ & Normal $(52 \%)$ & OK & OK & 140 & -90.050000 & 41.413333 \\
\hline $8 / 28 / 0916: 50$ & $16: 52$ & 5703193 & Normal & OK & Normal $\left(25^{\circ} \mathrm{C}\right)$ & Normal $(52 \%)$ & OK & OK & 140 & -90.157222 & 41.430000 \\
\hline $8 / 28 / 09$ 16:55 & $16: 58$ & 5703193 & Normal & OK & Normal $\left(25^{\circ} \mathrm{C}\right)$ & Normal $(52 \%)$ & OK & OK & 140 & -90.267500 & 41.440556 \\
\hline $8 / 28 / 09$ 17:00 & $17: 09$ & 5703193 & Normal & OK & Normal $\left(25^{\circ} \mathrm{C}\right)$ & Normal $(52 \%)$ & OK & OK & 140 & -90.338333 & 41.476944 \\
\hline 8/28/09 17:05 & $17: 09$ & 5703193 & Normal & OK & Normal $\left(25^{\circ} \mathrm{C}\right)$ & Normal (52\%) & OK & OK & 140 & -90.334167 & 41.486389 \\
\hline $8 / 28 / 09$ 17:11 & $17: 15$ & 5703193 & Normal & OK & Normal $\left(25^{\circ} \mathrm{C}\right)$ & Normal (52\%) & OK & OK & 140 & -90.334167 & 41.486389 \\
\hline $8 / 28 / 09$ 17:15 & $17: 20$ & 5703193 & Normal & OK & Normal $\left(25^{\circ} \mathrm{C}\right)$ & Normal (52\%) & OK & OK & 140 & -90.334167 & 41.486389 \\
\hline $8 / 28 / 09$ 17:20 & $17: 26$ & 5703193 & Normal & OK & Normal $\left(25^{\circ} \mathrm{C}\right)$ & Normal (52\%) & OK & OK & 140 & -90.334167 & 41.486389 \\
\hline $8 / 28 / 09$ 17:25 & $17: 31$ & 5703193 & Normal & OK & Normal $\left(25^{\circ} \mathrm{C}\right)$ & Normal (52\%) & OK & OK & 140 & -90.340556 & 41.511389 \\
\hline $8 / 28 / 09$ 17:30 & $17: 37$ & 5703193 & Normal & OK & Normal $\left(25^{\circ} \mathrm{C}\right)$ & Normal $(52 \%)$ & OK & OK & 140 & -90.234167 & 41.562500 \\
\hline 8/28/09 17:35 & $17: 42$ & 5703193 & Normal & OK & Normal $\left(25^{\circ} \mathrm{C}\right)$ & Normal (52\%) & OK & OK & 140 & -90.209167 & 41.581111 \\
\hline $8 / 28 / 09$ 17:40 & $17: 42$ & 5703193 & Normal & OK & Normal $\left(25^{\circ} \mathrm{C}\right)$ & Normal $(52 \%)$ & OK & OK & 140 & -90.163333 & 41.642500 \\
\hline $8 / 28 / 09$ 17:45 & $17: 48$ & 5703193 & Normal & OK & Normal $\left(25^{\circ} \mathrm{C}\right)$ & Normal $(52 \%)$ & OK & OK & 140 & -90.080000 & 41.690556 \\
\hline 8/28/09 17:50 & $17: 53$ & 5703193 & Normal & OK & Normal $\left(25^{\circ} \mathrm{C}\right)$ & Normal (52\%) & OK & OK & 140 & -89.983333 & 41.718611 \\
\hline 8/28/09 17:55 & $17: 59$ & 5703193 & Normal & OK & Normal $\left(28^{\circ} \mathrm{C}\right)$ & Normal $(49 \%)$ & OK & OK & 140 & -89.876944 & 41.744722 \\
\hline 8/28/09 18:00 & $18: 04$ & 5703193 & Normal & OK & Normal $\left(28^{\circ} \mathrm{C}\right)$ & Normal (49\%) & OK & OK & 140 & -89.775000 & 41.757222 \\
\hline 8/28/09 18:05 & $18: 10$ & 5703193 & Normal & OK & Normal $\left(28^{\circ} \mathrm{C}\right)$ & Normal (49\%) & OK & OK & 140 & -89.689444 & 41.750833 \\
\hline $8 / 28 / 09$ 18:10 & $18: 15$ & 5703193 & Normal & OK & Normal $\left(28^{\circ} \mathrm{C}\right)$ & Normal (49\%) & OK & OK & 140 & -89.689444 & 41.762500 \\
\hline 8/28/09 18:15 & $18: 21$ & 5703193 & Normal & OK & Normal $\left(28^{\circ} \mathrm{C}\right)$ & Normal (49\%) & OK & OK & 140 & -89.678056 & 41.750833 \\
\hline $8 / 28 / 09$ 18:20 & $18: 26$ & 5703193 & Normal & OK & Normal $\left(28^{\circ} \mathrm{C}\right)$ & Normal (49\%) & OK & OK & 140 & -89.635278 & 41.759167 \\
\hline $8 / 28 / 09$ 18:25 & $18: 32$ & 5703193 & Normal & OK & Normal $\left(28^{\circ} \mathrm{C}\right)$ & Normal (49\%) & OK & OK & 140 & -89.532222 & 41.789444 \\
\hline 8/28/09 18:30 & $18: 38$ & 5703193 & Normal & OK & Normal $\left(28^{\circ} \mathrm{C}\right)$ & Normal $(49 \%)$ & OK & OK & 140 & -89.432222 & 41.822778 \\
\hline $8 / 28 / 09$ 18:35 & $18: 38$ & 5703193 & Normal & OK & Normal $\left(28^{\circ} \mathrm{C}\right)$ & Normal $(49 \%)$ & OK & OK & 140 & -89.336389 & 41.822778 \\
\hline 8/28/09 18:40 & $18: 43$ & 5703193 & Normal & OK & Normal $\left(28^{\circ} \mathrm{C}\right)$ & Normal $(49 \%)$ & OK & OK & 140 & -89.231111 & 41.850833 \\
\hline 8/28/09 18:45 & $18: 48$ & 5703193 & Normal & OK & Normal $\left(29^{\circ} \mathrm{C}\right)$ & Normal (47\%) & OK & OK & 140 & -89.125000 & 41.883333 \\
\hline 8/28/09 18:50 & $18: 54$ & 5703193 & Normal & OK & Normal $\left(29^{\circ} \mathrm{C}\right)$ & Normal (47\%) & OK & OK & 140 & -89.025000 & 41.904167 \\
\hline $8 / 28 / 09$ 18:55 & $19: 00$ & 5703193 & Normal & OK & Normal $\left(29^{\circ} \mathrm{C}\right)$ & Normal $(47 \%)$ & OK & OK & 140 & -88.913333 & 41.900000 \\
\hline $8 / 28 / 09$ 19:00 & 19:05 & 5703193 & Normal & OK & Normal $\left(29^{\circ} \mathrm{C}\right)$ & Normal (47\%) & OK & OK & 140 & -88.814444 & 41.900000 \\
\hline $8 / 28 / 09$ 19:05 & $19: 11$ & 5703193 & Normal & OK & Normal $\left(29^{\circ} \mathrm{C}\right)$ & Normal $(47 \%)$ & OK & OK & 140 & -88.739444 & 41.900000 \\
\hline 8/28/09 19:10 & $19: 16$ & 5703193 & Normal & OK & Normal $\left(29^{\circ} \mathrm{C}\right)$ & Normal (47\%) & OK & OK & 140 & -88.739444 & 41.900000 \\
\hline 8/28/09 19:15 & $19: 22$ & 5703193 & Normal & OK & Normal $\left(28^{\circ} \mathrm{C}\right)$ & Normal (46\%) & OK & OK & 140 & -88.737500 & 41.900000 \\
\hline $8 / 28 / 09$ 19:20 & $19: 27$ & 5703193 & Normal & OK & Normal $\left(28^{\circ} \mathrm{C}\right)$ & Normal (46\%) & OK & OK & 140 & -88.737500 & 41.900000 \\
\hline $8 / 28 / 09$ 19:25 & $19: 33$ & 5703193 & Normal & oK & Normal $\left(28^{\circ} \mathrm{C}\right)$ & Normal (46\%) & OK & OK & 140 & -88.639444 & 41.894722 \\
\hline $8 / 28 / 09$ 19:30 & $19: 38$ & 5703193 & Normal & OK & Normal $\left(28^{\circ} \mathrm{C}\right)$ & Normal $(46 \%)$ & OK & OK & 140 & -88.538333 & 41.859167 \\
\hline 8/28/09 19:35 & $19: 38$ & 5703193 & Normal & OK & Normal $\left(28^{\circ} \mathrm{C}\right)$ & Normal $(46 \%)$ & OK & OK & 140 & -88.448889 & 41.812500 \\
\hline $8 / 28 / 09$ 19:40 & $19: 44$ & 5703193 & Normal & OK & Normal $\left(28^{\circ} \mathrm{C}\right)$ & Normal $(46 \%)$ & OK & OK & 140 & -88.347778 & 41.794722 \\
\hline $8 / 28 / 09$ 19:45 & $19: 49$ & 5703193 & Normal & OK & Normal $\left(28^{\circ} \mathrm{C}\right)$ & Normal $(46 \%)$ & OK & OK & 140 & -88.261389 & 41.800000 \\
\hline $8 / 28 / 09$ 19:50 & $19: 55$ & 5703193 & Normal & OK & Normal $\left(28^{\circ} \mathrm{C}\right)$ & Normal $(46 \%)$ & OK & OK & 140 & -88.156111 & 41.806111 \\
\hline $8 / 28 / 09$ 19:55 & $20: 00$ & 5703193 & Normal & OK & Normal $\left(28^{\circ} \mathrm{C}\right)$ & Normal $(46 \%)$ & OK & OK & 140 & -88.062500 & 41.810278 \\
\hline $8 / 28 / 0920: 00$ & $20: 06$ & 5703193 & Normal & OK & Normal $\left(28^{\circ} \mathrm{C}\right)$ & Normal (46\%) & OK & OK & 140 & -88.054167 & 41.807222 \\
\hline 8/28/09 20:05 & $20: 11$ & 5703193 & Normal & OK & Normal $\left(29^{\circ} \mathrm{C}\right)$ & Normal (47\%) & OK & OK & 140 & -88.054167 & 41.781111 \\
\hline 8/28/09 20:10 & $20: 22$ & 5703193 & Normal & OK & Normal $\left(29^{\circ} \mathrm{C}\right)$ & Normal $(47 \%)$ & OK & OK & 140 & -88.035278 & 41.732222 \\
\hline $8 / 28 / 0913: 40$ & $13: 44$ & 5703194 & Normal & OK & Normal $\left(23^{\circ} \mathrm{C}\right)$ & Normal $(52 \%)$ & $\overline{\mathrm{OK}}$ & OK & 141 & -87.971667 & 41.732222 \\
\hline $8 / 28 / 09$ 13:45 & $13: 49$ & 5703194 & Normal & OK & Normal $\left(23^{\circ} \mathrm{C}\right)$ & Normal $(52 \%)$ & OK & OK & 141 & -87.971667 & 41.732222 \\
\hline $8 / 28 / 09$ 13:50 & $14: 00$ & 5703194 & Normal & OK & Normal $\left(23^{\circ} \mathrm{C}\right)$ & Normal (52\%) & OK & OK & 141 & -87.971667 & 41.732222 \\
\hline $8 / 28 / 09$ 13:55 & $14: 00$ & 5703194 & Normal & OK & Normal $\left(23^{\circ} \mathrm{C}\right)$ & Normal $(52 \%)$ & OK & OK & 141 & -87.971667 & 41.732222 \\
\hline $8 / 28 / 0914: 00$ & $14: 06$ & 5703194 & Normal & OK & Normal $\left(23^{\circ} \mathrm{C}\right)$ & Normal $(52 \%)$ & OK & OK & 141 & -88.068611 & 41.684167 \\
\hline 8/28/09 14:05 & $14: 11$ & 5703194 & Normal & OK & Normal $\left(23^{\circ} \mathrm{C}\right)$ & Normal (52\%) & OK & OK & 141 & -88.154167 & 41.640556 \\
\hline $8 / 28 / 09$ 14:10 & $14: 17$ & 5703194 & Normal & OK & Normal $\left(23^{\circ} \mathrm{C}\right)$ & Normal $(52 \%)$ & OK & OK & 141 & -88.181111 & 41.568611 \\
\hline $8 / 28 / 09$ 14:15 & $14: 22$ & 5703194 & Normal & OK & Normal $\left(23^{\circ} \mathrm{C}\right)$ & Normal $(52 \%)$ & OK & OK & 141 & -88.197778 & 41.493611 \\
\hline $8 / 28 / 0914: 20$ & $14: 22$ & 5703194 & Normal & OK & Normal $\left(23^{\circ} \mathrm{C}\right)$ & Normal $(52 \%)$ & OK & OK & 141 & -88.280000 & 41.462500 \\
\hline $8 / 28 / 09$ 14:25 & $14: 28$ & 5703194 & Normal & OK & Normal $\left(23^{\circ} \mathrm{C}\right)$ & Normal $(52 \%)$ & OK & OK & 141 & -88.371667 & 41.417500 \\
\hline $8 / 28 / 0914: 30$ & $14: 34$ & 5703194 & Alarm & OK & Normal $\left(23^{\circ} \mathrm{C}\right)$ & Normal $(52 \%)$ & Shocked & Low & 141 & -88.422778 & 41.392500 \\
\hline $8 / 28 / 09$ 14:32 & $14: 39$ & 5703194 & Normal & OK & Normal $\left(23^{\circ} \mathrm{C}\right)$ & Normal $(50 \%)$ & OK & $\mathrm{OK}$ & 141 & -88.422778 & 41.392500 \\
\hline 8/28/09 14:35 & $14: 39$ & 5703194 & Normal & oK & Normal $\left(23^{\circ} \mathrm{C}\right)$ & Normal $(50 \%)$ & OK & OK & 141 & -88.422778 & 41.392500 \\
\hline
\end{tabular}




\begin{tabular}{|c|c|c|c|c|c|c|c|c|c|c|c|}
\hline Event Time & at QC Hub & Tag ID & Tag Status & Seal & Temperature & Humidity & Shock & Battery & RSSI & Longitude & Latitude \\
\hline $8 / 28 / 0914: 40$ & $14: 44$ & 5703194 & Normal & OK & Normal $\left(24^{\circ} \mathrm{C}\right)$ & Normal (51\%) & OK & OK & 141 & -88.422778 & 41.392500 \\
\hline 8/28/09 14:45 & $14: 50$ & 5703194 & Normal & OK & Normal $\left(24^{\circ} \mathrm{C}\right)$ & Normal (51\%) & OK & OK & 141 & -88.461389 & 41.378056 \\
\hline $8 / 28 / 0914: 50$ & $14: 56$ & 5703194 & Normal & OK & Normal $\left(24^{\circ} \mathrm{C}\right)$ & Normal (51\%) & OK & OK & 141 & -88.575000 & 41.376944 \\
\hline $8 / 28 / 0914: 55$ & $15: 01$ & 5703194 & Normal & OK & Normal $\left(24^{\circ} \mathrm{C}\right)$ & Normal (50\%) & OK & OK & 141 & -88.717500 & 41.375833 \\
\hline $8 / 28 / 09$ 15:00 & $15: 07$ & 5703194 & Normal & $\mathrm{OK}$ & Normal $\left(24^{\circ} \mathrm{C}\right)$ & Normal $(50 \%)$ & $\mathrm{OK}$ & OK & 141 & -88.792500 & 41.376944 \\
\hline 8/28/09 15:05 & $15: 12$ & 5703194 & Normal & OK & Normal $\left(24^{\circ} \mathrm{C}\right)$ & Normal (50\%) & OK & OK & 141 & -88.901944 & 41.368611 \\
\hline 8/28/09 15:10 & $15: 12$ & 5703194 & Normal & OK & Normal $\left(24^{\circ} \mathrm{C}\right)$ & Normal $(50 \%)$ & OK & OK & 141 & -89.014444 & 41.367500 \\
\hline 8/28/09 15:15 & $15: 18$ & 5703194 & Normal & OK & Normal $\left(24^{\circ} \mathrm{C}\right)$ & Normal $(50 \%)$ & OK & OK & 141 & -89.058333 & 41.413333 \\
\hline $8 / 28 / 09$ 15:20 & $15: 23$ & 5703194 & Normal & $\mathrm{OK}$ & Normal $\left(24^{\circ} \mathrm{C}\right)$ & Normal $(50 \%)$ & OK & OK & 141 & -89.050833 & 41.460278 \\
\hline $8 / 28 / 09$ 15:25 & $15: 29$ & 5703194 & Normal & $\mathrm{OK}$ & Normal $\left(24^{\circ} \mathrm{C}\right)$ & Normal $(50 \%)$ & OK & OK & 141 & -89.058333 & 41.375833 \\
\hline $8 / 28 / 09$ 15:30 & $15: 35$ & 5703194 & Normal & OK & Normal $\left(24^{\circ} \mathrm{C}\right)$ & Normal $(50 \%)$ & OK & OK & 141 & -89.125833 & 41.368611 \\
\hline 8/28/09 15:35 & $15: 40$ & 5703194 & Normal & $\mathrm{OK}$ & Normal $\left(24^{\circ} \mathrm{C}\right)$ & Normal $(50 \%)$ & OK & OK & 141 & -89.125833 & 41.368611 \\
\hline 8/28/09 15:40 & $15: 46$ & 5703194 & Normal & $\mathrm{OK}$ & Normal $\left(24^{\circ} \mathrm{C}\right)$ & Normal $(50 \%)$ & OK & OK & 141 & -89.125833 & 41.368611 \\
\hline $8 / 28 / 09$ 15:45 & $15: 51$ & 5703194 & Normal & OK & Normal $\left(24^{\circ} \mathrm{C}\right)$ & Normal (50\%) & OK & OK & 141 & -89.200000 & 41.364444 \\
\hline $8 / 28 / 09$ 15:50 & $15: 57$ & 5703194 & Normal & OK & Normal $\left(24^{\circ} \mathrm{C}\right)$ & Normal (50\%) & OK & OK & 141 & -89.240556 & 41.371667 \\
\hline $8 / 28 / 09$ 16:00 & $16: 03$ & 5703194 & Normal & OK & Normal $\left(24^{\circ} \mathrm{C}\right)$ & Normal $(50 \%)$ & $\mathrm{OK}$ & OK & 141 & -89.458333 & 41.400833 \\
\hline $8 / 28 / 09$ 16:05 & $16: 13$ & 5703194 & Normal & OK & Normal $\left(24^{\circ} \mathrm{C}\right)$ & Normal $(50 \%)$ & $\mathrm{OK}$ & OK & 141 & -89.558333 & 41.393611 \\
\hline 8/28/09 16:11 & $16: 14$ & 5703194 & Normal & OK & Normal $\left(24^{\circ} \mathrm{C}\right)$ & Normal $(50 \%)$ & OK & OK & 141 & -89.558333 & 41.393611 \\
\hline 8/28/09 16:15 & $16: 19$ & 5703194 & Normal & OK & Normal $\left(24^{\circ} \mathrm{C}\right)$ & Normal $(50 \%)$ & OK & OK & 141 & -89.558333 & 41.393611 \\
\hline $8 / 28 / 09$ 16:20 & $16: 25$ & 5703194 & Normal & OK & Normal $\left(24^{\circ} \mathrm{C}\right)$ & Normal $(50 \%)$ & OK & OK & 141 & -89.558333 & 41.393611 \\
\hline $8 / 28 / 09$ 16:25 & $16: 30$ & 5703194 & Normal & $\mathrm{OK}$ & Normal $\left(24^{\circ} \mathrm{C}\right)$ & Normal $(50 \%)$ & OK & OK & 141 & -89.623889 & 41.394722 \\
\hline $8 / 28 / 09$ 16:30 & $16: 36$ & 5703194 & Normal & OK & Normal $\left(24^{\circ} \mathrm{C}\right)$ & Normal (50\%) & OK & OK & 141 & -89.725833 & 41.387500 \\
\hline $8 / 28 / 09$ 16:35 & $16: 41$ & 5703194 & Normal & OK & Normal $\left(24^{\circ} \mathrm{C}\right)$ & Normal $(50 \%)$ & OK & OK & 141 & -89.834167 & 41.388333 \\
\hline $8 / 28 / 0916: 40$ & $16: 47$ & 5703194 & Normal & OK & Normal $\left(24^{\circ} \mathrm{C}\right)$ & Normal (50\%) & OK & OK & 141 & -89.941667 & 41.416667 \\
\hline $8 / 28 / 09$ 16:45 & $16: 52$ & 5703194 & Normal & OK & Normal $\left(24^{\circ} \mathrm{C}\right)$ & Normal $(50 \%)$ & OK & OK & 141 & -90.050000 & 41.413333 \\
\hline $8 / 28 / 09$ 16:50 & $16: 52$ & 5703194 & Normal & OK & Normal $\left(24^{\circ} \mathrm{C}\right)$ & Normal (50\%) & OK & OK & 141 & -90.157222 & 41.430000 \\
\hline $8 / 28 / 0916: 55$ & $16: 58$ & 5703194 & Normal & OK & Normal $\left(24^{\circ} \mathrm{C}\right)$ & Normal (50\%) & OK & OK & 141 & -90.267500 & 41.440556 \\
\hline $8 / 28 / 09$ 17:00 & 17:09 & 5703194 & Normal & OK & Normal $\left(24^{\circ} \mathrm{C}\right)$ & Normal $(50 \%)$ & OK & OK & 141 & -90.338333 & 41.476944 \\
\hline $8 / 28 / 09$ 17:05 & 17:09 & 5703194 & Normal & OK & Normal $\left(24^{\circ} \mathrm{C}\right)$ & Normal (50\%) & OK & OK & 141 & -90.334167 & 41.486389 \\
\hline $8 / 28 / 09$ 17:11 & $17: 15$ & 5703194 & Normal & OK & Normal $\left(24^{\circ} \mathrm{C}\right)$ & Normal (50\%) & OK & OK & 141 & -90.334167 & 41.486389 \\
\hline $8 / 28 / 09$ 17:15 & $17: 20$ & 5703194 & Normal & OK & Normal $\left(24^{\circ} \mathrm{C}\right)$ & Normal $(50 \%)$ & OK & OK & 141 & -90.334167 & 41.486389 \\
\hline $8 / 28 / 09$ 17:20 & $17: 26$ & 5703194 & Normal & OK & Normal $\left(24^{\circ} \mathrm{C}\right)$ & Normal $(50 \%)$ & OK & OK & 141 & -90.334167 & 41.486389 \\
\hline $8 / 28 / 09$ 17:25 & $17: 31$ & 5703194 & Normal & OK & Normal $\left(24^{\circ} \mathrm{C}\right)$ & Normal $(50 \%)$ & OK & OK & 141 & -90.340556 & 41.511389 \\
\hline $8 / 28 / 09$ 17:30 & $17: 37$ & 5703194 & Normal & OK & Normal $\left(24^{\circ} \mathrm{C}\right)$ & Normal $(50 \%)$ & OK & OK & 141 & -90.234167 & 41.562500 \\
\hline $8 / 28 / 09$ 17:35 & $17: 42$ & 5703194 & Normal & OK & $\operatorname{Normal}\left(24^{\circ} \mathrm{C}\right)$ & Normal (50\%) & OK & OK & 141 & -90.209167 & 41.581111 \\
\hline 8/28/09 17:40 & $17: 42$ & 5703194 & Normal & OK & Normal $\left(24^{\circ} \mathrm{C}\right)$ & Normal $(50 \%)$ & OK & OK & 141 & -90.163333 & 41.642500 \\
\hline $8 / 28 / 09$ 17:45 & $17: 48$ & 5703194 & Normal & OK & Normal $\left(24^{\circ} \mathrm{C}\right)$ & Normal (50\%) & OK & OK & 141 & -90.080000 & 41.690556 \\
\hline $8 / 28 / 09$ 17:50 & $17: 53$ & 5703194 & Normal & OK & Normal $\left(24^{\circ} \mathrm{C}\right)$ & Normal (50\%) & OK & OK & 141 & -89.983333 & 41.718611 \\
\hline $8 / 28 / 09$ 17:55 & $17: 59$ & 5703194 & Normal & OK & Normal $\left(27^{\circ} \mathrm{C}\right)$ & Normal $(50 \%)$ & OK & OK & 141 & -89.876944 & 41.744722 \\
\hline $8 / 28 / 0918: 00$ & $18: 04$ & 5703194 & Normal & OK & Normal $\left(27^{\circ} \mathrm{C}\right)$ & Normal $(50 \%)$ & OK & OK & 141 & -89.775000 & 41.757222 \\
\hline $8 / 28 / 09$ 18:05 & $18: 10$ & 5703194 & Normal & OK & Normal $\left(27^{\circ} \mathrm{C}\right)$ & Normal $(50 \%)$ & OK & OK & 141 & -89.689444 & 41.750833 \\
\hline $8 / 28 / 09$ 18:10 & $18: 15$ & 5703194 & Normal & OK & Normal $\left(27^{\circ} \mathrm{C}\right)$ & Normal (50\%) & OK & OK & 141 & -89.689444 & 41.762500 \\
\hline 8/28/09 18:15 & $18: 21$ & 5703194 & Normal & OK & Normal $\left(27^{\circ} \mathrm{C}\right)$ & Normal $(50 \%)$ & OK & OK & 141 & -89.678056 & 41.750833 \\
\hline $8 / 28 / 09$ 18:20 & $18: 26$ & 5703194 & Normal & OK & Normal $\left(27^{\circ} \mathrm{C}\right)$ & Normal $(50 \%)$ & OK & OK & 141 & -89.635278 & 41.759167 \\
\hline $8 / 28 / 09$ 18:25 & $18: 32$ & 5703194 & Normal & OK & Normal $\left(27^{\circ} \mathrm{C}\right)$ & Normal (50\%) & OK & OK & 141 & -89.532222 & 41.789444 \\
\hline $8 / 28 / 09$ 18:30 & $18: 38$ & 5703194 & Normal & OK & Normal $\left(27^{\circ} \mathrm{C}\right)$ & Normal (50\%) & OK & OK & 141 & -89.432222 & 41.822778 \\
\hline $8 / 28 / 09$ 18:35 & $18: 38$ & 5703194 & Normal & OK & Normal $\left(27^{\circ} \mathrm{C}\right)$ & Normal (50\%) & OK & OK & 141 & -89.336389 & 41.822778 \\
\hline $8 / 28 / 09$ 18:40 & $18: 43$ & 5703194 & Normal & OK & Normal $\left(27^{\circ} \mathrm{C}\right)$ & Normal (50\%) & OK & OK & 141 & -89.231111 & 41.850833 \\
\hline $8 / 28 / 0918: 45$ & $18: 48$ & 5703194 & Normal & OK & Normal $\left(27^{\circ} \mathrm{C}\right)$ & Normal (49\%) & OK & OK & 141 & -89.125000 & 41.883333 \\
\hline $8 / 28 / 09$ 18:50 & $18: 54$ & 5703194 & Normal & OK & Normal $\left(27^{\circ} \mathrm{C}\right)$ & Normal (49\%) & OK & OK & 141 & -89.025000 & 41.904167 \\
\hline 8/28/09 18:55 & 19:00 & 5703194 & Normal & OK & Normal $\left(27^{\circ} \mathrm{C}\right)$ & Normal $(49 \%)$ & OK & OK & 141 & -88.913333 & 41.900000 \\
\hline $8 / 28 / 09$ 19:00 & 19:05 & 5703194 & Normal & OK & Normal $\left(27^{\circ} \mathrm{C}\right)$ & Normal (49\%) & OK & OK & 141 & -88.814444 & 41.900000 \\
\hline 8/28/09 19:05 & 19:11 & 5703194 & Normal & OK & Normal $\left(27^{\circ} \mathrm{C}\right)$ & Normal $(49 \%)$ & OK & OK & 141 & -88.739444 & 41.900000 \\
\hline $8 / 28 / 09$ 19:10 & $19: 16$ & 5703194 & Normal & OK & Normal $\left(27^{\circ} \mathrm{C}\right)$ & Normal (49\%) & OK & OK & 141 & -88.739444 & 41.900000 \\
\hline $8 / 28 / 09$ 19:15 & $19: 22$ & 5703194 & Normal & OK & Normal $\left(27^{\circ} \mathrm{C}\right)$ & Normal (49\%) & OK & OK & 141 & -88.737500 & 41.900000 \\
\hline $8 / 28 / 09$ 19:20 & 19:27 & 5703194 & Normal & OK & Normal $\left(27^{\circ} \mathrm{C}\right)$ & Normal (49\%) & OK & OK & 141 & -88.737500 & 41.900000 \\
\hline $8 / 28 / 09$ 19:25 & $19: 33$ & 5703194 & Normal & OK & Normal $\left(27^{\circ} \mathrm{C}\right)$ & Normal (49\%) & OK & OK & 141 & -88.639444 & 41.894722 \\
\hline $8 / 28 / 09$ 19:30 & $19: 38$ & 5703194 & Normal & OK & Normal $\left(27^{\circ} \mathrm{C}\right)$ & Normal (49\%) & OK & OK & 141 & -88.538333 & 41.859167 \\
\hline $8 / 28 / 09$ 19:35 & $19: 38$ & 5703194 & Normal & OK & Normal $\left(27^{\circ} \mathrm{C}\right)$ & Normal (49\%) & OK & OK & 141 & -88.448889 & 41.812500 \\
\hline $8 / 28 / 09$ 19:40 & $19: 44$ & 5703194 & Normal & OK & Normal $\left(27^{\circ} \mathrm{C}\right)$ & Normal $(49 \%)$ & OK & OK & 141 & -88.347778 & 41.794722 \\
\hline $8 / 28 / 09$ 19:45 & $19: 49$ & 5703194 & Normal & OK & Normal $\left(27^{\circ} \mathrm{C}\right)$ & Normal $(49 \%)$ & OK & OK & 141 & -88.261389 & 41.800000 \\
\hline $8 / 28 / 09$ 19:50 & 19:55 & 5703194 & Normal & OK & Normal $\left(27^{\circ} \mathrm{C}\right)$ & Normal (49\%) & OK & OK & 141 & -88.156111 & 41.806111 \\
\hline $8 / 28 / 09$ 19:55 & $20: 00$ & 5703194 & Normal & OK & Normal $\left(27^{\circ} \mathrm{C}\right)$ & Normal $(49 \%)$ & OK & OK & 141 & -88.062500 & 41.810278 \\
\hline $8 / 28 / 09$ 20:00 & 20:06 & 5703194 & Normal & OK & Normal $\left(27^{\circ} \mathrm{C}\right)$ & Normal $(49 \%)$ & OK & OK & 141 & -88.054167 & 41.807222 \\
\hline 8/28/09 20:05 & $20: 11$ & 5703194 & Normal & OK & Normal $\left(27^{\circ} \mathrm{C}\right)$ & Normal $(50 \%)$ & OK & OK & 141 & -88.054167 & 41.781111 \\
\hline $8 / 28 / 09$ 20:10 & $20: 22$ & 5703194 & Normal & OK & Normal $\left(27^{\circ} \mathrm{C}\right)$ & Normal $(50 \%)$ & OK & OK & 141 & -88.035278 & 41.732222 \\
\hline $8 / 28 / 0913: 40$ & $13: 44$ & 5703195 & Normal & $\overline{O K}$ & Normal $\left(23^{\circ} \mathrm{C}\right)$ & Normal (51\%) & OK & $\overline{\mathrm{OK}}$ & 139 & -87.971667 & 41.732222 \\
\hline $8 / 28 / 0913: 45$ & $13: 49$ & 5703195 & Normal & OK & Normal $\left(23^{\circ} \mathrm{C}\right)$ & Normal (51\%) & OK & OK & 139 & -87.971667 & 41.732222 \\
\hline $8 / 28 / 09$ 13:50 & $14: 00$ & 5703195 & Normal & OK & Normal $\left(23^{\circ} \mathrm{C}\right)$ & Normal (51\%) & OK & OK & 139 & -87.971667 & 41.732222 \\
\hline $8 / 28 / 09$ 13:55 & $14: 00$ & 5703195 & Normal & OK & Normal $\left(23^{\circ} \mathrm{C}\right)$ & Normal $(51 \%)$ & OK & OK & 139 & -87.971667 & 41.732222 \\
\hline $8 / 28 / 0914: 00$ & $14: 06$ & 5703195 & Normal & OK & Normal $\left(23^{\circ} \mathrm{C}\right)$ & Normal (51\%) & OK & OK & 139 & -88.068611 & 41.684167 \\
\hline $8 / 28 / 09$ 14:05 & $14: 11$ & 5703195 & Normal & OK & Normal $\left(23^{\circ} \mathrm{C}\right)$ & Normal (51\%) & OK & OK & 139 & -88.154167 & 41.640556 \\
\hline $8 / 28 / 0914: 10$ & $14: 17$ & 5703195 & Normal & OK & Normal $\left(23^{\circ} \mathrm{C}\right)$ & Normal (51\%) & OK & OK & 139 & -88.181111 & 41.568611 \\
\hline $8 / 28 / 09$ 14:15 & $14: 22$ & 5703195 & Normal & OK & Normal $\left(23^{\circ} \mathrm{C}\right)$ & Normal (51\%) & OK & OK & 139 & -88.197778 & 41.493611 \\
\hline $8 / 28 / 0914: 20$ & $14: 22$ & 5703195 & Normal & OK & Normal $\left(23^{\circ} \mathrm{C}\right)$ & Normal (51\%) & OK & OK & 139 & -88.280000 & 41.462500 \\
\hline $8 / 28 / 09$ 14:25 & $14: 28$ & 5703195 & Normal & OK & Normal $\left(23^{\circ} \mathrm{C}\right)$ & Normal (51\%) & OK & OK & 139 & -88.371667 & 41.417500 \\
\hline $8 / 28 / 09$ 14:32 & $14: 39$ & 5703195 & Normal & OK & Normal $\left(23^{\circ} \mathrm{C}\right)$ & Normal (51\%) & OK & OK & 139 & -88.422778 & 41.392500 \\
\hline $8 / 28 / 09$ 14:35 & $14: 39$ & 5703195 & Normal & OK & Normal $\left(23^{\circ} \mathrm{C}\right)$ & Normal (51\%) & OK & OK & 139 & -88.422778 & 41.392500 \\
\hline $8 / 28 / 0914: 40$ & $14: 44$ & 5703195 & Normal & OK & Normal $\left(23^{\circ} \mathrm{C}\right)$ & Normal (51\%) & OK & OK & 139 & -88.422778 & 41.392500 \\
\hline $8 / 28 / 0914: 45$ & $14: 50$ & 5703195 & Normal & OK & Normal $\left(23^{\circ} \mathrm{C}\right)$ & Normal (51\%) & OK & OK & 139 & -88.461389 & 41.378056 \\
\hline $8 / 28 / 09$ 14:50 & $14: 56$ & 5703195 & Normal & OK & Normal $\left(23^{\circ} \mathrm{C}\right)$ & Normal (51\%) & OK & OK & 139 & -88.575000 & 41.376944 \\
\hline
\end{tabular}




\begin{tabular}{|c|c|c|c|c|c|c|c|c|c|c|c|}
\hline Event Time & at QC Hub & Tag ID & Tag Status & Seal & Temperature & Humidity & Shock & Battery & RSSI & Longitude & Latitude \\
\hline $8 / 28 / 0914: 55$ & $15: 01$ & 5703195 & Normal & $\mathrm{OK}$ & Normal $\left(23^{\circ} \mathrm{C}\right)$ & Normal (47\%) & OK & OK & 139 & -88.717500 & 41.375833 \\
\hline $8 / 28 / 09$ 15:00 & $15: 07$ & 5703195 & Normal & OK & Normal $\left(23^{\circ} \mathrm{C}\right)$ & Normal $(47 \%)$ & OK & OK & 139 & -88.792500 & 41.376944 \\
\hline 8/28/09 15:05 & $15: 12$ & 5703195 & Normal & OK & Normal $\left(23^{\circ} \mathrm{C}\right)$ & Normal $(47 \%)$ & OK & OK & 139 & -88.901944 & 41.368611 \\
\hline 8/28/09 15:10 & $15: 12$ & 5703195 & Normal & OK & Normal $\left(23^{\circ} \mathrm{C}\right)$ & Normal (47\%) & OK & OK & 139 & -89.014444 & 41.367500 \\
\hline 8/28/09 15:15 & $15: 18$ & 5703195 & Normal & OK & Normal $\left(23^{\circ} \mathrm{C}\right)$ & Normal (47\%) & OK & OK & 139 & -89.058333 & 41.413333 \\
\hline 8/28/09 15:20 & $15: 23$ & 5703195 & Normal & OK & Normal $\left(23^{\circ} \mathrm{C}\right)$ & Normal (47\%) & OK & OK & 139 & -89.050833 & 41.460278 \\
\hline $8 / 28 / 09$ 15:25 & $15: 29$ & 5703195 & Normal & OK & Normal $\left(23^{\circ} \mathrm{C}\right)$ & Normal (47\%) & OK & OK & 139 & -89.058333 & 41.375833 \\
\hline 8/28/09 15:30 & $15: 35$ & 5703195 & Normal & OK & Normal $\left(23^{\circ} \mathrm{C}\right)$ & Normal (47\%) & OK & OK & 139 & -89.125833 & 41.368611 \\
\hline $8 / 28 / 09$ 15:35 & $15: 40$ & 5703195 & Normal & OK & Normal $\left(23^{\circ} \mathrm{C}\right)$ & Normal (47\%) & OK & OK & 139 & -89.125833 & 41.368611 \\
\hline 8/28/09 15:40 & $15: 46$ & 5703195 & Normal & OK & Normal $\left(23^{\circ} \mathrm{C}\right)$ & Normal (47\%) & OK & OK & 139 & -89.125833 & 41.368611 \\
\hline $8 / 28 / 09$ 15:45 & $15: 51$ & 5703195 & Normal & OK & Normal $\left(23^{\circ} \mathrm{C}\right)$ & Normal $(47 \%)$ & OK & OK & 139 & -89.200000 & 41.364444 \\
\hline $8 / 28 / 09$ 15:50 & $15: 57$ & 5703195 & Normal & OK & Normal $\left(23^{\circ} \mathrm{C}\right)$ & Normal $(47 \%)$ & OK & OK & 139 & -89.240556 & 41.371667 \\
\hline $8 / 28 / 09$ 16:00 & $16: 03$ & 5703195 & Normal & OK & Normal $\left(23^{\circ} \mathrm{C}\right)$ & Normal $(47 \%)$ & OK & OK & 139 & -89.458333 & 41.400833 \\
\hline $8 / 28 / 09$ 16:05 & $16: 13$ & 5703195 & Normal & OK & Normal $\left(23^{\circ} \mathrm{C}\right)$ & Normal $(47 \%)$ & OK & OK & 139 & -89.558333 & 41.393611 \\
\hline $8 / 28 / 09$ 16:11 & $16: 14$ & 5703195 & Normal & OK & Normal $\left(23^{\circ} \mathrm{C}\right)$ & Normal $(47 \%)$ & OK & OK & 139 & -89.558333 & 41.393611 \\
\hline 8/28/09 16:15 & $16: 19$ & 5703195 & Normal & OK & Normal $\left(23^{\circ} \mathrm{C}\right)$ & Normal $(47 \%)$ & OK & OK & 139 & -89.558333 & 41.393611 \\
\hline $8 / 28 / 09$ 16:20 & $16: 25$ & 5703195 & Normal & OK & Normal $\left(23^{\circ} \mathrm{C}\right)$ & Normal (47\%) & OK & OK & 139 & -89.558333 & 41.393611 \\
\hline $8 / 28 / 09$ 16:25 & $16: 30$ & 5703195 & Normal & OK & Normal $\left(23^{\circ} \mathrm{C}\right)$ & Normal (47\%) & OK & OK & 139 & -89.623889 & 41.394722 \\
\hline $8 / 28 / 09$ 16:30 & $16: 36$ & 5703195 & Normal & OK & Normal $\left(23^{\circ} \mathrm{C}\right)$ & Normal (47\%) & OK & OK & 139 & -89.725833 & 41.387500 \\
\hline $8 / 28 / 09$ 16:35 & $16: 41$ & 5703195 & Normal & OK & Normal $\left(23^{\circ} \mathrm{C}\right)$ & Normal $(47 \%)$ & OK & OK & 139 & -89.834167 & 41.388333 \\
\hline $8 / 28 / 0916: 40$ & $16: 47$ & 5703195 & Normal & OK & Normal $\left(23^{\circ} \mathrm{C}\right)$ & Normal $(47 \%)$ & OK & OK & 139 & -89.941667 & 41.416667 \\
\hline $8 / 28 / 09$ 16:45 & $16: 52$ & 5703195 & Normal & OK & Normal $\left(23^{\circ} \mathrm{C}\right)$ & Normal $(47 \%)$ & OK & OK & 139 & -90.050000 & 41.413333 \\
\hline $8 / 28 / 09$ 16:50 & $16: 52$ & 5703195 & Normal & OK & Normal $\left(23^{\circ} \mathrm{C}\right)$ & Normal $(47 \%)$ & OK & OK & 139 & -90.157222 & 41.430000 \\
\hline $8 / 28 / 09$ 16:55 & $16: 58$ & 5703195 & Normal & OK & Normal $\left(23^{\circ} \mathrm{C}\right)$ & Normal $(47 \%)$ & OK & OK & 139 & -90.267500 & 41.440556 \\
\hline $8 / 28 / 09$ 17:00 & 17:09 & 5703195 & Normal & OK & Normal $\left(23^{\circ} \mathrm{C}\right)$ & Normal $(47 \%)$ & OK & OK & 139 & -90.338333 & 41.476944 \\
\hline $8 / 28 / 09$ 17:05 & 17:09 & 5703195 & Normal & OK & Normal $\left(23^{\circ} \mathrm{C}\right)$ & Normal $(47 \%)$ & OK & OK & 139 & -90.334167 & 41.486389 \\
\hline $8 / 28 / 09$ 17:11 & $17: 15$ & 5703195 & Normal & OK & Normal $\left(23^{\circ} \mathrm{C}\right)$ & Normal $(47 \%)$ & OK & OK & 139 & -90.334167 & 41.486389 \\
\hline $8 / 28 / 09$ 17:15 & $17: 20$ & 5703195 & Normal & OK & Normal $\left(23^{\circ} \mathrm{C}\right)$ & Normal $(47 \%)$ & OK & OK & 139 & -90.334167 & 41.486389 \\
\hline $8 / 28 / 09$ 17:20 & $17: 26$ & 5703195 & Normal & OK & Normal $\left(23^{\circ} \mathrm{C}\right)$ & Normal $(47 \%)$ & OK & OK & 139 & -90.334167 & 41.486389 \\
\hline $8 / 28 / 09$ 17:25 & $17: 31$ & 5703195 & Normal & OK & Normal $\left(23^{\circ} \mathrm{C}\right)$ & Normal $(47 \%)$ & OK & OK & 139 & -90.340556 & 41.511389 \\
\hline 8/28/09 17:30 & $17: 37$ & 5703195 & Normal & OK & Normal $\left(23^{\circ} \mathrm{C}\right)$ & Normal (47\%) & OK & OK & 139 & -90.234167 & 41.562500 \\
\hline $8 / 28 / 09$ 17:35 & $17: 42$ & 5703195 & Normal & oK & Normal $\left(23^{\circ} \mathrm{C}\right)$ & Normal (47\%) & OK & OK & 139 & -90.209167 & 41.581111 \\
\hline $8 / 28 / 09$ 17:40 & $17: 42$ & 5703195 & Normal & OK & Normal $\left(23^{\circ} \mathrm{C}\right)$ & Normal $(47 \%)$ & OK & OK & 139 & -90.163333 & 41.642500 \\
\hline $8 / 28 / 09$ 17:45 & $17: 48$ & 5703195 & Normal & OK & Normal $\left(23^{\circ} \mathrm{C}\right)$ & Normal $(47 \%)$ & OK & OK & 139 & -90.080000 & 41.690556 \\
\hline $8 / 28 / 09$ 17:50 & $17: 53$ & 5703195 & Normal & OK & Normal $\left(23^{\circ} \mathrm{C}\right)$ & Normal $(47 \%)$ & OK & OK & 139 & -89.9833333 & 41.718611 \\
\hline $8 / 28 / 09$ 17:55 & $17: 59$ & 5703195 & Normal & OK & Normal $\left(25^{\circ} \mathrm{C}\right)$ & Normal (54\%) & OK & OK & 139 & -89.876944 & 41.744722 \\
\hline $8 / 28 / 09$ 18:00 & $18: 04$ & 5703195 & Normal & OK & Normal $\left(25^{\circ} \mathrm{C}\right)$ & Normal $(54 \%)$ & OK & OK & 139 & -89.775000 & 41.757222 \\
\hline 8/28/09 18:05 & $18: 10$ & 5703195 & Normal & OK & Normal $\left(25^{\circ} \mathrm{C}\right)$ & Normal (54\%) & OK & OK & 139 & -89.689444 & 41.750833 \\
\hline 8/28/09 18:08 & $18: 10$ & 5703195 & Alarm & OK & Normal $\left(25^{\circ} \mathrm{C}\right)$ & Normal (54\%) & Shocked & OK & 139 & -89.689444 & 41.762500 \\
\hline 8/28/09 18:10 & $18: 15$ & 5703195 & Alarm & OK & Normal $\left(25^{\circ} \mathrm{C}\right)$ & Normal $(54 \%)$ & Shocked & OK & 139 & -89.689444 & 41.762500 \\
\hline $8 / 28 / 09$ 18:15 & $18: 21$ & 5703195 & Normal & OK & Normal $\left(25^{\circ} \mathrm{C}\right)$ & Normal (54\%) & OK & OK & 139 & -89.678056 & 41.750833 \\
\hline $8 / 28 / 09$ 18:20 & $18: 26$ & 5703195 & Normal & OK & Normal $\left(25^{\circ} \mathrm{C}\right)$ & Normal (54\%) & OK & OK & 139 & -89.635278 & 41.759167 \\
\hline $8 / 28 / 0918: 25$ & $18: 32$ & 5703195 & Normal & OK & Normal $\left(25^{\circ} \mathrm{C}\right)$ & Normal (54\%) & OK & OK & 139 & -89.5322222 & 41.789444 \\
\hline 8/28/09 18:30 & $18: 38$ & 5703195 & Normal & OK & Normal $\left(25^{\circ} \mathrm{C}\right)$ & Normal (54\%) & OK & OK & 139 & -89.432222 & 41.822778 \\
\hline $8 / 28 / 09$ 18:35 & $18: 38$ & 5703195 & Normal & OK & Normal $\left(25^{\circ} \mathrm{C}\right)$ & Normal (54\%) & OK & OK & 139 & -89.336389 & 41.822778 \\
\hline $8 / 28 / 0918: 40$ & $18: 43$ & 5703195 & Normal & oK & Normal $\left(25^{\circ} \mathrm{C}\right)$ & Normal (54\%) & OK & OK & 139 & -89.231111 & 41.850833 \\
\hline $8 / 28 / 09$ 18:45 & $18: 48$ & 5703195 & Normal & oK & Normal $\left(26^{\circ} \mathrm{C}\right)$ & Normal (52\%) & OK & OK & 139 & -89.125000 & 41.883333 \\
\hline $8 / 28 / 0918: 50$ & $18: 54$ & 5703195 & Normal & OK & Normal $\left(26^{\circ} \mathrm{C}\right)$ & Normal $(52 \%)$ & OK & OK & 139 & -89.025000 & 41.904167 \\
\hline $8 / 28 / 09$ 18:55 & 19:00 & 5703195 & Normal & OK & Normal $\left(26^{\circ} \mathrm{C}\right)$ & Normal $(52 \%)$ & OK & OK & 139 & -88.913333 & 41.900000 \\
\hline $8 / 28 / 09$ 19:00 & 19:05 & 5703195 & Normal & OK & Normal $\left(26^{\circ} \mathrm{C}\right)$ & Normal $(52 \%)$ & OK & OK & 139 & -88.814444 & 41.900000 \\
\hline 8/28/09 19:05 & 19:11 & 5703195 & Normal & OK & Normal $\left(26^{\circ} \mathrm{C}\right)$ & Normal $(52 \%)$ & OK & OK & 139 & -88.739444 & 41.900000 \\
\hline 8/28/09 19:10 & $19: 16$ & 5703195 & Normal & OK & Normal $\left(26^{\circ} \mathrm{C}\right)$ & Normal $(52 \%)$ & OK & OK & 139 & -88.739444 & 41.900000 \\
\hline $8 / 28 / 09$ 19:15 & $19: 22$ & 5703195 & Normal & OK & Normal $\left(26^{\circ} \mathrm{C}\right)$ & Normal (50\%) & OK & OK & 139 & -88.737500 & 41.900000 \\
\hline $8 / 28 / 09$ 19:20 & 19:27 & 5703195 & Normal & OK & Normal $\left(26^{\circ} \mathrm{C}\right)$ & Normal $(50 \%)$ & OK & OK & 139 & -88.737500 & 41.900000 \\
\hline $8 / 28 / 09$ 19:25 & $19: 33$ & 5703195 & Normal & OK & Normal $\left(26^{\circ} \mathrm{C}\right)$ & Normal $(50 \%)$ & OK & OK & 139 & -88.639444 & 41.894722 \\
\hline 8/28/09 19:30 & 19:38 & 5703195 & Normal & OK & Normal $\left(26^{\circ} \mathrm{C}\right)$ & Normal $(50 \%)$ & OK & OK & 139 & -88.538333 & 41.859167 \\
\hline 8/28/09 19:35 & 19:38 & 5703195 & Normal & OK & Normal $\left(26^{\circ} \mathrm{C}\right)$ & Normal $(50 \%)$ & OK & OK & 139 & -88.448889 & 41.812500 \\
\hline 8/28/09 19:40 & $19: 44$ & 5703195 & Normal & OK & Normal $\left(26^{\circ} \mathrm{C}\right)$ & Normal $(50 \%)$ & OK & OK & 139 & -88.347778 & 41.794722 \\
\hline $8 / 28 / 09$ 19:45 & $19: 49$ & 5703195 & Normal & OK & Normal $\left(26^{\circ} \mathrm{C}\right)$ & Normal $(50 \%)$ & OK & OK & 139 & -88.261389 & 41.800000 \\
\hline 8/28/09 19:50 & 19:55 & 5703195 & Normal & OK & Normal $\left(26^{\circ} \mathrm{C}\right)$ & Normal $(50 \%)$ & OK & OK & 139 & -88.156111 & 41.806111 \\
\hline 8/28/09 19:55 & $20: 00$ & 5703195 & Normal & OK & Normal $\left(26^{\circ} \mathrm{C}\right)$ & Normal (50\%) & OK & OK & 139 & -88.062500 & 41.810278 \\
\hline 8/28/09 20:00 & 20:06 & 5703195 & Normal & OK & Normal $\left(26^{\circ} \mathrm{C}\right)$ & Normal (50\%) & OK & OK & 139 & -88.054167 & 41.807222 \\
\hline 8/28/09 20:05 & $20: 11$ & 5703195 & Normal & OK & Normal $\left(27^{\circ} \mathrm{C}\right)$ & Normal (52\%) & OK & OK & 139 & -88.054167 & 41.781111 \\
\hline $8 / 28 / 0920: 10$ & $20: 22$ & 5703195 & Normal & $\mathrm{OK}$ & Normal $\left(27^{\circ} \mathrm{C}\right)$ & Normal $(52 \%)$ & OK & OK & 139 & -88.035278 & 41.732222 \\
\hline $8 / 28 / 0913: 40$ & $13: 44$ & 5703196 & Normal & OK & Normal $\left(23^{\circ} \mathrm{C}\right)$ & Normal (54\%) & OK & $\overline{O K}$ & 138 & -87.971667 & 41.732222 \\
\hline $8 / 28 / 09$ 13:45 & $13: 49$ & 5703196 & Normal & OK & Normal $\left(23^{\circ} \mathrm{C}\right)$ & Normal (54\%) & OK & OK & 138 & -87.971667 & 41.732222 \\
\hline 8/28/09 13:50 & $14: 00$ & 5703196 & Normal & OK & Normal $\left(23^{\circ} \mathrm{C}\right)$ & Normal (54\%) & OK & OK & 138 & -87.971667 & 41.732222 \\
\hline 8/28/09 13:55 & $14: 00$ & 5703196 & Normal & OK & Normal $\left(23^{\circ} \mathrm{C}\right)$ & Normal (54\%) & OK & OK & 138 & -87.971667 & 41.732222 \\
\hline $8 / 28 / 09$ 14:00 & $14: 06$ & 5703196 & Normal & OK & Normal $\left(23^{\circ} \mathrm{C}\right)$ & Normal (54\%) & OK & OK & 138 & -88.068611 & 41.684167 \\
\hline $8 / 28 / 09$ 14:05 & $14: 11$ & 5703196 & Normal & OK & Normal $\left(23^{\circ} \mathrm{C}\right)$ & Normal (54\%) & OK & OK & 138 & -88.154167 & 41.640556 \\
\hline $8 / 28 / 09$ 14:10 & $14: 17$ & 5703196 & Normal & OK & Normal $\left(23^{\circ} \mathrm{C}\right)$ & Normal (54\%) & OK & OK & 138 & -88.181111 & 41.568611 \\
\hline $8 / 28 / 09$ 14:15 & $14: 22$ & 5703196 & Normal & OK & Normal $\left(23^{\circ} \mathrm{C}\right)$ & Normal (54\%) & OK & OK & 138 & -88.197778 & 41.493611 \\
\hline $8 / 28 / 0914: 20$ & $14: 22$ & 5703196 & Normal & OK & Normal $\left(23^{\circ} \mathrm{C}\right)$ & Normal (54\%) & OK & OK & 138 & -88.280000 & 41.462500 \\
\hline $8 / 28 / 0914: 25$ & $14: 28$ & 5703196 & Normal & OK & Normal $\left(23^{\circ} \mathrm{C}\right)$ & Normal (54\%) & OK & OK & 138 & -88.371667 & 41.417500 \\
\hline $8 / 28 / 09$ 14:32 & $14: 39$ & 5703196 & Normal & OK & Normal $\left(23^{\circ} \mathrm{C}\right)$ & Normal (54\%) & OK & OK & 138 & -88.422778 & 41.392500 \\
\hline $8 / 28 / 09$ 14:35 & $14: 39$ & 5703196 & Normal & OK & Normal $\left(23^{\circ} \mathrm{C}\right)$ & Normal (54\%) & OK & OK & 138 & -88.422778 & 41.392500 \\
\hline $8 / 28 / 0914: 40$ & $14: 44$ & 5703196 & Normal & OK & Normal $\left(23^{\circ} \mathrm{C}\right)$ & Normal (54\%) & OK & OK & 138 & -88.422778 & 41.392500 \\
\hline $8 / 28 / 09$ 14:45 & $14: 50$ & 5703196 & Normal & OK & Normal $\left(23^{\circ} \mathrm{C}\right)$ & Normal (54\%) & OK & OK & 138 & -88.461389 & 41.378056 \\
\hline $8 / 28 / 09$ 14:50 & $14: 56$ & 5703196 & Normal & OK & Normal $\left(23^{\circ} \mathrm{C}\right)$ & Normal (54\%) & OK & OK & 138 & -88.575000 & 41.376944 \\
\hline $8 / 28 / 09$ 14:55 & $15: 01$ & 5703196 & Normal & OK & Normal $\left(24^{\circ} \mathrm{C}\right)$ & Normal (54\%) & OK & OK & 138 & -88.717500 & 41.375833 \\
\hline $8 / 28 / 0915: 00$ & $15: 07$ & 5703196 & Normal & OK & Normal $\left(24^{\circ} \mathrm{C}\right)$ & Normal ( $54 \%)$ & OK & OK & 138 & -88.792500 & 41.376944 \\
\hline
\end{tabular}




\begin{tabular}{|c|c|c|c|c|c|c|c|c|c|c|c|}
\hline Event Time & at QC Hub & Tag ID & Tag Status & Seal & Temperature & Humidity & Shock & Battery & RSSI & Longitude & Latitude \\
\hline $8 / 28 / 0915: 05$ & $15: 12$ & 5703196 & Normal & OK & Normal $\left(24^{\circ} \mathrm{C}\right)$ & Normal (54\%) & OK & OK & 138 & -88.901944 & 41.368611 \\
\hline $8 / 28 / 09$ 15:10 & $15: 12$ & 5703196 & Normal & OK & Normal $\left(24^{\circ} \mathrm{C}\right)$ & Normal (54\%) & OK & OK & 138 & -89.014444 & 41.367500 \\
\hline $8 / 28 / 0915: 15$ & $15: 18$ & 5703196 & Normal & OK & Normal $\left(24^{\circ} \mathrm{C}\right)$ & Normal (54\%) & $\mathrm{OK}$ & $\mathrm{OK}$ & 138 & -89.058333 & 41.413333 \\
\hline $8 / 28 / 0915: 20$ & $15: 23$ & 5703196 & Normal & OK & Normal $\left(24^{\circ} \mathrm{C}\right)$ & Normal (54\%) & $\mathrm{OK}$ & OK & 138 & -89.050833 & 41.460278 \\
\hline $8 / 28 / 09$ 15:25 & $15: 29$ & 5703196 & Normal & $\mathrm{OK}$ & Normal $\left(24^{\circ} \mathrm{C}\right)$ & Normal (54\%) & OK & OK & 138 & -89.058333 & 41.375833 \\
\hline $8 / 28 / 09$ 15:30 & $15: 35$ & 5703196 & Normal & OK & Normal $\left(24^{\circ} \mathrm{C}\right)$ & Normal (54\%) & $\mathrm{OK}$ & OK & 138 & -89.125833 & 41.368611 \\
\hline $8 / 28 / 09$ 15:35 & $15: 40$ & 5703196 & Normal & OK & Normal $\left(24^{\circ} \mathrm{C}\right)$ & Normal (54\%) & $\mathrm{OK}$ & OK & 138 & -89.125833 & 41.368611 \\
\hline $8 / 28 / 0915: 40$ & $15: 46$ & 5703196 & Normal & OK & Normal $\left(24^{\circ} \mathrm{C}\right)$ & Normal (54\%) & OK & OK & 138 & -89.125833 & 41.368611 \\
\hline 8/28/09 15:45 & $15: 51$ & 5703196 & Normal & OK & Normal $\left(24^{\circ} \mathrm{C}\right)$ & Normal (54\%) & OK & OK & 138 & -89.200000 & 41.364444 \\
\hline 8/28/09 15:50 & $15: 57$ & 5703196 & Normal & OK & Normal $\left(24^{\circ} \mathrm{C}\right)$ & Normal (54\%) & OK & OK & 138 & -89.240556 & 41.371667 \\
\hline $8 / 28 / 0916: 00$ & $16: 03$ & 5703196 & Normal & OK & Normal $\left(24^{\circ} \mathrm{C}\right)$ & Normal (54\%) & OK & OK & 138 & -89.458333 & 41.400833 \\
\hline 8/28/09 16:05 & $16: 13$ & 5703196 & Normal & OK & Normal $\left(24^{\circ} \mathrm{C}\right)$ & Normal (54\%) & $\mathrm{OK}$ & OK & 138 & -89.558333 & 41.393611 \\
\hline 8/28/09 16:11 & $16: 14$ & 5703196 & Normal & OK & Normal $\left(24^{\circ} \mathrm{C}\right)$ & Normal (54\%) & OK & OK & 138 & -89.558333 & 41.393611 \\
\hline 8/28/09 16:15 & $16: 19$ & 5703196 & Normal & OK & Normal $\left(24^{\circ} \mathrm{C}\right)$ & Normal (54\%) & OK & OK & 138 & -89.558333 & 41.393611 \\
\hline $8 / 28 / 09$ 16:20 & $16: 25$ & 5703196 & Normal & OK & Normal $\left(24^{\circ} \mathrm{C}\right)$ & Normal (54\%) & OK & OK & 138 & -89.558333 & 41.393611 \\
\hline 8/28/09 16:25 & $16: 30$ & 5703196 & Normal & OK & Normal $\left(24^{\circ} \mathrm{C}\right)$ & Normal (54\%) & OK & OK & 138 & -89.623889 & 41.394722 \\
\hline $8 / 28 / 09$ 16:30 & $16: 36$ & 5703196 & Normal & OK & Normal $\left(24^{\circ} \mathrm{C}\right)$ & Normal (54\%) & OK & OK & 138 & -89.725833 & 41.387500 \\
\hline 8/28/09 16:35 & $16: 41$ & 5703196 & Normal & OK & Normal $\left(24^{\circ} \mathrm{C}\right)$ & Normal (54\%) & $\mathrm{OK}$ & OK & 138 & -89.834167 & 41.388333 \\
\hline 8/28/09 16:40 & $16: 47$ & 5703196 & Normal & OK & Normal $\left(24^{\circ} \mathrm{C}\right)$ & Normal (54\%) & OK & OK & 138 & -89.941667 & 41.416667 \\
\hline $8 / 28 / 0916: 45$ & $16: 52$ & 5703196 & Normal & OK & Normal $\left(24^{\circ} \mathrm{C}\right)$ & Normal (54\%) & OK & OK & 138 & -90.050000 & 41.413333 \\
\hline 8/28/09 16:50 & $16: 52$ & 5703196 & Normal & OK & Normal $\left(24^{\circ} \mathrm{C}\right)$ & Normal (54\%) & OK & OK & 138 & -90.157222 & 41.430000 \\
\hline $8 / 28 / 0916: 55$ & $16: 58$ & 5703196 & Normal & OK & Normal $\left(24^{\circ} \mathrm{C}\right)$ & Normal (54\%) & OK & OK & 138 & -90.267500 & 41.440556 \\
\hline 8/28/09 17:00 & $17: 09$ & 5703196 & Normal & OK & Normal $\left(24^{\circ} \mathrm{C}\right)$ & Normal (54\%) & OK & OK & 138 & -90.338333 & 41.476944 \\
\hline 8/28/09 17:05 & $17: 09$ & 5703196 & Normal & OK & Normal $\left(24^{\circ} \mathrm{C}\right)$ & Normal (54\%) & OK & OK & 138 & -90.334167 & 41.486389 \\
\hline 8/28/09 17:11 & $17: 15$ & 5703196 & Normal & OK & Normal $\left(24^{\circ} \mathrm{C}\right)$ & Normal (54\%) & OK & OK & 138 & -90.334167 & 41.486389 \\
\hline $8 / 28 / 0917: 15$ & $17: 20$ & 5703196 & Normal & OK & Normal $\left(24^{\circ} \mathrm{C}\right)$ & Normal (54\%) & OK & OK & 138 & -90.334167 & 41.486389 \\
\hline $8 / 28 / 0917: 20$ & $17: 26$ & 5703196 & Normal & OK & Normal $\left(24^{\circ} \mathrm{C}\right)$ & Normal (54\%) & OK & OK & 138 & -90.334167 & 41.486389 \\
\hline $8 / 28 / 0917: 25$ & $17: 31$ & 5703196 & Normal & OK & Normal $\left(24^{\circ} \mathrm{C}\right)$ & Normal (54\%) & OK & OK & 138 & -90.340556 & 41.511389 \\
\hline $8 / 28 / 0917: 30$ & $17: 37$ & 5703196 & Normal & OK & Normal $\left(24^{\circ} \mathrm{C}\right)$ & Normal (54\%) & OK & OK & 138 & -90.234167 & 41.562500 \\
\hline $8 / 28 / 0917: 35$ & $17: 42$ & 5703196 & Normal & OK & Normal $\left(24^{\circ} \mathrm{C}\right)$ & Normal (54\%) & OK & OK & 138 & -90.209167 & 41.581111 \\
\hline $8 / 28 / 09$ 17:40 & $17: 42$ & 5703196 & Normal & OK & Normal $\left(24^{\circ} \mathrm{C}\right)$ & Normal $(54 \%)$ & OK & OK & 138 & -90.163333 & 41.642500 \\
\hline $8 / 28 / 09$ 17:45 & $17: 48$ & 5703196 & Normal & OK & Normal $\left(24^{\circ} \mathrm{C}\right)$ & Normal $(54 \%)$ & OK & OK & 138 & -90.080000 & 41.690556 \\
\hline $8 / 28 / 0917: 50$ & $17: 53$ & 5703196 & Normal & OK & Normal $\left(24^{\circ} \mathrm{C}\right)$ & Normal $(54 \%)$ & OK & OK & 138 & -89.983333 & 41.718611 \\
\hline $8 / 28 / 0917: 55$ & $17: 59$ & 5703196 & Normal & OK & Normal $\left(27^{\circ} \mathrm{C}\right)$ & Normal (51\%) & OK & OK & 138 & -89.876944 & 41.744722 \\
\hline $8 / 28 / 0918: 00$ & $18: 04$ & 5703196 & Normal & OK & Normal $\left(27^{\circ} \mathrm{C}\right)$ & Normal (51\%) & OK & OK & 138 & -89.775000 & 41.757222 \\
\hline $8 / 28 / 0918: 05$ & $18: 10$ & 5703196 & Normal & OK & Normal $\left(27^{\circ} \mathrm{C}\right)$ & Normal (51\%) & OK & OK & 138 & -89.689444 & 41.750833 \\
\hline 8/28/09 18:10 & $18: 15$ & 5703196 & Normal & OK & Normal $\left(27^{\circ} \mathrm{C}\right)$ & Normal (51\%) & OK & OK & 138 & -89.689444 & 41.762500 \\
\hline $8 / 28 / 09$ 18:15 & $18: 21$ & 5703196 & Normal & OK & Normal $\left(27^{\circ} \mathrm{C}\right)$ & Normal (51\%) & OK & OK & 138 & -89.678056 & 41.750833 \\
\hline $8 / 28 / 0918: 20$ & $18: 26$ & 5703196 & Normal & OK & Normal $\left(27^{\circ} \mathrm{C}\right)$ & Normal (51\%) & $\mathrm{OK}$ & OK & 138 & -89.635278 & 41.759167 \\
\hline $8 / 28 / 0918: 25$ & $18: 32$ & 5703196 & Normal & OK & Normal $\left(27^{\circ} \mathrm{C}\right)$ & Normal (51\%) & OK & OK & 138 & -89.532222 & 41.789444 \\
\hline $8 / 28 / 0918: 30$ & $18: 38$ & 5703196 & Normal & OK & Normal $\left(27^{\circ} \mathrm{C}\right)$ & Normal (51\%) & OK & OK & 138 & -89.432222 & 41.822778 \\
\hline $8 / 28 / 0918: 35$ & $18: 38$ & 5703196 & Normal & OK & Normal $\left(27^{\circ} \mathrm{C}\right)$ & Normal (51\%) & OK & ok & 138 & -89.336389 & 41.822778 \\
\hline $8 / 28 / 0918: 40$ & $18: 43$ & 5703196 & Normal & OK & Normal $\left(27^{\circ} \mathrm{C}\right)$ & Normal (51\%) & OK & OK & 138 & -89.231111 & 41.850833 \\
\hline $8 / 28 / 0918: 45$ & $18: 48$ & 5703196 & Normal & OK & Normal $\left(28^{\circ} \mathrm{C}\right)$ & Normal $(49 \%)$ & OK & OK & 138 & -89.125000 & 41.883333 \\
\hline $8 / 28 / 09$ 18:50 & $18: 54$ & 5703196 & Normal & OK & Normal $\left(28^{\circ} \mathrm{C}\right)$ & Normal (49\%) & OK & OK & 138 & -89.025000 & 41.904167 \\
\hline 8/28/09 18:55 & $19: 00$ & 5703196 & Normal & OK & Normal $\left(28^{\circ} \mathrm{C}\right)$ & Normal $(49 \%)$ & OK & OK & 138 & -88.913333 & 41.900000 \\
\hline 8/28/09 19:00 & $19: 05$ & 5703196 & Normal & OK & Normal $\left(28^{\circ} \mathrm{C}\right)$ & Normal $(49 \%)$ & OK & OK & 138 & -88.814444 & 41.900000 \\
\hline 8/28/09 19:05 & $19: 11$ & 5703196 & Normal & OK & Normal $\left(28^{\circ} \mathrm{C}\right)$ & Normal $(49 \%)$ & OK & OK & 138 & -88.739444 & 41.900000 \\
\hline 8/28/09 19:10 & $19: 16$ & 5703196 & Normal & OK & Normal $\left(28^{\circ} \mathrm{C}\right)$ & Normal $(49 \%)$ & OK & OK & 138 & -88.739444 & 41.900000 \\
\hline 8/28/09 19:15 & $19: 22$ & 5703196 & Normal & OK & Normal $\left(28^{\circ} \mathrm{C}\right)$ & Normal $(49 \%)$ & OK & OK & 138 & -88.737500 & 41.900000 \\
\hline $8 / 28 / 09$ 19:20 & $19: 27$ & 5703196 & Normal & OK & Normal $\left(28^{\circ} \mathrm{C}\right)$ & Normal $(49 \%)$ & OK & OK & 138 & -88.737500 & 41.900000 \\
\hline $8 / 28 / 09$ 19:25 & $19: 33$ & 5703196 & Normal & OK & Normal $\left(28^{\circ} \mathrm{C}\right)$ & Normal $(49 \%)$ & OK & OK & 138 & -88.639444 & 41.894722 \\
\hline $8 / 28 / 0919: 30$ & $19: 38$ & 5703196 & Normal & OK & Normal $\left(28^{\circ} \mathrm{C}\right)$ & Normal $(49 \%)$ & OK & OK & 138 & -88.538333 & 41.859167 \\
\hline 8/28/09 19:35 & $19: 38$ & 5703196 & Normal & OK & Normal $\left(28^{\circ} \mathrm{C}\right)$ & Normal $(49 \%)$ & OK & OK & 138 & -88.448889 & 41.812500 \\
\hline $8 / 28 / 09$ 19:40 & $19: 44$ & 5703196 & Normal & OK & Normal $\left(28^{\circ} \mathrm{C}\right)$ & Normal $(49 \%)$ & OK & OK & 138 & -88.347778 & 41.794722 \\
\hline $8 / 28 / 09$ 19:45 & $19: 49$ & 5703196 & Normal & OK & Normal $\left(28^{\circ} \mathrm{C}\right)$ & Normal (49\%) & OK & ok & 138 & -88.261389 & 41.800000 \\
\hline $8 / 28 / 09$ 19:50 & $19: 55$ & 5703196 & Normal & OK & Normal $\left(28^{\circ} \mathrm{C}\right)$ & Normal $(49 \%)$ & OK & OK & 138 & -88.156111 & 41.806111 \\
\hline $8 / 28 / 09$ 19:55 & $20: 00$ & 5703196 & Normal & OK & Normal $\left(28^{\circ} \mathrm{C}\right)$ & Normal $(49 \%)$ & OK & OK & 138 & -88.062500 & 41.810278 \\
\hline $8 / 28 / 0920: 00$ & 20:06 & 5703196 & Normal & OK & Normal $\left(28^{\circ} \mathrm{C}\right)$ & Normal $(49 \%)$ & OK & OK & 138 & -88.054167 & 41.807222 \\
\hline $8 / 28 / 0920: 05$ & $20: 11$ & 5703196 & Normal & OK & Normal $\left(28^{\circ} \mathrm{C}\right)$ & Normal $(49 \%)$ & OK & OK & 138 & -88.054167 & 41.781111 \\
\hline 8/28/09 20:10 & $20: 22$ & 5703196 & Normal & OK & Normal $\left(28^{\circ} \mathrm{C}\right)$ & Normal (49\%) & OK & OK & 138 & -88.035278 & 41.732222 \\
\hline $8 / 28 / 0913: 40$ & $13: 44$ & 5703197 & Normal & OK & Normal $\left(23^{\circ} \mathrm{C}\right)$ & Normal (56\%) & OK & OK & 140 & -87.971667 & 41.732222 \\
\hline $8 / 28 / 0913: 45$ & $13: 49$ & 5703197 & Normal & OK & Normal $\left(23^{\circ} \mathrm{C}\right)$ & Normal (56\%) & OK & OK & 140 & -87.971667 & 41.732222 \\
\hline $8 / 28 / 09$ 13:50 & $14: 00$ & 5703197 & Normal & OK & Normal $\left(23^{\circ} \mathrm{C}\right)$ & Normal (56\%) & OK & OK & 140 & -87.971667 & 41.732222 \\
\hline $8 / 28 / 0913: 55$ & $14: 00$ & 5703197 & Normal & OK & Normal $\left(23^{\circ} \mathrm{C}\right)$ & Normal (56\%) & OK & OK & 140 & -87.971667 & 41.732222 \\
\hline $8 / 28 / 0914: 00$ & $14: 06$ & 5703197 & Normal & OK & Normal $\left(23^{\circ} \mathrm{C}\right)$ & Normal (56\%) & OK & OK & 140 & -88.068611 & 41.684167 \\
\hline $8 / 28 / 09$ 14:05 & $14: 11$ & 5703197 & Normal & OK & Normal $\left(23^{\circ} \mathrm{C}\right)$ & Normal $(56 \%)$ & OK & OK & 140 & -88.154167 & 41.640556 \\
\hline 8/28/09 14:10 & $14: 17$ & 5703197 & Normal & OK & Normal $\left(23^{\circ} \mathrm{C}\right)$ & Normal $(56 \%)$ & OK & OK & 140 & -88.181111 & 41.568611 \\
\hline $8 / 28 / 09$ 14:15 & $14: 22$ & 5703197 & Normal & OK & Normal $\left(23^{\circ} \mathrm{C}\right)$ & Normal (56\%) & OK & OK & 140 & -88.197778 & 41.493611 \\
\hline $8 / 28 / 0914: 20$ & $14: 22$ & 5703197 & Normal & OK & Normal $\left(23^{\circ} \mathrm{C}\right)$ & Normal $(56 \%)$ & $\mathrm{OK}$ & OK & 140 & -88.280000 & 41.462500 \\
\hline $8 / 28 / 0914: 25$ & $14: 28$ & 5703197 & Normal & OK & Normal $\left(23^{\circ} \mathrm{C}\right)$ & Normal (56\%) & OK & OK & 140 & -88.371667 & 41.417500 \\
\hline $8 / 28 / 0914: 32$ & $14: 39$ & 5703197 & Normal & OK & Normal $\left(23^{\circ} \mathrm{C}\right)$ & Normal $(56 \%)$ & OK & OK & 140 & -88.422778 & 41.392500 \\
\hline $8 / 28 / 0914: 35$ & $14: 39$ & 5703197 & Normal & OK & Normal $\left(23^{\circ} \mathrm{C}\right)$ & Normal (56\%) & OK & OK & 140 & -88.422778 & 41.392500 \\
\hline $8 / 28 / 0914: 40$ & $14: 44$ & 5703197 & Normal & OK & Normal $\left(23^{\circ} \mathrm{C}\right)$ & Normal (56\%) & OK & OK & 140 & -88.422778 & 41.392500 \\
\hline $8 / 28 / 0914: 45$ & $14: 50$ & 5703197 & Normal & OK & Normal $\left(23^{\circ} \mathrm{C}\right)$ & Normal $(56 \%)$ & OK & ok & 140 & -88.461389 & 41.378056 \\
\hline $8 / 28 / 0914: 50$ & $14: 56$ & 5703197 & Normal & OK & Normal $\left(23^{\circ} \mathrm{C}\right)$ & Normal (56\%) & OK & OK & 140 & -88.575000 & 41.376944 \\
\hline $8 / 28 / 09$ 14:55 & $15: 01$ & 5703197 & Normal & OK & Normal $\left(24^{\circ} \mathrm{C}\right)$ & Normal (54\%) & OK & OK & 140 & -88.717500 & 41.375833 \\
\hline $8 / 28 / 09$ 15:00 & $15: 07$ & 5703197 & Normal & OK & Normal $\left(24^{\circ} \mathrm{C}\right)$ & Normal (54\%) & OK & OK & 140 & -88.792500 & 41.376944 \\
\hline $8 / 28 / 09$ 15:05 & $15: 12$ & 5703197 & Normal & OK & Normal $\left(24^{\circ} \mathrm{C}\right)$ & Normal (54\%) & OK & OK & 140 & -88.901944 & 41.368611 \\
\hline $8 / 28 / 09$ 15:10 & $15: 12$ & 5703197 & Normal & OK & Normal $\left(24^{\circ} \mathrm{C}\right)$ & Normal (54\%) & OK & OK & 140 & -89.014444 & 41.367500 \\
\hline $8 / 28 / 09$ 15:15 & $15: 18$ & 5703197 & Normal & OK & Normal $\left(24^{\circ} \mathrm{C}\right)$ & Normal (54\%) & OK & OK & 140 & -89.058333 & 41.413333 \\
\hline
\end{tabular}




\begin{tabular}{|c|c|c|c|c|c|c|c|c|c|c|c|}
\hline Event Time & at QC Hub & Tag ID & Tag Status & Seal & Temperature & Humidity & Shock & Battery & RSSI & Longitude & Latitude \\
\hline $8 / 28 / 09$ 15:20 & $15: 23$ & 5703197 & Normal & OK & Normal $\left(24^{\circ} \mathrm{C}\right)$ & Normal (54\%) & OK & OK & 140 & -89.050833 & 41.460278 \\
\hline $8 / 28 / 09$ 15:25 & $15: 29$ & 5703197 & Normal & OK & Normal $\left(24^{\circ} \mathrm{C}\right)$ & Normal (54\%) & OK & OK & 140 & -89.058333 & 41.375833 \\
\hline $8 / 28 / 09$ 15:30 & $15: 35$ & 5703197 & Normal & OK & Normal $\left(24^{\circ} \mathrm{C}\right)$ & Normal (54\%) & OK & OK & 140 & -89.125833 & 41.368611 \\
\hline $8 / 28 / 09$ 15:35 & $15: 40$ & 5703197 & Normal & OK & Normal $\left(24^{\circ} \mathrm{C}\right)$ & Normal $(54 \%)$ & OK & OK & 140 & -89.125833 & 41.368611 \\
\hline $8 / 28 / 09$ 15:40 & $15: 46$ & 5703197 & Normal & OK & Normal $\left(24^{\circ} \mathrm{C}\right)$ & Normal (54\%) & OK & OK & 140 & -89.125833 & 41.368611 \\
\hline $8 / 28 / 09$ 15:45 & $15: 51$ & 5703197 & Normal & OK & Normal $\left(24^{\circ} \mathrm{C}\right)$ & Normal (54\%) & OK & OK & 140 & -89.200000 & 41.364444 \\
\hline $8 / 28 / 09$ 15:50 & $15: 57$ & 5703197 & Normal & OK & Normal $\left(24^{\circ} \mathrm{C}\right)$ & Normal (54\%) & OK & OK & 140 & -89.240556 & 41.371667 \\
\hline $8 / 28 / 0916: 00$ & $16: 03$ & 5703197 & Normal & OK & $\operatorname{Normal}\left(24^{\circ} \mathrm{C}\right)$ & Normal $(54 \%)$ & OK & OK & 140 & -89.458333 & 41.400833 \\
\hline $8 / 28 / 09$ 16:05 & $16: 13$ & 5703197 & Normal & OK & Normal $\left(24^{\circ} \mathrm{C}\right)$ & Normal (54\%) & OK & OK & 140 & -89.558333 & 41.393611 \\
\hline 8/28/09 16:11 & $16: 14$ & 5703197 & Normal & OK & $\operatorname{Normal}\left(24^{\circ} \mathrm{C}\right)$ & Normal (54\%) & OK & OK & 140 & -89.558333 & 41.393611 \\
\hline $8 / 28 / 0916: 15$ & $16: 19$ & 5703197 & Normal & OK & $\operatorname{Normal}\left(24^{\circ} \mathrm{C}\right)$ & Normal $(54 \%)$ & OK & OK & 140 & -89.558333 & 41.393611 \\
\hline $8 / 28 / 0916: 20$ & $16: 25$ & 5703197 & Normal & OK & $\operatorname{Normal}\left(24^{\circ} \mathrm{C}\right)$ & Normal (54\%) & OK & OK & 140 & -89.558333 & 41.393611 \\
\hline $8 / 28 / 0916: 25$ & $16: 30$ & 5703197 & Normal & OK & $\operatorname{Normal}\left(24^{\circ} \mathrm{C}\right)$ & Normal $(54 \%)$ & OK & OK & 140 & -89.623889 & 41.394722 \\
\hline $8 / 28 / 0916: 30$ & $16: 36$ & 5703197 & Normal & OK & $\operatorname{Normal}\left(24^{\circ} \mathrm{C}\right)$ & Normal $(54 \%)$ & OK & OK & 140 & -89.725833 & 41.387500 \\
\hline $8 / 28 / 0916: 35$ & $16: 41$ & 5703197 & Normal & OK & $\operatorname{Normal}\left(24^{\circ} \mathrm{C}\right)$ & Normal $(54 \%)$ & OK & OK & 140 & -89.834167 & 41.388333 \\
\hline $8 / 28 / 09$ 16:40 & $16: 47$ & 5703197 & Normal & $\mathrm{OK}$ & Normal $\left(24^{\circ} \mathrm{C}\right)$ & Normal (54\%) & OK & OK & 140 & -89.941667 & 41.416667 \\
\hline $8 / 28 / 09$ 16:45 & $16: 52$ & 5703197 & Normal & OK & Normal $\left(24^{\circ} \mathrm{C}\right)$ & Normal (54\%) & OK & OK & 140 & -90.050000 & 41.413333 \\
\hline 8/28/09 16:50 & $16: 52$ & 5703197 & Normal & OK & Normal $\left(24^{\circ} \mathrm{C}\right)$ & Normal (54\%) & OK & OK & 140 & -90.157222 & 41.430000 \\
\hline 8/28/09 16:55 & $16: 58$ & 5703197 & Normal & OK & Normal $\left(24^{\circ} \mathrm{C}\right)$ & Normal (54\%) & OK & OK & 140 & -90.267500 & 41.440556 \\
\hline 8/28/09 17:00 & 17:09 & 5703197 & Normal & OK & Normal $\left(24^{\circ} \mathrm{C}\right)$ & Normal (54\%) & OK & OK & 140 & -90.338333 & 41.476944 \\
\hline 8/28/09 17:05 & 17:09 & 5703197 & Normal & OK & Normal $\left(24^{\circ} \mathrm{C}\right)$ & Normal (54\%) & OK & OK & 140 & -90.334167 & 41.486389 \\
\hline 8/28/09 17:11 & $17: 15$ & 5703197 & Normal & OK & Normal $\left(24^{\circ} \mathrm{C}\right)$ & Normal (54\%) & OK & OK & 140 & -90.334167 & 41.486389 \\
\hline $8 / 28 / 09$ 17:15 & $17: 20$ & 5703197 & Normal & OK & Normal $\left(24^{\circ} \mathrm{C}\right)$ & Normal (54\%) & OK & OK & 140 & -90.334167 & 41.486389 \\
\hline $8 / 28 / 0917: 20$ & $17: 26$ & 5703197 & Normal & OK & $\operatorname{Normal}\left(24^{\circ} \mathrm{C}\right)$ & Normal $(54 \%)$ & OK & OK & 140 & -90.334167 & 41.486389 \\
\hline $8 / 28 / 0917: 25$ & $17: 31$ & 5703197 & Normal & OK & $\operatorname{Normal}\left(24^{\circ} \mathrm{C}\right)$ & Normal $(54 \%)$ & OK & OK & 140 & -90.340556 & 41.511389 \\
\hline $8 / 28 / 0917: 30$ & $17: 37$ & 5703197 & Normal & OK & $\operatorname{Normal}\left(24^{\circ} \mathrm{C}\right)$ & Normal $(54 \%)$ & OK & OK & 140 & -90.234167 & 41.562500 \\
\hline $8 / 28 / 0917: 35$ & $17: 42$ & 5703197 & Normal & OK & $\operatorname{Normal}\left(24^{\circ} \mathrm{C}\right)$ & Normal $(54 \%)$ & OK & OK & 140 & -90.209167 & 41.581111 \\
\hline $8 / 28 / 0917: 40$ & $17: 42$ & 5703197 & Normal & OK & $\operatorname{Normal}\left(24^{\circ} \mathrm{C}\right)$ & Normal $(54 \%)$ & ok & OK & 140 & -90.1633333 & 41.642500 \\
\hline $8 / 28 / 09$ 17:45 & $17: 48$ & 5703197 & Normal & OK & $\operatorname{Normal}\left(24^{\circ} \mathrm{C}\right)$ & Normal $(54 \%)$ & OK & OK & 140 & -90.080000 & 41.690556 \\
\hline $8 / 28 / 0917: 50$ & $17: 53$ & 5703197 & Normal & OK & $\operatorname{Normal}\left(24^{\circ} \mathrm{C}\right)$ & Normal $(54 \%)$ & OK & OK & 140 & -89.983333 & 41.718611 \\
\hline 8/28/09 17:55 & $17: 59$ & 5703197 & Normal & OK & Normal $\left(26^{\circ} \mathrm{C}\right)$ & Normal $(54 \%)$ & OK & OK & 140 & -89.876944 & 41.744722 \\
\hline $8 / 28 / 0918: 00$ & $18: 04$ & 5703197 & Normal & OK & Normal $\left(26^{\circ} \mathrm{C}\right)$ & Normal $(54 \%)$ & OK & OK & 140 & -89.775000 & 41.757222 \\
\hline 8/28/09 18:05 & $18: 10$ & 5703197 & Normal & OK & $\operatorname{Normal}\left(26^{\circ} \mathrm{C}\right)$ & Normal $(54 \%)$ & OK & OK & 140 & -89.689444 & 41.750833 \\
\hline 8/28/09 18:10 & $18: 15$ & 5703197 & Normal & OK & Normal $\left(26^{\circ} \mathrm{C}\right)$ & Normal $(54 \%)$ & OK & OK & 140 & -89.689444 & 41.762500 \\
\hline $8 / 28 / 0918: 15$ & $18: 21$ & 5703197 & Normal & OK & $\operatorname{Normal}\left(26^{\circ} \mathrm{C}\right)$ & Normal $(54 \%)$ & ok & OK & 140 & -89.678056 & 41.750833 \\
\hline $8 / 28 / 0918: 20$ & $18: 26$ & 5703197 & Normal & OK & $\operatorname{Normal}\left(26^{\circ} \mathrm{C}\right)$ & Normal $(54 \%)$ & OK & OK & 140 & -89.635278 & 41.759167 \\
\hline $8 / 28 / 09$ 18:25 & $18: 32$ & 5703197 & Normal & OK & Normal $\left(26^{\circ} \mathrm{C}\right)$ & Normal (54\%) & OK & OK & 140 & -89.532222 & 41.789444 \\
\hline $8 / 28 / 0918: 30$ & $18: 38$ & 5703197 & Normal & OK & Normal $\left(26^{\circ} \mathrm{C}\right)$ & Normal (54\%) & OK & OK & 140 & -89.432222 & 41.822778 \\
\hline $8 / 28 / 09$ 18:35 & $18: 38$ & 5703197 & Normal & OK & Normal $\left(26^{\circ} \mathrm{C}\right)$ & Normal (54\%) & OK & OK & 140 & -89.336389 & 41.822778 \\
\hline $8 / 28 / 0918: 40$ & $18: 43$ & 5703197 & Normal & OK & Normal $\left(26^{\circ} \mathrm{C}\right)$ & Normal $(54 \%)$ & OK & OK & 140 & -89.231111 & 41.850833 \\
\hline $8 / 28 / 09$ 18:45 & $18: 48$ & 5703197 & Normal & OK & Normal $\left(27^{\circ} \mathrm{C}\right)$ & Normal (52\%) & OK & OK & 140 & -89.125000 & 41.883333 \\
\hline $8 / 28 / 09$ 18:50 & $18: 54$ & 5703197 & Normal & OK & Normal $\left(27^{\circ} \mathrm{C}\right)$ & Normal (52\%) & OK & OK & 140 & -89.025000 & 41.904167 \\
\hline $8 / 28 / 09$ 18:55 & 19:00 & 5703197 & Normal & OK & Normal $\left(27^{\circ} \mathrm{C}\right)$ & Normal (52\%) & OK & OK & 140 & -88.913333 & 41.900000 \\
\hline $8 / 28 / 09$ 19:00 & 19:05 & 5703197 & Normal & OK & Normal $\left(27^{\circ} \mathrm{C}\right)$ & Normal $(52 \%)$ & OK & OK & 140 & -88.814444 & 41.900000 \\
\hline $8 / 28 / 09$ 19:05 & 19:11 & 5703197 & Normal & OK & Normal $\left(27^{\circ} \mathrm{C}\right)$ & Normal $(52 \%)$ & OK & OK & 140 & -88.739444 & 41.900000 \\
\hline $8 / 28 / 09$ 19:10 & $19: 16$ & 5703197 & Normal & OK & $\operatorname{Normal}\left(27^{\circ} \mathrm{C}\right)$ & Normal $(51 \%)$ & OK & OK & 140 & -88.739444 & 41.900000 \\
\hline $8 / 28 / 09$ 19:15 & $19: 22$ & 5703197 & Normal & OK & $\operatorname{Normal}\left(27^{\circ} \mathrm{C}\right)$ & Normal (51\%) & OK & OK & 140 & -88.737500 & 41.900000 \\
\hline $8 / 28 / 09$ 19:20 & $19: 27$ & 5703197 & Normal & OK & Normal $\left(27^{\circ} \mathrm{C}\right)$ & Normal $(51 \%)$ & OK & OK & 140 & -88.737500 & 41.900000 \\
\hline $8 / 28 / 09$ 19:25 & $19: 33$ & 5703197 & Normal & OK & Normal $\left(27^{\circ} \mathrm{C}\right)$ & Normal $(51 \%)$ & OK & OK & 140 & -88.639444 & 41.894722 \\
\hline $8 / 28 / 09$ 19:30 & $19: 38$ & 5703197 & Normal & OK & Normal $\left(27^{\circ} \mathrm{C}\right)$ & Normal (51\%) & OK & OK & 140 & -88.538333 & 41.859167 \\
\hline 8/28/09 19:35 & $19: 38$ & 5703197 & Normal & OK & Normal $\left(27^{\circ} \mathrm{C}\right)$ & Normal (51\%) & OK & OK & 140 & -88.448889 & 41.812500 \\
\hline $8 / 28 / 09$ 19:40 & $19: 44$ & 5703197 & Normal & OK & $\operatorname{Normal}\left(27^{\circ} \mathrm{C}\right)$ & Normal (51\%) & OK & OK & 140 & -88.347778 & 41.794722 \\
\hline $8 / 28 / 09$ 19:45 & $19: 49$ & 5703197 & Normal & OK & Normal $\left(27^{\circ} \mathrm{C}\right)$ & Normal $(51 \%)$ & OK & OK & 140 & -88.261389 & 41.800000 \\
\hline $8 / 28 / 09$ 19:50 & 19:55 & 5703197 & Normal & OK & Normal $\left(27^{\circ} \mathrm{C}\right)$ & Normal (51\%) & oK & OK & 140 & -88.156111 & 41.806111 \\
\hline $8 / 28 / 09$ 19:55 & $20: 00$ & 5703197 & Normal & OK & Normal $\left(27^{\circ} \mathrm{C}\right)$ & Normal (51\%) & OK & OK & 140 & -88.062500 & 41.810278 \\
\hline $8 / 28 / 0920: 00$ & 20:06 & 5703197 & Normal & OK & Normal $\left(27^{\circ} \mathrm{C}\right)$ & Normal (51\%) & OK & OK & 140 & -88.054167 & 41.807222 \\
\hline 8/28/09 20:05 & $20: 11$ & 5703197 & Normal & OK & $\operatorname{Normal}\left(27^{\circ} \mathrm{C}\right)$ & Normal $(52 \%)$ & OK & OK & 140 & -88.054167 & 41.781111 \\
\hline 8/28/09 20:10 & $20: 22$ & 5703197 & Normal & OK & $\operatorname{Normal}\left(27^{\circ} \mathrm{C}\right)$ & Normal $(52 \%)$ & OK & OK & 140 & -88.035278 & 41.732222 \\
\hline $8 / 28 / 0913: 40$ & $13: 44$ & 5714140 & Normal & OK & $\operatorname{Normal}\left(23^{\circ} \mathrm{C}\right)$ & Normal $(56 \%)$ & $\overline{O K}$ & $\overline{\mathrm{OK}}$ & 132 & -87.971667 & 41.732222 \\
\hline $8 / 28 / 0913: 45$ & $13: 49$ & 5714140 & Normal & OK & Normal $\left(23^{\circ} \mathrm{C}\right)$ & Normal $(56 \%)$ & OK & OK & 132 & -87.971667 & 41.732222 \\
\hline $8 / 28 / 0913: 50$ & $14: 00$ & 5714140 & Normal & OK & $\operatorname{Normal}\left(23^{\circ} \mathrm{C}\right)$ & Normal (56\%) & OK & OK & 132 & -87.971667 & 41.732222 \\
\hline $8 / 28 / 09$ 13:55 & $14: 00$ & 5714140 & Normal & OK & $\operatorname{Normal}\left(23^{\circ} \mathrm{C}\right)$ & Normal $(56 \%)$ & OK & OK & 132 & -87.971667 & 41.732222 \\
\hline $8 / 28 / 09$ 14:00 & $14: 06$ & 5714140 & Normal & OK & $\operatorname{Normal}\left(23^{\circ} \mathrm{C}\right)$ & Normal $(56 \%)$ & OK & OK & 132 & -88.068611 & 41.684167 \\
\hline $8 / 28 / 09$ 14:05 & $14: 11$ & 5714140 & Normal & OK & Normal $\left(23^{\circ} \mathrm{C}\right)$ & Normal $(56 \%)$ & OK & OK & 132 & -88.154167 & 41.640556 \\
\hline $8 / 28 / 0914: 10$ & $14: 17$ & 5714140 & Normal & OK & Normal $\left(23^{\circ} \mathrm{C}\right)$ & Normal $(56 \%)$ & OK & OK & 132 & -88.181111 & 41.568611 \\
\hline $8 / 28 / 0914: 15$ & $14: 22$ & 5714140 & Normal & OK & $\operatorname{Normal}\left(23^{\circ} \mathrm{C}\right)$ & Normal $(56 \%)$ & OK & OK & 132 & -88.197778 & 41.493611 \\
\hline $8 / 28 / 0914: 20$ & $14: 22$ & 5714140 & Normal & OK & $\operatorname{Normal}\left(23^{\circ} \mathrm{C}\right)$ & Normal $(56 \%)$ & OK & OK & 132 & -88.280000 & 41.462500 \\
\hline $8 / 28 / 09$ 14:25 & $14: 28$ & 5714140 & Normal & OK & Normal $\left(23^{\circ} \mathrm{C}\right)$ & Normal $(56 \%)$ & OK & OK & 132 & -88.371667 & 41.417500 \\
\hline $8 / 28 / 0914: 32$ & $14: 39$ & 5714140 & Normal & OK & Normal $\left(23^{\circ} \mathrm{C}\right)$ & Normal $(56 \%)$ & OK & OK & 132 & -88.422778 & 41.392500 \\
\hline $8 / 28 / 0914: 35$ & $14: 39$ & 5714140 & Normal & OK & $\operatorname{Normal}\left(23^{\circ} \mathrm{C}\right)$ & Normal $(56 \%)$ & OK & OK & 132 & -88.422778 & 41.392500 \\
\hline $8 / 28 / 0914: 40$ & $14: 44$ & 5714140 & Normal & OK & Normal $\left(23^{\circ} \mathrm{C}\right)$ & Normal (56\%) & OK & OK & 132 & -88.422778 & 41.392500 \\
\hline $8 / 28 / 0914: 45$ & $14: 50$ & 5714140 & Normal & OK & Normal $\left(23^{\circ} \mathrm{C}\right)$ & Normal (56\%) & OK & OK & 132 & -88.461389 & 41.378056 \\
\hline $8 / 28 / 0914: 50$ & $14: 56$ & 5714140 & Normal & OK & $\operatorname{Normal}\left(23^{\circ} \mathrm{C}\right)$ & Normal $(56 \%)$ & OK & OK & 132 & -88.575000 & 41.376944 \\
\hline $8 / 28 / 09$ 14:55 & $15: 01$ & 5714140 & Normal & OK & Normal $\left(23^{\circ} \mathrm{C}\right)$ & Normal (55\%) & OK & OK & 132 & -88.717500 & 41.375833 \\
\hline $8 / 28 / 09$ 15:00 & 15:07 & 5714140 & Normal & OK & $\operatorname{Normal}\left(23^{\circ} \mathrm{C}\right)$ & Normal (55\%) & OK & OK & 132 & -88.792500 & 41.376944 \\
\hline $8 / 28 / 09$ 15:05 & $15: 12$ & 5714140 & Normal & OK & $\operatorname{Normal}\left(23^{\circ} \mathrm{C}\right)$ & Normal (55\%) & OK & OK & 132 & -88.901944 & 41.368611 \\
\hline $8 / 28 / 0915: 10$ & $15: 12$ & 5714140 & Normal & OK & $\operatorname{Normal}\left(23^{\circ} \mathrm{C}\right)$ & Normal $(55 \%)$ & OK & OK & 132 & -89.014444 & 41.367500 \\
\hline $8 / 28 / 0915: 15$ & $15: 18$ & 5714140 & Normal & OK & Normal $\left(23^{\circ} \mathrm{C}\right)$ & Normal $(55 \%)$ & OK & OK & 132 & -89.058333 & 41.413333 \\
\hline $8 / 28 / 0915: 20$ & $15: 23$ & 5714140 & Normal & OK & Normal $\left(23^{\circ} \mathrm{C}\right)$ & Normal $(55 \%)$ & OK & OK & 132 & -89.050833 & 41.460278 \\
\hline $8 / 28 / 0915: 25$ & $15: 29$ & 5714140 & Normal & OK & $\operatorname{Normal}\left(23^{\circ} \mathrm{C}\right)$ & Normal $(55 \%)$ & OK & OK & 132 & -89.058333 & 41.375833 \\
\hline $8 / 28 / 0915: 30$ & $15: 35$ & 5714140 & Normal & OK & Normal $\left(23^{\circ} \mathrm{C}\right)$ & Normal $(55 \%)$ & OK & OK & 132 & -89.125833 & 41.368611 \\
\hline
\end{tabular}




\begin{tabular}{|c|c|c|c|c|c|c|c|c|c|c|c|}
\hline Event Time & at QC Hub & Tag ID & Tag Status & Seal & Temperature & Humidity & Shock & Battery & RSSI & Longitude & Latitude \\
\hline $8 / 28 / 09$ 15:35 & $15: 40$ & 5714140 & Normal & OK & Normal $\left(23^{\circ} \mathrm{C}\right)$ & Normal (55\%) & OK & OK & 132 & -89.125833 & 41.368611 \\
\hline 8/28/09 15:40 & $15: 46$ & 5714140 & Normal & OK & Normal $\left(23^{\circ} \mathrm{C}\right)$ & Normal (55\%) & OK & OK & 132 & -89.125833 & 41.368611 \\
\hline $8 / 28 / 09$ 15:45 & $15: 51$ & 5714140 & Normal & OK & Normal $\left(23^{\circ} \mathrm{C}\right)$ & Normal (55\%) & OK & OK & 132 & -89.200000 & 41.364444 \\
\hline $8 / 28 / 09$ 15:50 & $15: 57$ & 5714140 & Normal & OK & Normal $\left(23^{\circ} \mathrm{C}\right)$ & Normal (55\%) & OK & OK & 132 & -89.240556 & 41.371667 \\
\hline $8 / 28 / 0916: 00$ & $16: 03$ & 5714140 & Normal & OK & Normal $\left(23^{\circ} \mathrm{C}\right)$ & Normal (55\%) & OK & OK & 132 & -89.458333 & 41.400833 \\
\hline $8 / 28 / 09$ 16:05 & $16: 13$ & 5714140 & Normal & OK & Normal $\left(23^{\circ} \mathrm{C}\right)$ & Normal (55\%) & OK & OK & 132 & -89.558333 & 41.393611 \\
\hline 8/28/09 16:11 & $16: 14$ & 5714140 & Normal & OK & Normal $\left(23^{\circ} \mathrm{C}\right)$ & Normal (55\%) & OK & OK & 132 & -89.558333 & 41.393611 \\
\hline 8/28/09 16:15 & $16: 19$ & 5714140 & Normal & OK & Normal $\left(23^{\circ} \mathrm{C}\right)$ & Normal (55\%) & OK & OK & 132 & -89.558333 & 41.393611 \\
\hline $8 / 28 / 09$ 16:20 & $16: 25$ & 5714140 & Normal & OK & Normal $\left(23^{\circ} \mathrm{C}\right)$ & Normal (55\%) & OK & OK & 132 & -89.558333 & 41.393611 \\
\hline $8 / 28 / 0916: 25$ & $16: 30$ & 5714140 & Normal & OK & Normal $\left(23^{\circ} \mathrm{C}\right)$ & Normal (55\%) & OK & OK & 132 & -89.623889 & 41.394722 \\
\hline $8 / 28 / 09$ 16:30 & $16: 36$ & 5714140 & Normal & OK & Normal $\left(23^{\circ} \mathrm{C}\right)$ & Normal $(55 \%)$ & OK & OK & 132 & -89.725833 & 41.387500 \\
\hline $8 / 28 / 0916: 35$ & $16: 41$ & 5714140 & Normal & OK & Normal $\left(23^{\circ} \mathrm{C}\right)$ & Normal (55\%) & OK & $\mathrm{OK}$ & 132 & -89.834167 & 41.388333 \\
\hline $8 / 28 / 0916: 40$ & $16: 47$ & 5714140 & Normal & OK & Normal $\left(23^{\circ} \mathrm{C}\right)$ & Normal (55\%) & OK & $\mathrm{OK}$ & 132 & -89.941667 & 41.416667 \\
\hline $8 / 28 / 0916: 45$ & $16: 52$ & 5714140 & Normal & OK & Normal $\left(23^{\circ} \mathrm{C}\right)$ & Normal (55\%) & OK & OK & 132 & -90.050000 & 41.413333 \\
\hline $8 / 28 / 0916: 50$ & $16: 52$ & 5714140 & Normal & $\mathrm{OK}$ & Normal $\left(23^{\circ} \mathrm{C}\right)$ & Normal (55\%) & OK & OK & 132 & -90.157222 & 41.430000 \\
\hline 8/28/09 16:55 & $16: 58$ & 5714140 & Normal & $\mathrm{OK}$ & Normal $\left(23^{\circ} \mathrm{C}\right)$ & Normal (55\%) & OK & OK & 132 & -90.267500 & 41.440556 \\
\hline 8/28/09 17:00 & $17: 09$ & 5714140 & Normal & OK & Normal $\left(23^{\circ} \mathrm{C}\right)$ & Normal (55\%) & OK & OK & 132 & -90.338333 & 41.476944 \\
\hline 8/28/09 17:05 & $17: 09$ & 5714140 & Normal & $\mathrm{OK}$ & Normal $\left(23^{\circ} \mathrm{C}\right)$ & Normal (55\%) & $\mathrm{OK}$ & OK & 132 & -90.334167 & 41.486389 \\
\hline 8/28/09 17:11 & $17: 15$ & 5714140 & Normal & OK & Normal $\left(23^{\circ} \mathrm{C}\right)$ & Normal (55\%) & OK & OK & 132 & -90.334167 & 41.486389 \\
\hline 8/28/09 17:15 & $17: 20$ & 5714140 & Normal & OK & Normal $\left(23^{\circ} \mathrm{C}\right)$ & Normal (55\%) & OK & OK & 132 & -90.334167 & 41.486389 \\
\hline 8/28/09 17:20 & $17: 26$ & 5714140 & Normal & OK & Normal $\left(23^{\circ} \mathrm{C}\right)$ & Normal (55\%) & OK & OK & 132 & -90.334167 & 41.486389 \\
\hline 8/28/09 17:25 & $17: 31$ & 5714140 & Normal & OK & Normal $\left(23^{\circ} \mathrm{C}\right)$ & Normal (55\%) & $\mathrm{OK}$ & OK & 132 & -90.340556 & 41.511389 \\
\hline 8/28/09 17:30 & $17: 37$ & 5714140 & Normal & OK & Normal $\left(23^{\circ} \mathrm{C}\right)$ & Normal (55\%) & OK & OK & 132 & -90.234167 & 41.562500 \\
\hline 8/28/09 17:35 & $17: 42$ & 5714140 & Normal & OK & Normal $\left(23^{\circ} \mathrm{C}\right)$ & Normal (55\%) & OK & OK & 132 & -90.209167 & 41.581111 \\
\hline 8/28/09 17:40 & $17: 42$ & 5714140 & Normal & OK & Normal $\left(23^{\circ} \mathrm{C}\right)$ & Normal (55\%) & OK & OK & 132 & -90.163333 & 41.642500 \\
\hline 8/28/09 17:45 & $17: 48$ & 5714140 & Normal & OK & Normal $\left(23^{\circ} \mathrm{C}\right)$ & Normal (55\%) & OK & OK & 132 & -90.080000 & 41.690556 \\
\hline $8 / 28 / 09$ 17:50 & $17: 53$ & 5714140 & Normal & OK & Normal $\left(23^{\circ} \mathrm{C}\right)$ & Normal (55\%) & OK & OK & 132 & -89.983333 & 41.718611 \\
\hline $8 / 28 / 09$ 17:55 & $17: 59$ & 5714140 & Normal & OK & Normal $\left(25^{\circ} \mathrm{C}\right)$ & Normal (54\%) & OK & OK & 132 & -89.876944 & 41.744722 \\
\hline $8 / 28 / 09$ 18:00 & $18: 04$ & 5714140 & Normal & OK & Normal $\left(25^{\circ} \mathrm{C}\right)$ & Normal (54\%) & OK & OK & 132 & -89.775000 & 41.757222 \\
\hline $8 / 28 / 09$ 18:05 & $18: 10$ & 5714140 & Normal & OK & Normal $\left(25^{\circ} \mathrm{C}\right)$ & Normal (54\%) & OK & OK & 132 & -89.689444 & 41.750833 \\
\hline 8/28/09 18:10 & $18: 15$ & 5714140 & Normal & OK & Normal $\left(25^{\circ} \mathrm{C}\right)$ & Normal (54\%) & OK & OK & 132 & -89.689444 & 41.762500 \\
\hline 8/28/09 18:15 & $18: 21$ & 5714140 & Normal & OK & Normal $\left(25^{\circ} \mathrm{C}\right)$ & Normal (54\%) & OK & OK & 132 & -89.678056 & 41.750833 \\
\hline $8 / 28 / 09$ 18:20 & $18: 26$ & 5714140 & Normal & OK & Normal $\left(25^{\circ} \mathrm{C}\right)$ & Normal (54\%) & OK & OK & 132 & -89.635278 & 41.759167 \\
\hline $8 / 28 / 09$ 18:25 & $18: 32$ & 5714140 & Normal & OK & Normal $\left(25^{\circ} \mathrm{C}\right)$ & Normal (54\%) & OK & OK & 132 & -89.532222 & 41.789444 \\
\hline $8 / 28 / 0918: 30$ & $18: 38$ & 5714140 & Normal & OK & Normal $\left(25^{\circ} \mathrm{C}\right)$ & Normal (54\%) & OK & OK & 132 & -89.432222 & 41.822778 \\
\hline $8 / 28 / 0918: 35$ & $18: 38$ & 5714140 & Normal & OK & Normal $\left(25^{\circ} \mathrm{C}\right)$ & Normal (54\%) & OK & OK & 132 & -89.336389 & 41.822778 \\
\hline $8 / 28 / 09$ 18:40 & $18: 43$ & 5714140 & Normal & OK & Normal $\left(25^{\circ} \mathrm{C}\right)$ & Normal (54\%) & OK & OK & 132 & -89.231111 & 41.850833 \\
\hline $8 / 28 / 09$ 18:45 & $18: 48$ & 5714140 & Normal & OK & Normal $\left(25^{\circ} \mathrm{C}\right)$ & Normal (53\%) & OK & OK & 132 & -89.125000 & 41.883333 \\
\hline $8 / 28 / 09$ 18:50 & $18: 54$ & 5714140 & Normal & OK & Normal $\left(25^{\circ} \mathrm{C}\right)$ & Normal (53\%) & OK & OK & 132 & -89.025000 & 41.904167 \\
\hline $8 / 28 / 09$ 18:55 & $19: 00$ & 5714140 & Normal & OK & Normal $\left(25^{\circ} \mathrm{C}\right)$ & Normal (53\%) & OK & OK & 132 & -88.913333 & 41.900000 \\
\hline 8/28/09 19:00 & 19:05 & 5714140 & Normal & OK & Normal $\left(25^{\circ} \mathrm{C}\right)$ & Normal $(53 \%)$ & OK & OK & 132 & -88.814444 & 41.900000 \\
\hline 8/28/09 19:05 & $19: 11$ & 5714140 & Normal & OK & Normal $\left(25^{\circ} \mathrm{C}\right)$ & Normal (53\%) & OK & OK & 132 & -88.739444 & 41.900000 \\
\hline $8 / 28 / 09$ 19:10 & $19: 16$ & 5714140 & Normal & OK & Normal $\left(25^{\circ} \mathrm{C}\right)$ & Normal (53\%) & OK & OK & 132 & -88.739444 & 41.900000 \\
\hline $8 / 28 / 09$ 19:15 & $19: 22$ & 5714140 & Normal & OK & Normal $\left(25^{\circ} \mathrm{C}\right)$ & Normal (53\%) & OK & OK & 132 & -88.737500 & 41.900000 \\
\hline $8 / 28 / 09$ 19:20 & $19: 27$ & 5714140 & Normal & OK & Normal $\left(25^{\circ} \mathrm{C}\right)$ & Normal $(53 \%)$ & OK & OK & 132 & -88.737500 & 41.900000 \\
\hline $8 / 28 / 09$ 19:25 & $19: 33$ & 5714140 & Normal & OK & Normal $\left(25^{\circ} \mathrm{C}\right)$ & Normal (53\%) & OK & OK & 132 & -88.639444 & 41.894722 \\
\hline $8 / 28 / 09$ 19:30 & $19: 38$ & 5714140 & Normal & OK & Normal $\left(25^{\circ} \mathrm{C}\right)$ & Normal (53\%) & OK & OK & 132 & -88.538333 & 41.859167 \\
\hline $8 / 28 / 09$ 19:35 & $19: 38$ & 5714140 & Normal & OK & Normal $\left(25^{\circ} \mathrm{C}\right)$ & Normal (53\%) & OK & OK & 132 & -88.448889 & 41.812500 \\
\hline $8 / 28 / 0919: 40$ & $19: 44$ & 5714140 & Normal & OK & Normal $\left(25^{\circ} \mathrm{C}\right)$ & Normal (53\%) & OK & OK & 132 & -88.347778 & 41.794722 \\
\hline 8/28/09 19:45 & $19: 49$ & 5714140 & Normal & OK & Normal $\left(25^{\circ} \mathrm{C}\right)$ & Normal (53\%) & OK & OK & 132 & -88.261389 & 41.800000 \\
\hline $8 / 28 / 09$ 19:50 & 19:55 & 5714140 & Normal & OK & Normal $\left(25^{\circ} \mathrm{C}\right)$ & Normal (53\%) & $\mathrm{OK}$ & OK & 132 & -88.156111 & 41.806111 \\
\hline 8/28/09 19:55 & $20: 00$ & 5714140 & Normal & OK & Normal $\left(25^{\circ} \mathrm{C}\right)$ & Normal (53\%) & OK & OK & 132 & -88.062500 & 41.810278 \\
\hline $8 / 28 / 09$ 20:00 & $20: 06$ & 5714140 & Normal & OK & Normal $\left(25^{\circ} \mathrm{C}\right)$ & Normal (53\%) & OK & OK & 132 & -88.054167 & 41.807222 \\
\hline 8/28/09 20:05 & 20:11 & 5714140 & Normal & OK & Normal $\left(25^{\circ} \mathrm{C}\right)$ & Normal (54\%) & OK & OK & 132 & -88.054167 & 41.781111 \\
\hline $8 / 28 / 09$ 20:10 & $20: 22$ & 5714140 & Normal & $\mathrm{OK}$ & Normal $\left(25^{\circ} \mathrm{C}\right)$ & Normal (54\%) & $\mathrm{OK}$ & $\mathrm{OK}$ & 132 & -88.035278 & 41.732222 \\
\hline $8 / 28 / 0913: 40$ & $13: 44$ & 5714145 & Normal & OK & Normal $\left(23^{\circ} \mathrm{C}\right)$ & Normal (56\%) & $\overline{O K}$ & $\overline{O K}$ & 138 & -87.971667 & 41.732222 \\
\hline 8/28/09 13:45 & $13: 49$ & 5714145 & Normal & OK & Normal $\left(23^{\circ} \mathrm{C}\right)$ & Normal (56\%) & OK & OK & 138 & -87.971667 & 41.732222 \\
\hline $8 / 28 / 09$ 13:50 & $14: 00$ & 5714145 & Normal & OK & Normal $\left(23^{\circ} \mathrm{C}\right)$ & Normal (56\%) & OK & OK & 138 & -87.971667 & 41.732222 \\
\hline $8 / 28 / 09$ 13:55 & $14: 00$ & 5714145 & Normal & OK & Normal $\left(23^{\circ} \mathrm{C}\right)$ & Normal (56\%) & OK & OK & 138 & -87.971667 & 41.732222 \\
\hline $8 / 28 / 09$ 14:00 & $14: 06$ & 5714145 & Normal & OK & Normal $\left(23^{\circ} \mathrm{C}\right)$ & Normal (56\%) & OK & OK & 138 & -88.068611 & 41.684167 \\
\hline $8 / 28 / 09$ 14:05 & $14: 11$ & 5714145 & Normal & OK & Normal $\left(23^{\circ} \mathrm{C}\right)$ & Normal (56\%) & OK & OK & 138 & -88.154167 & 41.640556 \\
\hline $8 / 28 / 0914: 10$ & $14: 17$ & 5714145 & Normal & OK & Normal $\left(23^{\circ} \mathrm{C}\right)$ & Normal (56\%) & OK & OK & 138 & -88.181111 & 41.568611 \\
\hline $8 / 28 / 0914: 15$ & $14: 22$ & 5714145 & Normal & OK & Normal $\left(23^{\circ} \mathrm{C}\right)$ & Normal (56\%) & OK & OK & 138 & -88.197778 & 41.493611 \\
\hline $8 / 28 / 0914: 20$ & $14: 22$ & 5714145 & Normal & OK & Normal $\left(23^{\circ} \mathrm{C}\right)$ & Normal $(56 \%)$ & OK & OK & 138 & -88.280000 & 41.462500 \\
\hline $8 / 28 / 0914: 25$ & $14: 28$ & 5714145 & Normal & OK & Normal $\left(23^{\circ} \mathrm{C}\right)$ & Normal (56\%) & OK & $\mathrm{OK}$ & 138 & -88.371667 & 41.417500 \\
\hline $8 / 28 / 09$ 14:32 & $14: 39$ & 5714145 & Normal & OK & Normal $\left(23^{\circ} \mathrm{C}\right)$ & Normal (56\%) & OK & OK & 138 & -88.422778 & 41.392500 \\
\hline $8 / 28 / 09$ 14:35 & $14: 39$ & 5714145 & Normal & OK & Normal $\left(23^{\circ} \mathrm{C}\right)$ & Normal (56\%) & OK & OK & 138 & -88.422778 & 41.392500 \\
\hline $8 / 28 / 09$ 14:40 & $14: 44$ & 5714145 & Normal & OK & Normal $\left(23^{\circ} \mathrm{C}\right)$ & Normal (56\%) & OK & OK & 138 & -88.422778 & 41.392500 \\
\hline $8 / 28 / 09$ 14:45 & $14: 50$ & 5714145 & Normal & OK & Normal $\left(23^{\circ} \mathrm{C}\right)$ & Normal (56\%) & OK & OK & 138 & -88.461389 & 41.378056 \\
\hline $8 / 28 / 0914: 50$ & $14: 56$ & 5714145 & Normal & OK & Normal $\left(23^{\circ} \mathrm{C}\right)$ & Normal (56\%) & OK & OK & 138 & -88.575000 & 41.376944 \\
\hline $8 / 28 / 09$ 14:55 & $15: 01$ & 5714145 & Normal & OK & Normal $\left(23^{\circ} \mathrm{C}\right)$ & Normal (56\%) & OK & OK & 138 & -88.717500 & 41.375833 \\
\hline $8 / 28 / 09$ 15:00 & $15: 07$ & 5714145 & Normal & OK & Normal $\left(23^{\circ} \mathrm{C}\right)$ & Normal (56\%) & OK & OK & 138 & -88.792500 & 41.376944 \\
\hline 8/28/09 15:05 & $15: 12$ & 5714145 & Normal & OK & Normal $\left(23^{\circ} \mathrm{C}\right)$ & Normal (56\%) & OK & OK & 138 & -88.901944 & 41.368611 \\
\hline $8 / 28 / 09$ 15:10 & $15: 12$ & 5714145 & Normal & OK & Normal $\left(23^{\circ} \mathrm{C}\right)$ & Normal (56\%) & OK & OK & 138 & -89.014444 & 41.367500 \\
\hline $8 / 28 / 09$ 15:15 & $15: 18$ & 5714145 & Normal & OK & Normal $\left(23^{\circ} \mathrm{C}\right)$ & Normal (56\%) & OK & OK & 138 & -89.058333 & 41.413333 \\
\hline $8 / 28 / 09$ 15:20 & $15: 23$ & 5714145 & Normal & OK & Normal $\left(23^{\circ} \mathrm{C}\right)$ & Normal (56\%) & OK & OK & 138 & -89.050833 & 41.460278 \\
\hline $8 / 28 / 09$ 15:25 & $15: 29$ & 5714145 & Normal & OK & Normal $\left(23^{\circ} \mathrm{C}\right)$ & Normal (56\%) & OK & OK & 138 & -89.058333 & 41.375833 \\
\hline $8 / 28 / 09$ 15:30 & $15: 35$ & 5714145 & Normal & OK & Normal $\left(23^{\circ} \mathrm{C}\right)$ & Normal (56\%) & OK & OK & 138 & -89.125833 & 41.368611 \\
\hline $8 / 28 / 09$ 15:35 & $15: 40$ & 5714145 & Normal & OK & Normal $\left(23^{\circ} \mathrm{C}\right)$ & Normal $(56 \%)$ & OK & OK & 138 & -89.125833 & 41.368611 \\
\hline $8 / 28 / 09$ 15:40 & $15: 46$ & 5714145 & Normal & OK & Normal $\left(23^{\circ} \mathrm{C}\right)$ & Normal (56\%) & OK & OK & 138 & -89.125833 & 41.368611 \\
\hline 8/28/09 15:45 & $15: 51$ & 5714145 & Normal & OK & Normal $\left(23^{\circ} \mathrm{C}\right)$ & Normal (56\%) & OK & OK & 138 & -89.200000 & 41.364444 \\
\hline
\end{tabular}




\begin{tabular}{|c|c|c|c|c|c|c|c|c|c|c|c|}
\hline Event Time & at QC Hub & Tag ID & Tag Status & Seal & Temperature & Humidity & Shock & Battery & RSSI & Longitude & Latitude \\
\hline $8 / 28 / 09$ 15:50 & $15: 57$ & 5714145 & Normal & OK & Normal $\left(23^{\circ} \mathrm{C}\right)$ & Normal (56\%) & OK & OK & 138 & -89.240556 & 41.371667 \\
\hline 8/28/09 16:00 & $16: 03$ & 5714145 & Normal & OK & Normal $\left(23^{\circ} \mathrm{C}\right)$ & Normal (56\%) & OK & OK & 138 & -89.458333 & 41.400833 \\
\hline 8/28/09 16:05 & $16: 13$ & 5714145 & Normal & OK & Normal $\left(23^{\circ} \mathrm{C}\right)$ & Normal (56\%) & OK & OK & 138 & -89.558333 & 41.393611 \\
\hline $8 / 28 / 09$ 16:11 & $16: 14$ & 5714145 & Normal & OK & Normal $\left(23^{\circ} \mathrm{C}\right)$ & Normal (56\%) & OK & OK & 138 & -89.558333 & 41.393611 \\
\hline 8/28/09 16:15 & $16: 19$ & 5714145 & Normal & OK & Normal $\left(23^{\circ} \mathrm{C}\right)$ & Normal (56\%) & OK & OK & 138 & -89.558333 & 41.393611 \\
\hline $8 / 28 / 09$ 16:20 & $16: 25$ & 5714145 & Normal & OK & Normal $\left(23^{\circ} \mathrm{C}\right)$ & Normal (56\%) & OK & OK & 138 & -89.558333 & 41.393611 \\
\hline $8 / 28 / 09$ 16:25 & $16: 30$ & 5714145 & Normal & OK & Normal $\left(23^{\circ} \mathrm{C}\right)$ & Normal (56\%) & OK & OK & 138 & -89.623889 & 41.394722 \\
\hline $8 / 28 / 0916: 30$ & $16: 36$ & 5714145 & Normal & OK & Normal $\left(23^{\circ} \mathrm{C}\right)$ & Normal (56\%) & OK & OK & 138 & -89.725833 & 41.387500 \\
\hline 8/28/09 16:35 & $16: 41$ & 5714145 & Normal & OK & Normal $\left(23^{\circ} \mathrm{C}\right)$ & Normal (56\%) & OK & OK & 138 & -89.834167 & 41.388333 \\
\hline $8 / 28 / 0916: 40$ & $16: 47$ & 5714145 & Normal & OK & Normal $\left(23^{\circ} \mathrm{C}\right)$ & Normal (56\%) & OK & OK & 138 & -89.941667 & 41.416667 \\
\hline $8 / 28 / 09$ 16:45 & $16: 52$ & 5714145 & Normal & OK & Normal $\left(23^{\circ} \mathrm{C}\right)$ & Normal (56\%) & OK & OK & 138 & -90.050000 & 41.413333 \\
\hline $8 / 28 / 09$ 16:50 & $16: 52$ & 5714145 & Normal & OK & Normal $\left(23^{\circ} \mathrm{C}\right)$ & Normal (56\%) & OK & OK & 138 & -90.157222 & 41.430000 \\
\hline $8 / 28 / 09$ 16:55 & $16: 58$ & 5714145 & Normal & OK & Normal $\left(23^{\circ} \mathrm{C}\right)$ & Normal (56\%) & OK & OK & 138 & -90.267500 & 41.440556 \\
\hline 8/28/09 17:00 & $17: 09$ & 5714145 & Normal & OK & Normal $\left(23^{\circ} \mathrm{C}\right)$ & Normal $(56 \%)$ & OK & OK & 138 & -90.338333 & 41.476944 \\
\hline $8 / 28 / 09$ 17:05 & $17: 09$ & 5714145 & Normal & OK & Normal $\left(23^{\circ} \mathrm{C}\right)$ & Normal $(56 \%)$ & OK & OK & 138 & -90.334167 & 41.486389 \\
\hline $8 / 28 / 09$ 17:11 & $17: 15$ & 5714145 & Normal & OK & Normal $\left(23^{\circ} \mathrm{C}\right)$ & Normal (56\%) & OK & OK & 138 & -90.334167 & 41.486389 \\
\hline $8 / 28 / 09$ 17:15 & $17: 20$ & 5714145 & Normal & OK & Normal $\left(23^{\circ} \mathrm{C}\right)$ & Normal (56\%) & OK & OK & 138 & -90.334167 & 41.486389 \\
\hline $8 / 28 / 09$ 17:20 & $17: 26$ & 5714145 & Normal & OK & Normal $\left(23^{\circ} \mathrm{C}\right)$ & Normal $(56 \%)$ & OK & OK & 138 & -90.334167 & 41.486389 \\
\hline $8 / 28 / 09$ 17:25 & $17: 31$ & 5714145 & Normal & OK & Normal $\left(23^{\circ} \mathrm{C}\right)$ & Normal $(56 \%)$ & OK & OK & 138 & -90.340556 & 41.511389 \\
\hline $8 / 28 / 09$ 17:30 & $17: 37$ & 5714145 & Normal & OK & Normal $\left(23^{\circ} \mathrm{C}\right)$ & Normal (56\%) & OK & $\mathrm{OK}$ & 138 & -90.234167 & 41.562500 \\
\hline $8 / 28 / 09$ 17:35 & $17: 42$ & 5714145 & Normal & OK & Normal $\left(23^{\circ} \mathrm{C}\right)$ & Normal (56\%) & OK & OK & 138 & -90.209167 & 41.581111 \\
\hline $8 / 28 / 09$ 17:40 & $17: 42$ & 5714145 & Normal & OK & Normal $\left(23^{\circ} \mathrm{C}\right)$ & Normal $(56 \%)$ & OK & OK & 138 & -90.163333 & 41.642500 \\
\hline $8 / 28 / 09$ 17:45 & $17: 48$ & 5714145 & Normal & OK & Normal $\left(23^{\circ} \mathrm{C}\right)$ & Normal (56\%) & OK & OK & 138 & -90.080000 & 41.690556 \\
\hline $8 / 28 / 09$ 17:50 & $17: 53$ & 5714145 & Normal & OK & Normal $\left(23^{\circ} \mathrm{C}\right)$ & Normal (56\%) & OK & OK & 138 & -89.983333 & 41.718611 \\
\hline $8 / 28 / 09$ 17:55 & $17: 59$ & 5714145 & Normal & OK & Normal $\left(25^{\circ} \mathrm{C}\right)$ & Normal (55\%) & OK & OK & 138 & -89.876944 & 41.744722 \\
\hline $8 / 28 / 09$ 18:00 & $18: 04$ & 5714145 & Normal & OK & Normal $\left(25^{\circ} \mathrm{C}\right)$ & Normal $(55 \%)$ & OK & OK & 138 & -89.775000 & 41.757222 \\
\hline $8 / 28 / 09$ 18:05 & $18: 10$ & 5714145 & Normal & OK & Normal $\left(25^{\circ} \mathrm{C}\right)$ & Normal $(55 \%)$ & OK & OK & 138 & -89.689444 & 41.750833 \\
\hline $8 / 28 / 0918: 10$ & $18: 15$ & 5714145 & Normal & OK & Normal $\left(25^{\circ} \mathrm{C}\right)$ & Normal (55\%) & OK & OK & 138 & -89.689444 & 41.762500 \\
\hline $8 / 28 / 09$ 18:15 & $18: 21$ & 5714145 & Normal & OK & Normal $\left(25^{\circ} \mathrm{C}\right)$ & Normal (55\%) & OK & OK & 138 & -89.678056 & 41.750833 \\
\hline $8 / 28 / 0918: 20$ & $18: 26$ & 5714145 & Normal & OK & Normal $\left(25^{\circ} \mathrm{C}\right)$ & Normal (55\%) & OK & OK & 138 & -89.635278 & 41.759167 \\
\hline $8 / 28 / 09$ 18:25 & $18: 32$ & 5714145 & Normal & OK & Normal $\left(25^{\circ} \mathrm{C}\right)$ & Normal (55\%) & OK & OK & 138 & -89.532222 & 41.789444 \\
\hline $8 / 28 / 09$ 18:30 & $18: 38$ & 5714145 & Normal & OK & Normal $\left(25^{\circ} \mathrm{C}\right)$ & Normal (55\%) & OK & OK & 138 & -89.432222 & 41.822778 \\
\hline $8 / 28 / 09$ 18:35 & $18: 38$ & 5714145 & Normal & OK & Normal $\left(25^{\circ} \mathrm{C}\right)$ & Normal (55\%) & OK & OK & 138 & -89.336389 & 41.822778 \\
\hline $8 / 28 / 09$ 18:40 & $18: 43$ & 5714145 & Normal & OK & Normal $\left(25^{\circ} \mathrm{C}\right)$ & Normal (55\%) & OK & OK & 138 & -89.231111 & 41.850833 \\
\hline $8 / 28 / 09$ 18:45 & $18: 48$ & 5714145 & Normal & OK & Normal $\left(25^{\circ} \mathrm{C}\right)$ & Normal (54\%) & OK & OK & 138 & -89.125000 & 41.883333 \\
\hline $8 / 28 / 09$ 18:50 & $18: 54$ & 5714145 & Normal & OK & Normal $\left(25^{\circ} \mathrm{C}\right)$ & Normal (54\%) & OK & OK & 138 & -89.025000 & 41.904167 \\
\hline $8 / 28 / 09$ 18:55 & 19:00 & 5714145 & Normal & OK & Normal $\left(25^{\circ} \mathrm{C}\right)$ & Normal (54\%) & OK & OK & 138 & -88.913333 & 41.900000 \\
\hline $8 / 28 / 09$ 19:00 & $19: 05$ & 5714145 & Normal & OK & Normal $\left(25^{\circ} \mathrm{C}\right)$ & Normal (54\%) & OK & OK & 138 & -88.814444 & 41.900000 \\
\hline 8/28/09 19:05 & $19: 11$ & 5714145 & Normal & OK & Normal $\left(25^{\circ} \mathrm{C}\right)$ & Normal (54\%) & OK & OK & 138 & -88.739444 & 41.900000 \\
\hline $8 / 28 / 09$ 19:10 & $19: 16$ & 5714145 & Normal & OK & Normal $\left(25^{\circ} \mathrm{C}\right)$ & Normal (54\%) & OK & $\mathrm{OK}$ & 138 & -88.739444 & 41.900000 \\
\hline $8 / 28 / 09$ 19:15 & $19: 22$ & 5714145 & Normal & OK & Normal $\left(25^{\circ} \mathrm{C}\right)$ & Normal $(54 \%)$ & OK & OK & 138 & -88.737500 & 41.900000 \\
\hline $8 / 28 / 09$ 19:20 & $19: 27$ & 5714145 & Normal & OK & Normal $\left(25^{\circ} \mathrm{C}\right)$ & Normal (54\%) & OK & OK & 138 & -88.737500 & 41.900000 \\
\hline $8 / 28 / 09$ 19:25 & $19: 33$ & 5714145 & Normal & OK & Normal $\left(25^{\circ} \mathrm{C}\right)$ & Normal (54\%) & OK & OK & 138 & -88.639444 & 41.894722 \\
\hline $8 / 28 / 09$ 19:30 & $19: 38$ & 5714145 & Normal & OK & Normal $\left(25^{\circ} \mathrm{C}\right)$ & Normal (54\%) & OK & OK & 138 & -88.538333 & 41.859167 \\
\hline $8 / 28 / 09$ 19:35 & $19: 38$ & 5714145 & Normal & OK & Normal $\left(25^{\circ} \mathrm{C}\right)$ & Normal $(54 \%)$ & OK & OK & 138 & -88.448889 & 41.812500 \\
\hline $8 / 28 / 09$ 19:40 & $19: 44$ & 5714145 & Normal & OK & Normal $\left(25^{\circ} \mathrm{C}\right)$ & Normal $(54 \%)$ & OK & OK & 138 & -88.347778 & 41.794722 \\
\hline $8 / 28 / 09$ 19:45 & $19: 49$ & 5714145 & Normal & OK & Normal $\left(25^{\circ} \mathrm{C}\right)$ & Normal $(54 \%)$ & OK & OK & 138 & -88.261389 & 41.800000 \\
\hline $8 / 28 / 09$ 19:50 & $19: 55$ & 5714145 & Normal & OK & Normal $\left(25^{\circ} \mathrm{C}\right)$ & Normal (54\%) & OK & $\mathrm{OK}$ & 138 & -88.156111 & 41.806111 \\
\hline $8 / 28 / 09$ 19:55 & $20: 00$ & 5714145 & Normal & OK & Normal $\left(25^{\circ} \mathrm{C}\right)$ & Normal (54\%) & OK & OK & 138 & -88.062500 & 41.810278 \\
\hline $8 / 28 / 0920: 00$ & $20: 06$ & 5714145 & Normal & OK & Normal $\left(25^{\circ} \mathrm{C}\right)$ & Normal (54\%) & OK & OK & 138 & -88.054167 & 41.807222 \\
\hline $8 / 28 / 0920: 05$ & $20: 11$ & 5714145 & Normal & OK & Normal $\left(25^{\circ} \mathrm{C}\right)$ & Normal (54\%) & OK & OK & 138 & -88.054167 & 41.781111 \\
\hline $8 / 28 / 09$ 20:10 & $20: 22$ & 5714145 & Normal & $\mathrm{OK}$ & Normal $\left(25^{\circ} \mathrm{C}\right)$ & Normal (54\%) & OK & OK & 138 & -88.035278 & 41.732222 \\
\hline $8 / 28 / 0913: 40$ & $13: 44$ & 5714150 & Normal & OK & Normal $\left(23^{\circ} \mathrm{C}\right)$ & Normal (56\%) & $\mathrm{OK}$ & $\mathrm{OK}$ & 139 & -87.971667 & 41.732222 \\
\hline $8 / 28 / 09$ 13:45 & $13: 49$ & 5714150 & Normal & OK & Normal $\left(23^{\circ} \mathrm{C}\right)$ & Normal (56\%) & OK & OK & 139 & -87.971667 & 41.732222 \\
\hline $8 / 28 / 09$ 13:50 & $14: 00$ & 5714150 & Normal & OK & Normal $\left(23^{\circ} \mathrm{C}\right)$ & Normal (56\%) & OK & OK & 139 & -87.971667 & 41.732222 \\
\hline $8 / 28 / 0913: 55$ & $14: 00$ & 5714150 & Normal & OK & Normal $\left(23^{\circ} \mathrm{C}\right)$ & Normal (56\%) & OK & OK & 139 & -87.971667 & 41.732222 \\
\hline $8 / 28 / 0914: 00$ & $14: 06$ & 5714150 & Normal & OK & Normal $\left(23^{\circ} \mathrm{C}\right)$ & Normal (56\%) & OK & OK & 139 & -88.068611 & 41.684167 \\
\hline $8 / 28 / 09$ 14:05 & $14: 11$ & 5714150 & Normal & OK & Normal $\left(23^{\circ} \mathrm{C}\right)$ & Normal (56\%) & OK & OK & 139 & -88.154167 & 41.640556 \\
\hline $8 / 28 / 09$ 14:10 & $14: 17$ & 5714150 & Normal & OK & Normal $\left(23^{\circ} \mathrm{C}\right)$ & Normal (56\%) & OK & OK & 139 & -88.181111 & 41.568611 \\
\hline $8 / 28 / 09$ 14:15 & $14: 22$ & 5714150 & Normal & OK & Normal $\left(23^{\circ} \mathrm{C}\right)$ & Normal (56\%) & OK & OK & 139 & -88.197778 & 41.493611 \\
\hline $8 / 28 / 09$ 14:20 & $14: 22$ & 5714150 & Normal & OK & Normal $\left(23^{\circ} \mathrm{C}\right)$ & Normal (56\%) & OK & OK & 139 & -88.280000 & 41.462500 \\
\hline $8 / 28 / 0914: 25$ & $14: 28$ & 5714150 & Normal & OK & Normal $\left(23^{\circ} \mathrm{C}\right)$ & Normal (56\%) & OK & $\mathrm{OK}$ & 139 & -88.371667 & 41.417500 \\
\hline $8 / 28 / 0914: 32$ & $14: 39$ & 5714150 & Normal & OK & Normal $\left(23^{\circ} \mathrm{C}\right)$ & Normal (56\%) & OK & OK & 139 & -88.422778 & 41.392500 \\
\hline $8 / 28 / 0914: 35$ & $14: 39$ & 5714150 & Normal & OK & Normal $\left(23^{\circ} \mathrm{C}\right)$ & Normal $(56 \%)$ & OK & OK & 139 & -88.422778 & 41.392500 \\
\hline $8 / 28 / 0914: 40$ & $14: 44$ & 5714150 & Normal & OK & Normal $\left(23^{\circ} \mathrm{C}\right)$ & Normal (56\%) & OK & OK & 139 & -88.422778 & 41.392500 \\
\hline $8 / 28 / 09$ 14:45 & $14: 50$ & 5714150 & Normal & OK & Normal $\left(23^{\circ} \mathrm{C}\right)$ & Normal (56\%) & OK & OK & 139 & -88.461389 & 41.378056 \\
\hline $8 / 28 / 09$ 14:50 & $14: 56$ & 5714150 & Normal & OK & Normal $\left(23^{\circ} \mathrm{C}\right)$ & Normal (56\%) & OK & OK & 139 & -88.575000 & 41.376944 \\
\hline $8 / 28 / 09$ 14:55 & $15: 01$ & 5714150 & Normal & OK & Normal $\left(23^{\circ} \mathrm{C}\right)$ & Normal (54\%) & OK & OK & 139 & -88.717500 & 41.375833 \\
\hline $8 / 28 / 0915: 00$ & $15: 07$ & 5714150 & Normal & OK & Normal $\left(23^{\circ} \mathrm{C}\right)$ & Normal (54\%) & OK & OK & 139 & -88.792500 & 41.376944 \\
\hline $8 / 28 / 09$ 15:05 & $15: 12$ & 5714150 & Normal & OK & Normal $\left(23^{\circ} \mathrm{C}\right)$ & Normal (54\%) & OK & OK & 139 & -88.901944 & 41.368611 \\
\hline $8 / 28 / 09$ 15:10 & $15: 12$ & 5714150 & Normal & OK & Normal $\left(23^{\circ} \mathrm{C}\right)$ & Normal (54\%) & OK & OK & 139 & -89.014444 & 41.367500 \\
\hline $8 / 28 / 09$ 15:15 & $15: 18$ & 5714150 & Normal & OK & Normal $\left(23^{\circ} \mathrm{C}\right)$ & Normal $(54 \%)$ & OK & OK & 139 & -89.058333 & 41.413333 \\
\hline $8 / 28 / 09$ 15:20 & $15: 23$ & 5714150 & Normal & OK & Normal $\left(23^{\circ} \mathrm{C}\right)$ & Normal $(54 \%)$ & OK & OK & 139 & -89.050833 & 41.460278 \\
\hline $8 / 28 / 0915: 25$ & $15: 29$ & 5714150 & Normal & OK & Normal $\left(23^{\circ} \mathrm{C}\right)$ & Normal (54\%) & OK & OK & 139 & -89.058333 & 41.375833 \\
\hline $8 / 28 / 09$ 15:30 & $15: 35$ & 5714150 & Normal & OK & Normal $\left(23^{\circ} \mathrm{C}\right)$ & Normal (54\%) & OK & OK & 139 & -89.125833 & 41.368611 \\
\hline $8 / 28 / 09$ 15:35 & $15: 40$ & 5714150 & Normal & OK & Normal $\left(23^{\circ} \mathrm{C}\right)$ & Normal (54\%) & OK & OK & 139 & -89.125833 & 41.368611 \\
\hline $8 / 28 / 09$ 15:40 & $15: 46$ & 5714150 & Normal & OK & Normal $\left(23^{\circ} \mathrm{C}\right)$ & Normal (54\%) & OK & OK & 139 & -89.125833 & 41.368611 \\
\hline $8 / 28 / 09$ 15:45 & $\begin{array}{l}15: 40 \\
15: 51\end{array}$ & 5714150 & Normal & OK & Normal $\left(23^{\circ} \mathrm{C}\right)$ & Normal $(54 \%)$ & OK & OK & $\begin{array}{l}135 \\
139\end{array}$ & -89.200000 & 41.364444 \\
\hline $8 / 28 / 0915: 50$ & $15: 57$ & 5714150 & Normal & OK & Normal $\left(23^{\circ} \mathrm{C}\right)$ & Normal (54\%) & OK & OK & 139 & -89.240556 & 41.371667 \\
\hline $8 / 28 / 0916: 00$ & $16: 03$ & 5714150 & Normal & OK & Normal $\left(23^{\circ} \mathrm{C}\right)$ & Normal (54\%) & OK & OK & 139 & -89.458333 & 41.400833 \\
\hline $8 / 28 / 0916: 05$ & $16: 13$ & 5714150 & Normal & OK & Normal $\left(23^{\circ} \mathrm{C}\right)$ & Normal ( $54 \%$ ) & OK & $\mathrm{OK}$ & 139 & -89.558333 & 41.393611 \\
\hline
\end{tabular}




\begin{tabular}{|c|c|c|c|c|c|c|c|c|c|c|c|}
\hline Event Time & at QC Hub & Tag ID & Tag Status & Seal & Temperature & Humidity & Shock & Battery & RSSI & Longitude & Latitude \\
\hline 8/28/09 16:11 & $16: 14$ & 5714150 & Normal & OK & Normal $\left(23^{\circ} \mathrm{C}\right)$ & Normal $(54 \%)$ & OK & OK & 139 & -89.558333 & 41.393611 \\
\hline $8 / 28 / 09$ 16:15 & $16: 19$ & 5714150 & Normal & OK & Normal $\left(23^{\circ} \mathrm{C}\right)$ & Normal (54\%) & OK & OK & 139 & -89.558333 & 41.393611 \\
\hline $8 / 28 / 0916: 20$ & $16: 25$ & 5714150 & Normal & OK & Normal $\left(23^{\circ} \mathrm{C}\right)$ & Normal (54\%) & OK & OK & 139 & -89.558333 & 41.393611 \\
\hline $8 / 28 / 0916: 25$ & $16: 30$ & 5714150 & Normal & OK & Normal $\left(23^{\circ} \mathrm{C}\right)$ & Normal (54\%) & OK & OK & 139 & -89.623889 & 41.394722 \\
\hline $8 / 28 / 09$ 16:30 & $16: 36$ & 5714150 & Normal & OK & Normal $\left(23^{\circ} \mathrm{C}\right)$ & Normal (54\%) & OK & OK & 139 & -89.725833 & 41.387500 \\
\hline $8 / 28 / 09$ 16:35 & $16: 41$ & 5714150 & Normal & OK & Normal $\left(23^{\circ} \mathrm{C}\right)$ & Normal (54\%) & OK & OK & 139 & -89.834167 & 41.388333 \\
\hline $8 / 28 / 09$ 16:40 & $16: 47$ & 5714150 & Normal & OK & Normal $\left(23^{\circ} \mathrm{C}\right)$ & Normal (54\%) & OK & OK & 139 & -89.941667 & 41.416667 \\
\hline 8/28/09 16:45 & $16: 52$ & 5714150 & Normal & OK & Normal $\left(23^{\circ} \mathrm{C}\right)$ & Normal (54\%) & OK & OK & 139 & -90.050000 & 41.413333 \\
\hline $8 / 28 / 09$ 16:50 & $16: 52$ & 5714150 & Normal & OK & Normal $\left(23^{\circ} \mathrm{C}\right)$ & Normal (54\%) & OK & OK & 139 & -90.157222 & 41.430000 \\
\hline 8/28/09 16:55 & $16: 58$ & 5714150 & Normal & OK & Normal $\left(23^{\circ} \mathrm{C}\right)$ & Normal (54\%) & OK & OK & 139 & -90.267500 & 41.440556 \\
\hline 8/28/09 17:00 & 17:09 & 5714150 & Normal & OK & Normal $\left(23^{\circ} \mathrm{C}\right)$ & Normal (54\%) & OK & $\mathrm{OK}$ & 139 & -90.338333 & 41.476944 \\
\hline 8/28/09 17:05 & 17:09 & 5714150 & Normal & OK & Normal $\left(23^{\circ} \mathrm{C}\right)$ & Normal (54\%) & OK & OK & 139 & -90.334167 & 41.486389 \\
\hline 8/28/09 17:11 & $17: 15$ & 5714150 & Normal & OK & Normal $\left(23^{\circ} \mathrm{C}\right)$ & Normal (54\%) & OK & OK & 139 & -90.334167 & 41.486389 \\
\hline 8/28/09 17:15 & $17: 20$ & 5714150 & Normal & OK & Normal $\left(23^{\circ} \mathrm{C}\right)$ & Normal (54\%) & OK & OK & 139 & -90.334167 & 41.486389 \\
\hline $8 / 28 / 0917: 20$ & $17: 26$ & 5714150 & Normal & OK & Normal $\left(23^{\circ} \mathrm{C}\right)$ & Normal (54\%) & OK & OK & 139 & -90.334167 & 41.486389 \\
\hline 8/28/09 17:25 & $17: 31$ & 5714150 & Normal & OK & Normal $\left(23^{\circ} \mathrm{C}\right)$ & Normal (54\%) & OK & OK & 139 & -90.340556 & 41.511389 \\
\hline $8 / 28 / 09$ 17:30 & $17: 37$ & 5714150 & Normal & OK & Normal $\left(23^{\circ} \mathrm{C}\right)$ & Normal (54\%) & OK & OK & 139 & -90.234167 & 41.562500 \\
\hline 8/28/09 17:35 & $17: 42$ & 5714150 & Normal & OK & Normal $\left(23^{\circ} \mathrm{C}\right)$ & Normal (54\%) & OK & OK & 139 & -90.209167 & 41.581111 \\
\hline 8/28/09 17:40 & $17: 42$ & 5714150 & Normal & OK & Normal $\left(23^{\circ} \mathrm{C}\right)$ & Normal (54\%) & OK & $\mathrm{OK}$ & 139 & -90.163333 & 41.642500 \\
\hline $8 / 28 / 09$ 17:45 & $17: 48$ & 5714150 & Normal & OK & Normal $\left(23^{\circ} \mathrm{C}\right)$ & Normal (54\%) & OK & OK & 139 & -90.080000 & 41.690556 \\
\hline $8 / 28 / 09$ 17:50 & $17: 53$ & 5714150 & Normal & OK & Normal $\left(23^{\circ} \mathrm{C}\right)$ & Normal $(54 \%)$ & OK & OK & 139 & -89.983333 & 41.718611 \\
\hline 8/28/09 17:55 & $17: 59$ & 5714150 & Normal & OK & Normal $\left(25^{\circ} \mathrm{C}\right)$ & Normal (54\%) & OK & OK & 139 & -89.876944 & 41.744722 \\
\hline 8/28/09 18:00 & $18: 04$ & 5714150 & Normal & OK & Normal $\left(25^{\circ} \mathrm{C}\right)$ & Normal (54\%) & OK & OK & 139 & -89.775000 & 41.757222 \\
\hline $8 / 28 / 0918: 05$ & $18: 10$ & 5714150 & Normal & OK & Normal $\left(25^{\circ} \mathrm{C}\right)$ & Normal (54\%) & OK & OK & 139 & -89.689444 & 41.750833 \\
\hline 8/28/09 18:10 & $18: 15$ & 5714150 & Normal & OK & Normal $\left(25^{\circ} \mathrm{C}\right)$ & Normal (54\%) & OK & OK & 139 & -89.689444 & 41.762500 \\
\hline 8/28/09 18:15 & $18: 21$ & 5714150 & Normal & OK & Normal $\left(25^{\circ} \mathrm{C}\right)$ & Normal (54\%) & OK & OK & 139 & -89.678056 & 41.750833 \\
\hline $8 / 28 / 0918: 20$ & $18: 26$ & 5714150 & Normal & OK & Normal $\left(25^{\circ} \mathrm{C}\right)$ & Normal (54\%) & OK & OK & 139 & -89.635278 & 41.759167 \\
\hline $8 / 28 / 09$ 18:25 & $18: 32$ & 5714150 & Normal & OK & Normal $\left(25^{\circ} \mathrm{C}\right)$ & Normal $(54 \%)$ & OK & OK & 139 & -89.532222 & 41.789444 \\
\hline $8 / 28 / 0918: 30$ & $18: 38$ & 5714150 & Normal & OK & Normal $\left(25^{\circ} \mathrm{C}\right)$ & Normal (54\%) & OK & OK & 139 & -89.432222 & 41.822778 \\
\hline $8 / 28 / 0918: 35$ & $18: 38$ & 5714150 & Normal & OK & Normal $\left(25^{\circ} \mathrm{C}\right)$ & Normal (54\%) & OK & OK & 139 & -89.336389 & 41.822778 \\
\hline $8 / 28 / 0918: 40$ & $18: 43$ & 5714150 & Normal & OK & Normal $\left(25^{\circ} \mathrm{C}\right)$ & Normal $(54 \%)$ & OK & OK & 139 & -89.231111 & 41.850833 \\
\hline $8 / 28 / 0918: 45$ & $18: 48$ & 5714150 & Normal & OK & Normal $\left(25^{\circ} \mathrm{C}\right)$ & Normal (54\%) & OK & OK & 139 & -89.125000 & 41.883333 \\
\hline $8 / 28 / 0918: 50$ & $18: 54$ & 5714150 & Normal & OK & Normal $\left(25^{\circ} \mathrm{C}\right)$ & Normal $(54 \%)$ & OK & OK & 139 & -89.025000 & 41.904167 \\
\hline $8 / 28 / 0918: 55$ & $19: 00$ & 5714150 & Normal & OK & Normal $\left(25^{\circ} \mathrm{C}\right)$ & Normal (54\%) & OK & OK & 139 & -88.913333 & 41.900000 \\
\hline $8 / 28 / 0919: 00$ & 19:05 & 5714150 & Normal & OK & Normal $\left(25^{\circ} \mathrm{C}\right)$ & Normal (54\%) & OK & OK & 139 & -88.814444 & 41.900000 \\
\hline $8 / 28 / 09$ 19:05 & 19:11 & 5714150 & Normal & OK & Normal $\left(25^{\circ} \mathrm{C}\right)$ & Normal (54\%) & OK & OK & 139 & -88.739444 & 41.900000 \\
\hline 8/28/09 19:10 & $19: 16$ & 5714150 & Normal & OK & Normal $\left(25^{\circ} \mathrm{C}\right)$ & Normal (54\%) & OK & OK & 139 & -88.739444 & 41.900000 \\
\hline 8/28/09 19:15 & $19: 22$ & 5714150 & Normal & OK & Normal $\left(25^{\circ} \mathrm{C}\right)$ & Normal (54\%) & OK & OK & 139 & -88.737500 & 41.900000 \\
\hline $8 / 28 / 09$ 19:20 & $19: 27$ & 5714150 & Normal & OK & Normal $\left(25^{\circ} \mathrm{C}\right)$ & Normal (54\%) & OK & OK & 139 & -88.737500 & 41.900000 \\
\hline $8 / 28 / 09$ 19:25 & $19: 33$ & 5714150 & Normal & OK & Normal $\left(25^{\circ} \mathrm{C}\right)$ & Normal $(54 \%)$ & OK & OK & 139 & -88.639444 & 41.894722 \\
\hline $8 / 28 / 09$ 19:30 & $19: 38$ & 5714150 & Normal & OK & Normal $\left(25^{\circ} \mathrm{C}\right)$ & Normal (54\%) & OK & OK & 139 & -88.538333 & 41.859167 \\
\hline $8 / 28 / 09$ 19:35 & $19: 38$ & 5714150 & Normal & OK & Normal $\left(25^{\circ} \mathrm{C}\right)$ & Normal $(54 \%)$ & OK & OK & 139 & -88.448889 & 41.812500 \\
\hline $8 / 28 / 09$ 19:40 & $19: 44$ & 5714150 & Normal & OK & Normal $\left(25^{\circ} \mathrm{C}\right)$ & Normal (54\%) & OK & OK & 139 & -88.347778 & 41.794722 \\
\hline $8 / 28 / 09$ 19:45 & $19: 49$ & 5714150 & Normal & OK & Normal $\left(25^{\circ} \mathrm{C}\right)$ & Normal (54\%) & OK & OK & 139 & -88.261389 & 41.800000 \\
\hline 8/28/09 19:50 & 19:55 & 5714150 & Normal & OK & Normal $\left(25^{\circ} \mathrm{C}\right)$ & Normal (54\%) & OK & $\mathrm{OK}$ & 139 & -88.156111 & 41.806111 \\
\hline 8/28/09 19:55 & 20:00 & 5714150 & Normal & OK & Normal $\left(25^{\circ} \mathrm{C}\right)$ & Normal (54\%) & OK & OK & 139 & -88.062500 & 41.810278 \\
\hline $8 / 28 / 09$ 20:00 & 20:06 & 5714150 & Normal & OK & Normal $\left(25^{\circ} \mathrm{C}\right)$ & Normal $(54 \%)$ & OK & OK & 139 & -88.054167 & 41.807222 \\
\hline $8 / 28 / 0920: 05$ & 20:11 & 5714150 & Normal & OK & Normal $\left(25^{\circ} \mathrm{C}\right)$ & Normal $(54 \%)$ & OK & OK & 139 & -88.054167 & 41.781111 \\
\hline 8/28/09 20:10 & $20: 22$ & 5714150 & Normal & OK & Normal $\left(25^{\circ} \mathrm{C}\right)$ & Normal $(54 \%)$ & OK & OK & 139 & -88.035278 & 41.732222 \\
\hline $8 / 28 / 0913: 40$ & $13: 44$ & 5714155 & Normal & OK & $\operatorname{Normal}\left(23^{\circ} \mathrm{C}\right)$ & Normal $(56 \%)$ & $\overline{O K}$ & OK & 138 & -87.971667 & 41.732222 \\
\hline $8 / 28 / 09$ 13:45 & $13: 49$ & 5714155 & Normal & OK & Normal $\left(23^{\circ} \mathrm{C}\right)$ & Normal (56\%) & OK & OK & 138 & -87.971667 & 41.732222 \\
\hline $8 / 28 / 0913: 50$ & $14: 00$ & 5714155 & Normal & OK & Normal $\left(23^{\circ} \mathrm{C}\right)$ & Normal (56\%) & OK & OK & 138 & -87.971667 & 41.732222 \\
\hline 8/28/09 13:55 & $14: 00$ & 5714155 & Normal & OK & Normal $\left(23^{\circ} \mathrm{C}\right)$ & Normal (56\%) & OK & OK & 138 & -87.971667 & 41.732222 \\
\hline $8 / 28 / 0914: 00$ & $14: 06$ & 5714155 & Normal & OK & Normal $\left(23^{\circ} \mathrm{C}\right)$ & Normal $(56 \%)$ & OK & OK & 138 & -88.068611 & 41.684167 \\
\hline $8 / 28 / 0914: 05$ & $14: 11$ & 5714155 & Normal & OK & Normal $\left(23^{\circ} \mathrm{C}\right)$ & Normal $(56 \%)$ & OK & OK & 138 & -88.154167 & 41.640556 \\
\hline $8 / 28 / 0914: 10$ & $14: 17$ & 5714155 & Normal & OK & Normal $\left(23^{\circ} \mathrm{C}\right)$ & Normal $(56 \%)$ & OK & OK & 138 & -88.181111 & 41.568611 \\
\hline 8/28/09 14:15 & $14: 22$ & 5714155 & Normal & OK & Normal $\left(23^{\circ} \mathrm{C}\right)$ & Normal (56\%) & OK & OK & 138 & -88.197778 & 41.493611 \\
\hline $8 / 28 / 0914: 20$ & $14: 22$ & 5714155 & Normal & OK & Normal $\left(23^{\circ} \mathrm{C}\right)$ & Normal $(56 \%)$ & OK & OK & 138 & -88.280000 & 41.462500 \\
\hline $8 / 28 / 09$ 14:25 & $14: 28$ & 5714155 & Normal & OK & Normal $\left(23^{\circ} \mathrm{C}\right)$ & Normal (56\%) & OK & OK & 138 & -88.371667 & 41.417500 \\
\hline $8 / 28 / 09$ 14:32 & $14: 39$ & 5714155 & Normal & OK & Normal $\left(23^{\circ} \mathrm{C}\right)$ & Normal $(56 \%)$ & OK & OK & 138 & -88.422778 & 41.392500 \\
\hline $8 / 28 / 09$ 14:35 & $14: 39$ & 5714155 & Normal & OK & Normal $\left(23^{\circ} \mathrm{C}\right)$ & Normal (56\%) & OK & OK & 138 & -88.422778 & 41.392500 \\
\hline $8 / 28 / 0914: 40$ & $14: 44$ & 5714155 & Normal & OK & Normal $\left(23^{\circ} \mathrm{C}\right)$ & Normal $(56 \%)$ & OK & OK & 138 & -88.422778 & 41.392500 \\
\hline $8 / 28 / 09$ 14:45 & $14: 50$ & 5714155 & Normal & OK & Normal $\left(23^{\circ} \mathrm{C}\right)$ & Normal $(56 \%)$ & OK & OK & 138 & -88.461389 & 41.378056 \\
\hline $8 / 28 / 0914: 50$ & $14: 56$ & 5714155 & Normal & OK & Normal $\left(23^{\circ} \mathrm{C}\right)$ & Normal $(56 \%)$ & OK & OK & 138 & -88.575000 & 41.376944 \\
\hline $8 / 28 / 0914: 55$ & $15: 01$ & 5714155 & Normal & OK & Normal $\left(23^{\circ} \mathrm{C}\right)$ & Normal (54\%) & OK & OK & 138 & -88.717500 & 41.375833 \\
\hline $8 / 28 / 09$ 15:00 & $15: 07$ & 5714155 & Normal & OK & Normal $\left(23^{\circ} \mathrm{C}\right)$ & Normal (54\%) & OK & OK & 138 & -88.792500 & 41.376944 \\
\hline $8 / 28 / 09$ 15:05 & $15: 12$ & 5714155 & Normal & OK & Normal $\left(23^{\circ} \mathrm{C}\right)$ & Normal (54\%) & OK & OK & 138 & -88.901944 & 41.368611 \\
\hline $8 / 28 / 09$ 15:10 & $15: 12$ & 5714155 & Normal & OK & Normal $\left(23^{\circ} \mathrm{C}\right)$ & Normal (54\%) & OK & OK & 138 & -89.014444 & 41.367500 \\
\hline $8 / 28 / 09$ 15:15 & $15: 18$ & 5714155 & Normal & OK & Normal $\left(23^{\circ} \mathrm{C}\right)$ & Normal (54\%) & OK & OK & 138 & -89.058333 & 41.413333 \\
\hline $8 / 28 / 09$ 15:20 & $15: 23$ & 5714155 & Normal & OK & Normal $\left(23^{\circ} \mathrm{C}\right)$ & Normal (54\%) & OK & OK & 138 & -89.050833 & 41.460278 \\
\hline $8 / 28 / 09$ 15:25 & $15: 29$ & 5714155 & Normal & OK & Normal $\left(23^{\circ} \mathrm{C}\right)$ & Normal (54\%) & OK & OK & 138 & -89.058333 & 41.375833 \\
\hline $8 / 28 / 09$ 15:30 & $15: 35$ & 5714155 & Normal & OK & Normal $\left(23^{\circ} \mathrm{C}\right)$ & Normal (54\%) & OK & OK & 138 & -89.125833 & 41.368611 \\
\hline $8 / 28 / 0915: 35$ & $15: 40$ & 5714155 & Normal & OK & Normal $\left(23^{\circ} \mathrm{C}\right)$ & Normal (54\%) & OK & OK & 138 & -89.125833 & 41.368611 \\
\hline $8 / 28 / 0915: 40$ & $15: 46$ & 5714155 & Normal & OK & Normal $\left(23^{\circ} \mathrm{C}\right)$ & Normal $(54 \%)$ & OK & OK & 138 & -89.125833 & 41.368611 \\
\hline $8 / 28 / 09$ 15:45 & $15: 51$ & 5714155 & Normal & OK & Normal $\left(23^{\circ} \mathrm{C}\right)$ & Normal (54\%) & OK & OK & 138 & -89.200000 & 41.364444 \\
\hline $8 / 28 / 0915: 50$ & $15: 57$ & 5714155 & Normal & OK & Normal $\left(23^{\circ} \mathrm{C}\right)$ & Normal (54\%) & OK & OK & 138 & -89.240556 & 41.371667 \\
\hline $8 / 28 / 0916: 00$ & $16: 03$ & 5714155 & Normal & OK & Normal $\left(23^{\circ} \mathrm{C}\right)$ & Normal (54\%) & OK & OK & 138 & -89.458333 & 41.400833 \\
\hline $8 / 28 / 0916: 05$ & $16: 13$ & 5714155 & Normal & OK & Normal $\left(23^{\circ} \mathrm{C}\right)$ & Normal (54\%) & OK & OK & 138 & -89.558333 & 41.393611 \\
\hline $8 / 28 / 09$ 16:11 & $16: 14$ & 5714155 & Normal & OK & Normal $\left(23^{\circ} \mathrm{C}\right)$ & Normal (54\%) & OK & OK & 138 & -89.558333 & 41.393611 \\
\hline $8 / 28 / 0916: 15$ & $16: 19$ & 5714155 & Normal & OK & Normal $\left(23^{\circ} \mathrm{C}\right)$ & Normal (54\%) & OK & OK & 138 & -89.558333 & 41.393611 \\
\hline $8 / 28 / 09$ 16:20 & $16: 25$ & 5714155 & Normal & OK & Normal $\left(23^{\circ} \mathrm{C}\right)$ & Normal (54\%) & OK & OK & 138 & -89.558333 & 41.393611 \\
\hline
\end{tabular}




\begin{tabular}{|c|c|c|c|c|c|c|c|c|c|c|c|}
\hline Event Time & at QC Hub & Tag ID & Tag Status & Seal & Temperature & Humidity & Shock & Battery & RSSI & Longitude & Latitude \\
\hline $8 / 28 / 0916: 25$ & $16: 30$ & 5714155 & Normal & OK & Normal $\left(23^{\circ} \mathrm{C}\right)$ & Normal (54\%) & OK & OK & 138 & -89.623889 & 41.394722 \\
\hline $8 / 28 / 09$ 16:30 & $16: 36$ & 5714155 & Normal & OK & Normal $\left(23^{\circ} \mathrm{C}\right)$ & Normal (54\%) & $\mathrm{OK}$ & OK & 138 & -89.725833 & 41.387500 \\
\hline $8 / 28 / 09$ 16:35 & $16: 41$ & 5714155 & Normal & OK & Normal $\left(23^{\circ} \mathrm{C}\right)$ & Normal (54\%) & OK & OK & 138 & -89.834167 & 41.388333 \\
\hline $8 / 28 / 0916: 40$ & $16: 47$ & 5714155 & Normal & OK & Normal $\left(23^{\circ} \mathrm{C}\right)$ & Normal (54\%) & OK & $\mathrm{OK}$ & 138 & -89.941667 & 41.416667 \\
\hline $8 / 28 / 0916: 45$ & $16: 52$ & 5714155 & Normal & OK & Normal $\left(23^{\circ} \mathrm{C}\right)$ & Normal (54\%) & OK & OK & 138 & -90.050000 & 41.413333 \\
\hline $8 / 28 / 09$ 16:50 & $16: 52$ & 5714155 & Normal & OK & Normal $\left(23^{\circ} \mathrm{C}\right)$ & Normal (54\%) & OK & OK & 138 & -90.157222 & 41.430000 \\
\hline $8 / 28 / 09$ 16:55 & $16: 58$ & 5714155 & Normal & OK & Normal $\left(23^{\circ} \mathrm{C}\right)$ & Normal (54\%) & OK & ок & 138 & -90.267500 & 41.440556 \\
\hline $8 / 28 / 09$ 17:00 & 17:09 & 5714155 & Normal & OK & Normal $\left(23^{\circ} \mathrm{C}\right)$ & Normal (54\%) & OK & OK & 138 & -90.338333 & 41.476944 \\
\hline $8 / 28 / 09$ 17:05 & 17:09 & 5714155 & Normal & OK & Normal $\left(23^{\circ} \mathrm{C}\right)$ & Normal (54\%) & OK & OK & 138 & -90.334167 & 41.486389 \\
\hline $8 / 28 / 09$ 17:11 & $17: 15$ & 5714155 & Normal & OK & Normal $\left(23^{\circ} \mathrm{C}\right)$ & Normal (54\%) & OK & OK & 138 & -90.334167 & 41.486389 \\
\hline $8 / 28 / 09$ 17:15 & $17: 20$ & 5714155 & Normal & OK & Normal $\left(23^{\circ} \mathrm{C}\right)$ & Normal (54\%) & OK & OK & 138 & -90.334167 & 41.486389 \\
\hline $8 / 28 / 09$ 17:20 & $17: 26$ & 5714155 & Normal & OK & Normal $\left(23^{\circ} \mathrm{C}\right)$ & Normal (54\%) & OK & OK & 138 & -90.334167 & 41.486389 \\
\hline $8 / 28 / 09$ 17:25 & $17: 31$ & 5714155 & Normal & OK & Normal $\left(23^{\circ} \mathrm{C}\right)$ & Normal (54\%) & OK & OK & 138 & -90.340556 & 41.511389 \\
\hline $8 / 28 / 09$ 17:30 & $17: 37$ & 5714155 & Normal & OK & Normal $\left(23^{\circ} \mathrm{C}\right)$ & Normal (54\%) & OK & OK & 138 & -90.234167 & 41.562500 \\
\hline $8 / 28 / 09$ 17:35 & $17: 42$ & 5714155 & Normal & OK & Normal $\left(23^{\circ} \mathrm{C}\right)$ & Normal (54\%) & OK & OK & 138 & -90.209167 & 41.581111 \\
\hline $8 / 28 / 0917: 40$ & $17: 42$ & 5714155 & Normal & OK & Normal $\left(23^{\circ} \mathrm{C}\right)$ & Normal (54\%) & OK & OK & 138 & -90.163333 & 41.642500 \\
\hline $8 / 28 / 09$ 17:45 & $17: 48$ & 5714155 & Normal & OK & Normal $\left(23^{\circ} \mathrm{C}\right)$ & Normal (54\%) & OK & OK & 138 & -90.080000 & 41.690556 \\
\hline $8 / 28 / 09$ 17:50 & $17: 53$ & 5714155 & Normal & OK & Normal $\left(23^{\circ} \mathrm{C}\right)$ & Normal (54\%) & OK & OK & 138 & -89.983333 & 41.718611 \\
\hline $8 / 28 / 09$ 17:55 & $17: 59$ & 5714155 & Normal & OK & Normal $\left(25^{\circ} \mathrm{C}\right)$ & Normal (54\%) & OK & OK & 138 & -89.876944 & 41.744722 \\
\hline $8 / 28 / 0918: 00$ & $18: 04$ & 5714155 & Normal & OK & Normal $\left(25^{\circ} \mathrm{C}\right)$ & Normal (54\%) & OK & OK & 138 & -89.775000 & 41.757222 \\
\hline $8 / 28 / 09$ 18:05 & $18: 10$ & 5714155 & Normal & OK & Normal $\left(25^{\circ} \mathrm{C}\right)$ & Normal (54\%) & OK & OK & 138 & -89.689444 & 41.750833 \\
\hline $8 / 28 / 09$ 18:10 & $18: 15$ & 5714155 & Normal & OK & Normal $\left(25^{\circ} \mathrm{C}\right)$ & Normal (54\%) & OK & OK & 138 & -89.689444 & 41.762500 \\
\hline $8 / 28 / 09$ 18:15 & $18: 21$ & 5714155 & Normal & OK & Normal $\left(25^{\circ} \mathrm{C}\right)$ & Normal (54\%) & OK & OK & 138 & -89.678056 & 41.750833 \\
\hline $8 / 28 / 0918: 20$ & $18: 26$ & 5714155 & Normal & OK & Normal $\left(25^{\circ} \mathrm{C}\right)$ & Normal (54\%) & OK & OK & 138 & -89.635278 & 41.759167 \\
\hline $8 / 28 / 0918: 25$ & $18: 32$ & 5714155 & Normal & OK & Normal $\left(25^{\circ} \mathrm{C}\right)$ & Normal (54\%) & OK & OK & 138 & -89.532222 & 41.789444 \\
\hline $8 / 28 / 09$ 18:30 & $18: 38$ & 5714155 & Normal & OK & Normal $\left(25^{\circ} \mathrm{C}\right)$ & Normal (54\%) & OK & OK & 138 & -89.432222 & 41.822778 \\
\hline $8 / 28 / 09$ 18:35 & $18: 38$ & 5714155 & Normal & OK & Normal $\left(25^{\circ} \mathrm{C}\right)$ & Normal (54\%) & OK & OK & 138 & -89.336389 & 41.822778 \\
\hline $8 / 28 / 09$ 18:40 & $18: 43$ & 5714155 & Normal & OK & Normal $\left(25^{\circ} \mathrm{C}\right)$ & Normal (54\%) & OK & OK & 138 & -89.231111 & 41.850833 \\
\hline $8 / 28 / 09$ 18:45 & $18: 48$ & 5714155 & Normal & OK & Normal $\left(25^{\circ} \mathrm{C}\right)$ & Normal (54\%) & OK & OK & 138 & -89.125000 & 41.883333 \\
\hline $8 / 28 / 09$ 18:50 & $18: 54$ & 5714155 & Normal & OK & Normal $\left(25^{\circ} \mathrm{C}\right)$ & Normal (54\%) & OK & OK & 138 & -89.025000 & 41.904167 \\
\hline $8 / 28 / 09$ 18:55 & $19: 00$ & 5714155 & Normal & OK & Normal $\left(25^{\circ} \mathrm{C}\right)$ & Normal (54\%) & OK & OK & 138 & -88.913333 & 41.900000 \\
\hline $8 / 28 / 09$ 19:00 & $19: 05$ & 5714155 & Normal & OK & Normal $\left(25^{\circ} \mathrm{C}\right)$ & Normal (54\%) & OK & OK & 138 & -88.814444 & 41.900000 \\
\hline $8 / 28 / 09$ 19:05 & $19: 11$ & 5714155 & Normal & OK & Normal $\left(25^{\circ} \mathrm{C}\right)$ & Normal (54\%) & OK & OK & 138 & -88.739444 & 41.900000 \\
\hline 8/28/09 19:10 & $19: 16$ & 5714155 & Normal & OK & Normal $\left(25^{\circ} \mathrm{C}\right)$ & Normal (54\%) & OK & OK & 138 & -88.739444 & 41.900000 \\
\hline $8 / 28 / 09$ 19:15 & $19: 22$ & 5714155 & Normal & OK & Normal $\left(25^{\circ} \mathrm{C}\right)$ & Normal (53\%) & OK & OK & 138 & -88.737500 & 41.900000 \\
\hline $8 / 28 / 09$ 19:20 & $19: 27$ & 5714155 & Normal & OK & Normal $\left(25^{\circ} \mathrm{C}\right)$ & Normal (53\%) & OK & OK & 138 & -88.737500 & 41.900000 \\
\hline $8 / 28 / 09$ 19:25 & $19: 33$ & 5714155 & Normal & OK & Normal $\left(25^{\circ} \mathrm{C}\right)$ & Normal (53\%) & OK & OK & 138 & -88.639444 & 41.894722 \\
\hline $8 / 28 / 09$ 19:30 & $19: 38$ & 5714155 & Normal & OK & Normal $\left(25^{\circ} \mathrm{C}\right)$ & Normal (53\%) & OK & OK & 138 & -88.538333 & 41.859167 \\
\hline $8 / 28 / 09$ 19:35 & $19: 38$ & 5714155 & Normal & OK & Normal $\left(25^{\circ} \mathrm{C}\right)$ & Normal (53\%) & OK & OK & 138 & -88.448889 & 41.812500 \\
\hline $8 / 28 / 0919: 40$ & $19: 44$ & 5714155 & Normal & OK & Normal $\left(25^{\circ} \mathrm{C}\right)$ & Normal (53\%) & OK & OK & 138 & -88.347778 & 41.794722 \\
\hline $8 / 28 / 09$ 19:45 & $19: 49$ & 5714155 & Normal & OK & Normal $\left(25^{\circ} \mathrm{C}\right)$ & Normal (53\%) & OK & OK & 138 & -88.261389 & 41.800000 \\
\hline $8 / 28 / 09$ 19:50 & $19: 55$ & 5714155 & Normal & OK & Normal $\left(25^{\circ} \mathrm{C}\right)$ & Normal (53\%) & OK & OK & 138 & -88.156111 & 41.806111 \\
\hline $8 / 28 / 09$ 19:55 & $20: 00$ & 5714155 & Normal & OK & Normal $\left(25^{\circ} \mathrm{C}\right)$ & Normal (53\%) & OK & OK & 138 & -88.062500 & 41.810278 \\
\hline $8 / 28 / 0920: 00$ & $20: 06$ & 5714155 & Normal & OK & Normal $\left(25^{\circ} \mathrm{C}\right)$ & Normal (53\%) & OK & OK & 138 & -88.054167 & 41.807222 \\
\hline 8/28/09 20:05 & $20: 11$ & 5714155 & Normal & OK & Normal $\left(25^{\circ} \mathrm{C}\right)$ & Normal (54\%) & OK & OK & 138 & -88.054167 & 41.781111 \\
\hline $8 / 28 / 0920: 10$ & $20: 22$ & 5714155 & Normal & OK & Normal $\left(25^{\circ} \mathrm{C}\right)$ & Normal $(54 \%)$ & OK & $\mathrm{OK}$ & 138 & -88.035278 & 41.732222 \\
\hline $8 / 28 / 09$ 13:40 & $13: 44$ & 5714160 & Normal & $\overline{O K}$ & Normal $\left(23^{\circ} \mathrm{C}\right)$ & Normal $(56 \%)$ & $\overline{O K}$ & $\overline{\mathrm{OK}}$ & 135 & -87.971667 & 41.732222 \\
\hline $8 / 28 / 0913: 45$ & $13: 49$ & 5714160 & Normal & OK & Normal $\left(23^{\circ} \mathrm{C}\right)$ & Normal $(56 \%)$ & OK & OK & 135 & -87.971667 & 41.732222 \\
\hline $8 / 28 / 0913: 50$ & $14: 00$ & 5714160 & Normal & OK & Normal $\left(23^{\circ} \mathrm{C}\right)$ & Normal $(56 \%)$ & OK & OK & 135 & -87.971667 & 41.732222 \\
\hline $8 / 28 / 0913: 55$ & $14: 00$ & 5714160 & Normal & OK & Normal $\left(23^{\circ} \mathrm{C}\right)$ & Normal $(56 \%)$ & OK & OK & 135 & -87.971667 & 41.732222 \\
\hline $8 / 28 / 0914: 00$ & $14: 06$ & 5714160 & Normal & OK & Normal $\left(23^{\circ} \mathrm{C}\right)$ & Normal $(56 \%)$ & $\mathrm{OK}$ & OK & 135 & -88.068611 & 41.684167 \\
\hline $8 / 28 / 0914: 05$ & $14: 11$ & 5714160 & Normal & OK & Normal $\left(23^{\circ} \mathrm{C}\right)$ & Normal (56\%) & OK & OK & 135 & -88.154167 & 41.640556 \\
\hline $8 / 28 / 0914: 10$ & $14: 17$ & 5714160 & Normal & OK & Normal $\left(23^{\circ} \mathrm{C}\right)$ & Normal $(56 \%)$ & OK & OK & 135 & -88.181111 & 41.568611 \\
\hline $8 / 28 / 0914: 15$ & $14: 22$ & 5714160 & Normal & OK & Normal $\left(23^{\circ} \mathrm{C}\right)$ & Normal (56\%) & OK & OK & 135 & -88.197778 & 41.493611 \\
\hline $8 / 28 / 0914: 20$ & $14: 22$ & 5714160 & Normal & OK & Normal $\left(23^{\circ} \mathrm{C}\right)$ & Normal $(56 \%)$ & OK & OK & 135 & -88.280000 & 41.462500 \\
\hline $8 / 28 / 0914: 25$ & $14: 28$ & 5714160 & Normal & OK & Normal $\left(23^{\circ} \mathrm{C}\right)$ & Normal $(56 \%)$ & OK & OK & 135 & -88.371667 & 41.417500 \\
\hline $8 / 28 / 0914: 32$ & $14: 39$ & 5714160 & Normal & OK & Normal $\left(23^{\circ} \mathrm{C}\right)$ & Normal (56\%) & OK & OK & 135 & -88.422778 & 41.392500 \\
\hline $8 / 28 / 0914: 35$ & $14: 39$ & 5714160 & Normal & OK & Normal $\left(23^{\circ} \mathrm{C}\right)$ & Normal (56\%) & OK & OK & 135 & -88.422778 & 41.392500 \\
\hline $8 / 28 / 0914: 40$ & $14: 44$ & 5714160 & Normal & OK & Normal $\left(23^{\circ} \mathrm{C}\right)$ & Normal (56\%) & OK & OK & 135 & -88.422778 & 41.392500 \\
\hline $8 / 28 / 09$ 14:45 & $14: 50$ & 5714160 & Normal & OK & Normal $\left(23^{\circ} \mathrm{C}\right)$ & Normal (56\%) & OK & OK & 135 & -88.461389 & 41.378056 \\
\hline $8 / 28 / 0914: 50$ & $14: 56$ & 5714160 & Normal & OK & Normal $\left(23^{\circ} \mathrm{C}\right)$ & Normal $(56 \%)$ & OK & OK & 135 & -88.575000 & 41.376944 \\
\hline $8 / 28 / 0914: 55$ & $15: 01$ & 5714160 & Normal & OK & Normal $\left(23^{\circ} \mathrm{C}\right)$ & Normal $(56 \%)$ & OK & OK & 135 & -88.717500 & 41.375833 \\
\hline $8 / 28 / 0915: 00$ & $15: 07$ & 5714160 & Normal & OK & Normal $\left(23^{\circ} \mathrm{C}\right)$ & Normal $(56 \%)$ & OK & OK & 135 & -88.792500 & 41.376944 \\
\hline $8 / 28 / 09$ 15:05 & $15: 12$ & 5714160 & Normal & OK & Normal $\left(23^{\circ} \mathrm{C}\right)$ & Normal $(56 \%)$ & OK & OK & 135 & -88.901944 & 41.368611 \\
\hline $8 / 28 / 09$ 15:10 & $15: 12$ & 5714160 & Normal & OK & Normal $\left(23^{\circ} \mathrm{C}\right)$ & Normal (56\%) & OK & $\mathrm{OK}$ & 135 & -89.014444 & 41.367500 \\
\hline $8 / 28 / 09$ 15:15 & $15: 18$ & 5714160 & Normal & OK & Normal $\left(23^{\circ} \mathrm{C}\right)$ & Normal (56\%) & OK & OK & 135 & -89.058333 & 41.413333 \\
\hline $8 / 28 / 09$ 15:20 & $15: 23$ & 5714160 & Normal & OK & Normal $\left(23^{\circ} \mathrm{C}\right)$ & Normal (56\%) & OK & OK & 135 & -89.050833 & 41.460278 \\
\hline $8 / 28 / 09$ 15:25 & $15: 29$ & 5714160 & Normal & OK & Normal $\left(23^{\circ} \mathrm{C}\right)$ & Normal (56\%) & OK & OK & 135 & -89.058333 & 41.375833 \\
\hline $8 / 28 / 09$ 15:30 & $15: 35$ & 5714160 & Normal & OK & Normal $\left(23^{\circ} \mathrm{C}\right)$ & Normal (56\%) & OK & OK & 135 & -89.125833 & 41.368611 \\
\hline $8 / 28 / 09$ 15:35 & $15: 40$ & 5714160 & Normal & OK & Normal $\left(23^{\circ} \mathrm{C}\right)$ & Normal (56\%) & OK & OK & 135 & -89.125833 & 41.368611 \\
\hline $8 / 28 / 0915: 40$ & $15: 46$ & 5714160 & Normal & OK & Normal $\left(23^{\circ} \mathrm{C}\right)$ & Normal (56\%) & OK & OK & 135 & -89.125833 & 41.368611 \\
\hline $8 / 28 / 09$ 15:45 & $15: 51$ & 5714160 & Normal & OK & Normal $\left(23^{\circ} \mathrm{C}\right)$ & Normal (56\%) & OK & OK & 135 & -89.200000 & 41.364444 \\
\hline $8 / 28 / 0915: 50$ & $15: 57$ & 5714160 & Normal & OK & Normal $\left(23^{\circ} \mathrm{C}\right)$ & Normal $(56 \%)$ & OK & OK & 135 & -89.240556 & 41.371667 \\
\hline $8 / 28 / 09$ 16:00 & $16: 03$ & 5714160 & Normal & OK & Normal $\left(23^{\circ} \mathrm{C}\right)$ & Normal $(56 \%)$ & OK & OK & 135 & -89.458333 & 41.400833 \\
\hline $8 / 28 / 09$ 16:05 & $16: 13$ & 5714160 & Normal & OK & Normal $\left(23^{\circ} \mathrm{C}\right)$ & Normal $(56 \%)$ & OK & OK & 135 & -89.558333 & 41.393611 \\
\hline $8 / 28 / 09$ 16:11 & $16: 14$ & 5714160 & Normal & OK & Normal $\left(23^{\circ} \mathrm{C}\right)$ & Normal (56\%) & OK & OK & 135 & -89.558333 & 41.393611 \\
\hline $8 / 28 / 09$ 16:15 & $16: 19$ & 5714160 & Normal & OK & Normal $\left(23^{\circ} \mathrm{C}\right)$ & Normal (56\%) & OK & OK & 135 & -89.558333 & 41.393611 \\
\hline $8 / 28 / 0916: 20$ & $16: 25$ & 5714160 & Normal & OK & Normal $\left(23^{\circ} \mathrm{C}\right)$ & Normal (56\%) & OK & OK & 135 & -89.558333 & 41.393611 \\
\hline $8 / 28 / 0916: 25$ & $16: 30$ & 5714160 & Normal & OK & Normal $\left(23^{\circ} \mathrm{C}\right)$ & Normal $(56 \%)$ & OK & OK & 135 & -89.623889 & 41.394722 \\
\hline $8 / 28 / 0916: 30$ & $16: 36$ & 5714160 & Normal & OK & Normal $\left(23^{\circ} \mathrm{C}\right)$ & Normal (56\%) & OK & OK & 135 & -89.725833 & 41.387500 \\
\hline $8 / 28 / 09$ 16:35 & $16: 41$ & 5714160 & Normal & OK & Normal $\left(23^{\circ} \mathrm{C}\right)$ & Normal (56\%) & OK & $\mathrm{OK}$ & 135 & -89.834167 & 41.388333 \\
\hline
\end{tabular}




\begin{tabular}{|c|c|c|c|c|c|c|c|c|c|c|c|}
\hline Event Time & at QC Hub & Tag ID & Tag Status & Seal & Temperature & Humidity & Shock & Battery & RSSI & Longitude & Latitude \\
\hline $8 / 28 / 09$ 16:40 & $16: 47$ & 5714160 & Normal & OK & Normal $\left(23^{\circ} \mathrm{C}\right)$ & Normal $(56 \%)$ & OK & OK & 135 & -89.941667 & 41.416667 \\
\hline 8/28/09 16:45 & $16: 52$ & 5714160 & Normal & OK & Normal $\left(23^{\circ} \mathrm{C}\right)$ & Normal $(56 \%)$ & OK & OK & 135 & -90.050000 & 41.413333 \\
\hline $8 / 28 / 0916: 50$ & $16: 52$ & 5714160 & Normal & OK & Normal $\left(23^{\circ} \mathrm{C}\right)$ & Normal (56\%) & OK & OK & 135 & -90.157222 & 41.430000 \\
\hline 8/28/09 16:55 & $16: 58$ & 5714160 & Normal & OK & Normal $\left(23^{\circ} \mathrm{C}\right)$ & Normal (56\%) & OK & OK & 135 & -90.267500 & 41.440556 \\
\hline 8/28/09 17:00 & 17:09 & 5714160 & Normal & OK & Normal $\left(23^{\circ} \mathrm{C}\right)$ & Normal (56\%) & OK & OK & 135 & -90.338333 & 41.476944 \\
\hline 8/28/09 17:05 & 17:09 & 5714160 & Normal & OK & Normal $\left(23^{\circ} \mathrm{C}\right)$ & Normal $(56 \%)$ & OK & OK & 135 & -90.334167 & 41.486389 \\
\hline 8/28/09 17:11 & $17: 15$ & 5714160 & Normal & OK & Normal $\left(23^{\circ} \mathrm{C}\right)$ & Normal $(56 \%)$ & OK & OK & 135 & -90.334167 & 41.486389 \\
\hline $8 / 28 / 09$ 17:15 & $17: 20$ & 5714160 & Normal & OK & Normal $\left(23^{\circ} \mathrm{C}\right)$ & Normal $(56 \%)$ & OK & OK & 135 & -90.334167 & 41.486389 \\
\hline $8 / 28 / 09$ 17:20 & $17: 26$ & 5714160 & Normal & OK & Normal $\left(23^{\circ} \mathrm{C}\right)$ & Normal $(56 \%)$ & OK & OK & 135 & -90.334167 & 41.486389 \\
\hline $8 / 28 / 0917: 25$ & $17: 31$ & 5714160 & Normal & OK & Normal $\left(23^{\circ} \mathrm{C}\right)$ & Normal $(56 \%)$ & OK & OK & 135 & -90.340556 & 41.511389 \\
\hline $8 / 28 / 09$ 17:30 & $17: 37$ & 5714160 & Normal & OK & Normal $\left(23^{\circ} \mathrm{C}\right)$ & Normal (56\%) & OK & OK & 135 & -90.234167 & 41.562500 \\
\hline $8 / 28 / 0917: 35$ & $17: 42$ & 5714160 & Normal & OK & Normal $\left(23^{\circ} \mathrm{C}\right)$ & Normal (56\%) & OK & OK & 135 & -90.209167 & 41.581111 \\
\hline $8 / 28 / 0917: 40$ & $17: 42$ & 5714160 & Normal & OK & Normal $\left(23^{\circ} \mathrm{C}\right)$ & Normal (56\%) & OK & OK & 135 & -90.163333 & 41.642500 \\
\hline $8 / 28 / 0917: 45$ & $17: 48$ & 5714160 & Normal & OK & Normal $\left(23^{\circ} \mathrm{C}\right)$ & Normal (56\%) & OK & OK & 135 & -90.080000 & 41.690556 \\
\hline $8 / 28 / 0917: 50$ & $17: 53$ & 5714160 & Normal & OK & Normal $\left(23^{\circ} \mathrm{C}\right)$ & Normal (56\%) & OK & OK & 135 & -89.983333 & 41.718611 \\
\hline $8 / 28 / 0917: 55$ & $17: 59$ & 5714160 & Normal & OK & Normal $\left(25^{\circ} \mathrm{C}\right)$ & Normal $(56 \%)$ & OK & OK & 135 & -89.876944 & 41.744722 \\
\hline $8 / 28 / 0918: 00$ & 18:04 & 5714160 & Normal & OK & Normal $\left(25^{\circ} \mathrm{C}\right)$ & Normal (56\%) & OK & OK & 135 & -89.775000 & 41.757222 \\
\hline 8/28/09 18:05 & $18: 10$ & 5714160 & Normal & OK & Normal $\left(25^{\circ} \mathrm{C}\right)$ & Normal $(56 \%)$ & OK & OK & 135 & -89.689444 & 41.750833 \\
\hline $8 / 28 / 09$ 18:10 & $18: 15$ & 5714160 & Normal & OK & Normal $\left(25^{\circ} \mathrm{C}\right)$ & Normal (56\%) & OK & OK & 135 & -89.689444 & 41.762500 \\
\hline $8 / 28 / 0918: 15$ & $18: 21$ & 5714160 & Normal & OK & Normal $\left(25^{\circ} \mathrm{C}\right)$ & Normal (56\%) & OK & OK & 135 & -89.678056 & 41.750833 \\
\hline $8 / 28 / 0918: 20$ & $18: 26$ & 5714160 & Normal & OK & Normal $\left(25^{\circ} \mathrm{C}\right)$ & Normal (56\%) & OK & OK & 135 & -89.635278 & 41.759167 \\
\hline $8 / 28 / 09$ 18:25 & $18: 32$ & 5714160 & Normal & OK & Normal $\left(25^{\circ} \mathrm{C}\right)$ & Normal (56\%) & OK & OK & 135 & -89.532222 & 41.789444 \\
\hline $8 / 28 / 0918: 30$ & $18: 38$ & 5714160 & Normal & OK & Normal $\left(25^{\circ} \mathrm{C}\right)$ & Normal $(56 \%)$ & OK & OK & 135 & -89.432222 & 41.822778 \\
\hline $8 / 28 / 0918: 35$ & $18: 38$ & 5714160 & Normal & OK & Normal $\left(25^{\circ} \mathrm{C}\right)$ & Normal $(56 \%)$ & OK & OK & 135 & -89.336389 & 41.822778 \\
\hline $8 / 28 / 0918: 40$ & $18: 43$ & 5714160 & Normal & OK & Normal $\left(25^{\circ} \mathrm{C}\right)$ & Normal (56\%) & OK & OK & 135 & -89.231111 & 41.850833 \\
\hline $8 / 28 / 0918: 45$ & $18: 48$ & 5714160 & Normal & OK & Normal $\left(25^{\circ} \mathrm{C}\right)$ & Normal (55\%) & OK & OK & 135 & -89.125000 & 41.883333 \\
\hline $8 / 28 / 0918: 50$ & $18: 54$ & 5714160 & Normal & OK & Normal $\left(25^{\circ} \mathrm{C}\right)$ & Normal (55\%) & OK & OK & 135 & -89.025000 & 41.904167 \\
\hline $8 / 28 / 0918: 55$ & 19:00 & 5714160 & Normal & OK & Normal $\left(25^{\circ} \mathrm{C}\right)$ & Normal (55\%) & OK & OK & 135 & -88.913333 & 41.900000 \\
\hline $8 / 28 / 0919: 00$ & 19:05 & 5714160 & Normal & OK & Normal $\left(25^{\circ} \mathrm{C}\right)$ & Normal $(55 \%)$ & OK & OK & 135 & -88.814444 & 41.900000 \\
\hline $8 / 28 / 0919: 05$ & 19:11 & 5714160 & Normal & OK & Normal $\left(25^{\circ} \mathrm{C}\right)$ & Normal $(55 \%)$ & OK & OK & 135 & -88.739444 & 41.900000 \\
\hline 8/28/09 19:10 & $19: 16$ & 5714160 & Normal & OK & Normal $\left(25^{\circ} \mathrm{C}\right)$ & Normal (55\%) & OK & OK & 135 & -88.739444 & 41.900000 \\
\hline 8/28/09 19:15 & $19: 22$ & 5714160 & Normal & OK & Normal $\left(25^{\circ} \mathrm{C}\right)$ & Normal (55\%) & OK & OK & 135 & -88.737500 & 41.900000 \\
\hline $8 / 28 / 0919: 20$ & 19:27 & 5714160 & Normal & OK & Normal $\left(25^{\circ} \mathrm{C}\right)$ & Normal (55\%) & OK & OK & 135 & -88.737500 & 41.900000 \\
\hline $8 / 28 / 09$ 19:25 & $19: 33$ & 5714160 & Normal & OK & Normal $\left(25^{\circ} \mathrm{C}\right)$ & Normal (55\%) & OK & OK & 135 & -88.639444 & 41.894722 \\
\hline $8 / 28 / 09$ 19:30 & $19: 38$ & 5714160 & Normal & OK & Normal $\left(25^{\circ} \mathrm{C}\right)$ & Normal (55\%) & OK & OK & 135 & -88.538333 & 41.859167 \\
\hline $8 / 28 / 0919: 35$ & $19: 38$ & 5714160 & Normal & OK & Normal $\left(25^{\circ} \mathrm{C}\right)$ & Normal (55\%) & OK & OK & 135 & -88.448889 & 41.812500 \\
\hline $8 / 28 / 0919: 40$ & $19: 44$ & 5714160 & Normal & OK & Normal $\left(25^{\circ} \mathrm{C}\right)$ & Normal $(55 \%)$ & OK & OK & 135 & -88.347778 & 41.794722 \\
\hline $8 / 28 / 0919: 45$ & $19: 49$ & 5714160 & Normal & OK & Normal $\left(25^{\circ} \mathrm{C}\right)$ & Normal $(55 \%)$ & OK & OK & 135 & -88.261389 & 41.800000 \\
\hline $8 / 28 / 09$ 19:50 & $19: 55$ & 5714160 & Normal & OK & Normal $\left(25^{\circ} \mathrm{C}\right)$ & Normal (55\%) & OK & OK & 135 & -88.156111 & 41.806111 \\
\hline $8 / 28 / 09$ 19:55 & $20: 00$ & 5714160 & Normal & OK & Normal $\left(25^{\circ} \mathrm{C}\right)$ & Normal $(55 \%)$ & OK & OK & 135 & -88.062500 & 41.810278 \\
\hline $8 / 28 / 0920: 00$ & 20:06 & 5714160 & Normal & OK & Normal $\left(25^{\circ} \mathrm{C}\right)$ & Normal $(55 \%)$ & OK & OK & 135 & -88.054167 & 41.807222 \\
\hline $8 / 28 / 0920: 05$ & 20:11 & 5714160 & Normal & OK & Normal $\left(25^{\circ} \mathrm{C}\right)$ & Normal (55\%) & OK & OK & 135 & -88.054167 & 41.781111 \\
\hline 8/28/09 20:10 & $20: 22$ & 5714160 & Normal & OK & Normal $\left(25^{\circ} \mathrm{C}\right)$ & Normal (55\%) & $\mathrm{OK}$ & OK & 135 & -88.035278 & 41.732222 \\
\hline $8 / 28 / 0913: 40$ & $13: 44$ & 5714165 & Normal & $\mathrm{OK}$ & $\operatorname{Normal}\left(23^{\circ} \mathrm{C}\right)$ & Normal (56\%) & $\overline{\mathrm{OK}}$ & $\overline{O K}$ & 137 & -87.971667 & 41.732222 \\
\hline $8 / 28 / 0913: 45$ & $13: 49$ & 5714165 & Normal & OK & Normal $\left(23^{\circ} \mathrm{C}\right)$ & Normal (56\%) & OK & OK & 137 & -87.971667 & 41.732222 \\
\hline $8 / 28 / 0913: 50$ & $14: 00$ & 5714165 & Normal & OK & Normal $\left(23^{\circ} \mathrm{C}\right)$ & Normal (56\%) & OK & OK & 137 & -87.971667 & 41.732222 \\
\hline $8 / 28 / 0913: 55$ & $14: 00$ & 5714165 & Normal & OK & Normal $\left(23^{\circ} \mathrm{C}\right)$ & Normal (56\%) & OK & OK & 137 & -87.971667 & 41.732222 \\
\hline $8 / 28 / 09$ 14:00 & $14: 06$ & 5714165 & Normal & OK & Normal $\left(23^{\circ} \mathrm{C}\right)$ & Normal $(56 \%)$ & OK & OK & 137 & -88.068611 & 41.684167 \\
\hline $8 / 28 / 09$ 14:05 & $14: 11$ & 5714165 & Normal & OK & Normal $\left(23^{\circ} \mathrm{C}\right)$ & Normal (56\%) & OK & OK & 137 & -88.154167 & 41.640556 \\
\hline $8 / 28 / 0914: 10$ & $14: 17$ & 5714165 & Normal & OK & Normal $\left(23^{\circ} \mathrm{C}\right)$ & Normal $(56 \%)$ & OK & OK & 137 & -88.181111 & 41.568611 \\
\hline 8/28/09 14:15 & $14: 22$ & 5714165 & Normal & OK & Normal $\left(23^{\circ} \mathrm{C}\right)$ & Normal $(56 \%)$ & OK & OK & 137 & -88.197778 & 41.493611 \\
\hline $8 / 28 / 0914: 20$ & $14: 22$ & 5714165 & Normal & OK & Normal $\left(23^{\circ} \mathrm{C}\right)$ & Normal (56\%) & OK & OK & 137 & -88.280000 & 41.462500 \\
\hline $8 / 28 / 0914: 25$ & $14: 28$ & 5714165 & Normal & OK & Normal $\left(23^{\circ} \mathrm{C}\right)$ & Normal (56\%) & OK & OK & 137 & -88.371667 & 41.417500 \\
\hline $8 / 28 / 0914: 32$ & $14: 39$ & 5714165 & Normal & OK & Normal $\left(23^{\circ} \mathrm{C}\right)$ & Normal (56\%) & OK & OK & 137 & -88.422778 & 41.392500 \\
\hline $8 / 28 / 0914: 35$ & $14: 39$ & 5714165 & Normal & OK & Normal $\left(23^{\circ} \mathrm{C}\right)$ & Normal (56\%) & OK & OK & 137 & -88.422778 & 41.392500 \\
\hline $8 / 28 / 09$ 14:40 & $14: 44$ & 5714165 & Normal & OK & Normal $\left(23^{\circ} \mathrm{C}\right)$ & Normal (56\%) & OK & OK & 137 & -88.422778 & 41.392500 \\
\hline $8 / 28 / 0914: 45$ & $14: 50$ & 5714165 & Normal & OK & Normal $\left(23^{\circ} \mathrm{C}\right)$ & Normal $(56 \%)$ & OK & OK & 137 & -88.461389 & 41.378056 \\
\hline $8 / 28 / 0914: 50$ & $14: 56$ & 5714165 & Normal & OK & Normal $\left(23^{\circ} \mathrm{C}\right)$ & Normal $(56 \%)$ & OK & OK & 137 & -88.575000 & 41.376944 \\
\hline $8 / 28 / 0914: 55$ & 15:01 & 5714165 & Normal & OK & Normal $\left(23^{\circ} \mathrm{C}\right)$ & Normal (56\%) & OK & OK & 137 & -88.717500 & 41.375833 \\
\hline $8 / 28 / 0915: 00$ & 15:07 & 5714165 & Normal & OK & Normal $\left(23^{\circ} \mathrm{C}\right)$ & Normal (56\%) & OK & OK & 137 & -88.792500 & 41.376944 \\
\hline $8 / 28 / 0915: 05$ & $15: 12$ & 5714165 & Normal & OK & Normal $\left(23^{\circ} \mathrm{C}\right)$ & Normal (56\%) & OK & OK & 137 & -88.901944 & 41.368611 \\
\hline $8 / 28 / 09$ 15:10 & $15: 12$ & 5714165 & Normal & OK & Normal $\left(23^{\circ} \mathrm{C}\right)$ & Normal (56\%) & OK & OK & 137 & -89.014444 & 41.367500 \\
\hline $8 / 28 / 09$ 15:15 & $15: 18$ & 5714165 & Normal & OK & Normal $\left(23^{\circ} \mathrm{C}\right)$ & Normal (56\%) & OK & OK & 137 & -89.058333 & 41.413333 \\
\hline $8 / 28 / 0915: 20$ & $15: 23$ & 5714165 & Normal & OK & Normal $\left(23^{\circ} \mathrm{C}\right)$ & Normal (56\%) & OK & OK & 137 & -89.050833 & 41.460278 \\
\hline $8 / 28 / 09$ 15:25 & $15: 29$ & 5714165 & Normal & OK & Normal $\left(23^{\circ} \mathrm{C}\right)$ & Normal (56\%) & OK & OK & 137 & -89.058333 & 41.375833 \\
\hline $8 / 28 / 0915: 30$ & $15: 35$ & 5714165 & Normal & OK & Normal $\left(23^{\circ} \mathrm{C}\right)$ & Normal $(56 \%)$ & OK & OK & 137 & -89.125833 & 41.368611 \\
\hline 8/28/09 15:35 & $15: 40$ & 5714165 & Normal & OK & Normal $\left(23^{\circ} \mathrm{C}\right)$ & Normal (56\%) & OK & OK & 137 & -89.125833 & 41.368611 \\
\hline $8 / 28 / 0915: 40$ & $15: 46$ & 5714165 & Normal & OK & Normal $\left(23^{\circ} \mathrm{C}\right)$ & Normal (56\%) & OK & OK & 137 & -89.125833 & 41.368611 \\
\hline $8 / 28 / 0915: 45$ & $15: 51$ & 5714165 & Normal & OK & Normal $\left(23^{\circ} \mathrm{C}\right)$ & Normal (56\%) & OK & OK & 137 & -89.200000 & 41.364444 \\
\hline $8 / 28 / 0915: 50$ & $15: 57$ & 5714165 & Normal & OK & Normal $\left(23^{\circ} \mathrm{C}\right)$ & Normal (56\%) & OK & OK & 137 & -89.240556 & 41.371667 \\
\hline $8 / 28 / 0916: 00$ & $16: 03$ & 5714165 & Normal & OK & Normal $\left(23^{\circ} \mathrm{C}\right)$ & Normal $(56 \%)$ & OK & OK & 137 & -89.458333 & 41.400833 \\
\hline 8/28/09 16:05 & $16: 13$ & 5714165 & Normal & OK & Normal $\left(23^{\circ} \mathrm{C}\right)$ & Normal (56\%) & OK & OK & 137 & -89.558333 & 41.393611 \\
\hline $8 / 28 / 0916: 11$ & $16: 14$ & 5714165 & Normal & OK & Normal $\left(23^{\circ} \mathrm{C}\right)$ & Normal (56\%) & OK & OK & 137 & -89.558333 & 41.393611 \\
\hline 8/28/09 16:15 & $16: 19$ & 5714165 & Normal & OK & Normal $\left(23^{\circ} \mathrm{C}\right)$ & Normal $(56 \%)$ & OK & OK & 137 & -89.558333 & 41.393611 \\
\hline $8 / 28 / 0916: 20$ & $16: 25$ & 5714165 & Normal & OK & Normal $\left(23^{\circ} \mathrm{C}\right)$ & Normal (56\%) & OK & OK & 137 & -89.558333 & 41.393611 \\
\hline $8 / 28 / 0916: 25$ & $16: 30$ & 5714165 & Normal & OK & Normal $\left(23^{\circ} \mathrm{C}\right)$ & Normal (56\%) & OK & OK & 137 & -89.623889 & 41.394722 \\
\hline $8 / 28 / 0916: 30$ & $16: 36$ & 5714165 & Normal & OK & Normal $\left(23^{\circ} \mathrm{C}\right)$ & Normal (56\%) & OK & OK & 137 & -89.725833 & 41.387500 \\
\hline $8 / 28 / 0916: 35$ & $16: 41$ & 5714165 & Normal & OK & Normal $\left(23^{\circ} \mathrm{C}\right)$ & Normal (56\%) & OK & OK & 137 & -89.834167 & 41.388333 \\
\hline $8 / 28 / 0916: 40$ & $16: 47$ & 5714165 & Normal & OK & Normal $\left(23^{\circ} \mathrm{C}\right)$ & Normal (56\%) & OK & OK & 137 & -89.941667 & 41.416667 \\
\hline $8 / 28 / 0916: 45$ & $16: 52$ & 5714165 & Normal & OK & Normal $\left(23^{\circ} \mathrm{C}\right)$ & Normal (56\%) & OK & OK & 137 & -90.050000 & 41.413333 \\
\hline $8 / 28 / 0916: 50$ & $16: 52$ & 5714165 & Normal & OK & Normal $\left(23^{\circ} \mathrm{C}\right)$ & Normal (56\%) & OK & OK & 137 & -90.157222 & 41.430000 \\
\hline
\end{tabular}




\begin{tabular}{|c|c|c|c|c|c|c|c|c|c|c|c|}
\hline Event Time & at QC Hub & Tag ID & Tag Status & Seal & Temperature & Humidity & Shock & Battery & RSSI & Longitude & Latitude \\
\hline 8/28/09 16:55 & $16: 58$ & 5714165 & Normal & OK & Normal $\left(23^{\circ} \mathrm{C}\right)$ & Normal (56\%) & OK & OK & 137 & -90.267500 & 41.440556 \\
\hline 8/28/09 17:00 & $17: 09$ & 5714165 & Normal & OK & Normal $\left(23^{\circ} \mathrm{C}\right)$ & Normal (56\%) & OK & OK & 137 & -90.338333 & 41.476944 \\
\hline 8/28/09 17:05 & 17:09 & 5714165 & Normal & OK & Normal $\left(23^{\circ} \mathrm{C}\right)$ & Normal (56\%) & OK & OK & 137 & -90.334167 & 41.486389 \\
\hline 8/28/09 17:11 & $17: 15$ & 5714165 & Normal & OK & Normal $\left(23^{\circ} \mathrm{C}\right)$ & Normal (56\%) & OK & OK & 137 & -90.334167 & 41.486389 \\
\hline 8/28/09 17:15 & $17: 20$ & 5714165 & Normal & OK & Normal $\left(23^{\circ} \mathrm{C}\right)$ & Normal (56\%) & OK & $\mathrm{OK}$ & 137 & -90.334167 & 41.486389 \\
\hline 8/28/09 17:20 & $17: 26$ & 5714165 & Normal & OK & Normal $\left(23^{\circ} \mathrm{C}\right)$ & Normal (56\%) & OK & OK & 137 & -90.334167 & 41.486389 \\
\hline 8/28/09 17:25 & $17: 31$ & 5714165 & Normal & OK & Normal $\left(23^{\circ} \mathrm{C}\right)$ & Normal (56\%) & OK & $\mathrm{OK}$ & 137 & -90.340556 & 41.511389 \\
\hline 8/28/09 17:30 & $17: 37$ & 5714165 & Normal & OK & Normal $\left(23^{\circ} \mathrm{C}\right)$ & Normal (56\%) & $\mathrm{OK}$ & OK & 137 & -90.234167 & 41.562500 \\
\hline 8/28/09 17:35 & $17: 42$ & 5714165 & Normal & OK & Normal $\left(23^{\circ} \mathrm{C}\right)$ & Normal (56\%) & OK & OK & 137 & -90.209167 & 41.581111 \\
\hline 8/28/09 17:40 & $17: 42$ & 5714165 & Normal & OK & Normal $\left(23^{\circ} \mathrm{C}\right)$ & Normal (56\%) & OK & OK & 137 & -90.163333 & 41.642500 \\
\hline 8/28/09 17:45 & $17: 48$ & 5714165 & Normal & OK & Normal $\left(23^{\circ} \mathrm{C}\right)$ & Normal (56\%) & $\mathrm{OK}$ & $\mathrm{OK}$ & 137 & -90.080000 & 41.690556 \\
\hline 8/28/09 17:50 & $17: 53$ & 5714165 & Normal & OK & Normal $\left(23^{\circ} \mathrm{C}\right)$ & Normal (56\%) & $\mathrm{OK}$ & OK & 137 & -89.983333 & 41.718611 \\
\hline 8/28/09 17:55 & $17: 59$ & 5714165 & Normal & OK & Normal $\left(25^{\circ} \mathrm{C}\right)$ & Normal (56\%) & OK & OK & 137 & -89.876944 & 41.744722 \\
\hline $8 / 28 / 09$ 18:00 & $18: 04$ & 5714165 & Normal & OK & Normal $\left(25^{\circ} \mathrm{C}\right)$ & Normal (56\%) & OK & OK & 137 & -89.775000 & 41.757222 \\
\hline 8/28/09 18:05 & $18: 10$ & 5714165 & Normal & OK & Normal $\left(25^{\circ} \mathrm{C}\right)$ & Normal (56\%) & OK & OK & 137 & -89.689444 & 41.750833 \\
\hline 8/28/09 18:10 & $18: 15$ & 5714165 & Normal & OK & Normal $\left(25^{\circ} \mathrm{C}\right)$ & Normal $(56 \%)$ & OK & OK & 137 & -89.689444 & 41.762500 \\
\hline 8/28/09 18:15 & $18: 21$ & 5714165 & Normal & OK & Normal $\left(25^{\circ} \mathrm{C}\right)$ & Normal (56\%) & OK & OK & 137 & -89.678056 & 41.750833 \\
\hline 8/28/09 18:20 & $18: 26$ & 5714165 & Normal & OK & Normal $\left(25^{\circ} \mathrm{C}\right)$ & Normal (56\%) & OK & OK & 137 & -89.635278 & 41.759167 \\
\hline $8 / 28 / 09$ 18:25 & $18: 32$ & 5714165 & Normal & OK & Normal $\left(25^{\circ} \mathrm{C}\right)$ & Normal (56\%) & OK & OK & 137 & -89.532222 & 41.789444 \\
\hline 8/28/09 18:30 & $18: 38$ & 5714165 & Normal & OK & Normal $\left(25^{\circ} \mathrm{C}\right)$ & Normal (56\%) & OK & OK & 137 & -89.432222 & 41.822778 \\
\hline 8/28/09 18:35 & $18: 38$ & 5714165 & Normal & OK & Normal $\left(25^{\circ} \mathrm{C}\right)$ & Normal (56\%) & OK & OK & 137 & -89.336389 & 41.822778 \\
\hline $8 / 28 / 0918: 40$ & $18: 43$ & 5714165 & Normal & OK & Normal $\left(25^{\circ} \mathrm{C}\right)$ & Normal (56\%) & OK & OK & 137 & -89.231111 & 41.850833 \\
\hline $8 / 28 / 09$ 18:45 & $18: 48$ & 5714165 & Normal & OK & Normal $\left(25^{\circ} \mathrm{C}\right)$ & Normal (55\%) & OK & OK & 137 & -89.125000 & 41.883333 \\
\hline $8 / 28 / 09$ 18:50 & $18: 54$ & 5714165 & Normal & OK & Normal $\left(25^{\circ} \mathrm{C}\right)$ & Normal (55\%) & OK & OK & 137 & -89.025000 & 41.904167 \\
\hline $8 / 28 / 0918: 55$ & 19:00 & 5714165 & Normal & OK & Normal $\left(25^{\circ} \mathrm{C}\right)$ & Normal (55\%) & OK & OK & 137 & -88.913333 & 41.900000 \\
\hline 8/28/09 19:00 & $19: 05$ & 5714165 & Normal & OK & Normal $\left(25^{\circ} \mathrm{C}\right)$ & Normal (55\%) & OK & OK & 137 & -88.814444 & 41.900000 \\
\hline 8/28/09 19:05 & $19: 11$ & 5714165 & Normal & OK & Normal $\left(25^{\circ} \mathrm{C}\right)$ & Normal (55\%) & OK & OK & 137 & -88.739444 & 41.900000 \\
\hline 8/28/09 19:10 & $19: 16$ & 5714165 & Normal & OK & Normal $\left(25^{\circ} \mathrm{C}\right)$ & Normal (55\%) & OK & OK & 137 & -88.739444 & 41.900000 \\
\hline $8 / 28 / 09$ 19:15 & $19: 22$ & 5714165 & Normal & OK & Normal $\left(25^{\circ} \mathrm{C}\right)$ & Normal (55\%) & OK & OK & 137 & -88.737500 & 41.900000 \\
\hline $8 / 28 / 09$ 19:20 & $19: 27$ & 5714165 & Normal & OK & Normal $\left(25^{\circ} \mathrm{C}\right)$ & Normal (55\%) & OK & OK & 137 & -88.737500 & 41.900000 \\
\hline $8 / 28 / 09$ 19:25 & $19: 33$ & 5714165 & Normal & OK & Normal $\left(25^{\circ} \mathrm{C}\right)$ & Normal (55\%) & OK & OK & 137 & -88.639444 & 41.894722 \\
\hline $8 / 28 / 09$ 19:30 & $19: 38$ & 5714165 & Normal & OK & Normal $\left(25^{\circ} \mathrm{C}\right)$ & Normal (55\%) & OK & OK & 137 & -88.538333 & 41.859167 \\
\hline 8/28/09 19:35 & 19:38 & 5714165 & Normal & OK & Normal $\left(25^{\circ} \mathrm{C}\right)$ & Normal (55\%) & OK & OK & 137 & -88.448889 & 41.812500 \\
\hline $8 / 28 / 09$ 19:40 & $19: 44$ & 5714165 & Normal & OK & Normal $\left(25^{\circ} \mathrm{C}\right)$ & Normal (55\%) & OK & $\mathrm{OK}$ & 137 & -88.347778 & 41.794722 \\
\hline 8/28/09 19:45 & $19: 49$ & 5714165 & Normal & OK & Normal $\left(25^{\circ} \mathrm{C}\right)$ & Normal (55\%) & OK & OK & 137 & -88.261389 & 41.800000 \\
\hline $8 / 28 / 09$ 19:50 & 19:55 & 5714165 & Normal & OK & Normal $\left(25^{\circ} \mathrm{C}\right)$ & Normal (55\%) & OK & OK & 137 & -88.156111 & 41.806111 \\
\hline $8 / 28 / 09$ 19:55 & $20: 00$ & 5714165 & Normal & OK & Normal $\left(25^{\circ} \mathrm{C}\right)$ & Normal (55\%) & OK & OK & 137 & -88.062500 & 41.810278 \\
\hline 8/28/09 20:00 & 20:06 & 5714165 & Normal & OK & Normal $\left(25^{\circ} \mathrm{C}\right)$ & Normal (55\%) & OK & OK & 137 & -88.054167 & 41.807222 \\
\hline 8/28/09 20:05 & $20: 11$ & 5714165 & Normal & OK & Normal $\left(25^{\circ} \mathrm{C}\right)$ & Normal (55\%) & OK & OK & 137 & -88.054167 & 41.781111 \\
\hline $8 / 28 / 09$ 20:10 & $20: 22$ & 5714165 & Normal & OK & Normal $\left(25^{\circ} \mathrm{C}\right)$ & Normal $(55 \%)$ & OK & OK & 137 & -88.035278 & 41.732222 \\
\hline $8 / 28 / 09$ 13:40 & $13: 44$ & 5714170 & Normal & $\mathrm{OK}$ & Normal $\left(23^{\circ} \mathrm{C}\right)$ & Normal (56\%) & OK & OK & 140 & -87.971667 & 41.732222 \\
\hline 8/28/09 13:45 & $13: 49$ & 5714170 & Normal & OK & Normal $\left(23^{\circ} \mathrm{C}\right)$ & Normal (56\%) & OK & OK & 140 & -87.971667 & 41.732222 \\
\hline $8 / 28 / 09$ 13:50 & $14: 00$ & 5714170 & Normal & OK & Normal $\left(23^{\circ} \mathrm{C}\right)$ & Normal (56\%) & OK & OK & 140 & -87.971667 & 41.732222 \\
\hline $8 / 28 / 09$ 13:55 & $14: 00$ & 5714170 & Normal & OK & Normal $\left(23^{\circ} \mathrm{C}\right)$ & Normal (56\%) & OK & OK & 140 & -87.971667 & 41.732222 \\
\hline $8 / 28 / 09$ 14:00 & $14: 06$ & 5714170 & Normal & OK & Normal $\left(23^{\circ} \mathrm{C}\right)$ & Normal (56\%) & OK & OK & 140 & -88.068611 & 41.684167 \\
\hline 8/28/09 14:05 & $14: 11$ & 5714170 & Normal & OK & Normal $\left(23^{\circ} \mathrm{C}\right)$ & Normal (56\%) & OK & OK & 140 & -88.154167 & 41.640556 \\
\hline $8 / 28 / 09$ 14:10 & $14: 17$ & 5714170 & Normal & OK & Normal $\left(23^{\circ} \mathrm{C}\right)$ & Normal (56\%) & OK & OK & 140 & -88.181111 & 41.568611 \\
\hline 8/28/09 14:15 & $14: 22$ & 5714170 & Normal & OK & Normal $\left(23^{\circ} \mathrm{C}\right)$ & Normal (56\%) & OK & OK & 140 & -88.197778 & 41.493611 \\
\hline $8 / 28 / 09$ 14:20 & $14: 22$ & 5714170 & Normal & OK & Normal $\left(23^{\circ} \mathrm{C}\right)$ & Normal (56\%) & OK & OK & 140 & -88.280000 & 41.462500 \\
\hline $8 / 28 / 09$ 14:25 & $14: 28$ & 5714170 & Normal & OK & Normal $\left(23^{\circ} \mathrm{C}\right)$ & Normal (56\%) & OK & OK & 140 & -88.371667 & 41.417500 \\
\hline $8 / 28 / 09$ 14:32 & $14: 39$ & 5714170 & Normal & OK & Normal $\left(23^{\circ} \mathrm{C}\right)$ & Normal (56\%) & OK & OK & 140 & -88.422778 & 41.392500 \\
\hline $8 / 28 / 09$ 14:35 & $14: 39$ & 5714170 & Normal & OK & Normal $\left(23^{\circ} \mathrm{C}\right)$ & Normal (56\%) & OK & OK & 140 & -88.422778 & 41.392500 \\
\hline $8 / 28 / 09$ 14:40 & $14: 44$ & 5714170 & Normal & OK & Normal $\left(23^{\circ} \mathrm{C}\right)$ & Normal (56\%) & OK & OK & 140 & -88.422778 & 41.392500 \\
\hline $8 / 28 / 0914: 45$ & $14: 50$ & 5714170 & Normal & OK & Normal $\left(23^{\circ} \mathrm{C}\right)$ & Normal (56\%) & OK & OK & 140 & -88.461389 & 41.378056 \\
\hline $8 / 28 / 0914: 50$ & $14: 56$ & 5714170 & Normal & OK & Normal $\left(23^{\circ} \mathrm{C}\right)$ & Normal (56\%) & OK & OK & 140 & -88.575000 & 41.376944 \\
\hline $8 / 28 / 0914: 55$ & $15: 01$ & 5714170 & Normal & OK & Normal $\left(23^{\circ} \mathrm{C}\right)$ & Normal (56\%) & OK & OK & 140 & -88.717500 & 41.375833 \\
\hline $8 / 28 / 0915: 00$ & $15: 07$ & 5714170 & Normal & OK & Normal $\left(23^{\circ} \mathrm{C}\right)$ & Normal (56\%) & OK & OK & 140 & -88.792500 & 41.376944 \\
\hline $8 / 28 / 09$ 15:05 & $15: 12$ & 5714170 & Normal & OK & Normal $\left(23^{\circ} \mathrm{C}\right)$ & Normal (56\%) & OK & OK & 140 & -88.901944 & 41.368611 \\
\hline $8 / 28 / 09$ 15:10 & $15: 12$ & 5714170 & Normal & OK & Normal $\left(23^{\circ} \mathrm{C}\right)$ & Normal (56\%) & OK & OK & 140 & -89.014444 & 41.367500 \\
\hline $8 / 28 / 09$ 15:15 & $15: 18$ & 5714170 & Normal & OK & Normal $\left(23^{\circ} \mathrm{C}\right)$ & Normal (56\%) & OK & OK & 140 & -89.058333 & 41.413333 \\
\hline $8 / 28 / 09$ 15:20 & $15: 23$ & 5714170 & Normal & OK & Normal $\left(23^{\circ} \mathrm{C}\right)$ & Normal (56\%) & OK & OK & 140 & -89.050833 & 41.460278 \\
\hline $8 / 28 / 09$ 15:25 & $15: 29$ & 5714170 & Normal & OK & Normal $\left(23^{\circ} \mathrm{C}\right)$ & Normal (56\%) & OK & OK & 140 & -89.058333 & 41.375833 \\
\hline $8 / 28 / 09$ 15:30 & $15: 35$ & 5714170 & Normal & OK & Normal $\left(23^{\circ} \mathrm{C}\right)$ & Normal (56\%) & OK & OK & 140 & -89.125833 & 41.368611 \\
\hline $8 / 28 / 09$ 15:35 & $15: 40$ & 5714170 & Normal & OK & Normal $\left(23^{\circ} \mathrm{C}\right)$ & Normal (56\%) & OK & OK & 140 & -89.125833 & 41.368611 \\
\hline $8 / 28 / 0915: 40$ & $15: 46$ & 5714170 & Normal & oK & Normal $\left(23^{\circ} \mathrm{C}\right)$ & Normal (56\%) & OK & OK & 140 & -89.125833 & 41.368611 \\
\hline $8 / 28 / 09$ 15:45 & $15: 51$ & 5714170 & Normal & OK & Normal $\left(23^{\circ} \mathrm{C}\right)$ & Normal (56\%) & OK & OK & 140 & -89.200000 & 41.364444 \\
\hline $8 / 28 / 09$ 15:50 & $15: 57$ & 5714170 & Normal & OK & Normal $\left(23^{\circ} \mathrm{C}\right)$ & Normal (56\%) & OK & OK & 140 & -89.240556 & 41.371667 \\
\hline 8/28/09 16:00 & $16: 03$ & 5714170 & Normal & OK & Normal $\left(23^{\circ} \mathrm{C}\right)$ & Normal (56\%) & OK & OK & 140 & -89.458333 & 41.400833 \\
\hline 8/28/09 16:05 & $16: 13$ & 5714170 & Normal & OK & Normal $\left(23^{\circ} \mathrm{C}\right)$ & Normal (56\%) & OK & OK & 140 & -89.558333 & 41.393611 \\
\hline $8 / 28 / 09$ 16:11 & $16: 14$ & 5714170 & Normal & OK & Normal $\left(23^{\circ} \mathrm{C}\right)$ & Normal (56\%) & OK & OK & 140 & -89.558333 & 41.393611 \\
\hline 8/28/09 16:15 & $16: 19$ & 5714170 & Normal & OK & Normal $\left(23^{\circ} \mathrm{C}\right)$ & Normal (56\%) & OK & OK & 140 & -89.558333 & 41.393611 \\
\hline $8 / 28 / 09$ 16:20 & $16: 25$ & 5714170 & Normal & OK & Normal $\left(23^{\circ} \mathrm{C}\right)$ & Normal (56\%) & OK & OK & 140 & -89.558333 & 41.393611 \\
\hline $8 / 28 / 09$ 16:25 & $16: 30$ & 5714170 & Normal & OK & Normal $\left(23^{\circ} \mathrm{C}\right)$ & Normal (56\%) & OK & OK & 140 & -89.623889 & 41.394722 \\
\hline $8 / 28 / 09$ 16:30 & $16: 36$ & 5714170 & Normal & OK & Normal $\left(23^{\circ} \mathrm{C}\right)$ & Normal (56\%) & OK & OK & 140 & -89.725833 & 41.387500 \\
\hline $8 / 28 / 09$ 16:35 & $16: 41$ & 5714170 & Normal & OK & Normal $\left(23^{\circ} \mathrm{C}\right)$ & Normal (56\%) & OK & OK & 140 & -89.834167 & 41.388333 \\
\hline $8 / 28 / 09$ 16:40 & $16: 47$ & 5714170 & Normal & OK & Normal $\left(23^{\circ} \mathrm{C}\right)$ & Normal (56\%) & OK & OK & 140 & -89.941667 & 41.416667 \\
\hline 8/28/09 16:45 & $16: 52$ & 5714170 & Normal & oK & Normal $\left(23^{\circ} \mathrm{C}\right)$ & Normal (56\%) & OK & OK & 140 & -90.050000 & 41.413333 \\
\hline $8 / 28 / 09$ 16:50 & $16: 52$ & 5714170 & Normal & OK & Normal $\left(23^{\circ} \mathrm{C}\right)$ & Normal (56\%) & OK & OK & 140 & -90.157222 & 41.430000 \\
\hline $8 / 28 / 09$ 16:55 & $16: 58$ & 5714170 & Normal & OK & Normal $\left(23^{\circ} \mathrm{C}\right)$ & Normal (56\%) & OK & OK & 140 & -90.267500 & 41.440556 \\
\hline 8/28/09 17:00 & $17: 09$ & 5714170 & Normal & OK & Normal $\left(23^{\circ} \mathrm{C}\right)$ & Normal (56\%) & OK & OK & 140 & -90.338333 & 41.476944 \\
\hline 8/28/09 17:05 & $17: 09$ & 5714170 & Normal & OK & Normal $\left(23^{\circ} \mathrm{C}\right)$ & Normal (56\%) & OK & $\mathrm{OK}$ & 140 & -90.334167 & 41.486389 \\
\hline
\end{tabular}




\begin{tabular}{|c|c|c|c|c|c|c|c|c|c|c|c|}
\hline Event Time & at QC Hub & Tag ID & Tag Status & Seal & Temperature & Humidity & Shock & Battery & RSSI & Longitude & Latitude \\
\hline 8/28/09 17:11 & $17: 15$ & 5714170 & Normal & OK & Normal $\left(23^{\circ} \mathrm{C}\right)$ & Normal (56\%) & $\mathrm{OK}$ & OK & 140 & -90.334167 & 41.486389 \\
\hline 8/28/09 17:15 & $17: 20$ & 5714170 & Normal & OK & Normal $\left(23^{\circ} \mathrm{C}\right)$ & Normal (56\%) & $\mathrm{OK}$ & $\mathrm{OK}$ & 140 & -90.334167 & 41.486389 \\
\hline $8 / 28 / 09$ 17:20 & $17: 26$ & 5714170 & Normal & OK & Normal $\left(23^{\circ} \mathrm{C}\right)$ & Normal (56\%) & $\mathrm{OK}$ & OK & 140 & -90.334167 & 41.486389 \\
\hline 8/28/09 17:25 & $17: 31$ & 5714170 & Normal & OK & Normal $\left(23^{\circ} \mathrm{C}\right)$ & Normal (56\%) & $\mathrm{OK}$ & OK & 140 & -90.340556 & 41.511389 \\
\hline 8/28/09 17:30 & $17: 37$ & 5714170 & Normal & OK & Normal $\left(23^{\circ} \mathrm{C}\right)$ & Normal (56\%) & OK & OK & 140 & -90.234167 & 41.562500 \\
\hline $8 / 28 / 09$ 17:35 & $17: 42$ & 5714170 & Normal & OK & Normal $\left(23^{\circ} \mathrm{C}\right)$ & Normal (56\%) & OK & OK & 140 & -90.209167 & 41.581111 \\
\hline 8/28/09 17:40 & $17: 42$ & 5714170 & Normal & OK & Normal $\left(23^{\circ} \mathrm{C}\right)$ & Normal (56\%) & OK & OK & 140 & -90.163333 & 41.642500 \\
\hline $8 / 28 / 09$ 17:45 & $17: 48$ & 5714170 & Normal & OK & Normal $\left(23^{\circ} \mathrm{C}\right)$ & Normal (56\%) & $\mathrm{OK}$ & OK & 140 & -90.080000 & 41.690556 \\
\hline $8 / 28 / 09$ 17:50 & $17: 53$ & 5714170 & Normal & OK & Normal $\left(23^{\circ} \mathrm{C}\right)$ & Normal (56\%) & OK & OK & 140 & -89.983333 & 41.718611 \\
\hline $8 / 28 / 09$ 17:55 & $17: 59$ & 5714170 & Normal & OK & Normal $\left(25^{\circ} \mathrm{C}\right)$ & Normal (56\%) & OK & OK & 140 & -89.876944 & 41.744722 \\
\hline 8/28/09 18:00 & $18: 04$ & 5714170 & Normal & OK & Normal $\left(25^{\circ} \mathrm{C}\right)$ & Normal (56\%) & OK & OK & 140 & -89.775000 & 41.757222 \\
\hline 8/28/09 18:05 & $18: 10$ & 5714170 & Normal & $\mathrm{OK}$ & Normal $\left(25^{\circ} \mathrm{C}\right)$ & Normal (56\%) & OK & OK & 140 & -89.689444 & 41.750833 \\
\hline 8/28/09 18:10 & $18: 15$ & 5714170 & Normal & OK & Normal $\left(25^{\circ} \mathrm{C}\right)$ & Normal (56\%) & OK & OK & 140 & -89.689444 & 41.762500 \\
\hline 8/28/09 18:15 & $18: 21$ & 5714170 & Normal & OK & Normal $\left(25^{\circ} \mathrm{C}\right)$ & Normal (56\%) & OK & OK & 140 & -89.678056 & 41.750833 \\
\hline $8 / 28 / 09$ 18:20 & $18: 26$ & 5714170 & Normal & OK & Normal $\left(25^{\circ} \mathrm{C}\right)$ & Normal (56\%) & $\mathrm{OK}$ & OK & 140 & -89.635278 & 41.759167 \\
\hline 8/28/09 18:25 & $18: 32$ & 5714170 & Normal & OK & Normal $\left(25^{\circ} \mathrm{C}\right)$ & Normal (56\%) & $\mathrm{OK}$ & OK & 140 & -89.532222 & 41.789444 \\
\hline 8/28/09 18:30 & $18: 38$ & 5714170 & Normal & OK & Normal $\left(25^{\circ} \mathrm{C}\right)$ & Normal (56\%) & $\mathrm{OK}$ & OK & 140 & -89.432222 & 41.822778 \\
\hline 8/28/09 18:35 & $18: 38$ & 5714170 & Normal & OK & Normal $\left(25^{\circ} \mathrm{C}\right)$ & Normal (56\%) & $\mathrm{OK}$ & $\mathrm{OK}$ & 140 & -89.336389 & 41.822778 \\
\hline $8 / 28 / 0918: 40$ & $18: 43$ & 5714170 & Normal & OK & Normal $\left(25^{\circ} \mathrm{C}\right)$ & Normal $(56 \%)$ & OK & OK & 140 & -89.231111 & 41.850833 \\
\hline 8/28/09 18:45 & $18: 48$ & 5714170 & Normal & OK & Normal $\left(25^{\circ} \mathrm{C}\right)$ & Normal (55\%) & OK & OK & 140 & -89.125000 & 41.883333 \\
\hline $8 / 28 / 09$ 18:50 & $18: 54$ & 5714170 & Normal & OK & Normal $\left(25^{\circ} \mathrm{C}\right)$ & Normal (55\%) & OK & OK & 140 & -89.025000 & 41.904167 \\
\hline $8 / 28 / 09$ 18:55 & $19: 00$ & 5714170 & Normal & OK & Normal $\left(25^{\circ} \mathrm{C}\right)$ & Normal (55\%) & OK & OK & 140 & -88.913333 & 41.900000 \\
\hline $8 / 28 / 09$ 19:00 & 19:05 & 5714170 & Normal & OK & Normal $\left(25^{\circ} \mathrm{C}\right)$ & Normal (55\%) & OK & OK & 140 & -88.814444 & 41.900000 \\
\hline $8 / 28 / 09$ 19:05 & 19:11 & 5714170 & Normal & OK & Normal $\left(25^{\circ} \mathrm{C}\right)$ & Normal (55\%) & OK & OK & 140 & -88.739444 & 41.900000 \\
\hline 8/28/09 19:10 & 19:16 & 5714170 & Normal & OK & Normal $\left(25^{\circ} \mathrm{C}\right)$ & Normal (55\%) & OK & OK & 140 & -88.739444 & 41.900000 \\
\hline $8 / 28 / 09$ 19:15 & $19: 22$ & 5714170 & Normal & OK & Normal $\left(25^{\circ} \mathrm{C}\right)$ & Normal (55\%) & OK & OK & 140 & -88.737500 & 41.900000 \\
\hline $8 / 28 / 09$ 19:20 & $19: 27$ & 5714170 & Normal & OK & Normal $\left(25^{\circ} \mathrm{C}\right)$ & Normal (55\%) & OK & OK & 140 & -88.737500 & 41.900000 \\
\hline $8 / 28 / 09$ 19:25 & $19: 33$ & 5714170 & Normal & OK & Normal $\left(25^{\circ} \mathrm{C}\right)$ & Normal (55\%) & OK & OK & 140 & -88.639444 & 41.894722 \\
\hline $8 / 28 / 09$ 19:30 & $19: 38$ & 5714170 & Normal & OK & Normal $\left(25^{\circ} \mathrm{C}\right)$ & Normal (55\%) & OK & OK & 140 & -88.538333 & 41.859167 \\
\hline $8 / 28 / 09$ 19:35 & $19: 38$ & 5714170 & Normal & OK & Normal $\left(25^{\circ} \mathrm{C}\right)$ & Normal (55\%) & OK & OK & 140 & -88.448889 & 41.812500 \\
\hline $8 / 28 / 09$ 19:40 & $19: 44$ & 5714170 & Normal & OK & Normal $\left(25^{\circ} \mathrm{C}\right)$ & Normal $(55 \%)$ & OK & OK & 140 & -88.347778 & 41.794722 \\
\hline $8 / 28 / 09$ 19:45 & $19: 49$ & 5714170 & Normal & OK & Normal $\left(25^{\circ} \mathrm{C}\right)$ & Normal (55\%) & OK & OK & 140 & -88.261389 & 41.800000 \\
\hline $8 / 28 / 09$ 19:50 & $19: 55$ & 5714170 & Normal & OK & Normal $\left(25^{\circ} \mathrm{C}\right)$ & Normal (55\%) & OK & OK & 140 & -88.156111 & 41.806111 \\
\hline $8 / 28 / 09$ 19:55 & $20: 00$ & 5714170 & Normal & OK & Normal $\left(25^{\circ} \mathrm{C}\right)$ & Normal (55\%) & OK & OK & 140 & -88.062500 & 41.810278 \\
\hline $8 / 28 / 0920: 00$ & $20: 06$ & 5714170 & Normal & OK & Normal $\left(25^{\circ} \mathrm{C}\right)$ & Normal (55\%) & OK & OK & 140 & -88.054167 & 41.807222 \\
\hline $8 / 28 / 0920: 05$ & $20: 11$ & 5714170 & Normal & OK & Normal $\left(25^{\circ} \mathrm{C}\right)$ & Normal (55\%) & $\mathrm{OK}$ & OK & 140 & -88.054167 & 41.781111 \\
\hline $8 / 28 / 09$ 20:10 & $20: 22$ & 5714170 & Normal & OK & Normal $\left(25^{\circ} \mathrm{C}\right)$ & Normal $(55 \%)$ & OK & OK & 140 & -88.035278 & 41.732222 \\
\hline $8 / 28 / 0913: 40$ & $13: 44$ & 5714200 & Normal & $\mathrm{OK}$ & Normal $\left(23^{\circ} \mathrm{C}\right)$ & Normal (56\%) & $\overline{O K}$ & $\overline{O K}$ & 136 & -87.971667 & 41.732222 \\
\hline 8/28/09 13:45 & $13: 49$ & 5714200 & Normal & OK & Normal $\left(23^{\circ} \mathrm{C}\right)$ & Normal (56\%) & OK & OK & 136 & -87.971667 & 41.732222 \\
\hline $8 / 28 / 09$ 13:50 & $14: 00$ & 5714200 & Normal & OK & Normal $\left(23^{\circ} \mathrm{C}\right)$ & Normal (56\%) & OK & OK & 136 & -87.971667 & 41.732222 \\
\hline $8 / 28 / 09$ 13:55 & $14: 00$ & 5714200 & Normal & OK & Normal $\left(23^{\circ} \mathrm{C}\right)$ & Normal (56\%) & OK & OK & 136 & -87.971667 & 41.732222 \\
\hline $8 / 28 / 09$ 14:00 & $14: 06$ & 5714200 & Normal & OK & Normal $\left(23^{\circ} \mathrm{C}\right)$ & Normal (56\%) & OK & OK & 136 & -88.068611 & 41.684167 \\
\hline $8 / 28 / 09$ 14:05 & $14: 11$ & 5714200 & Normal & OK & Normal $\left(23^{\circ} \mathrm{C}\right)$ & Normal $(56 \%)$ & OK & OK & 136 & -88.154167 & 41.640556 \\
\hline $8 / 28 / 09$ 14:10 & $14: 17$ & 5714200 & Normal & OK & Normal $\left(23^{\circ} \mathrm{C}\right)$ & Normal $(56 \%)$ & OK & OK & 136 & -88.181111 & 41.568611 \\
\hline $8 / 28 / 09$ 14:15 & $14: 22$ & 5714200 & Normal & OK & Normal $\left(23^{\circ} \mathrm{C}\right)$ & Normal $(56 \%)$ & OK & OK & 136 & -88.197778 & 41.493611 \\
\hline $8 / 28 / 0914: 20$ & $14: 22$ & 5714200 & Normal & OK & Normal $\left(23^{\circ} \mathrm{C}\right)$ & Normal (56\%) & OK & OK & 136 & -88.280000 & 41.462500 \\
\hline $8 / 28 / 09$ 14:25 & $14: 28$ & 5714200 & Normal & OK & Normal $\left(23^{\circ} \mathrm{C}\right)$ & Normal (56\%) & OK & OK & 136 & -88.371667 & 41.417500 \\
\hline $8 / 28 / 09$ 14:32 & $14: 39$ & 5714200 & Normal & OK & Normal $\left(23^{\circ} \mathrm{C}\right)$ & Normal (56\%) & OK & OK & 136 & -88.422778 & 41.392500 \\
\hline $8 / 28 / 09$ 14:35 & $14: 39$ & 5714200 & Normal & OK & Normal $\left(23^{\circ} \mathrm{C}\right)$ & Normal (56\%) & OK & OK & 136 & -88.422778 & 41.392500 \\
\hline $8 / 28 / 09$ 14:40 & $14: 44$ & 5714200 & Normal & OK & Normal $\left(23^{\circ} \mathrm{C}\right)$ & Normal (56\%) & OK & OK & 136 & -88.422778 & 41.392500 \\
\hline $8 / 28 / 09$ 14:45 & $14: 50$ & 5714200 & Normal & OK & Normal $\left(23^{\circ} \mathrm{C}\right)$ & Normal (56\%) & OK & OK & 136 & -88.461389 & 41.378056 \\
\hline $8 / 28 / 0914: 50$ & $14: 56$ & 5714200 & Normal & OK & Normal $\left(23^{\circ} \mathrm{C}\right)$ & Normal $(56 \%)$ & OK & OK & 136 & -88.575000 & 41.376944 \\
\hline $8 / 28 / 09$ 14:55 & $15: 01$ & 5714200 & Normal & OK & Normal $\left(24^{\circ} \mathrm{C}\right)$ & Normal (55\%) & OK & OK & 136 & -88.717500 & 41.375833 \\
\hline $8 / 28 / 0915: 00$ & $15: 07$ & 5714200 & Normal & OK & Normal $\left(24^{\circ} \mathrm{C}\right)$ & Normal $(55 \%)$ & OK & OK & 136 & -88.792500 & 41.376944 \\
\hline $8 / 28 / 09$ 15:05 & $15: 12$ & 5714200 & Normal & OK & Normal $\left(24^{\circ} \mathrm{C}\right)$ & Normal $(55 \%)$ & OK & $\mathrm{OK}$ & 136 & -88.901944 & 41.368611 \\
\hline $8 / 28 / 09$ 15:10 & $15: 12$ & 5714200 & Normal & OK & Normal $\left(24^{\circ} \mathrm{C}\right)$ & Normal $(55 \%)$ & OK & OK & 136 & -89.014444 & 41.367500 \\
\hline $8 / 28 / 09$ 15:15 & $15: 18$ & 5714200 & Normal & OK & Normal $\left(24^{\circ} \mathrm{C}\right)$ & Normal (55\%) & OK & OK & 136 & -89.058333 & 41.413333 \\
\hline $8 / 28 / 09$ 15:20 & $15: 23$ & 5714200 & Normal & OK & Normal $\left(24^{\circ} \mathrm{C}\right)$ & Normal $(55 \%)$ & OK & OK & 136 & -89.050833 & 41.460278 \\
\hline $8 / 28 / 09$ 15:25 & $15: 29$ & 5714200 & Normal & OK & Normal $\left(24^{\circ} \mathrm{C}\right)$ & Normal (55\%) & OK & OK & 136 & -89.058333 & 41.375833 \\
\hline $8 / 28 / 09$ 15:30 & $15: 35$ & 5714200 & Normal & OK & Normal $\left(24^{\circ} \mathrm{C}\right)$ & Normal (55\%) & OK & OK & 136 & -89.125833 & 41.368611 \\
\hline $8 / 28 / 09$ 15:35 & $15: 40$ & 5714200 & Normal & OK & Normal $\left(24^{\circ} \mathrm{C}\right)$ & Normal (55\%) & OK & OK & 136 & -89.125833 & 41.368611 \\
\hline $8 / 28 / 0915: 40$ & $15: 46$ & 5714200 & Normal & OK & Normal $\left(24^{\circ} \mathrm{C}\right)$ & Normal (55\%) & OK & OK & 136 & -89.125833 & 41.368611 \\
\hline $8 / 28 / 09$ 15:45 & $15: 51$ & 5714200 & Normal & OK & Normal $\left(24^{\circ} \mathrm{C}\right)$ & Normal (55\%) & OK & OK & 136 & -89.200000 & 41.364444 \\
\hline $8 / 28 / 09$ 15:50 & $15: 57$ & 5714200 & Normal & OK & Normal $\left(24^{\circ} \mathrm{C}\right)$ & Normal (55\%) & OK & OK & 136 & -89.240556 & 41.371667 \\
\hline $8 / 28 / 0916: 00$ & $16: 03$ & 5714200 & Normal & OK & Normal $\left(24^{\circ} \mathrm{C}\right)$ & Normal (55\%) & $\mathrm{OK}$ & OK & 136 & -89.458333 & 41.400833 \\
\hline $8 / 28 / 09$ 16:05 & $16: 13$ & 5714200 & Normal & OK & Normal $\left(24^{\circ} \mathrm{C}\right)$ & Normal $(55 \%)$ & OK & OK & 136 & -89.558333 & 41.393611 \\
\hline $8 / 28 / 09$ 16:11 & $16: 14$ & 5714200 & Normal & OK & Normal $\left(24^{\circ} \mathrm{C}\right)$ & Normal (55\%) & OK & OK & 136 & -89.558333 & 41.393611 \\
\hline $8 / 28 / 09$ 16:15 & $16: 19$ & 5714200 & Normal & OK & Normal $\left(24^{\circ} \mathrm{C}\right)$ & Normal (55\%) & OK & OK & 136 & -89.558333 & 41.393611 \\
\hline $8 / 28 / 09$ 16:20 & $16: 25$ & 5714200 & Normal & OK & Normal $\left(24^{\circ} \mathrm{C}\right)$ & Normal (55\%) & OK & OK & 136 & -89.558333 & 41.393611 \\
\hline $8 / 28 / 09$ 16:25 & $16: 30$ & 5714200 & Normal & OK & Normal $\left(24^{\circ} \mathrm{C}\right)$ & Normal (55\%) & OK & OK & 136 & -89.623889 & 41.394722 \\
\hline $8 / 28 / 09$ 16:30 & $16: 36$ & 5714200 & Normal & OK & Normal $\left(24^{\circ} \mathrm{C}\right)$ & Normal (55\%) & OK & OK & 136 & -89.725833 & 41.387500 \\
\hline $8 / 28 / 09$ 16:35 & $16: 41$ & 5714200 & Normal & OK & Normal $\left(24^{\circ} \mathrm{C}\right)$ & Normal $(55 \%)$ & OK & OK & 136 & -89.834167 & 41.388333 \\
\hline $8 / 28 / 09$ 16:40 & $16: 47$ & 5714200 & Normal & OK & Normal $\left(24^{\circ} \mathrm{C}\right)$ & Normal $(55 \%)$ & OK & OK & 136 & -89.941667 & 41.416667 \\
\hline $8 / 28 / 09$ 16:45 & $16: 52$ & 5714200 & Normal & OK & Normal $\left(24^{\circ} \mathrm{C}\right)$ & Normal (55\%) & OK & OK & 136 & -90.050000 & 41.413333 \\
\hline $8 / 28 / 09$ 16:50 & $16: 52$ & 5714200 & Normal & OK & Normal $\left(24^{\circ} \mathrm{C}\right)$ & Normal (55\%) & OK & OK & 136 & -90.157222 & 41.430000 \\
\hline $8 / 28 / 09$ 16:55 & $16: 58$ & 5714200 & Normal & OK & Normal $\left(24^{\circ} \mathrm{C}\right)$ & Normal $(55 \%)$ & OK & OK & 136 & -90.267500 & 41.440556 \\
\hline $8 / 28 / 09$ 17:00 & 17:09 & 5714200 & Normal & OK & Normal $\left(24^{\circ} \mathrm{C}\right)$ & Normal (55\%) & OK & OK & 136 & -90.338333 & 41.476944 \\
\hline $8 / 28 / 09$ 17:05 & $17: 09$ & 5714200 & Normal & OK & Normal $\left(24^{\circ} \mathrm{C}\right)$ & Normal (55\%) & OK & OK & 136 & -90.334167 & 41.486389 \\
\hline $8 / 28 / 09$ 17:11 & $17: 15$ & 5714200 & Normal & OK & Normal $\left(24^{\circ} \mathrm{C}\right)$ & Normal (55\%) & OK & OK & 136 & -90.334167 & 41.486389 \\
\hline $8 / 28 / 09$ 17:15 & $17: 20$ & 5714200 & Normal & OK & Normal $\left(24^{\circ} \mathrm{C}\right)$ & Normal (55\%) & OK & OK & 136 & -90.334167 & 41.486389 \\
\hline $8 / 28 / 09$ 17:20 & $17: 26$ & 5714200 & Normal & OK & Normal $\left(24^{\circ} \mathrm{C}\right)$ & Normal (55\%) & OK & OK & 136 & -90.334167 & 41.486389 \\
\hline
\end{tabular}




\begin{tabular}{|c|c|c|c|c|c|c|c|c|c|c|c|}
\hline Event Time & at QC Hub & Tag ID & Tag Status & Seal & Temperature & Humidity & Shock & Battery & RSSI & Longitude & Latitude \\
\hline $8 / 28 / 09$ 17:25 & $17: 31$ & 5714200 & Normal & OK & Normal $\left(24^{\circ} \mathrm{C}\right)$ & Normal (55\%) & OK & OK & 136 & -90.340556 & 41.511389 \\
\hline $8 / 28 / 09$ 17:30 & $17: 37$ & 5714200 & Normal & $\mathrm{OK}$ & Normal $\left(24^{\circ} \mathrm{C}\right)$ & Normal $(55 \%)$ & OK & OK & 136 & -90.234167 & 41.562500 \\
\hline $8 / 28 / 0917: 35$ & $17: 42$ & 5714200 & Normal & OK & Normal $\left(24^{\circ} \mathrm{C}\right)$ & Normal (55\%) & OK & OK & 136 & -90.209167 & 41.581111 \\
\hline 8/28/09 17:40 & $17: 42$ & 5714200 & Normal & OK & Normal $\left(24^{\circ} \mathrm{C}\right)$ & Normal (55\%) & OK & OK & 136 & -90.163333 & 41.642500 \\
\hline $8 / 28 / 0917: 45$ & $17: 48$ & 5714200 & Normal & OK & Normal $\left(24^{\circ} \mathrm{C}\right)$ & Normal (55\%) & OK & OK & 136 & -90.080000 & 41.690556 \\
\hline $8 / 28 / 0917: 50$ & $17: 53$ & 5714200 & Normal & OK & Normal $\left(24^{\circ} \mathrm{C}\right)$ & Normal (55\%) & OK & OK & 136 & -89.983333 & 41.718611 \\
\hline $8 / 28 / 0917: 55$ & $17: 59$ & 5714200 & Normal & OK & Normal $\left(25^{\circ} \mathrm{C}\right)$ & Normal (55\%) & OK & OK & 136 & -89.876944 & 41.744722 \\
\hline $8 / 28 / 09$ 18:00 & $18: 04$ & 5714200 & Normal & OK & Normal $\left(25^{\circ} \mathrm{C}\right)$ & Normal (55\%) & OK & OK & 136 & -89.775000 & 41.757222 \\
\hline $8 / 28 / 09$ 18:05 & $18: 10$ & 5714200 & Normal & OK & Normal $\left(25^{\circ} \mathrm{C}\right)$ & Normal (55\%) & OK & OK & 136 & -89.689444 & 41.750833 \\
\hline $8 / 28 / 09$ 18:10 & $18: 15$ & 5714200 & Normal & OK & Normal $\left(25^{\circ} \mathrm{C}\right)$ & Normal (55\%) & OK & OK & 136 & -89.689444 & 41.762500 \\
\hline $8 / 28 / 09$ 18:15 & $18: 21$ & 5714200 & Normal & OK & Normal $\left(25^{\circ} \mathrm{C}\right)$ & Normal (55\%) & OK & OK & 136 & -89.678056 & 41.750833 \\
\hline $8 / 28 / 0918: 20$ & $18: 26$ & 5714200 & Normal & OK & Normal $\left(25^{\circ} \mathrm{C}\right)$ & Normal (55\%) & OK & OK & 136 & -89.635278 & 41.759167 \\
\hline $8 / 28 / 09$ 18:25 & $18: 32$ & 5714200 & Normal & OK & Normal $\left(25^{\circ} \mathrm{C}\right)$ & Normal $(55 \%)$ & OK & OK & 136 & -89.532222 & 41.789444 \\
\hline $8 / 28 / 09$ 18:30 & $18: 38$ & 5714200 & Normal & OK & Normal $\left(25^{\circ} \mathrm{C}\right)$ & Normal (55\%) & OK & OK & 136 & -89.432222 & 41.822778 \\
\hline $8 / 28 / 09$ 18:35 & $18: 38$ & 5714200 & Normal & OK & Normal $\left(25^{\circ} \mathrm{C}\right)$ & Normal (55\%) & OK & OK & 136 & -89.336389 & 41.822778 \\
\hline $8 / 28 / 0918: 40$ & $18: 43$ & 5714200 & Normal & OK & Normal $\left(25^{\circ} \mathrm{C}\right)$ & Normal (55\%) & OK & OK & 136 & -89.231111 & 41.850833 \\
\hline $8 / 28 / 09$ 18:45 & $18: 48$ & 5714200 & Normal & OK & Normal $\left(25^{\circ} \mathrm{C}\right)$ & Normal (54\%) & OK & OK & 136 & -89.125000 & 41.883333 \\
\hline $8 / 28 / 09$ 18:50 & $18: 54$ & 5714200 & Normal & OK & Normal $\left(25^{\circ} \mathrm{C}\right)$ & Normal $(54 \%)$ & OK & OK & 136 & -89.025000 & 41.904167 \\
\hline $8 / 28 / 09$ 18:55 & 19:00 & 5714200 & Normal & OK & Normal $\left(25^{\circ} \mathrm{C}\right)$ & Normal (54\%) & OK & OK & 136 & -88.913333 & 41.900000 \\
\hline 8/28/09 19:00 & 19:05 & 5714200 & Normal & OK & Normal $\left(25^{\circ} \mathrm{C}\right)$ & Normal (54\%) & OK & OK & 136 & -88.814444 & 41.900000 \\
\hline 8/28/09 19:05 & $19: 11$ & 5714200 & Normal & OK & Normal $\left(25^{\circ} \mathrm{C}\right)$ & Normal (54\%) & OK & OK & 136 & -88.739444 & 41.900000 \\
\hline $8 / 28 / 09$ 19:10 & $19: 16$ & 5714200 & Normal & OK & Normal $\left(25^{\circ} \mathrm{C}\right)$ & Normal (54\%) & OK & OK & 136 & -88.739444 & 41.900000 \\
\hline $8 / 28 / 09$ 19:15 & $19: 22$ & 5714200 & Normal & OK & Normal $\left(25^{\circ} \mathrm{C}\right)$ & Normal (54\%) & OK & OK & 136 & -88.737500 & 41.900000 \\
\hline $8 / 28 / 09$ 19:20 & 19:27 & 5714200 & Normal & OK & Normal $\left(25^{\circ} \mathrm{C}\right)$ & Normal (54\%) & OK & OK & 136 & -88.737500 & 41.900000 \\
\hline 8/28/09 19:25 & $19: 33$ & 5714200 & Normal & OK & Normal $\left(25^{\circ} \mathrm{C}\right)$ & Normal (54\%) & OK & OK & 136 & -88.639444 & 41.894722 \\
\hline 8/28/09 19:30 & $19: 38$ & 5714200 & Normal & OK & Normal $\left(25^{\circ} \mathrm{C}\right)$ & Normal (54\%) & OK & OK & 136 & -88.538333 & 41.859167 \\
\hline 8/28/09 19:35 & $19: 38$ & 5714200 & Normal & OK & Normal $\left(25^{\circ} \mathrm{C}\right)$ & Normal (54\%) & OK & OK & 136 & -88.448889 & 41.812500 \\
\hline $8 / 28 / 09$ 19:40 & $19: 44$ & 5714200 & Normal & OK & Normal $\left(25^{\circ} \mathrm{C}\right)$ & Normal (54\%) & OK & OK & 136 & -88.347778 & 41.794722 \\
\hline $8 / 28 / 0919: 45$ & $19: 49$ & 5714200 & Normal & OK & Normal $\left(25^{\circ} \mathrm{C}\right)$ & Normal $(54 \%)$ & OK & OK & 136 & -88.261389 & 41.800000 \\
\hline $8 / 28 / 0919: 50$ & 19:55 & 5714200 & Normal & OK & Normal $\left(25^{\circ} \mathrm{C}\right)$ & Normal (54\%) & OK & OK & 136 & -88.156111 & 41.806111 \\
\hline 8/28/09 19:55 & $20: 00$ & 5714200 & Normal & OK & Normal $\left(25^{\circ} \mathrm{C}\right)$ & Normal (54\%) & OK & OK & 136 & -88.062500 & 41.810278 \\
\hline $8 / 28 / 0920: 00$ & 20:06 & 5714200 & Normal & OK & Normal $\left(25^{\circ} \mathrm{C}\right)$ & Normal (54\%) & OK & OK & 136 & -88.054167 & 41.807222 \\
\hline 8/28/09 20:05 & $20: 11$ & 5714200 & Normal & OK & Normal $\left(25^{\circ} \mathrm{C}\right)$ & Normal (54\%) & OK & OK & 136 & -88.054167 & 41.781111 \\
\hline $8 / 28 / 09$ 20:10 & $20: 22$ & 5714200 & Normal & OK & Normal $\left(25^{\circ} \mathrm{C}\right)$ & Normal (54\%) & OK & OK & 136 & -88.035278 & 41.732222 \\
\hline $8 / 28 / 0913: 40$ & $13: 44$ & 5714205 & Normal & OK & Normal $\left(23^{\circ} \mathrm{C}\right)$ & Normal (56\%) & OK & $\overline{O K}$ & 139 & -87.971667 & 41.732222 \\
\hline $8 / 28 / 09$ 13:45 & $13: 49$ & 5714205 & Normal & OK & Normal $\left(23^{\circ} \mathrm{C}\right)$ & Normal (56\%) & OK & OK & 139 & -87.971667 & 41.732222 \\
\hline $8 / 28 / 0913: 50$ & $14: 00$ & 5714205 & Normal & OK & Normal $\left(23^{\circ} \mathrm{C}\right)$ & Normal (56\%) & OK & OK & 139 & -87.971667 & 41.732222 \\
\hline $8 / 28 / 09$ 13:55 & $14: 00$ & 5714205 & Normal & OK & Normal $\left(23^{\circ} \mathrm{C}\right)$ & Normal $(56 \%)$ & OK & OK & 139 & -87.971667 & 41.732222 \\
\hline $8 / 28 / 0914: 00$ & $14: 06$ & 5714205 & Normal & OK & Normal $\left(23^{\circ} \mathrm{C}\right)$ & Normal (56\%) & OK & OK & 139 & -88.068611 & 41.684167 \\
\hline $8 / 28 / 09$ 14:05 & $14: 11$ & 5714205 & Normal & OK & Normal $\left(23^{\circ} \mathrm{C}\right)$ & Normal (56\%) & OK & OK & 139 & -88.154167 & 41.640556 \\
\hline $8 / 28 / 0914: 10$ & $14: 17$ & 5714205 & Normal & OK & Normal $\left(23^{\circ} \mathrm{C}\right)$ & Normal (56\%) & OK & OK & 139 & -88.181111 & 41.568611 \\
\hline $8 / 28 / 09$ 14:15 & $14: 22$ & 5714205 & Normal & OK & Normal $\left(23^{\circ} \mathrm{C}\right)$ & Normal (56\%) & OK & OK & 139 & -88.197778 & 41.493611 \\
\hline $8 / 28 / 0914: 20$ & $14: 22$ & 5714205 & Normal & OK & Normal $\left(23^{\circ} \mathrm{C}\right)$ & Normal (56\%) & OK & OK & 139 & -88.280000 & 41.462500 \\
\hline $8 / 28 / 09$ 14:25 & $14: 28$ & 5714205 & Normal & OK & Normal $\left(23^{\circ} \mathrm{C}\right)$ & Normal (56\%) & OK & OK & 139 & -88.371667 & 41.417500 \\
\hline $8 / 28 / 09$ 14:32 & $14: 39$ & 5714205 & Normal & OK & Normal $\left(23^{\circ} \mathrm{C}\right)$ & Normal (56\%) & OK & OK & 139 & -88.422778 & 41.392500 \\
\hline $8 / 28 / 09$ 14:35 & $14: 39$ & 5714205 & Normal & OK & Normal $\left(23^{\circ} \mathrm{C}\right)$ & Normal $(56 \%)$ & OK & OK & 139 & -88.422778 & 41.392500 \\
\hline $8 / 28 / 0914: 40$ & $14: 44$ & 5714205 & Normal & OK & Normal $\left(23^{\circ} \mathrm{C}\right)$ & Normal (56\%) & OK & OK & 139 & -88.422778 & 41.392500 \\
\hline $8 / 28 / 0914: 45$ & $14: 50$ & 5714205 & Normal & OK & Normal $\left(23^{\circ} \mathrm{C}\right)$ & Normal (56\%) & OK & OK & 139 & -88.461389 & 41.378056 \\
\hline $8 / 28 / 0914: 50$ & $14: 56$ & 5714205 & Normal & OK & Normal $\left(23^{\circ} \mathrm{C}\right)$ & Normal $(56 \%)$ & OK & OK & 139 & -88.575000 & 41.376944 \\
\hline $8 / 28 / 09$ 14:55 & $15: 01$ & 5714205 & Normal & OK & Normal $\left(23^{\circ} \mathrm{C}\right)$ & Normal (55\%) & OK & OK & 139 & -88.717500 & 41.375833 \\
\hline $8 / 28 / 09$ 15:00 & 15:07 & 5714205 & Normal & OK & Normal $\left(23^{\circ} \mathrm{C}\right)$ & Normal (55\%) & OK & OK & 139 & -88.792500 & 41.376944 \\
\hline $8 / 28 / 09$ 15:05 & $15: 12$ & 5714205 & Normal & OK & Normal $\left(23^{\circ} \mathrm{C}\right)$ & Normal (55\%) & OK & OK & 139 & -88.901944 & 41.368611 \\
\hline $8 / 28 / 09$ 15:10 & $15: 12$ & 5714205 & Normal & OK & Normal $\left(23^{\circ} \mathrm{C}\right)$ & Normal (55\%) & OK & OK & 139 & -89.014444 & 41.367500 \\
\hline $8 / 28 / 09$ 15:15 & $15: 18$ & 5714205 & Normal & OK & Normal $\left(23^{\circ} \mathrm{C}\right)$ & Normal (55\%) & OK & OK & 139 & -89.058333 & 41.413333 \\
\hline $8 / 28 / 09$ 15:20 & $15: 23$ & 5714205 & Normal & OK & Normal $\left(23^{\circ} \mathrm{C}\right)$ & Normal (55\%) & OK & OK & 139 & -89.050833 & 41.460278 \\
\hline $8 / 28 / 09$ 15:25 & $15: 29$ & 5714205 & Normal & OK & Normal $\left(23^{\circ} \mathrm{C}\right)$ & Normal (55\%) & OK & OK & 139 & -89.058333 & 41.375833 \\
\hline $8 / 28 / 09$ 15:30 & $15: 35$ & 5714205 & Normal & OK & Normal $\left(23^{\circ} \mathrm{C}\right)$ & Normal (55\%) & OK & OK & 139 & -89.125833 & 41.368611 \\
\hline $8 / 28 / 09$ 15:35 & $15: 40$ & 5714205 & Normal & OK & Normal $\left(23^{\circ} \mathrm{C}\right)$ & Normal $(55 \%)$ & OK & OK & 139 & -89.125833 & 41.368611 \\
\hline $8 / 28 / 09$ 15:40 & $15: 46$ & 5714205 & Normal & OK & Normal $\left(23^{\circ} \mathrm{C}\right)$ & Normal (55\%) & OK & OK & 139 & -89.125833 & 41.368611 \\
\hline $8 / 28 / 09$ 15:45 & $15: 51$ & 5714205 & Normal & OK & Normal $\left(23^{\circ} \mathrm{C}\right)$ & Normal (55\%) & OK & OK & 139 & -89.200000 & 41.364444 \\
\hline $8 / 28 / 09$ 15:50 & $15: 57$ & 5714205 & Normal & OK & Normal $\left(23^{\circ} \mathrm{C}\right)$ & Normal (55\%) & OK & OK & 139 & -89.240556 & 41.371667 \\
\hline $8 / 28 / 0916: 00$ & $16: 03$ & 5714205 & Normal & OK & Normal $\left(23^{\circ} \mathrm{C}\right)$ & Normal (55\%) & OK & OK & 139 & -89.458333 & 41.400833 \\
\hline $8 / 28 / 0916: 05$ & $16: 13$ & 5714205 & Normal & OK & Normal $\left(23^{\circ} \mathrm{C}\right)$ & Normal (55\%) & OK & OK & 139 & -89.558333 & 41.393611 \\
\hline $8 / 28 / 09$ 16:11 & $16: 14$ & 5714205 & Normal & OK & Normal $\left(23^{\circ} \mathrm{C}\right)$ & Normal (55\%) & OK & OK & 139 & -89.558333 & 41.393611 \\
\hline $8 / 28 / 09$ 16:15 & $16: 19$ & 5714205 & Normal & OK & Normal $\left(23^{\circ} \mathrm{C}\right)$ & Normal (55\%) & OK & OK & 139 & -89.558333 & 41.393611 \\
\hline $8 / 28 / 09$ 16:20 & $16: 25$ & 5714205 & Normal & OK & Normal $\left(23^{\circ} \mathrm{C}\right)$ & Normal (55\%) & OK & OK & 139 & -89.558333 & 41.393611 \\
\hline $8 / 28 / 09$ 16:25 & $16: 30$ & 5714205 & Normal & OK & Normal $\left(23^{\circ} \mathrm{C}\right)$ & Normal $(55 \%)$ & OK & OK & 139 & -89.623889 & 41.394722 \\
\hline $8 / 28 / 0916: 30$ & $16: 36$ & 5714205 & Normal & OK & Normal $\left(23^{\circ} \mathrm{C}\right)$ & Normal (55\%) & OK & OK & 139 & -89.725833 & 41.387500 \\
\hline $8 / 28 / 0916: 35$ & $16: 41$ & 5714205 & Normal & OK & Normal $\left(23^{\circ} \mathrm{C}\right)$ & Normal (55\%) & OK & OK & 139 & -89.834167 & 41.388333 \\
\hline $8 / 28 / 0916: 40$ & $16: 47$ & 5714205 & Normal & OK & Normal $\left(23^{\circ} \mathrm{C}\right)$ & Normal (55\%) & OK & OK & 139 & -89.941667 & 41.416667 \\
\hline $8 / 28 / 09$ 16:45 & $16: 52$ & 5714205 & Normal & OK & Normal $\left(23^{\circ} \mathrm{C}\right)$ & Normal (55\%) & OK & OK & 139 & -90.050000 & 41.413333 \\
\hline $8 / 28 / 0916: 50$ & $16: 52$ & 5714205 & Normal & OK & Normal $\left(23^{\circ} \mathrm{C}\right)$ & Normal (55\%) & OK & OK & 139 & -90.157222 & 41.430000 \\
\hline $8 / 28 / 09$ 16:55 & $16: 58$ & 5714205 & Normal & OK & Normal $\left(23^{\circ} \mathrm{C}\right)$ & Normal (55\%) & OK & OK & 139 & -90.267500 & 41.440556 \\
\hline 8/28/09 17:00 & $17: 09$ & 5714205 & Normal & OK & Normal $\left(23^{\circ} \mathrm{C}\right)$ & Normal $(55 \%)$ & OK & OK & 139 & -90.338333 & 41.476944 \\
\hline $8 / 28 / 09$ 17:05 & $17: 09$ & 5714205 & Normal & OK & Normal $\left(23^{\circ} \mathrm{C}\right)$ & Normal (55\%) & OK & OK & 139 & -90.334167 & 41.486389 \\
\hline $8 / 28 / 0917: 11$ & $17: 15$ & 5714205 & Normal & OK & Normal $\left(23^{\circ} \mathrm{C}\right)$ & Normal (55\%) & OK & OK & 139 & -90.334167 & 41.486389 \\
\hline $8 / 28 / 09$ 17:15 & $17: 20$ & 5714205 & Normal & OK & Normal $\left(23^{\circ} \mathrm{C}\right)$ & Normal (55\%) & OK & OK & 139 & -90.334167 & 41.486389 \\
\hline $8 / 28 / 09$ 17:20 & $17: 26$ & 5714205 & Normal & OK & Normal $\left(23^{\circ} \mathrm{C}\right)$ & Normal (55\%) & OK & OK & 139 & -90.334167 & 41.486389 \\
\hline $8 / 28 / 09$ 17:25 & $17: 31$ & 5714205 & Normal & OK & Normal $\left(23^{\circ} \mathrm{C}\right)$ & Normal (55\%) & OK & OK & 139 & -90.340556 & 41.511389 \\
\hline $8 / 28 / 09$ 17:30 & $17: 37$ & 5714205 & Normal & OK & Normal $\left(23^{\circ} \mathrm{C}\right)$ & Normal (55\%) & OK & OK & 139 & -90.234167 & 41.562500 \\
\hline $8 / 28 / 09$ 17:35 & $17: 42$ & 5714205 & Normal & OK & Normal $\left(23^{\circ} \mathrm{C}\right)$ & Normal (55\%) & OK & OK & 139 & -90.209167 & 41.581111 \\
\hline
\end{tabular}




\begin{tabular}{|c|c|c|c|c|c|c|c|c|c|c|c|}
\hline Event Time & at QC Hub & Tag ID & Tag Status & Seal & Temperature & Humidity & Shock & Battery & RSSI & Longitude & Latitude \\
\hline $8 / 28 / 09$ 17:40 & $17: 42$ & 5714205 & Normal & OK & Normal $\left(23^{\circ} \mathrm{C}\right)$ & Normal (55\%) & $\mathrm{OK}$ & OK & 139 & -90.163333 & 41.642500 \\
\hline 8/28/09 17:45 & $17: 48$ & 5714205 & Normal & OK & Normal $\left(23^{\circ} \mathrm{C}\right)$ & Normal (55\%) & $\mathrm{OK}$ & $\mathrm{OK}$ & 139 & -90.080000 & 41.690556 \\
\hline $8 / 28 / 09$ 17:50 & $17: 53$ & 5714205 & Normal & OK & Normal $\left(23^{\circ} \mathrm{C}\right)$ & Normal (55\%) & OK & OK & 139 & -89.983333 & 41.718611 \\
\hline 8/28/09 17:55 & $17: 59$ & 5714205 & Normal & OK & Normal $\left(25^{\circ} \mathrm{C}\right)$ & Normal (55\%) & OK & $\mathrm{OK}$ & 139 & -89.876944 & 41.744722 \\
\hline 8/28/09 18:00 & $18: 04$ & 5714205 & Normal & OK & Normal $\left(25^{\circ} \mathrm{C}\right)$ & Normal (55\%) & $\mathrm{OK}$ & $\mathrm{OK}$ & 139 & -89.775000 & 41.757222 \\
\hline 8/28/09 18:05 & $18: 10$ & 5714205 & Normal & OK & Normal $\left(25^{\circ} \mathrm{C}\right)$ & Normal (55\%) & $\mathrm{OK}$ & OK & 139 & -89.689444 & 41.750833 \\
\hline 8/28/09 18:10 & $18: 15$ & 5714205 & Normal & OK & Normal $\left(25^{\circ} \mathrm{C}\right)$ & Normal (55\%) & $\mathrm{OK}$ & $\mathrm{OK}$ & 139 & -89.689444 & 41.762500 \\
\hline 8/28/09 18:15 & $18: 21$ & 5714205 & Normal & OK & Normal $\left(25^{\circ} \mathrm{C}\right)$ & Normal (55\%) & OK & $\mathrm{OK}$ & 139 & -89.678056 & 41.750833 \\
\hline $8 / 28 / 0918: 20$ & $18: 26$ & 5714205 & Normal & OK & Normal $\left(25^{\circ} \mathrm{C}\right)$ & Normal (55\%) & OK & $\mathrm{OK}$ & 139 & -89.635278 & 41.759167 \\
\hline $8 / 28 / 09$ 18:25 & $18: 32$ & 5714205 & Normal & OK & Normal $\left(25^{\circ} \mathrm{C}\right)$ & Normal (55\%) & OK & OK & 139 & -89.532222 & 41.789444 \\
\hline 8/28/09 18:30 & $18: 38$ & 5714205 & Normal & OK & Normal $\left(25^{\circ} \mathrm{C}\right)$ & Normal (55\%) & OK & OK & 139 & -89.432222 & 41.822778 \\
\hline 8/28/09 18:35 & $18: 38$ & 5714205 & Normal & OK & Normal $\left(25^{\circ} \mathrm{C}\right)$ & Normal (55\%) & $\mathrm{OK}$ & $\mathrm{OK}$ & 139 & -89.336389 & 41.822778 \\
\hline 8/28/09 18:40 & $18: 43$ & 5714205 & Normal & OK & Normal $\left(25^{\circ} \mathrm{C}\right)$ & Normal (55\%) & OK & OK & 139 & -89.231111 & 41.850833 \\
\hline $8 / 28 / 09$ 18:45 & $18: 48$ & 5714205 & Normal & OK & Normal $\left(25^{\circ} \mathrm{C}\right)$ & Normal (54\%) & $\mathrm{OK}$ & $\mathrm{OK}$ & 139 & -89.125000 & 41.883333 \\
\hline 8/28/09 18:50 & $18: 54$ & 5714205 & Normal & OK & Normal $\left(25^{\circ} \mathrm{C}\right)$ & Normal (54\%) & $\mathrm{OK}$ & $\mathrm{OK}$ & 139 & -89.025000 & 41.904167 \\
\hline 8/28/09 18:55 & 19:00 & 5714205 & Normal & OK & Normal $\left(25^{\circ} \mathrm{C}\right)$ & Normal (54\%) & $\mathrm{OK}$ & OK & 139 & -88.913333 & 41.900000 \\
\hline 8/28/09 19:00 & 19:05 & 5714205 & Normal & OK & Normal $\left(25^{\circ} \mathrm{C}\right)$ & Normal (54\%) & OK & OK & 139 & -88.814444 & 41.900000 \\
\hline 8/28/09 19:05 & 19:11 & 5714205 & Normal & OK & Normal $\left(25^{\circ} \mathrm{C}\right)$ & Normal (54\%) & OK & OK & 139 & -88.739444 & 41.900000 \\
\hline 8/28/09 19:10 & 19:16 & 5714205 & Normal & OK & Normal $\left(25^{\circ} \mathrm{C}\right)$ & Normal (54\%) & $\mathrm{OK}$ & OK & 139 & -88.739444 & 41.900000 \\
\hline 8/28/09 19:15 & $19: 22$ & 5714205 & Normal & OK & Normal $\left(25^{\circ} \mathrm{C}\right)$ & Normal (54\%) & OK & OK & 139 & -88.737500 & 41.900000 \\
\hline 8/28/09 19:20 & $19: 27$ & 5714205 & Normal & OK & Normal $\left(25^{\circ} \mathrm{C}\right)$ & Normal (54\%) & $\mathrm{OK}$ & OK & 139 & -88.737500 & 41.900000 \\
\hline $8 / 28 / 09$ 19:25 & $19: 33$ & 5714205 & Normal & OK & Normal $\left(25^{\circ} \mathrm{C}\right)$ & Normal (54\%) & OK & OK & 139 & -88.639444 & 41.894722 \\
\hline 8/28/09 19:30 & $19: 38$ & 5714205 & Normal & OK & Normal $\left(25^{\circ} \mathrm{C}\right)$ & Normal (54\%) & OK & OK & 139 & -88.538333 & 41.859167 \\
\hline $8 / 28 / 09$ 19:35 & $19: 38$ & 5714205 & Normal & OK & Normal $\left(25^{\circ} \mathrm{C}\right)$ & Normal $(54 \%)$ & OK & OK & 139 & -88.448889 & 41.812500 \\
\hline $8 / 28 / 09$ 19:40 & $19: 44$ & 5714205 & Normal & OK & Normal $\left(25^{\circ} \mathrm{C}\right)$ & Normal (54\%) & OK & OK & 139 & -88.347778 & 41.794722 \\
\hline $8 / 28 / 09$ 19:45 & $19: 49$ & 5714205 & Normal & OK & Normal $\left(25^{\circ} \mathrm{C}\right)$ & Normal (54\%) & OK & OK & 139 & -88.261389 & 41.800000 \\
\hline $8 / 28 / 09$ 19:50 & $19: 55$ & 5714205 & Normal & OK & Normal $\left(25^{\circ} \mathrm{C}\right)$ & Normal (54\%) & OK & OK & 139 & -88.156111 & 41.806111 \\
\hline 8/28/09 19:55 & $20: 00$ & 5714205 & Normal & OK & Normal $\left(25^{\circ} \mathrm{C}\right)$ & Normal (54\%) & OK & OK & 139 & -88.062500 & 41.810278 \\
\hline $8 / 28 / 09$ 20:00 & $20: 06$ & 5714205 & Normal & OK & Normal $\left(25^{\circ} \mathrm{C}\right)$ & Normal (54\%) & OK & OK & 139 & -88.054167 & 41.807222 \\
\hline $8 / 28 / 09$ 20:05 & $20: 11$ & 5714205 & Normal & OK & Normal $\left(25^{\circ} \mathrm{C}\right)$ & Normal (54\%) & OK & OK & 139 & -88.054167 & 41.781111 \\
\hline $8 / 28 / 09$ 20:10 & $20: 22$ & 5714205 & Normal & OK & Normal $\left(25^{\circ} \mathrm{C}\right)$ & Normal (54\%) & OK & OK & 139 & -88.035278 & 41.732222 \\
\hline $8 / 28 / 0913: 40$ & $13: 44$ & 5714210 & Normal & $\overline{O K}$ & Normal $\left(24^{\circ} \mathrm{C}\right)$ & Normal (54\%) & $\overline{O K}$ & $\overline{O K}$ & 139 & -87.971667 & 41.732222 \\
\hline $8 / 28 / 0913: 45$ & $13: 49$ & 5714210 & Normal & OK & Normal $\left(24^{\circ} \mathrm{C}\right)$ & Normal (54\%) & $\mathrm{OK}$ & OK & 139 & -87.971667 & 41.732222 \\
\hline 8/28/09 13:50 & $14: 00$ & 5714210 & Normal & OK & Normal $\left(24^{\circ} \mathrm{C}\right)$ & Normal (54\%) & OK & OK & 139 & -87.971667 & 41.732222 \\
\hline 8/28/09 13:55 & $14: 00$ & 5714210 & Normal & OK & Normal $\left(24^{\circ} \mathrm{C}\right)$ & Normal (54\%) & OK & OK & 139 & -87.971667 & 41.732222 \\
\hline $8 / 28 / 09$ 14:00 & $14: 06$ & 5714210 & Normal & OK & Normal $\left(24^{\circ} \mathrm{C}\right)$ & Normal (54\%) & $\mathrm{OK}$ & OK & 139 & -88.068611 & 41.684167 \\
\hline 8/28/09 14:05 & $14: 11$ & 5714210 & Normal & OK & Normal $\left(24^{\circ} \mathrm{C}\right)$ & Normal (54\%) & OK & $\mathrm{OK}$ & 139 & -88.154167 & 41.640556 \\
\hline $8 / 28 / 09$ 14:10 & $14: 17$ & 5714210 & Normal & OK & Normal $\left(24^{\circ} \mathrm{C}\right)$ & Normal (54\%) & $\mathrm{OK}$ & $\mathrm{OK}$ & 139 & -88.181111 & 41.568611 \\
\hline 8/28/09 14:15 & $14: 22$ & 5714210 & Normal & OK & Normal $\left(24^{\circ} \mathrm{C}\right)$ & Normal (54\%) & OK & OK & 139 & -88.197778 & 41.493611 \\
\hline $8 / 28 / 0914: 20$ & $14: 22$ & 5714210 & Normal & OK & Normal $\left(24^{\circ} \mathrm{C}\right)$ & Normal (54\%) & $\mathrm{OK}$ & OK & 139 & -88.280000 & 41.462500 \\
\hline $8 / 28 / 09$ 14:25 & $14: 28$ & 5714210 & Normal & OK & Normal $\left(24^{\circ} \mathrm{C}\right)$ & Normal (54\%) & OK & OK & 139 & -88.371667 & 41.417500 \\
\hline $8 / 28 / 09$ 14:32 & $14: 39$ & 5714210 & Normal & OK & Normal $\left(24^{\circ} \mathrm{C}\right)$ & Normal (54\%) & OK & OK & 139 & -88.422778 & 41.392500 \\
\hline $8 / 28 / 09$ 14:35 & $14: 39$ & 5714210 & Normal & OK & Normal $\left(24^{\circ} \mathrm{C}\right)$ & Normal (54\%) & OK & OK & 139 & -88.422778 & 41.392500 \\
\hline $8 / 28 / 09$ 14:40 & $14: 44$ & 5714210 & Normal & OK & Normal $\left(24^{\circ} \mathrm{C}\right)$ & Normal (54\%) & OK & OK & 139 & -88.422778 & 41.392500 \\
\hline 8/28/09 14:45 & $14: 50$ & 5714210 & Normal & OK & Normal $\left(24^{\circ} \mathrm{C}\right)$ & Normal (54\%) & OK & OK & 139 & -88.461389 & 41.378056 \\
\hline $8 / 28 / 09$ 14:50 & $14: 56$ & 5714210 & Normal & OK & Normal $\left(24^{\circ} \mathrm{C}\right)$ & Normal (54\%) & OK & OK & 139 & -88.575000 & 41.376944 \\
\hline $8 / 28 / 09$ 14:55 & $15: 01$ & 5714210 & Normal & OK & Normal $\left(24^{\circ} \mathrm{C}\right)$ & Normal (53\%) & OK & OK & 139 & -88.717500 & 41.375833 \\
\hline 8/28/09 15:00 & $15: 07$ & 5714210 & Normal & OK & Normal $\left(24^{\circ} \mathrm{C}\right)$ & Normal (53\%) & OK & OK & 139 & -88.792500 & 41.376944 \\
\hline $8 / 28 / 09$ 15:05 & $15: 12$ & 5714210 & Normal & OK & Normal $\left(24^{\circ} \mathrm{C}\right)$ & Normal (53\%) & OK & OK & 139 & -88.901944 & 41.368611 \\
\hline 8/28/09 15:10 & $15: 12$ & 5714210 & Normal & OK & Normal $\left(24^{\circ} \mathrm{C}\right)$ & Normal (53\%) & OK & OK & 139 & -89.014444 & 41.367500 \\
\hline 8/28/09 15:15 & $15: 18$ & 5714210 & Normal & OK & Normal $\left(24^{\circ} \mathrm{C}\right)$ & Normal (53\%) & OK & OK & 139 & -89.058333 & 41.413333 \\
\hline $8 / 28 / 09$ 15:20 & $15: 23$ & 5714210 & Normal & OK & Normal $\left(24^{\circ} \mathrm{C}\right)$ & Normal (53\%) & $\mathrm{OK}$ & OK & 139 & -89.050833 & 41.460278 \\
\hline $8 / 28 / 09$ 15:25 & $15: 29$ & 5714210 & Normal & OK & Normal $\left(24^{\circ} \mathrm{C}\right)$ & Normal $(53 \%)$ & OK & OK & 139 & -89.058333 & 41.375833 \\
\hline 8/28/09 15:30 & $15: 35$ & 5714210 & Normal & OK & Normal $\left(24^{\circ} \mathrm{C}\right)$ & Normal (53\%) & $\mathrm{OK}$ & $\mathrm{OK}$ & 139 & -89.125833 & 41.368611 \\
\hline 8/28/09 15:35 & $15: 40$ & 5714210 & Normal & OK & Normal $\left(24^{\circ} \mathrm{C}\right)$ & Normal (53\%) & $\mathrm{OK}$ & $\mathrm{OK}$ & 139 & -89.125833 & 41.368611 \\
\hline $8 / 28 / 09$ 15:40 & $15: 46$ & 5714210 & Normal & OK & Normal $\left(24^{\circ} \mathrm{C}\right)$ & Normal (53\%) & OK & OK & 139 & -89.125833 & 41.368611 \\
\hline 8/28/09 15:45 & $15: 51$ & 5714210 & Normal & OK & Normal $\left(24^{\circ} \mathrm{C}\right)$ & Normal (53\%) & OK & OK & 139 & -89.200000 & 41.364444 \\
\hline $8 / 28 / 09$ 15:50 & $15: 57$ & 5714210 & Normal & OK & Normal $\left(24^{\circ} \mathrm{C}\right)$ & Normal (53\%) & OK & OK & 139 & -89.240556 & 41.371667 \\
\hline $8 / 28 / 09$ 16:00 & $16: 03$ & 5714210 & Normal & OK & Normal $\left(24^{\circ} \mathrm{C}\right)$ & Normal (53\%) & OK & OK & 139 & -89.458333 & 41.400833 \\
\hline $8 / 28 / 09$ 16:05 & $16: 13$ & 5714210 & Normal & OK & Normal $\left(24^{\circ} \mathrm{C}\right)$ & Normal (53\%) & OK & OK & 139 & -89.558333 & 41.393611 \\
\hline $8 / 28 / 09$ 16:11 & $16: 14$ & 5714210 & Normal & OK & Normal $\left(24^{\circ} \mathrm{C}\right)$ & Normal (53\%) & OK & OK & 139 & -89.558333 & 41.393611 \\
\hline $8 / 28 / 09$ 16:15 & $16: 19$ & 5714210 & Normal & OK & Normal $\left(24^{\circ} \mathrm{C}\right)$ & Normal (53\%) & OK & OK & 139 & -89.558333 & 41.393611 \\
\hline $8 / 28 / 09$ 16:20 & $16: 25$ & 5714210 & Normal & OK & Normal $\left(24^{\circ} \mathrm{C}\right)$ & Normal (53\%) & OK & OK & 139 & -89.558333 & 41.393611 \\
\hline $8 / 28 / 09$ 16:25 & $16: 30$ & 5714210 & Normal & OK & Normal $\left(24^{\circ} \mathrm{C}\right)$ & Normal (53\%) & OK & OK & 139 & -89.623889 & 41.394722 \\
\hline $8 / 28 / 09$ 16:30 & $16: 36$ & 5714210 & Normal & OK & Normal $\left(24^{\circ} \mathrm{C}\right)$ & Normal (53\%) & OK & OK & 139 & -89.725833 & 41.387500 \\
\hline $8 / 28 / 09$ 16:35 & $16: 41$ & 5714210 & Normal & OK & Normal $\left(24^{\circ} \mathrm{C}\right)$ & Normal (53\%) & OK & OK & 139 & -89.834167 & 41.388333 \\
\hline $8 / 28 / 0916: 40$ & $16: 47$ & 5714210 & Normal & OK & Normal $\left(24^{\circ} \mathrm{C}\right)$ & Normal (53\%) & OK & OK & 139 & -89.941667 & 41.416667 \\
\hline $8 / 28 / 0916: 45$ & $16: 52$ & 5714210 & Normal & OK & Normal $\left(24^{\circ} \mathrm{C}\right)$ & Normal $(53 \%)$ & OK & OK & 139 & -90.050000 & 41.413333 \\
\hline $8 / 28 / 09$ 16:50 & $16: 52$ & 5714210 & Normal & OK & Normal $\left(24^{\circ} \mathrm{C}\right)$ & Normal (53\%) & OK & OK & 139 & -90.157222 & 41.430000 \\
\hline $8 / 28 / 09$ 16:55 & $16: 58$ & 5714210 & Normal & OK & Normal $\left(24^{\circ} \mathrm{C}\right)$ & Normal (53\%) & OK & OK & 139 & -90.267500 & 41.440556 \\
\hline $8 / 28 / 09$ 17:00 & $17: 09$ & 5714210 & Normal & OK & Normal $\left(24^{\circ} \mathrm{C}\right)$ & Normal (53\%) & OK & OK & 139 & -90.338333 & 41.476944 \\
\hline $8 / 28 / 09$ 17:05 & $17: 09$ & 5714210 & Normal & OK & Normal $\left(24^{\circ} \mathrm{C}\right)$ & Normal (53\%) & OK & OK & 139 & -90.334167 & 41.486389 \\
\hline $8 / 28 / 09$ 17:11 & $17: 15$ & 5714210 & Normal & OK & Normal $\left(24^{\circ} \mathrm{C}\right)$ & Normal $(53 \%)$ & OK & OK & 139 & -90.334167 & 41.486389 \\
\hline $8 / 28 / 09$ 17:15 & $17: 20$ & 5714210 & Normal & OK & Normal $\left(24^{\circ} \mathrm{C}\right)$ & Normal (53\%) & OK & OK & 139 & -90.334167 & 41.486389 \\
\hline $8 / 28 / 09$ 17:20 & $17: 26$ & 5714210 & Normal & OK & Normal $\left(24^{\circ} \mathrm{C}\right)$ & Normal (53\%) & OK & OK & 139 & -90.334167 & 41.486389 \\
\hline $8 / 28 / 09$ 17:25 & $17: 31$ & 5714210 & Normal & OK & Normal $\left(24^{\circ} \mathrm{C}\right)$ & Normal (53\%) & OK & OK & 139 & -90.340556 & 41.511389 \\
\hline $8 / 28 / 09$ 17:30 & $17: 37$ & 5714210 & Normal & OK & Normal $\left(24^{\circ} \mathrm{C}\right)$ & Normal (53\%) & OK & OK & 139 & -90.234167 & 41.562500 \\
\hline $8 / 28 / 09$ 17:35 & $17: 42$ & 5714210 & Normal & OK & Normal $\left(24^{\circ} \mathrm{C}\right)$ & Normal (53\%) & $\mathrm{OK}$ & OK & 139 & -90.209167 & 41.581111 \\
\hline $8 / 28 / 0917: 40$ & $17: 42$ & 5714210 & Normal & OK & Normal $\left(24^{\circ} \mathrm{C}\right)$ & Normal (53\%) & OK & OK & 139 & -90.163333 & 41.642500 \\
\hline $8 / 28 / 0917: 45$ & $17: 48$ & 5714210 & Normal & OK & Normal $\left(24^{\circ} \mathrm{C}\right)$ & Normal (53\%) & OK & $\mathrm{OK}$ & 139 & -90.080000 & 41.690556 \\
\hline $8 / 28 / 0917: 50$ & $17: 53$ & 5714210 & Normal & OK & Normal $\left(24^{\circ} \mathrm{C}\right)$ & Normal (53\%) & $\mathrm{OK}$ & OK & 139 & -89.983333 & 41.718611 \\
\hline
\end{tabular}




\begin{tabular}{|c|c|c|c|c|c|c|c|c|c|c|c|}
\hline Event Time & at QC Hub & Tag ID & Tag Status & Seal & Temperature & Humidity & Shock & Battery & RSSI & Longitude & Latitude \\
\hline $8 / 28 / 09$ 17:55 & $17: 59$ & 5714210 & Normal & OK & Normal $\left(25^{\circ} \mathrm{C}\right)$ & Normal (54\%) & OK & OK & 139 & -89.876944 & 41.744722 \\
\hline 8/28/09 18:00 & $18: 04$ & 5714210 & Normal & OK & Normal $\left(25^{\circ} \mathrm{C}\right)$ & Normal (54\%) & $\mathrm{OK}$ & OK & 139 & -89.775000 & 41.757222 \\
\hline 8/28/09 18:05 & $18: 10$ & 5714210 & Normal & OK & Normal $\left(25^{\circ} \mathrm{C}\right)$ & Normal (54\%) & OK & OK & 139 & -89.689444 & 41.750833 \\
\hline 8/28/09 18:10 & $18: 15$ & 5714210 & Normal & OK & Normal $\left(25^{\circ} \mathrm{C}\right)$ & Normal (54\%) & OK & OK & 139 & -89.689444 & 41.762500 \\
\hline 8/28/09 18:15 & $18: 21$ & 5714210 & Normal & OK & Normal $\left(25^{\circ} \mathrm{C}\right)$ & Normal (54\%) & OK & OK & 139 & -89.678056 & 41.750833 \\
\hline $8 / 28 / 09$ 18:20 & $18: 26$ & 5714210 & Normal & OK & Normal $\left(25^{\circ} \mathrm{C}\right)$ & Normal (54\%) & OK & OK & 139 & -89.635278 & 41.759167 \\
\hline $8 / 28 / 09$ 18:25 & $18: 32$ & 5714210 & Normal & OK & Normal $\left(25^{\circ} \mathrm{C}\right)$ & Normal (54\%) & OK & OK & 139 & -89.532222 & 41.789444 \\
\hline $8 / 28 / 09$ 18:30 & $18: 38$ & 5714210 & Normal & OK & Normal $\left(25^{\circ} \mathrm{C}\right)$ & Normal (54\%) & OK & OK & 139 & -89.432222 & 41.822778 \\
\hline 8/28/09 18:35 & $18: 38$ & 5714210 & Normal & OK & Normal $\left(25^{\circ} \mathrm{C}\right)$ & Normal (54\%) & OK & OK & 139 & -89.336389 & 41.822778 \\
\hline $8 / 28 / 0918: 40$ & $18: 43$ & 5714210 & Normal & OK & Normal $\left(25^{\circ} \mathrm{C}\right)$ & Normal (54\%) & OK & OK & 139 & -89.231111 & 41.850833 \\
\hline 8/28/09 18:45 & $18: 48$ & 5714210 & Normal & OK & Normal $\left(25^{\circ} \mathrm{C}\right)$ & Normal (54\%) & OK & OK & 139 & -89.125000 & 41.883333 \\
\hline 8/28/09 18:50 & $18: 54$ & 5714210 & Normal & OK & Normal $\left(25^{\circ} \mathrm{C}\right)$ & Normal (54\%) & OK & OK & 139 & -89.025000 & 41.904167 \\
\hline 8/28/09 18:55 & 19:00 & 5714210 & Normal & OK & Normal $\left(25^{\circ} \mathrm{C}\right)$ & Normal (54\%) & $\mathrm{OK}$ & OK & 139 & -88.913333 & 41.900000 \\
\hline 8/28/09 19:00 & 19:05 & 5714210 & Normal & OK & Normal $\left(25^{\circ} \mathrm{C}\right)$ & Normal (54\%) & OK & OK & 139 & -88.814444 & 41.900000 \\
\hline 8/28/09 19:05 & 19:11 & 5714210 & Normal & OK & Normal $\left(24^{\circ} \mathrm{C}\right)$ & Normal (54\%) & OK & OK & 139 & -88.739444 & 41.900000 \\
\hline 8/28/09 19:07 & 19:11 & 5714210 & Alarm & Open & Normal $\left(24^{\circ} \mathrm{C}\right)$ & Normal (54\%) & OK & OK & 139 & -88.739444 & 41.900000 \\
\hline 8/28/09 19:10 & $19: 16$ & 5714210 & Normal & OK & Normal $\left(24^{\circ} \mathrm{C}\right)$ & Normal (55\%) & OK & OK & 139 & -88.739444 & 41.900000 \\
\hline 8/28/09 19:15 & $19: 22$ & 5714210 & Normal & OK & Normal $\left(24^{\circ} \mathrm{C}\right)$ & Normal (55\%) & OK & OK & 139 & -88.737500 & 41.900000 \\
\hline $8 / 28 / 09$ 19:20 & $19: 27$ & 5714210 & Normal & OK & Normal $\left(24^{\circ} \mathrm{C}\right)$ & Normal (55\%) & OK & OK & 139 & -88.737500 & 41.900000 \\
\hline 8/28/09 19:25 & $19: 33$ & 5714210 & Normal & OK & Normal $\left(24^{\circ} \mathrm{C}\right)$ & Normal (55\%) & OK & OK & 139 & -88.639444 & 41.894722 \\
\hline 8/28/09 19:30 & $19: 38$ & 5714210 & Normal & OK & Normal $\left(24^{\circ} \mathrm{C}\right)$ & Normal (55\%) & OK & OK & 139 & -88.538333 & 41.859167 \\
\hline 8/28/09 19:35 & $19: 38$ & 5714210 & Normal & OK & Normal $\left(24^{\circ} \mathrm{C}\right)$ & Normal (55\%) & OK & OK & 139 & -88.448889 & 41.812500 \\
\hline $8 / 28 / 09$ 19:40 & $19: 44$ & 5714210 & Normal & OK & Normal $\left(24^{\circ} \mathrm{C}\right)$ & Normal (55\%) & OK & OK & 139 & -88.347778 & 41.794722 \\
\hline $8 / 28 / 09$ 19:45 & $19: 49$ & 5714210 & Normal & OK & Normal $\left(24^{\circ} \mathrm{C}\right)$ & Normal (55\%) & OK & OK & 139 & -88.261389 & 41.800000 \\
\hline 8/28/09 19:50 & $19: 55$ & 5714210 & Normal & OK & Normal $\left(24^{\circ} \mathrm{C}\right)$ & Normal (55\%) & OK & OK & 139 & -88.156111 & 41.806111 \\
\hline $8 / 28 / 09$ 19:55 & $20: 00$ & 5714210 & Normal & OK & Normal $\left(24^{\circ} \mathrm{C}\right)$ & Normal (55\%) & OK & OK & 139 & -88.062500 & 41.810278 \\
\hline $8 / 28 / 09$ 20:00 & $20: 06$ & 5714210 & Normal & OK & Normal $\left(24^{\circ} \mathrm{C}\right)$ & Normal (55\%) & OK & OK & 139 & -88.054167 & 41.807222 \\
\hline 8/28/09 20:05 & $20: 11$ & 5714210 & Normal & OK & Normal $\left(26^{\circ} \mathrm{C}\right)$ & Normal (54\%) & OK & OK & 139 & -88.054167 & 41.781111 \\
\hline $8 / 28 / 09$ 20:10 & $20: 22$ & 5714210 & Normal & OK & Normal $\left(26^{\circ} \mathrm{C}\right)$ & Normal (54\%) & OK & OK & 139 & -88.035278 & 41.732222 \\
\hline $8 / 28 / 0913: 40$ & $13: 44$ & 5714215 & Normal & OK & Normal $\left(24^{\circ} \mathrm{C}\right)$ & Normal (54\%) & $\mathrm{OK}$ & $\mathrm{OK}$ & 128 & -87.971667 & 41.732222 \\
\hline $8 / 28 / 09$ 13:45 & $13: 49$ & 5714215 & Normal & OK & Normal $\left(24^{\circ} \mathrm{C}\right)$ & Normal (54\%) & OK & OK & 128 & -87.971667 & 41.732222 \\
\hline 8/28/09 13:50 & $14: 00$ & 5714215 & Normal & OK & Normal $\left(24^{\circ} \mathrm{C}\right)$ & Normal (54\%) & OK & OK & 128 & -87.971667 & 41.732222 \\
\hline 8/28/09 13:55 & $14: 00$ & 5714215 & Normal & OK & Normal $\left(24^{\circ} \mathrm{C}\right)$ & Normal (54\%) & OK & OK & 128 & -87.971667 & 41.732222 \\
\hline $8 / 28 / 09$ 14:00 & $14: 06$ & 5714215 & Normal & OK & Normal $\left(24^{\circ} \mathrm{C}\right)$ & Normal (54\%) & OK & OK & 128 & -88.068611 & 41.684167 \\
\hline $8 / 28 / 09$ 14:05 & $14: 11$ & 5714215 & Normal & OK & Normal $\left(24^{\circ} \mathrm{C}\right)$ & Normal (54\%) & OK & OK & 128 & -88.154167 & 41.640556 \\
\hline $8 / 28 / 09$ 14:10 & $14: 17$ & 5714215 & Normal & OK & Normal $\left(24^{\circ} \mathrm{C}\right)$ & Normal (54\%) & OK & OK & 128 & -88.181111 & 41.568611 \\
\hline $8 / 28 / 09$ 14:15 & $14: 22$ & 5714215 & Normal & OK & Normal $\left(24^{\circ} \mathrm{C}\right)$ & Normal (54\%) & OK & OK & 128 & -88.197778 & 41.493611 \\
\hline $8 / 28 / 09$ 14:20 & $14: 22$ & 5714215 & Normal & OK & Normal $\left(24^{\circ} \mathrm{C}\right)$ & Normal (54\%) & OK & OK & 128 & -88.280000 & 41.462500 \\
\hline $8 / 28 / 09$ 14:25 & $14: 28$ & 5714215 & Normal & OK & Normal $\left(24^{\circ} \mathrm{C}\right)$ & Normal (54\%) & OK & OK & 128 & -88.371667 & 41.417500 \\
\hline $8 / 28 / 09$ 14:32 & $14: 39$ & 5714215 & Normal & OK & Normal $\left(24^{\circ} \mathrm{C}\right)$ & Normal (54\%) & OK & OK & 128 & -88.422778 & 41.392500 \\
\hline 8/28/09 14:35 & $14: 39$ & 5714215 & Normal & OK & Normal $\left(24^{\circ} \mathrm{C}\right)$ & Normal (54\%) & OK & OK & 128 & -88.422778 & 41.392500 \\
\hline $8 / 28 / 09$ 14:40 & $14: 44$ & 5714215 & Normal & OK & Normal $\left(24^{\circ} \mathrm{C}\right)$ & Normal (54\%) & OK & OK & 128 & -88.422778 & 41.392500 \\
\hline 8/28/09 14:45 & $14: 50$ & 5714215 & Normal & OK & Normal $\left(24^{\circ} \mathrm{C}\right)$ & Normal (54\%) & OK & OK & 128 & -88.461389 & 41.378056 \\
\hline $8 / 28 / 09$ 14:50 & $14: 56$ & 5714215 & Normal & OK & Normal $\left(24^{\circ} \mathrm{C}\right)$ & Normal (54\%) & OK & OK & 128 & -88.575000 & 41.376944 \\
\hline 8/28/09 14:55 & 15:01 & 5714215 & Normal & OK & Normal $\left(24^{\circ} \mathrm{C}\right)$ & Normal (54\%) & OK & OK & 128 & -88.717500 & 41.375833 \\
\hline 8/28/09 15:00 & 15:07 & 5714215 & Normal & OK & Normal $\left(24^{\circ} \mathrm{C}\right)$ & Normal (54\%) & OK & OK & 128 & -88.792500 & 41.376944 \\
\hline 8/28/09 15:05 & $15: 12$ & 5714215 & Normal & OK & Normal $\left(24^{\circ} \mathrm{C}\right)$ & Normal (54\%) & OK & OK & 128 & -88.901944 & 41.368611 \\
\hline $8 / 28 / 09$ 15:10 & $15: 12$ & 5714215 & Normal & OK & Normal $\left(24^{\circ} \mathrm{C}\right)$ & Normal (54\%) & OK & OK & 128 & -89.014444 & 41.367500 \\
\hline $8 / 28 / 09$ 15:15 & $15: 18$ & 5714215 & Normal & OK & Normal $\left(24^{\circ} \mathrm{C}\right)$ & Normal (54\%) & OK & OK & 128 & -89.058333 & 41.413333 \\
\hline $8 / 28 / 09$ 15:20 & $15: 23$ & 5714215 & Normal & OK & Normal $\left(24^{\circ} \mathrm{C}\right)$ & Normal (54\%) & OK & OK & 128 & -89.050833 & 41.460278 \\
\hline $8 / 28 / 09$ 15:25 & $15: 29$ & 5714215 & Normal & OK & Normal $\left(24^{\circ} \mathrm{C}\right)$ & Normal (54\%) & OK & OK & 128 & -89.058333 & 41.375833 \\
\hline $8 / 28 / 09$ 15:30 & $15: 35$ & 5714215 & Normal & OK & Normal $\left(24^{\circ} \mathrm{C}\right)$ & Normal (54\%) & OK & OK & 128 & -89.125833 & 41.368611 \\
\hline $8 / 28 / 09$ 15:35 & $15: 40$ & 5714215 & Normal & OK & Normal $\left(24^{\circ} \mathrm{C}\right)$ & Normal (54\%) & OK & OK & 128 & -89.125833 & 41.368611 \\
\hline $8 / 28 / 09$ 15:40 & $15: 46$ & 5714215 & Normal & OK & Normal $\left(24^{\circ} \mathrm{C}\right)$ & Normal (54\%) & OK & OK & 128 & -89.125833 & 41.368611 \\
\hline $8 / 28 / 09$ 15:45 & $15: 51$ & 5714215 & Normal & OK & Normal $\left(24^{\circ} \mathrm{C}\right)$ & Normal (54\%) & OK & OK & 128 & -89.200000 & 41.364444 \\
\hline $8 / 28 / 09$ 15:50 & $15: 57$ & 5714215 & Normal & OK & Normal $\left(24^{\circ} \mathrm{C}\right)$ & Normal (54\%) & OK & OK & 128 & -89.240556 & 41.371667 \\
\hline $8 / 28 / 09$ 16:00 & $16: 03$ & 5714215 & Normal & OK & Normal $\left(24^{\circ} \mathrm{C}\right)$ & Normal (54\%) & OK & OK & 128 & -89.458333 & 41.400833 \\
\hline $8 / 28 / 09$ 16:05 & $16: 13$ & 5714215 & Normal & OK & Normal $\left(24^{\circ} \mathrm{C}\right)$ & Normal (54\%) & OK & OK & 128 & -89.558333 & 41.393611 \\
\hline $8 / 28 / 0916: 11$ & $16: 14$ & 5714215 & Normal & OK & Normal $\left(24^{\circ} \mathrm{C}\right)$ & Normal (54\%) & OK & OK & 128 & -89.558333 & 41.393611 \\
\hline $8 / 28 / 09$ 16:15 & $16: 19$ & 5714215 & Normal & OK & Normal $\left(24^{\circ} \mathrm{C}\right)$ & Normal (54\%) & OK & OK & 128 & -89.558333 & 41.393611 \\
\hline $8 / 28 / 09$ 16:20 & $16: 25$ & 5714215 & Normal & OK & Normal $\left(24^{\circ} \mathrm{C}\right)$ & Normal (54\%) & OK & OK & 128 & -89.558333 & 41.393611 \\
\hline $8 / 28 / 0916: 25$ & $16: 30$ & 5714215 & Normal & OK & Normal $\left(24^{\circ} \mathrm{C}\right)$ & Normal (54\%) & OK & OK & 128 & -89.623889 & 41.394722 \\
\hline $8 / 28 / 09$ 16:30 & $16: 36$ & 5714215 & Normal & OK & Normal $\left(24^{\circ} \mathrm{C}\right)$ & Normal (54\%) & OK & OK & 128 & -89.725833 & 41.387500 \\
\hline $8 / 28 / 09$ 16:35 & $16: 41$ & 5714215 & Normal & OK & Normal $\left(24^{\circ} \mathrm{C}\right)$ & Normal (54\%) & OK & OK & 128 & -89.834167 & 41.388333 \\
\hline $8 / 28 / 09$ 16:40 & $16: 47$ & 5714215 & Normal & OK & Normal $\left(24^{\circ} \mathrm{C}\right)$ & Normal (54\%) & OK & OK & 128 & -89.941667 & 41.416667 \\
\hline $8 / 28 / 09$ 16:45 & $16: 52$ & 5714215 & Normal & OK & Normal $\left(24^{\circ} \mathrm{C}\right)$ & Normal (54\%) & OK & OK & 128 & -90.050000 & 41.413333 \\
\hline $8 / 28 / 09$ 16:50 & $16: 52$ & 5714215 & Normal & OK & Normal $\left(24^{\circ} \mathrm{C}\right)$ & Normal (54\%) & OK & OK & 128 & -90.157222 & 41.430000 \\
\hline 8/28/09 16:55 & $16: 58$ & 5714215 & Normal & OK & Normal $\left(24^{\circ} \mathrm{C}\right)$ & Normal (54\%) & OK & OK & 128 & -90.267500 & 41.440556 \\
\hline $8 / 28 / 09$ 17:00 & 17:09 & 5714215 & Normal & OK & Normal $\left(24^{\circ} \mathrm{C}\right)$ & Normal (54\%) & OK & OK & 128 & -90.338333 & 41.476944 \\
\hline 8/28/09 17:05 & $17: 15$ & 5714215 & Normal & OK & Normal $\left(24^{\circ} \mathrm{C}\right)$ & Normal (54\%) & OK & OK & 128 & -90.334167 & 41.486389 \\
\hline 8/28/09 17:09 & $17: 15$ & 5714215 & Alarm & Open & Normal $\left(25^{\circ} \mathrm{C}\right)$ & Normal (53\%) & OK & OK & 128 & -90.334167 & 41.486389 \\
\hline $8 / 28 / 09$ 17:11 & $17: 20$ & 5714215 & Alarm & Open & Normal $\left(25^{\circ} \mathrm{C}\right)$ & Normal (53\%) & OK & OK & 128 & -90.334167 & 41.486389 \\
\hline $8 / 28 / 09$ 17:15 & $17: 26$ & 5714215 & Normal & OK & Normal $\left(25^{\circ} \mathrm{C}\right)$ & Normal (53\%) & OK & OK & 128 & -90.334167 & 41.486389 \\
\hline 8/28/09 17:20 & $17: 31$ & 5714215 & Normal & OK & Normal $\left(25^{\circ} \mathrm{C}\right)$ & Normal (53\%) & OK & OK & 128 & -90.334167 & 41.486389 \\
\hline $8 / 28 / 09$ 17:25 & $17: 37$ & 5714215 & Normal & OK & Normal $\left(25^{\circ} \mathrm{C}\right)$ & Normal (53\%) & OK & OK & 128 & -90.340556 & 41.511389 \\
\hline 8/28/09 17:30 & $17: 42$ & 5714215 & Normal & OK & Normal $\left(25^{\circ} \mathrm{C}\right)$ & Normal (53\%) & OK & OK & 128 & -90.234167 & 41.562500 \\
\hline $8 / 28 / 09$ 17:35 & $17: 42$ & 5714215 & Normal & OK & Normal $\left(25^{\circ} \mathrm{C}\right)$ & Normal (53\%) & OK & OK & 128 & -90.209167 & 41.581111 \\
\hline $8 / 28 / 09$ 17:40 & $17: 48$ & 5714215 & Normal & OK & Normal $\left(25^{\circ} \mathrm{C}\right)$ & Normal (53\%) & OK & OK & 128 & -90.163333 & 41.642500 \\
\hline $8 / 28 / 09$ 17:45 & $17: 53$ & 5714215 & Normal & OK & Normal $\left(25^{\circ} \mathrm{C}\right)$ & Normal (53\%) & OK & OK & 128 & -90.080000 & 41.690556 \\
\hline $8 / 28 / 09$ 17:50 & $17: 59$ & 5714215 & Normal & OK & Normal $\left(25^{\circ} \mathrm{C}\right)$ & Normal (53\%) & OK & OK & 128 & -89.983333 & 41.718611 \\
\hline $8 / 28 / 09$ 17:55 & $18: 04$ & 5714215 & Normal & OK & Normal $\left(26^{\circ} \mathrm{C}\right)$ & Normal (53\%) & OK & OK & 128 & -89.876944 & 41.744722 \\
\hline
\end{tabular}




\begin{tabular}{|c|c|c|c|c|c|c|c|c|c|c|c|}
\hline Event Time & at QC Hub & Tag ID & Tag Status & Seal & Temperature & Humidity & Shock & Battery & RSSI & Longitude & Latitude \\
\hline $8 / 28 / 0918: 00$ & $18: 10$ & 5714215 & Normal & OK & $\operatorname{Normal}\left(26^{\circ} \mathrm{C}\right)$ & Normal (53\%) & OK & OK & 128 & -89.775000 & 41.757222 \\
\hline 8/28/09 18:05 & $18: 15$ & 5714215 & Normal & OK & Normal $\left(26^{\circ} \mathrm{C}\right)$ & Normal (53\%) & OK & OK & 128 & -89.689444 & 41.750833 \\
\hline $8 / 28 / 09$ 18:10 & $18: 21$ & 5714215 & Normal & OK & $\operatorname{Normal}\left(26^{\circ} \mathrm{C}\right)$ & Normal (53\%) & OK & OK & 128 & -89.689444 & 41.762500 \\
\hline 8/28/09 18:15 & $18: 26$ & 5714215 & Normal & OK & Normal $\left(26^{\circ} \mathrm{C}\right)$ & Normal (53\%) & OK & OK & 128 & -89.678056 & 41.750833 \\
\hline $8 / 28 / 09$ 18:20 & $18: 32$ & 5714215 & Normal & $\mathrm{OK}$ & Normal $\left(26^{\circ} \mathrm{C}\right)$ & Normal (53\%) & OK & OK & 128 & -89.635278 & 41.759167 \\
\hline $8 / 28 / 0918: 25$ & $18: 38$ & 5714215 & Normal & OK & Normal $\left(26^{\circ} \mathrm{C}\right)$ & Normal (53\%) & OK & OK & 128 & -89.532222 & 41.789444 \\
\hline $8 / 28 / 09$ 18:30 & $18: 38$ & 5714215 & Normal & OK & Normal $\left(26^{\circ} \mathrm{C}\right)$ & Normal (53\%) & OK & OK & 128 & -89.432222 & 41.822778 \\
\hline 8/28/09 18:35 & $18: 43$ & 5714215 & Normal & OK & Normal $\left(26^{\circ} \mathrm{C}\right)$ & Normal (53\%) & OK & OK & 128 & -89.336389 & 41.822778 \\
\hline $8 / 28 / 0918: 40$ & $18: 48$ & 5714215 & Normal & OK & Normal $\left(26^{\circ} \mathrm{C}\right)$ & Normal (53\%) & OK & OK & 128 & -89.231111 & 41.850833 \\
\hline $8 / 28 / 09$ 18:45 & $18: 54$ & 5714215 & Normal & OK & Normal $\left(26^{\circ} \mathrm{C}\right)$ & Normal (53\%) & OK & OK & 128 & -89.125000 & 41.883333 \\
\hline 8/28/09 18:50 & $19: 00$ & 5714215 & Normal & OK & $\operatorname{Normal}\left(26^{\circ} \mathrm{C}\right)$ & Normal (53\%) & OK & OK & 128 & -89.025000 & 41.904167 \\
\hline 8/28/09 18:55 & 19:05 & 5714215 & Normal & OK & Normal $\left(26^{\circ} \mathrm{C}\right)$ & Normal (53\%) & OK & OK & 128 & -88.913333 & 41.900000 \\
\hline 8/28/09 19:00 & 19:11 & 5714215 & Normal & OK & Normal $\left(26^{\circ} \mathrm{C}\right)$ & Normal (53\%) & OK & OK & 128 & -88.814444 & 41.900000 \\
\hline 8/28/09 19:05 & 19:16 & 5714215 & Normal & OK & Normal $\left(26^{\circ} \mathrm{C}\right)$ & Normal (53\%) & OK & OK & 128 & -88.739444 & 41.900000 \\
\hline 8/28/09 19:10 & $19: 22$ & 5714215 & Normal & OK & Normal $\left(26^{\circ} \mathrm{C}\right)$ & Normal (52\%) & OK & OK & 128 & -88.739444 & 41.900000 \\
\hline 8/28/09 19:15 & $19: 27$ & 5714215 & Normal & OK & Normal $\left(26^{\circ} \mathrm{C}\right)$ & Normal (52\%) & OK & OK & 128 & -88.737500 & 41.900000 \\
\hline $8 / 28 / 09$ 19:20 & $19: 33$ & 5714215 & Normal & OK & $\operatorname{Normal}\left(26^{\circ} \mathrm{C}\right)$ & Normal (52\%) & OK & OK & 128 & -88.737500 & 41.900000 \\
\hline 8/28/09 19:25 & $19: 38$ & 5714215 & Normal & OK & Normal $\left(26^{\circ} \mathrm{C}\right)$ & Normal (52\%) & OK & OK & 128 & -88.639444 & 41.894722 \\
\hline $8 / 28 / 0919: 30$ & 19:38 & 5714215 & Normal & OK & Normal $\left(26^{\circ} \mathrm{C}\right)$ & Normal (52\%) & OK & OK & 128 & -88.538333 & 41.859167 \\
\hline $8 / 28 / 09$ 19:35 & $19: 44$ & 5714215 & Normal & OK & $\operatorname{Normal}\left(26^{\circ} \mathrm{C}\right)$ & Normal (52\%) & OK & OK & 128 & -88.448889 & 41.812500 \\
\hline $8 / 28 / 0919: 40$ & $19: 49$ & 5714215 & Normal & OK & Normal $\left(26^{\circ} \mathrm{C}\right)$ & Normal (52\%) & OK & OK & 128 & -88.347778 & 41.794722 \\
\hline $8 / 28 / 09$ 19:45 & $19: 55$ & 5714215 & Normal & OK & Normal $\left(26^{\circ} \mathrm{C}\right)$ & Normal (52\%) & OK & OK & 128 & -88.261389 & 41.800000 \\
\hline $8 / 28 / 09$ 19:50 & $20: 00$ & 5714215 & Normal & OK & Normal $\left(26^{\circ} \mathrm{C}\right)$ & Normal (52\%) & OK & OK & 128 & -88.156111 & 41.806111 \\
\hline $8 / 28 / 09$ 19:55 & $20: 06$ & 5714215 & Normal & OK & Normal $\left(26^{\circ} \mathrm{C}\right)$ & Normal (52\%) & OK & OK & 128 & -88.062500 & 41.810278 \\
\hline $8 / 28 / 0920: 00$ & 20:11 & 5714215 & Normal & OK & Normal $\left(26^{\circ} \mathrm{C}\right)$ & Normal (52\%) & OK & OK & 128 & -88.054167 & 41.807222 \\
\hline $8 / 28 / 0920: 05$ & $20: 22$ & 5714215 & Normal & OK & Normal $\left(26^{\circ} \mathrm{C}\right)$ & Normal (54\%) & OK & OK & 128 & -88.054167 & 41.781111 \\
\hline $8 / 28 / 0920: 10$ & $20: 11$ & 5714215 & Normal & OK & Normal $\left(26^{\circ} \mathrm{C}\right)$ & Normal (54\%) & OK & OK & 128 & -88.035278 & 41.732222 \\
\hline $8 / 28 / 0913: 40$ & $13: 44$ & 5714220 & Normal & $\overline{O K}$ & $\operatorname{Normal}\left(23^{\circ} \mathrm{C}\right)$ & $\operatorname{Normal}(57 \%)$ & OK & OK & 131 & -87.971667 & 41.732222 \\
\hline $8 / 28 / 0913: 45$ & $13: 49$ & 5714220 & Normal & OK & Normal $\left(23^{\circ} \mathrm{C}\right)$ & Normal (57\%) & OK & OK & 131 & -87.971667 & 41.732222 \\
\hline $8 / 28 / 09$ 13:50 & $14: 00$ & 5714220 & Normal & OK & Normal $\left(23^{\circ} \mathrm{C}\right)$ & Normal (57\%) & OK & OK & 131 & -87.971667 & 41.732222 \\
\hline $8 / 28 / 0913: 55$ & $14: 00$ & 5714220 & Normal & OK & Normal $\left(23^{\circ} \mathrm{C}\right)$ & Normal (57\%) & OK & OK & 131 & -87.971667 & 41.732222 \\
\hline $8 / 28 / 09$ 14:00 & $14: 06$ & 5714220 & Normal & OK & Normal $\left(23^{\circ} \mathrm{C}\right)$ & Normal (57\%) & OK & OK & 131 & -88.068611 & 41.684167 \\
\hline $8 / 28 / 09$ 14:05 & $14: 11$ & 5714220 & Normal & OK & Normal $\left(23^{\circ} \mathrm{C}\right)$ & Normal (57\%) & OK & OK & 131 & -88.154167 & 41.640556 \\
\hline $8 / 28 / 09$ 14:10 & $14: 17$ & 5714220 & Normal & OK & Normal $\left(23^{\circ} \mathrm{C}\right)$ & Normal (57\%) & OK & OK & 131 & -88.181111 & 41.568611 \\
\hline $8 / 28 / 09$ 14:15 & $14: 22$ & 5714220 & Normal & OK & Normal $\left(23^{\circ} \mathrm{C}\right)$ & Normal (57\%) & OK & OK & 131 & -88.197778 & 41.493611 \\
\hline $8 / 28 / 0914: 20$ & $14: 22$ & 5714220 & Normal & OK & Normal $\left(23^{\circ} \mathrm{C}\right)$ & Normal (57\%) & OK & OK & 131 & -88.280000 & 41.462500 \\
\hline $8 / 28 / 09$ 14:25 & $14: 28$ & 5714220 & Normal & OK & Normal $\left(23^{\circ} \mathrm{C}\right)$ & Normal (57\%) & OK & OK & 131 & -88.371667 & 41.417500 \\
\hline $8 / 28 / 09$ 14:32 & $14: 39$ & 5714220 & Normal & OK & Normal $\left(23^{\circ} \mathrm{C}\right)$ & Normal (57\%) & OK & OK & 131 & -88.422778 & 41.392500 \\
\hline $8 / 28 / 09$ 14:35 & $14: 39$ & 5714220 & Normal & OK & Normal $\left(23^{\circ} \mathrm{C}\right)$ & Normal (57\%) & OK & OK & 131 & -88.422778 & 41.392500 \\
\hline $8 / 28 / 0914: 40$ & $14: 44$ & 5714220 & Normal & OK & Normal $\left(23^{\circ} \mathrm{C}\right)$ & Normal (57\%) & OK & OK & 131 & -88.422778 & 41.392500 \\
\hline $8 / 28 / 09$ 14:45 & $14: 50$ & 5714220 & Normal & OK & Normal $\left(23^{\circ} \mathrm{C}\right)$ & Normal $(57 \%)$ & OK & OK & 131 & -88.461389 & 41.378056 \\
\hline $8 / 28 / 09$ 14:50 & $14: 56$ & 5714220 & Normal & OK & Normal $\left(23^{\circ} \mathrm{C}\right)$ & Normal $(57 \%)$ & OK & OK & 131 & -88.575000 & 41.376944 \\
\hline $8 / 28 / 09$ 14:55 & $15: 01$ & 5714220 & Normal & OK & Normal $\left(24^{\circ} \mathrm{C}\right)$ & Normal (57\%) & OK & OK & 131 & -88.717500 & 41.375833 \\
\hline $8 / 28 / 09$ 15:00 & $15: 07$ & 5714220 & Normal & OK & Normal $\left(24^{\circ} \mathrm{C}\right)$ & Normal $(57 \%)$ & OK & OK & 131 & -88.792500 & 41.376944 \\
\hline $8 / 28 / 09$ 15:05 & $15: 12$ & 5714220 & Normal & OK & Normal $\left(24^{\circ} \mathrm{C}\right)$ & Normal (57\%) & OK & OK & 131 & -88.901944 & 41.368611 \\
\hline $8 / 28 / 09$ 15:10 & $15: 12$ & 5714220 & Normal & OK & Normal $\left(24^{\circ} \mathrm{C}\right)$ & Normal (57\%) & OK & OK & 131 & -89.014444 & 41.367500 \\
\hline $8 / 28 / 09$ 15:15 & $15: 18$ & 5714220 & Normal & OK & Normal $\left(24^{\circ} \mathrm{C}\right)$ & Normal (57\%) & OK & OK & 131 & -89.058333 & 41.413333 \\
\hline $8 / 28 / 09$ 15:20 & $15: 23$ & 5714220 & Normal & OK & Normal $\left(24^{\circ} \mathrm{C}\right)$ & Normal (57\%) & OK & OK & 131 & -89.050833 & 41.460278 \\
\hline $8 / 28 / 09$ 15:25 & $15: 29$ & 5714220 & Normal & OK & Normal $\left(24^{\circ} \mathrm{C}\right)$ & Normal (57\%) & OK & OK & 131 & -89.058333 & 41.375833 \\
\hline $8 / 28 / 09$ 15:30 & $15: 35$ & 5714220 & Normal & OK & Normal $\left(24^{\circ} \mathrm{C}\right)$ & Normal (57\%) & OK & OK & 131 & -89.125833 & 41.368611 \\
\hline $8 / 28 / 09$ 15:35 & $15: 40$ & 5714220 & Normal & OK & Normal $\left(24^{\circ} \mathrm{C}\right)$ & Normal (57\%) & OK & OK & 131 & -89.125833 & 41.368611 \\
\hline $8 / 28 / 0915: 40$ & $15: 46$ & 5714220 & Normal & OK & Normal $\left(24^{\circ} \mathrm{C}\right)$ & Normal (57\%) & OK & OK & 131 & -89.125833 & 41.368611 \\
\hline $8 / 28 / 09$ 15:45 & $15: 51$ & 5714220 & Normal & OK & Normal $\left(24^{\circ} \mathrm{C}\right)$ & Normal (57\%) & OK & OK & 131 & -89.200000 & 41.364444 \\
\hline $8 / 28 / 09$ 15:50 & $15: 57$ & 5714220 & Normal & OK & Normal $\left(24^{\circ} \mathrm{C}\right)$ & Normal (57\%) & OK & OK & 131 & -89.240556 & 41.371667 \\
\hline $8 / 28 / 09$ 16:00 & $16: 03$ & 5714220 & Normal & OK & Normal $\left(24^{\circ} \mathrm{C}\right)$ & Normal $(57 \%)$ & OK & OK & 131 & -89.458333 & 41.400833 \\
\hline $8 / 28 / 09$ 16:05 & $16: 13$ & 5714220 & Normal & OK & Normal $\left(24^{\circ} \mathrm{C}\right)$ & Normal $(57 \%)$ & OK & OK & 131 & -89.558333 & 41.393611 \\
\hline 8/28/09 16:11 & $16: 14$ & 5714220 & Normal & OK & Normal $\left(24^{\circ} \mathrm{C}\right)$ & Normal (57\%) & OK & OK & 131 & -89.558333 & 41.393611 \\
\hline $8 / 28 / 0916: 15$ & $16: 19$ & 5714220 & Normal & OK & Normal $\left(24^{\circ} \mathrm{C}\right)$ & Normal (57\%) & OK & OK & 131 & -89.558333 & 41.393611 \\
\hline $8 / 28 / 0916: 20$ & $16: 25$ & 5714220 & Normal & OK & Normal $\left(24^{\circ} \mathrm{C}\right)$ & Normal (57\%) & OK & OK & 131 & -89.558333 & 41.393611 \\
\hline $8 / 28 / 09$ 16:25 & $16: 30$ & 5714220 & Normal & OK & Normal $\left(24^{\circ} \mathrm{C}\right)$ & Normal (57\%) & OK & OK & 131 & -89.623889 & 41.394722 \\
\hline $8 / 28 / 0916: 30$ & $16: 36$ & 5714220 & Normal & OK & Normal $\left(24^{\circ} \mathrm{C}\right)$ & Normal (57\%) & OK & OK & 131 & -89.725833 & 41.387500 \\
\hline $8 / 28 / 09$ 16:35 & $16: 41$ & 5714220 & Normal & OK & Normal $\left(24^{\circ} \mathrm{C}\right)$ & Normal (57\%) & OK & OK & 131 & -89.834167 & 41.388333 \\
\hline $8 / 28 / 0916: 40$ & $16: 47$ & 5714220 & Normal & OK & Normal $\left(24^{\circ} \mathrm{C}\right)$ & Normal (57\%) & OK & OK & 131 & -89.941667 & 41.416667 \\
\hline $8 / 28 / 0916: 45$ & $16: 52$ & 5714220 & Normal & OK & Normal $\left(24^{\circ} \mathrm{C}\right)$ & Normal (57\%) & OK & OK & 131 & -90.050000 & 41.413333 \\
\hline $8 / 28 / 09$ 16:50 & $16: 52$ & 5714220 & Normal & OK & Normal $\left(24^{\circ} \mathrm{C}\right)$ & Normal (57\%) & OK & OK & 131 & -90.157222 & 41.430000 \\
\hline $8 / 28 / 0916: 55$ & $16: 58$ & 5714220 & Normal & OK & Normal $\left(24^{\circ} \mathrm{C}\right)$ & Normal (57\%) & OK & OK & 131 & -90.267500 & 41.440556 \\
\hline 8/28/09 17:00 & $17: 09$ & 5714220 & Normal & OK & Normal $\left(24^{\circ} \mathrm{C}\right)$ & Normal (57\%) & OK & OK & 131 & -90.338333 & 41.476944 \\
\hline 8/28/09 17:05 & $17: 09$ & 5714220 & Normal & OK & Normal $\left(24^{\circ} \mathrm{C}\right)$ & Normal (57\%) & OK & OK & 131 & -90.334167 & 41.486389 \\
\hline $8 / 28 / 09$ 17:11 & $17: 15$ & 5714220 & Normal & OK & Normal $\left(24^{\circ} \mathrm{C}\right)$ & Normal $(57 \%)$ & OK & OK & 131 & -90.334167 & 41.486389 \\
\hline $8 / 28 / 09$ 17:15 & $17: 20$ & 5714220 & Normal & OK & Normal $\left(24^{\circ} \mathrm{C}\right)$ & Normal $(57 \%)$ & OK & OK & 131 & -90.334167 & 41.486389 \\
\hline $8 / 28 / 09$ 17:20 & $17: 26$ & 5714220 & Normal & OK & Normal $\left(24^{\circ} \mathrm{C}\right)$ & Normal (57\%) & OK & OK & 131 & -90.334167 & 41.486389 \\
\hline $8 / 28 / 09$ 17:25 & $17: 31$ & 5714220 & Normal & OK & Normal $\left(24^{\circ} \mathrm{C}\right)$ & Normal (57\%) & OK & OK & 131 & -90.340556 & 41.511389 \\
\hline $8 / 28 / 0917: 30$ & $17: 37$ & 5714220 & Normal & OK & Normal $\left(24^{\circ} \mathrm{C}\right)$ & Normal (57\%) & OK & OK & 131 & -90.234167 & 41.562500 \\
\hline $8 / 28 / 09$ 17:35 & $17: 42$ & 5714220 & Normal & OK & Normal $\left(24^{\circ} \mathrm{C}\right)$ & Normal (57\%) & OK & OK & 131 & -90.209167 & 41.581111 \\
\hline $8 / 28 / 0917: 40$ & $17: 42$ & 5714220 & Normal & OK & Normal $\left(24^{\circ} \mathrm{C}\right)$ & Normal (57\%) & OK & OK & 131 & -90.163333 & 41.642500 \\
\hline $8 / 28 / 09$ 17:45 & $17: 48$ & 5714220 & Normal & OK & Normal $\left(24^{\circ} \mathrm{C}\right)$ & Normal (57\%) & OK & OK & 131 & -90.080000 & 41.690556 \\
\hline $8 / 28 / 09$ 17:50 & $17: 53$ & 5714220 & Normal & OK & Normal $\left(24^{\circ} \mathrm{C}\right)$ & Normal $(57 \%)$ & OK & OK & 131 & -89.983333 & 41.718611 \\
\hline $8 / 28 / 09$ 17:55 & $17: 59$ & 5714220 & Normal & OK & Normal $\left(25^{\circ} \mathrm{C}\right)$ & Normal (56\%) & OK & OK & 131 & -89.876944 & 41.744722 \\
\hline 8/28/09 18:00 & $18: 04$ & 5714220 & Normal & OK & Normal $\left(25^{\circ} \mathrm{C}\right)$ & Normal (56\%) & OK & OK & 131 & -89.775000 & 41.757222 \\
\hline $8 / 28 / 09$ 18:05 & $18: 10$ & 5714220 & Normal & OK & Normal $\left(25^{\circ} \mathrm{C}\right)$ & Normal (56\%) & OK & OK & 131 & -89.689444 & 41.750833 \\
\hline $8 / 28 / 09$ 18:10 & $18: 15$ & 5714220 & Normal & OK & Normal $\left(25^{\circ} \mathrm{C}\right)$ & Normal (56\%) & OK & OK & 131 & -89.689444 & 41.762500 \\
\hline
\end{tabular}




\begin{tabular}{|c|c|c|c|c|c|c|c|c|c|c|c|}
\hline Event Time & at QC Hub & Tag ID & Tag Status & Seal & Temperature & Humidity & Shock & Battery & RSSI & Longitude & Latitude \\
\hline 8/28/09 18:15 & $18: 21$ & 5714220 & Normal & OK & Normal $\left(25^{\circ} \mathrm{C}\right)$ & Normal (56\%) & OK & OK & 131 & -89.678056 & 41.750833 \\
\hline $8 / 28 / 09$ 18:20 & $18: 26$ & 5714220 & Normal & OK & Normal $\left(25^{\circ} \mathrm{C}\right)$ & Normal $(56 \%)$ & OK & $\mathrm{OK}$ & 131 & -89.635278 & 41.759167 \\
\hline $8 / 28 / 0918: 25$ & $18: 32$ & 5714220 & Normal & OK & Normal $\left(25^{\circ} \mathrm{C}\right)$ & Normal (56\%) & OK & $\mathrm{OK}$ & 131 & -89.532222 & 41.789444 \\
\hline 8/28/09 18:30 & $18: 38$ & 5714220 & Normal & OK & Normal $\left(25^{\circ} \mathrm{C}\right)$ & Normal (56\%) & OK & OK & 131 & -89.432222 & 41.822778 \\
\hline 8/28/09 18:35 & $18: 38$ & 5714220 & Normal & OK & Normal $\left(25^{\circ} \mathrm{C}\right)$ & Normal $(56 \%)$ & $\mathrm{OK}$ & OK & 131 & -89.336389 & 41.822778 \\
\hline $8 / 28 / 0918: 40$ & $18: 43$ & 5714220 & Normal & OK & Normal $\left(25^{\circ} \mathrm{C}\right)$ & Normal (56\%) & OK & OK & 131 & -89.231111 & 41.850833 \\
\hline $8 / 28 / 09$ 18:45 & $18: 48$ & 5714220 & Normal & OK & Normal $\left(26^{\circ} \mathrm{C}\right)$ & Normal (55\%) & OK & OK & 131 & -89.125000 & 41.883333 \\
\hline 8/28/09 18:50 & $18: 54$ & 5714220 & Normal & $\mathrm{OK}$ & Normal $\left(26^{\circ} \mathrm{C}\right)$ & Normal (55\%) & OK & OK & 131 & -89.025000 & 41.904167 \\
\hline 8/28/09 18:55 & 19:00 & 5714220 & Normal & OK & Normal $\left(26^{\circ} \mathrm{C}\right)$ & Normal (55\%) & OK & OK & 131 & -88.913333 & 41.900000 \\
\hline 8/28/09 19:00 & 19:05 & 5714220 & Normal & OK & Normal $\left(26^{\circ} \mathrm{C}\right)$ & Normal (55\%) & OK & OK & 131 & -88.814444 & 41.900000 \\
\hline 8/28/09 19:05 & 19:11 & 5714220 & Normal & OK & Normal $\left(26^{\circ} \mathrm{C}\right)$ & Normal $(55 \%)$ & OK & OK & 131 & -88.739444 & 41.900000 \\
\hline 8/28/09 19:10 & 19:16 & 5714220 & Normal & OK & Normal $\left(26^{\circ} \mathrm{C}\right)$ & Normal $(55 \%)$ & OK & OK & 131 & -88.739444 & 41.900000 \\
\hline 8/28/09 19:15 & $19: 22$ & 5714220 & Normal & OK & Normal $\left(26^{\circ} \mathrm{C}\right)$ & Normal $(55 \%)$ & OK & OK & 131 & -88.737500 & 41.900000 \\
\hline $8 / 28 / 09$ 19:20 & $19: 27$ & 5714220 & Normal & OK & Normal $\left(26^{\circ} \mathrm{C}\right)$ & Normal (55\%) & OK & OK & 131 & -88.737500 & 41.900000 \\
\hline $8 / 28 / 09$ 19:25 & $19: 33$ & 5714220 & Normal & OK & Normal $\left(26^{\circ} \mathrm{C}\right)$ & Normal (55\%) & OK & OK & 131 & -88.639444 & 41.894722 \\
\hline 8/28/09 19:30 & 19:38 & 5714220 & Normal & OK & Normal $\left(26^{\circ} \mathrm{C}\right)$ & Normal (55\%) & OK & $\mathrm{OK}$ & 131 & -88.538333 & 41.859167 \\
\hline 8/28/09 19:35 & 19:38 & 5714220 & Normal & OK & Normal $\left(26^{\circ} \mathrm{C}\right)$ & Normal (55\%) & OK & $\mathrm{OK}$ & 131 & -88.448889 & 41.812500 \\
\hline $8 / 28 / 09$ 19:40 & $19: 44$ & 5714220 & Normal & OK & Normal $\left(26^{\circ} \mathrm{C}\right)$ & Normal $(55 \%)$ & OK & OK & 131 & -88.347778 & 41.794722 \\
\hline 8/28/09 19:45 & $19: 49$ & 5714220 & Normal & OK & Normal $\left(26^{\circ} \mathrm{C}\right)$ & Normal $(55 \%)$ & OK & OK & 131 & -88.261389 & 41.800000 \\
\hline 8/28/09 19:50 & $19: 55$ & 5714220 & Normal & OK & Normal $\left(26^{\circ} \mathrm{C}\right)$ & Normal (55\%) & OK & OK & 131 & -88.156111 & 41.806111 \\
\hline 8/28/09 19:55 & $20: 00$ & 5714220 & Normal & OK & Normal $\left(26^{\circ} \mathrm{C}\right)$ & Normal (55\%) & OK & OK & 131 & -88.062500 & 41.810278 \\
\hline $8 / 28 / 09$ 20:00 & 20:06 & 5714220 & Normal & OK & Normal $\left(26^{\circ} \mathrm{C}\right)$ & Normal (55\%) & OK & OK & 131 & -88.054167 & 41.807222 \\
\hline $8 / 28 / 09$ 20:05 & $20: 11$ & 5714220 & Normal & OK & Normal $\left(26^{\circ} \mathrm{C}\right)$ & Normal (55\%) & OK & OK & 131 & -88.054167 & 41.781111 \\
\hline 8/28/09 20:10 & $20: 22$ & 5714220 & Normal & OK & Normal $\left(26^{\circ} \mathrm{C}\right)$ & Normal (55\%) & OK & OK & 131 & -88.035278 & 41.732222 \\
\hline $8 / 28 / 0913: 40$ & $13: 44$ & 5714225 & Normal & OK & $\operatorname{Normal}\left(23^{\circ} \mathrm{C}\right)$ & Normal $(56 \%)$ & OK & OK & 141 & -87.971667 & 41.732222 \\
\hline $8 / 28 / 09$ 13:45 & $13: 49$ & 5714225 & Normal & OK & Normal $\left(23^{\circ} \mathrm{C}\right)$ & Normal (56\%) & OK & OK & 141 & -87.971667 & 41.732222 \\
\hline $8 / 28 / 09$ 13:50 & $14: 00$ & 5714225 & Normal & OK & Normal $\left(23^{\circ} \mathrm{C}\right)$ & Normal (56\%) & OK & OK & 141 & -87.971667 & 41.732222 \\
\hline $8 / 28 / 09$ 13:55 & $14: 00$ & 5714225 & Normal & OK & Normal $\left(23^{\circ} \mathrm{C}\right)$ & Normal (56\%) & OK & OK & 141 & -87.971667 & 41.732222 \\
\hline $8 / 28 / 09$ 14:00 & $14: 06$ & 5714225 & Normal & OK & Normal $\left(23^{\circ} \mathrm{C}\right)$ & Normal (56\%) & OK & OK & 141 & -88.068611 & 41.684167 \\
\hline 8/28/09 14:05 & $14: 11$ & 5714225 & Normal & OK & Normal $\left(23^{\circ} \mathrm{C}\right)$ & Normal (56\%) & OK & OK & 141 & -88.154167 & 41.640556 \\
\hline $8 / 28 / 09$ 14:10 & $14: 17$ & 5714225 & Normal & OK & Normal $\left(23^{\circ} \mathrm{C}\right)$ & Normal (56\%) & OK & OK & 141 & -88.181111 & 41.568611 \\
\hline 8/28/09 14:15 & $14: 22$ & 5714225 & Normal & OK & Normal $\left(23^{\circ} \mathrm{C}\right)$ & Normal (56\%) & OK & OK & 141 & -88.197778 & 41.493611 \\
\hline $8 / 28 / 09$ 14:20 & $14: 22$ & 5714225 & Normal & OK & Normal $\left(23^{\circ} \mathrm{C}\right)$ & Normal (56\%) & OK & OK & 141 & -88.280000 & 41.462500 \\
\hline $8 / 28 / 09$ 14:25 & $14: 28$ & 5714225 & Normal & OK & Normal $\left(23^{\circ} \mathrm{C}\right)$ & Normal (56\%) & OK & OK & 141 & -88.371667 & 41.417500 \\
\hline $8 / 28 / 09$ 14:32 & $14: 39$ & 5714225 & Normal & OK & Normal $\left(23^{\circ} \mathrm{C}\right)$ & Normal (56\%) & OK & OK & 141 & -88.422778 & 41.392500 \\
\hline $8 / 28 / 09$ 14:35 & $14: 39$ & 5714225 & Normal & OK & Normal $\left(23^{\circ} \mathrm{C}\right)$ & Normal (56\%) & OK & OK & 141 & -88.422778 & 41.392500 \\
\hline $8 / 28 / 0914: 40$ & $14: 44$ & 5714225 & Normal & OK & Normal $\left(23^{\circ} \mathrm{C}\right)$ & Normal $(56 \%)$ & OK & OK & 141 & -88.422778 & 41.392500 \\
\hline $8 / 28 / 09$ 14:45 & $14: 50$ & 5714225 & Normal & OK & Normal $\left(23^{\circ} \mathrm{C}\right)$ & Normal $(56 \%)$ & OK & OK & 141 & -88.461389 & 41.378056 \\
\hline $8 / 28 / 09$ 14:50 & $14: 56$ & 5714225 & Normal & OK & Normal $\left(23^{\circ} \mathrm{C}\right)$ & Normal $(56 \%)$ & OK & OK & 141 & -88.575000 & 41.376944 \\
\hline $8 / 28 / 09$ 14:55 & $15: 01$ & 5714225 & Normal & OK & Normal $\left(23^{\circ} \mathrm{C}\right)$ & Normal (56\%) & OK & OK & 141 & -88.717500 & 41.375833 \\
\hline $8 / 28 / 09$ 15:00 & $15: 07$ & 5714225 & Normal & OK & Normal $\left(23^{\circ} \mathrm{C}\right)$ & Normal (56\%) & OK & OK & 141 & -88.792500 & 41.376944 \\
\hline $8 / 28 / 09$ 15:05 & $15: 12$ & 5714225 & Normal & OK & Normal $\left(23^{\circ} \mathrm{C}\right)$ & Normal (56\%) & OK & OK & 141 & -88.901944 & 41.368611 \\
\hline $8 / 28 / 09$ 15:10 & $15: 12$ & 5714225 & Normal & OK & Normal $\left(23^{\circ} \mathrm{C}\right)$ & Normal (56\%) & OK & OK & 141 & -89.014444 & 41.367500 \\
\hline $8 / 28 / 09$ 15:15 & $15: 18$ & 5714225 & Normal & OK & Normal $\left(23^{\circ} \mathrm{C}\right)$ & Normal (56\%) & OK & OK & 141 & -89.058333 & 41.413333 \\
\hline $8 / 28 / 09$ 15:20 & $15: 23$ & 5714225 & Normal & OK & Normal $\left(23^{\circ} \mathrm{C}\right)$ & Normal (56\%) & OK & OK & 141 & -89.050833 & 41.460278 \\
\hline $8 / 28 / 09$ 15:25 & $15: 29$ & 5714225 & Normal & OK & Normal $\left(23^{\circ} \mathrm{C}\right)$ & Normal (56\%) & OK & OK & 141 & -89.058333 & 41.375833 \\
\hline $8 / 28 / 09$ 15:30 & $15: 35$ & 5714225 & Normal & OK & Normal $\left(23^{\circ} \mathrm{C}\right)$ & Normal (56\%) & OK & OK & 141 & -89.125833 & 41.368611 \\
\hline $8 / 28 / 09$ 15:35 & $15: 40$ & 5714225 & Normal & OK & Normal $\left(23^{\circ} \mathrm{C}\right)$ & Normal (56\%) & OK & OK & 141 & -89.125833 & 41.368611 \\
\hline $8 / 28 / 09$ 15:40 & $15: 46$ & 5714225 & Normal & OK & Normal $\left(23^{\circ} \mathrm{C}\right)$ & Normal (56\%) & OK & OK & 141 & -89.125833 & 41.368611 \\
\hline $8 / 28 / 09$ 15:45 & $15: 51$ & 5714225 & Normal & OK & Normal $\left(23^{\circ} \mathrm{C}\right)$ & Normal (56\%) & OK & OK & 141 & -89.200000 & 41.364444 \\
\hline $8 / 28 / 09$ 15:50 & $15: 57$ & 5714225 & Normal & OK & Normal $\left(23^{\circ} \mathrm{C}\right)$ & Normal (56\%) & OK & OK & 141 & -89.240556 & 41.371667 \\
\hline $8 / 28 / 09$ 16:00 & $16: 03$ & 5714225 & Normal & OK & Normal $\left(23^{\circ} \mathrm{C}\right)$ & Normal $(56 \%)$ & OK & OK & 141 & -89.458333 & 41.400833 \\
\hline $8 / 28 / 09$ 16:05 & $16: 13$ & 5714225 & Normal & OK & Normal $\left(23^{\circ} \mathrm{C}\right)$ & Normal $(56 \%)$ & OK & OK & 141 & -89.558333 & 41.393611 \\
\hline $8 / 28 / 09$ 16:11 & $16: 14$ & 5714225 & Normal & OK & Normal $\left(23^{\circ} \mathrm{C}\right)$ & Normal (56\%) & OK & OK & 141 & -89.558333 & 41.393611 \\
\hline $8 / 28 / 09$ 16:15 & $16: 19$ & 5714225 & Normal & OK & Normal $\left(23^{\circ} \mathrm{C}\right)$ & Normal (56\%) & OK & OK & 141 & -89.558333 & 41.393611 \\
\hline $8 / 28 / 09$ 16:20 & $16: 25$ & 5714225 & Normal & OK & Normal $\left(23^{\circ} \mathrm{C}\right)$ & Normal (56\%) & OK & OK & 141 & -89.558333 & 41.393611 \\
\hline $8 / 28 / 09$ 16:25 & $16: 30$ & 5714225 & Normal & OK & Normal $\left(23^{\circ} \mathrm{C}\right)$ & Normal (56\%) & OK & OK & 141 & -89.623889 & 41.394722 \\
\hline $8 / 28 / 09$ 16:30 & $16: 36$ & 5714225 & Normal & OK & Normal $\left(23^{\circ} \mathrm{C}\right)$ & Normal (56\%) & OK & OK & 141 & -89.725833 & 41.387500 \\
\hline $8 / 28 / 09$ 16:35 & $16: 41$ & 5714225 & Normal & OK & Normal $\left(23^{\circ} \mathrm{C}\right)$ & Normal (56\%) & OK & OK & 141 & -89.834167 & 41.388333 \\
\hline $8 / 28 / 0916: 40$ & $16: 47$ & 5714225 & Normal & OK & Normal $\left(23^{\circ} \mathrm{C}\right)$ & Normal $(56 \%)$ & OK & OK & 141 & -89.941667 & 41.416667 \\
\hline $8 / 28 / 09$ 16:45 & $16: 52$ & 5714225 & Normal & OK & Normal $\left(23^{\circ} \mathrm{C}\right)$ & Normal (56\%) & OK & OK & 141 & -90.050000 & 41.413333 \\
\hline $8 / 28 / 0916: 50$ & $16: 52$ & 5714225 & Normal & OK & Normal $\left(23^{\circ} \mathrm{C}\right)$ & Normal $(56 \%)$ & OK & OK & 141 & -90.157222 & 41.430000 \\
\hline $8 / 28 / 09$ 16:55 & $16: 58$ & 5714225 & Normal & OK & Normal $\left(23^{\circ} \mathrm{C}\right)$ & Normal (56\%) & OK & OK & 141 & -90.267500 & 41.440556 \\
\hline $8 / 28 / 09$ 17:00 & $17: 09$ & 5714225 & Normal & OK & Normal $\left(23^{\circ} \mathrm{C}\right)$ & Normal (56\%) & OK & OK & 141 & -90.338333 & 41.476944 \\
\hline $8 / 28 / 09$ 17:05 & 17:09 & 5714225 & Normal & OK & Normal $\left(23^{\circ} \mathrm{C}\right)$ & Normal (56\%) & OK & OK & 141 & -90.334167 & 41.486389 \\
\hline $8 / 28 / 09$ 17:11 & $17: 15$ & 5714225 & Normal & OK & Normal $\left(23^{\circ} \mathrm{C}\right)$ & Normal $(56 \%)$ & OK & OK & 141 & -90.334167 & 41.486389 \\
\hline 8/28/09 17:15 & $17: 20$ & 5714225 & Normal & OK & Normal $\left(23^{\circ} \mathrm{C}\right)$ & Normal $(56 \%)$ & OK & OK & 141 & -90.334167 & 41.486389 \\
\hline $8 / 28 / 09$ 17:20 & $17: 26$ & 5714225 & Normal & OK & Normal $\left(23^{\circ} \mathrm{C}\right)$ & Normal (56\%) & OK & OK & 141 & -90.334167 & 41.486389 \\
\hline $8 / 28 / 09$ 17:25 & $17: 31$ & 5714225 & Normal & OK & Normal $\left(23^{\circ} \mathrm{C}\right)$ & Normal (56\%) & OK & OK & 141 & -90.340556 & 41.511389 \\
\hline $8 / 28 / 09$ 17:30 & $17: 37$ & 5714225 & Normal & OK & Normal $\left(23^{\circ} \mathrm{C}\right)$ & Normal (56\%) & OK & OK & 141 & -90.234167 & 41.562500 \\
\hline $8 / 28 / 09$ 17:35 & $17: 42$ & 5714225 & Normal & OK & Normal $\left(23^{\circ} \mathrm{C}\right)$ & Normal (56\%) & OK & OK & 141 & -90.209167 & 41.581111 \\
\hline $8 / 28 / 09$ 17:40 & $17: 42$ & 5714225 & Normal & OK & Normal $\left(23^{\circ} \mathrm{C}\right)$ & Normal (56\%) & OK & OK & 141 & -90.163333 & 41.642500 \\
\hline $8 / 28 / 09$ 17:45 & $17: 48$ & 5714225 & Normal & OK & Normal $\left(23^{\circ} \mathrm{C}\right)$ & Normal (56\%) & OK & OK & 141 & -90.080000 & 41.690556 \\
\hline $8 / 28 / 09$ 17:50 & $17: 53$ & 5714225 & Normal & OK & Normal $\left(23^{\circ} \mathrm{C}\right)$ & Normal (56\%) & OK & OK & 141 & -89.983333 & 41.718611 \\
\hline $8 / 28 / 09$ 17:55 & $17: 59$ & 5714225 & Normal & OK & Normal $\left(25^{\circ} \mathrm{C}\right)$ & Normal (56\%) & OK & OK & 141 & -89.876944 & 41.744722 \\
\hline 8/28/09 18:00 & $18: 04$ & 5714225 & Normal & OK & Normal $\left(25^{\circ} \mathrm{C}\right)$ & Normal (56\%) & OK & OK & 141 & -89.775000 & 41.757222 \\
\hline 8/28/09 18:05 & $18: 10$ & 5714225 & Normal & OK & Normal $\left(25^{\circ} \mathrm{C}\right)$ & Normal (56\%) & OK & OK & 141 & -89.689444 & 41.750833 \\
\hline $8 / 28 / 09$ 18:10 & $18: 15$ & 5714225 & Normal & OK & Normal $\left(25^{\circ} \mathrm{C}\right)$ & Normal (56\%) & OK & OK & 141 & -89.689444 & 41.762500 \\
\hline $8 / 28 / 0918: 15$ & $18: 21$ & 5714225 & Normal & OK & Normal $\left(25^{\circ} \mathrm{C}\right)$ & Normal (56\%) & OK & OK & 141 & -89.678056 & 41.750833 \\
\hline $8 / 28 / 0918: 20$ & $18: 26$ & 5714225 & Normal & OK & Normal $\left(25^{\circ} \mathrm{C}\right)$ & Normal $(56 \%)$ & OK & OK & 141 & -89.635278 & 41.759167 \\
\hline $8 / 28 / 0918: 25$ & $18: 32$ & 5714225 & Normal & OK & Normal $\left(25^{\circ} \mathrm{C}\right)$ & Normal $(56 \%)$ & OK & OK & 141 & -89.532222 & 41.789444 \\
\hline
\end{tabular}




\begin{tabular}{|c|c|c|c|c|c|c|c|c|c|c|c|}
\hline Event Time & at QC Hub & Tag ID & Tag Status & Seal & Temperature & Humidity & Shock & Battery & RSSI & Longitude & Latitude \\
\hline $8 / 28 / 09$ 18:30 & $18: 38$ & 5714225 & Normal & OK & Normal $\left(25^{\circ} \mathrm{C}\right)$ & Normal (56\%) & OK & OK & 141 & -89.432222 & 41.822778 \\
\hline $8 / 28 / 09$ 18:35 & $18: 38$ & 5714225 & Normal & OK & Normal $\left(25^{\circ} \mathrm{C}\right)$ & Normal (56\%) & OK & OK & 141 & -89.336389 & 41.822778 \\
\hline $8 / 28 / 0918: 40$ & $18: 43$ & 5714225 & Normal & OK & Normal $\left(25^{\circ} \mathrm{C}\right)$ & Normal $(56 \%)$ & $\mathrm{OK}$ & $\mathrm{OK}$ & 141 & -89.231111 & 41.850833 \\
\hline 8/28/09 18:45 & $18: 48$ & 5714225 & Normal & OK & Normal $\left(25^{\circ} \mathrm{C}\right)$ & Normal (55\%) & OK & OK & 141 & -89.125000 & 41.883333 \\
\hline 8/28/09 18:50 & $18: 54$ & 5714225 & Normal & OK & Normal $\left(25^{\circ} \mathrm{C}\right)$ & Normal (55\%) & $\mathrm{OK}$ & $\mathrm{OK}$ & 141 & -89.025000 & 41.904167 \\
\hline $8 / 28 / 09$ 18:55 & 19:00 & 5714225 & Normal & OK & Normal $\left(25^{\circ} \mathrm{C}\right)$ & Normal (55\%) & $\mathrm{OK}$ & $\mathrm{OK}$ & 141 & -88.913333 & 41.900000 \\
\hline 8/28/09 19:00 & 19:05 & 5714225 & Normal & OK & Normal $\left(25^{\circ} \mathrm{C}\right)$ & Normal (55\%) & OK & OK & 141 & -88.814444 & 41.900000 \\
\hline 8/28/09 19:05 & 19:11 & 5714225 & Normal & OK & Normal $\left(25^{\circ} \mathrm{C}\right)$ & Normal (55\%) & OK & OK & 141 & -88.739444 & 41.900000 \\
\hline 8/28/09 19:10 & $19: 16$ & 5714225 & Normal & OK & Normal $\left(25^{\circ} \mathrm{C}\right)$ & Normal (55\%) & OK & OK & 141 & -88.739444 & 41.900000 \\
\hline 8/28/09 19:15 & $19: 22$ & 5714225 & Normal & OK & Normal $\left(25^{\circ} \mathrm{C}\right)$ & Normal (55\%) & $\mathrm{OK}$ & $\mathrm{OK}$ & 141 & -88.737500 & 41.900000 \\
\hline 8/28/09 19:20 & $19: 27$ & 5714225 & Normal & $\mathrm{OK}$ & Normal $\left(25^{\circ} \mathrm{C}\right)$ & Normal (55\%) & OK & OK & 141 & -88.737500 & 41.900000 \\
\hline 8/28/09 19:25 & 19:33 & 5714225 & Normal & OK & Normal $\left(25^{\circ} \mathrm{C}\right)$ & Normal (55\%) & OK & OK & 141 & -88.639444 & 41.894722 \\
\hline 8/28/09 19:30 & $19: 38$ & 5714225 & Normal & OK & Normal $\left(25^{\circ} \mathrm{C}\right)$ & Normal (55\%) & OK & OK & 141 & -88.538333 & 41.859167 \\
\hline 8/28/09 19:35 & 19:38 & 5714225 & Normal & OK & Normal $\left(25^{\circ} \mathrm{C}\right)$ & Normal (55\%) & OK & OK & 141 & -88.448889 & 41.812500 \\
\hline 8/28/09 19:40 & $19: 44$ & 5714225 & Normal & OK & Normal $\left(25^{\circ} \mathrm{C}\right)$ & Normal (55\%) & OK & OK & 141 & -88.347778 & 41.794722 \\
\hline 8/28/09 19:45 & $19: 49$ & 5714225 & Normal & $\mathrm{OK}$ & Normal $\left(25^{\circ} \mathrm{C}\right)$ & Normal (55\%) & OK & OK & 141 & -88.261389 & 41.800000 \\
\hline 8/28/09 19:50 & 19:55 & 5714225 & Normal & OK & Normal $\left(25^{\circ} \mathrm{C}\right)$ & Normal (55\%) & OK & $\mathrm{OK}$ & 141 & -88.156111 & 41.806111 \\
\hline 8/28/09 19:55 & $20: 00$ & 5714225 & Normal & OK & Normal $\left(25^{\circ} \mathrm{C}\right)$ & Normal (55\%) & $\mathrm{OK}$ & $\mathrm{OK}$ & 141 & -88.062500 & 41.810278 \\
\hline $8 / 28 / 0920: 00$ & $20: 06$ & 5714225 & Normal & OK & Normal $\left(25^{\circ} \mathrm{C}\right)$ & Normal (55\%) & OK & OK & 141 & -88.054167 & 41.807222 \\
\hline 8/28/09 20:05 & $20: 11$ & 5714225 & Normal & OK & Normal $\left(25^{\circ} \mathrm{C}\right)$ & Normal (55\%) & $\mathrm{OK}$ & $\mathrm{OK}$ & 141 & -88.054167 & 41.781111 \\
\hline $8 / 28 / 09$ 20:10 & $20: 22$ & 5714225 & Normal & OK & Normal $\left(25^{\circ} \mathrm{C}\right)$ & Normal $(55 \%)$ & $\mathrm{OK}$ & $\mathrm{OK}$ & 141 & -88.035278 & 41.732222 \\
\hline $8 / 28 / 0913: 40$ & $13: 44$ & 5714230 & Normal & OK & Normal $\left(23^{\circ} \mathrm{C}\right)$ & Normal (56\%) & $\mathrm{OK}$ & OK & 127 & -87.971667 & 41.732222 \\
\hline 8/28/09 13:45 & $13: 49$ & 5714230 & Normal & OK & Normal $\left(23^{\circ} \mathrm{C}\right)$ & Normal (56\%) & OK & OK & 127 & -87.971667 & 41.732222 \\
\hline $8 / 28 / 0913: 50$ & $14: 00$ & 5714230 & Normal & OK & Normal $\left(23^{\circ} \mathrm{C}\right)$ & Normal (56\%) & OK & OK & 127 & -87.971667 & 41.732222 \\
\hline $8 / 28 / 09$ 13:55 & $14: 00$ & 5714230 & Normal & OK & Normal $\left(23^{\circ} \mathrm{C}\right)$ & Normal (56\%) & OK & OK & 127 & -87.971667 & 41.732222 \\
\hline $8 / 28 / 0914: 00$ & 14:06 & 5714230 & Normal & OK & Normal $\left(23^{\circ} \mathrm{C}\right)$ & Normal (56\%) & OK & $\mathrm{OK}$ & 127 & -88.068611 & 41.684167 \\
\hline $8 / 28 / 09$ 14:05 & $14: 11$ & 5714230 & Normal & OK & Normal $\left(23^{\circ} \mathrm{C}\right)$ & Normal (56\%) & OK & OK & 127 & -88.154167 & 41.640556 \\
\hline $8 / 28 / 09$ 14:10 & $14: 17$ & 5714230 & Normal & OK & Normal $\left(23^{\circ} \mathrm{C}\right)$ & Normal (56\%) & OK & OK & 127 & -88.181111 & 41.568611 \\
\hline $8 / 28 / 09$ 14:15 & $14: 22$ & 5714230 & Normal & OK & Normal $\left(23^{\circ} \mathrm{C}\right)$ & Normal (56\%) & OK & OK & 127 & -88.197778 & 41.493611 \\
\hline $8 / 28 / 0914: 20$ & $14: 22$ & 5714230 & Normal & OK & Normal $\left(23^{\circ} \mathrm{C}\right)$ & Normal (56\%) & OK & OK & 127 & -88.280000 & 41.462500 \\
\hline $8 / 28 / 09$ 14:25 & $14: 28$ & 5714230 & Normal & OK & Normal $\left(23^{\circ} \mathrm{C}\right)$ & Normal (56\%) & OK & $\mathrm{OK}$ & 127 & -88.371667 & 41.417500 \\
\hline $8 / 28 / 09$ 14:32 & $14: 39$ & 5714230 & Normal & OK & Normal $\left(23^{\circ} \mathrm{C}\right)$ & Normal (56\%) & OK & OK & 127 & -88.422778 & 41.392500 \\
\hline $8 / 28 / 09$ 14:35 & $14: 39$ & 5714230 & Normal & OK & Normal $\left(23^{\circ} \mathrm{C}\right)$ & Normal (56\%) & OK & OK & 127 & -88.422778 & 41.392500 \\
\hline $8 / 28 / 0914: 40$ & $14: 44$ & 5714230 & Normal & OK & Normal $\left(23^{\circ} \mathrm{C}\right)$ & Normal (56\%) & OK & OK & 127 & -88.422778 & 41.392500 \\
\hline $8 / 28 / 0914: 45$ & $14: 50$ & 5714230 & Normal & OK & Normal $\left(23^{\circ} \mathrm{C}\right)$ & Normal $(56 \%)$ & OK & OK & 127 & -88.461389 & 41.378056 \\
\hline $8 / 28 / 09$ 14:50 & $14: 56$ & 5714230 & Normal & OK & Normal $\left(23^{\circ} \mathrm{C}\right)$ & Normal (56\%) & OK & OK & 127 & -88.575000 & 41.376944 \\
\hline $8 / 28 / 09$ 14:55 & $15: 01$ & 5714230 & Normal & OK & Normal $\left(23^{\circ} \mathrm{C}\right)$ & Normal (56\%) & OK & OK & 127 & -88.717500 & 41.375833 \\
\hline $8 / 28 / 09$ 15:00 & 15:07 & 5714230 & Normal & OK & Normal $\left(23^{\circ} \mathrm{C}\right)$ & Normal (56\%) & OK & OK & 127 & -88.792500 & 41.376944 \\
\hline $8 / 28 / 09$ 15:05 & $15: 12$ & 5714230 & Normal & OK & Normal $\left(23^{\circ} \mathrm{C}\right)$ & Normal (56\%) & OK & OK & 127 & -88.901944 & 41.368611 \\
\hline $8 / 28 / 09$ 15:10 & $15: 12$ & 5714230 & Normal & OK & Normal $\left(23^{\circ} \mathrm{C}\right)$ & Normal $(56 \%)$ & OK & OK & 127 & -89.014444 & 41.367500 \\
\hline $8 / 28 / 09$ 15:15 & $15: 18$ & 5714230 & Normal & OK & Normal $\left(23^{\circ} \mathrm{C}\right)$ & Normal (56\%) & OK & OK & 127 & -89.058333 & 41.413333 \\
\hline $8 / 28 / 09$ 15:20 & $15: 23$ & 5714230 & Normal & OK & Normal $\left(23^{\circ} \mathrm{C}\right)$ & Normal (56\%) & OK & OK & 127 & -89.050833 & 41.460278 \\
\hline $8 / 28 / 0915: 25$ & $15: 29$ & 5714230 & Normal & OK & Normal $\left(23^{\circ} \mathrm{C}\right)$ & Normal $(56 \%)$ & OK & OK & 127 & -89.058333 & 41.375833 \\
\hline 8/28/09 15:30 & $15: 35$ & 5714230 & Normal & OK & Normal $\left(23^{\circ} \mathrm{C}\right)$ & Normal (56\%) & OK & OK & 127 & -89.125833 & 41.368611 \\
\hline $8 / 28 / 09$ 15:35 & $15: 40$ & 5714230 & Normal & OK & Normal $\left(23^{\circ} \mathrm{C}\right)$ & Normal (56\%) & OK & OK & 127 & -89.125833 & 41.368611 \\
\hline $8 / 28 / 09$ 15:40 & $15: 46$ & 5714230 & Normal & OK & Normal $\left(23^{\circ} \mathrm{C}\right)$ & Normal (56\%) & OK & OK & 127 & -89.125833 & 41.368611 \\
\hline $8 / 28 / 09$ 15:45 & $15: 51$ & 5714230 & Normal & OK & Normal $\left(23^{\circ} \mathrm{C}\right)$ & Normal $(56 \%)$ & OK & OK & 127 & -89.200000 & 41.364444 \\
\hline $8 / 28 / 09$ 15:50 & $15: 57$ & 5714230 & Normal & OK & Normal $\left(23^{\circ} \mathrm{C}\right)$ & Normal (56\%) & OK & OK & 127 & -89.240556 & 41.371667 \\
\hline $8 / 28 / 09$ 16:00 & $16: 03$ & 5714230 & Normal & OK & Normal $\left(23^{\circ} \mathrm{C}\right)$ & Normal (56\%) & OK & OK & 127 & -89.458333 & 41.400833 \\
\hline 8/28/09 16:05 & $16: 13$ & 5714230 & Normal & OK & Normal $\left(23^{\circ} \mathrm{C}\right)$ & Normal (56\%) & OK & OK & 127 & -89.558333 & 41.393611 \\
\hline $8 / 28 / 09$ 16:11 & $16: 14$ & 5714230 & Normal & OK & Normal $\left(23^{\circ} \mathrm{C}\right)$ & Normal (56\%) & OK & OK & 127 & -89.558333 & 41.393611 \\
\hline $8 / 28 / 09$ 16:15 & $16: 19$ & 5714230 & Normal & OK & Normal $\left(23^{\circ} \mathrm{C}\right)$ & Normal (56\%) & OK & OK & 127 & -89.558333 & 41.393611 \\
\hline $8 / 28 / 09$ 16:20 & $16: 25$ & 5714230 & Normal & OK & Normal $\left(23^{\circ} \mathrm{C}\right)$ & Normal (56\%) & OK & OK & 127 & -89.558333 & 41.393611 \\
\hline $8 / 28 / 09$ 16:25 & $16: 30$ & 5714230 & Normal & OK & Normal $\left(23^{\circ} \mathrm{C}\right)$ & Normal $(56 \%)$ & OK & OK & 127 & -89.623889 & 41.394722 \\
\hline $8 / 28 / 09$ 16:30 & $16: 36$ & 5714230 & Normal & OK & Normal $\left(23^{\circ} \mathrm{C}\right)$ & Normal $(56 \%)$ & OK & OK & 127 & -89.725833 & 41.387500 \\
\hline $8 / 28 / 09$ 16:35 & $16: 41$ & 5714230 & Normal & OK & Normal $\left(23^{\circ} \mathrm{C}\right)$ & Normal $(56 \%)$ & OK & OK & 127 & -89.834167 & 41.388333 \\
\hline $8 / 28 / 09$ 16:40 & $16: 47$ & 5714230 & Normal & OK & Normal $\left(23^{\circ} \mathrm{C}\right)$ & Normal (56\%) & OK & OK & 127 & -89.941667 & 41.416667 \\
\hline $8 / 28 / 09$ 16:45 & $16: 52$ & 5714230 & Normal & OK & Normal $\left(23^{\circ} \mathrm{C}\right)$ & Normal (56\%) & OK & OK & 127 & -90.050000 & 41.413333 \\
\hline $8 / 28 / 09$ 16:50 & $16: 52$ & 5714230 & Normal & OK & Normal $\left(23^{\circ} \mathrm{C}\right)$ & Normal (56\%) & OK & OK & 127 & -90.157222 & 41.430000 \\
\hline $8 / 28 / 09$ 16:55 & $16: 58$ & 5714230 & Normal & OK & Normal $\left(23^{\circ} \mathrm{C}\right)$ & Normal (56\%) & OK & OK & 127 & -90.267500 & 41.440556 \\
\hline $8 / 28 / 09$ 17:00 & 17:09 & 5714230 & Normal & OK & Normal $\left(23^{\circ} \mathrm{C}\right)$ & Normal (56\%) & OK & OK & 127 & -90.338333 & 41.476944 \\
\hline $8 / 28 / 09$ 17:05 & $17: 09$ & 5714230 & Normal & OK & Normal $\left(23^{\circ} \mathrm{C}\right)$ & Normal $(56 \%)$ & OK & OK & 127 & -90.334167 & 41.486389 \\
\hline $8 / 28 / 09$ 17:11 & $17: 15$ & 5714230 & Normal & OK & Normal $\left(23^{\circ} \mathrm{C}\right)$ & Normal $(56 \%)$ & OK & OK & 127 & -90.334167 & 41.486389 \\
\hline $8 / 28 / 09$ 17:15 & $17: 20$ & 5714230 & Normal & OK & Normal $\left(23^{\circ} \mathrm{C}\right)$ & Normal $(56 \%)$ & OK & OK & 127 & -90.334167 & 41.486389 \\
\hline $8 / 28 / 09$ 17:20 & $17: 26$ & 5714230 & Normal & OK & Normal $\left(23^{\circ} \mathrm{C}\right)$ & Normal (56\%) & OK & OK & 127 & -90.334167 & 41.486389 \\
\hline $8 / 28 / 0917: 25$ & $17: 31$ & 5714230 & Normal & OK & Normal $\left(23^{\circ} \mathrm{C}\right)$ & Normal (56\%) & OK & OK & 127 & -90.340556 & 41.511389 \\
\hline $8 / 28 / 09$ 17:30 & $17: 37$ & 5714230 & Normal & OK & Normal $\left(23^{\circ} \mathrm{C}\right)$ & Normal (56\%) & OK & OK & 127 & -90.234167 & 41.562500 \\
\hline $8 / 28 / 09$ 17:35 & $17: 42$ & 5714230 & Normal & OK & Normal $\left(23^{\circ} \mathrm{C}\right)$ & Normal (56\%) & OK & OK & 127 & -90.209167 & 41.581111 \\
\hline $8 / 28 / 0917: 40$ & $17: 42$ & 5714230 & Normal & OK & Normal $\left(23^{\circ} \mathrm{C}\right)$ & Normal (56\%) & $\mathrm{OK}$ & $\mathrm{OK}$ & 127 & -90.163333 & 41.642500 \\
\hline $8 / 28 / 09$ 17:45 & $17: 48$ & 5714230 & Normal & OK & Normal $\left(23^{\circ} \mathrm{C}\right)$ & Normal (56\%) & OK & OK & 127 & -90.080000 & 41.690556 \\
\hline $8 / 28 / 0917: 50$ & $17: 53$ & 5714230 & Normal & OK & Normal $\left(23^{\circ} \mathrm{C}\right)$ & Normal $(56 \%)$ & OK & OK & 127 & -89.9833333 & 41.718611 \\
\hline $8 / 28 / 0917: 55$ & $17: 59$ & 5714230 & Normal & OK & Normal $\left(25^{\circ} \mathrm{C}\right)$ & Normal $(55 \%)$ & OK & OK & 127 & -89.876944 & 41.744722 \\
\hline $8 / 28 / 09$ 18:00 & 18:04 & 5714230 & Normal & OK & Normal $\left(25^{\circ} \mathrm{C}\right)$ & Normal $(55 \%)$ & OK & OK & 127 & -89.775000 & 41.757222 \\
\hline $8 / 28 / 09$ 18:05 & $18: 10$ & 5714230 & Normal & OK & Normal $\left(25^{\circ} \mathrm{C}\right)$ & Normal (55\%) & OK & OK & 127 & -89.689444 & 41.750833 \\
\hline $8 / 28 / 09$ 18:10 & $18: 15$ & 5714230 & Normal & OK & Normal $\left(25^{\circ} \mathrm{C}\right)$ & Normal (55\%) & OK & OK & 127 & -89.689444 & 41.762500 \\
\hline $8 / 28 / 09$ 18:15 & $18: 21$ & 5714230 & Normal & OK & Normal $\left(25^{\circ} \mathrm{C}\right)$ & Normal (55\%) & OK & OK & 127 & -89.678056 & 41.750833 \\
\hline $8 / 28 / 0918: 20$ & $18: 26$ & 5714230 & Normal & OK & Normal $\left(25^{\circ} \mathrm{C}\right)$ & Normal (55\%) & OK & OK & 127 & -89.635278 & 41.759167 \\
\hline $8 / 28 / 09$ 18:25 & $18: 32$ & 5714230 & Normal & OK & Normal $\left(25^{\circ} \mathrm{C}\right)$ & Normal (55\%) & OK & OK & 127 & -89.5322222 & 41.789444 \\
\hline $8 / 28 / 09$ 18:30 & $18: 38$ & 5714230 & Normal & OK & Normal $\left(25^{\circ} \mathrm{C}\right)$ & Normal (55\%) & OK & $\mathrm{OK}$ & 127 & -89.432222 & 41.822778 \\
\hline $8 / 28 / 09$ 18:35 & $18: 38$ & 5714230 & Normal & OK & Normal $\left(25^{\circ} \mathrm{C}\right)$ & Normal $(55 \%)$ & OK & OK & 127 & -89.336389 & 41.822778 \\
\hline $8 / 28 / 0918: 40$ & $18: 43$ & 5714230 & Normal & OK & Normal $\left(25^{\circ} \mathrm{C}\right)$ & Normal (55\%) & OK & OK & 127 & -89.231111 & 41.850833 \\
\hline
\end{tabular}




\begin{tabular}{|c|c|c|c|c|c|c|c|c|c|c|c|}
\hline Event Time & at QC Hub & Tag ID & Tag Status & Seal & Temperature & Humidity & Shock & Battery & RSSI & Longitude & Latitude \\
\hline $8 / 28 / 09$ 18:45 & $18: 48$ & 5714230 & Normal & OK & Normal $\left(25^{\circ} \mathrm{C}\right)$ & Normal (55\%) & OK & OK & 127 & -89.125000 & 41.883333 \\
\hline 8/28/09 18:50 & $18: 54$ & 5714230 & Normal & OK & Normal $\left(25^{\circ} \mathrm{C}\right)$ & Normal (55\%) & OK & OK & 127 & -89.025000 & 41.904167 \\
\hline $8 / 28 / 09$ 18:55 & $19: 00$ & 5714230 & Normal & OK & Normal $\left(25^{\circ} \mathrm{C}\right)$ & Normal (55\%) & OK & OK & 127 & -88.913333 & 41.900000 \\
\hline 8/28/09 19:00 & 19:05 & 5714230 & Normal & OK & Normal $\left(25^{\circ} \mathrm{C}\right)$ & Normal (55\%) & OK & $\mathrm{OK}$ & 127 & -88.814444 & 41.900000 \\
\hline $8 / 28 / 09$ 19:05 & 19:11 & 5714230 & Normal & OK & Normal $\left(25^{\circ} \mathrm{C}\right)$ & Normal (55\%) & $\mathrm{OK}$ & $\mathrm{OK}$ & 127 & -88.739444 & 41.900000 \\
\hline 8/28/09 19:10 & 19:16 & 5714230 & Normal & OK & Normal $\left(25^{\circ} \mathrm{C}\right)$ & Normal $(55 \%)$ & OK & OK & 127 & -88.739444 & 41.900000 \\
\hline 8/28/09 19:15 & $19: 22$ & 5714230 & Normal & OK & Normal $\left(25^{\circ} \mathrm{C}\right)$ & Normal (54\%) & OK & OK & 127 & -88.737500 & 41.900000 \\
\hline $8 / 28 / 09$ 19:20 & $19: 27$ & 5714230 & Normal & OK & Normal $\left(25^{\circ} \mathrm{C}\right)$ & Normal (54\%) & OK & OK & 127 & -88.737500 & 41.900000 \\
\hline $8 / 28 / 09$ 19:25 & $19: 33$ & 5714230 & Normal & OK & Normal $\left(25^{\circ} \mathrm{C}\right)$ & Normal (54\%) & OK & OK & 127 & -88.639444 & 41.894722 \\
\hline 8/28/09 19:30 & $19: 38$ & 5714230 & Normal & OK & Normal $\left(25^{\circ} \mathrm{C}\right)$ & Normal (54\%) & OK & OK & 127 & -88.538333 & 41.859167 \\
\hline $8 / 28 / 09$ 19:35 & $19: 38$ & 5714230 & Normal & OK & Normal $\left(25^{\circ} \mathrm{C}\right)$ & Normal (54\%) & OK & OK & 127 & -88.448889 & 41.812500 \\
\hline $8 / 28 / 09$ 19:40 & $19: 44$ & 5714230 & Normal & OK & Normal $\left(25^{\circ} \mathrm{C}\right)$ & Normal (54\%) & OK & OK & 127 & -88.347778 & 41.794722 \\
\hline 8/28/09 19:45 & $19: 49$ & 5714230 & Normal & OK & Normal $\left(25^{\circ} \mathrm{C}\right)$ & Normal (54\%) & OK & OK & 127 & -88.261389 & 41.800000 \\
\hline $8 / 28 / 09$ 19:50 & $19: 55$ & 5714230 & Normal & OK & Normal $\left(25^{\circ} \mathrm{C}\right)$ & Normal $(54 \%)$ & OK & OK & 127 & -88.156111 & 41.806111 \\
\hline 8/28/09 19:55 & 20:00 & 5714230 & Normal & OK & Normal $\left(25^{\circ} \mathrm{C}\right)$ & Normal (54\%) & OK & OK & 127 & -88.062500 & 41.810278 \\
\hline $8 / 28 / 09$ 20:00 & $20: 06$ & 5714230 & Normal & OK & Normal $\left(25^{\circ} \mathrm{C}\right)$ & Normal (54\%) & OK & OK & 127 & -88.054167 & 41.807222 \\
\hline $8 / 28 / 09$ 20:05 & $20: 11$ & 5714230 & Normal & OK & Normal $\left(25^{\circ} \mathrm{C}\right)$ & Normal (54\%) & OK & OK & 127 & -88.054167 & 41.781111 \\
\hline $8 / 28 / 09$ 20:10 & $20: 22$ & 5714230 & Normal & OK & Normal $\left(25^{\circ} \mathrm{C}\right)$ & Normal (54\%) & OK & OK & 127 & -88.035278 & 41.732222 \\
\hline $8 / 28 / 0913: 40$ & $13: 44$ & 5714255 & Normal & OK & Normal $\left(23^{\circ} \mathrm{C}\right)$ & Normal (55\%) & OK & OK & 140 & -87.971667 & 41.732222 \\
\hline $8 / 28 / 09$ 13:45 & $13: 49$ & 5714255 & Normal & OK & Normal $\left(23^{\circ} \mathrm{C}\right)$ & Normal $(55 \%)$ & OK & $\mathrm{OK}$ & 140 & -87.971667 & 41.732222 \\
\hline $8 / 28 / 09$ 13:50 & $14: 00$ & 5714255 & Normal & OK & Normal $\left(23^{\circ} \mathrm{C}\right)$ & Normal (55\%) & OK & OK & 140 & -87.971667 & 41.732222 \\
\hline $8 / 28 / 09$ 13:55 & $14: 00$ & 5714255 & Normal & OK & Normal $\left(23^{\circ} \mathrm{C}\right)$ & Normal (55\%) & OK & OK & 140 & -87.971667 & 41.732222 \\
\hline $8 / 28 / 0914: 00$ & $14: 06$ & 5714255 & Normal & OK & Normal $\left(23^{\circ} \mathrm{C}\right)$ & Normal (55\%) & OK & OK & 140 & -88.068611 & 41.684167 \\
\hline $8 / 28 / 09$ 14:05 & $14: 11$ & 5714255 & Normal & OK & Normal $\left(23^{\circ} \mathrm{C}\right)$ & Normal (55\%) & OK & OK & 140 & -88.154167 & 41.640556 \\
\hline $8 / 28 / 0914: 10$ & $14: 17$ & 5714255 & Normal & OK & Normal $\left(23^{\circ} \mathrm{C}\right)$ & Normal (55\%) & OK & OK & 140 & -88.181111 & 41.568611 \\
\hline $8 / 28 / 0914: 15$ & $14: 22$ & 5714255 & Normal & OK & Normal $\left(23^{\circ} \mathrm{C}\right)$ & Normal (55\%) & OK & OK & 140 & -88.197778 & 41.493611 \\
\hline $8 / 28 / 0914: 20$ & $14: 22$ & 5714255 & Normal & OK & Normal $\left(23^{\circ} \mathrm{C}\right)$ & Normal (55\%) & OK & OK & 140 & -88.280000 & 41.462500 \\
\hline $8 / 28 / 0914: 25$ & $14: 28$ & 5714255 & Normal & OK & Normal $\left(23^{\circ} \mathrm{C}\right)$ & Normal $(55 \%)$ & OK & OK & 140 & -88.371667 & 41.417500 \\
\hline $8 / 28 / 0914: 32$ & $14: 39$ & 5714255 & Normal & OK & Normal $\left(23^{\circ} \mathrm{C}\right)$ & Normal (55\%) & OK & OK & 140 & -88.422778 & 41.392500 \\
\hline $8 / 28 / 0914: 35$ & $14: 39$ & 5714255 & Normal & OK & Normal $\left(23^{\circ} \mathrm{C}\right)$ & Normal (55\%) & OK & OK & 140 & -88.422778 & 41.392500 \\
\hline $8 / 28 / 0914: 40$ & $14: 44$ & 5714255 & Normal & OK & Normal $\left(23^{\circ} \mathrm{C}\right)$ & Normal (55\%) & OK & OK & 140 & -88.422778 & 41.392500 \\
\hline $8 / 28 / 0914: 45$ & $14: 50$ & 5714255 & Normal & OK & Normal $\left(23^{\circ} \mathrm{C}\right)$ & Normal (55\%) & OK & OK & 140 & -88.461389 & 41.378056 \\
\hline $8 / 28 / 0914: 50$ & $14: 56$ & 5714255 & Normal & OK & Normal $\left(23^{\circ} \mathrm{C}\right)$ & Normal (55\%) & OK & OK & 140 & -88.575000 & 41.376944 \\
\hline $8 / 28 / 0914: 55$ & 15:01 & 5714255 & Normal & OK & Normal $\left(23^{\circ} \mathrm{C}\right)$ & Normal $(54 \%)$ & OK & OK & 140 & -88.717500 & 41.375833 \\
\hline $8 / 28 / 09$ 15:00 & $15: 07$ & 5714255 & Normal & OK & Normal $\left(23^{\circ} \mathrm{C}\right)$ & Normal $(54 \%)$ & OK & $\mathrm{OK}$ & 140 & -88.792500 & 41.376944 \\
\hline $8 / 28 / 09$ 15:05 & $15: 12$ & 5714255 & Normal & OK & Normal $\left(23^{\circ} \mathrm{C}\right)$ & Normal $(54 \%)$ & OK & OK & 140 & -88.901944 & 41.368611 \\
\hline $8 / 28 / 09$ 15:10 & $15: 12$ & 5714255 & Normal & OK & Normal $\left(23^{\circ} \mathrm{C}\right)$ & Normal (54\%) & OK & OK & 140 & -89.014444 & 41.367500 \\
\hline $8 / 28 / 09$ 15:15 & $15: 18$ & 5714255 & Normal & OK & Normal $\left(23^{\circ} \mathrm{C}\right)$ & Normal (54\%) & OK & OK & 140 & -89.058333 & 41.413333 \\
\hline $8 / 28 / 09$ 15:20 & $15: 23$ & 5714255 & Normal & OK & Normal $\left(23^{\circ} \mathrm{C}\right)$ & Normal (54\%) & OK & OK & 140 & -89.050833 & 41.460278 \\
\hline $8 / 28 / 09$ 15:25 & $15: 29$ & 5714255 & Normal & OK & Normal $\left(23^{\circ} \mathrm{C}\right)$ & Normal (54\%) & OK & OK & 140 & -89.058333 & 41.375833 \\
\hline $8 / 28 / 09$ 15:30 & $15: 35$ & 5714255 & Normal & OK & Normal $\left(23^{\circ} \mathrm{C}\right)$ & Normal (54\%) & OK & OK & 140 & -89.125833 & 41.368611 \\
\hline $8 / 28 / 09$ 15:35 & $15: 40$ & 5714255 & Normal & OK & Normal $\left(23^{\circ} \mathrm{C}\right)$ & Normal (54\%) & OK & OK & 140 & -89.125833 & 41.368611 \\
\hline $8 / 28 / 09$ 15:40 & $15: 46$ & 5714255 & Normal & OK & Normal $\left(23^{\circ} \mathrm{C}\right)$ & Normal (54\%) & OK & OK & 140 & -89.125833 & 41.368611 \\
\hline $8 / 28 / 0915: 45$ & $15: 51$ & 5714255 & Normal & OK & Normal $\left(23^{\circ} \mathrm{C}\right)$ & Normal (54\%) & OK & OK & 140 & -89.200000 & 41.364444 \\
\hline $8 / 28 / 0915: 50$ & $15: 57$ & 5714255 & Normal & OK & Normal $\left(23^{\circ} \mathrm{C}\right)$ & Normal (54\%) & OK & OK & 140 & -89.240556 & 41.371667 \\
\hline $8 / 28 / 09$ 16:00 & $16: 03$ & 5714255 & Normal & OK & Normal $\left(23^{\circ} \mathrm{C}\right)$ & Normal (54\%) & OK & OK & 140 & -89.458333 & 41.400833 \\
\hline $8 / 28 / 0916: 05$ & $16: 13$ & 5714255 & Normal & OK & Normal $\left(23^{\circ} \mathrm{C}\right)$ & Normal (54\%) & OK & OK & 140 & -89.558333 & 41.393611 \\
\hline $8 / 28 / 09$ 16:09 & $16: 13$ & 5714255 & Alarm & Open & Normal $\left(23^{\circ} \mathrm{C}\right)$ & Normal $(52 \%)$ & OK & OK & 140 & -89.558333 & 41.393611 \\
\hline $8 / 28 / 0916: 11$ & $16: 14$ & 5714255 & Normal & OK & Normal $\left(23^{\circ} \mathrm{C}\right)$ & Normal $(54 \%)$ & OK & $\mathrm{OK}$ & 140 & -89.558333 & 41.393611 \\
\hline $8 / 28 / 0916: 15$ & $16: 19$ & 5714255 & Normal & OK & Normal $\left(23^{\circ} \mathrm{C}\right)$ & Normal (54\%) & OK & OK & 140 & -89.558333 & 41.393611 \\
\hline $8 / 28 / 0916: 20$ & $16: 25$ & 5714255 & Normal & OK & Normal $\left(23^{\circ} \mathrm{C}\right)$ & Normal (54\%) & OK & OK & 140 & -89.558333 & 41.393611 \\
\hline $8 / 28 / 0916: 25$ & $16: 30$ & 5714255 & Normal & OK & Normal $\left(23^{\circ} \mathrm{C}\right)$ & Normal (54\%) & OK & OK & 140 & -89.623889 & 41.394722 \\
\hline $8 / 28 / 09$ 16:30 & $16: 36$ & 5714255 & Normal & OK & Normal $\left(23^{\circ} \mathrm{C}\right)$ & Normal (54\%) & OK & OK & 140 & -89.725833 & 41.387500 \\
\hline $8 / 28 / 09$ 16:35 & $16: 41$ & 5714255 & Normal & OK & Normal $\left(23^{\circ} \mathrm{C}\right)$ & Normal (54\%) & OK & OK & 140 & -89.834167 & 41.388333 \\
\hline $8 / 28 / 0916: 40$ & $16: 47$ & 5714255 & Normal & OK & Normal $\left(23^{\circ} \mathrm{C}\right)$ & Normal (54\%) & OK & OK & 140 & -89.941667 & 41.416667 \\
\hline $8 / 28 / 09$ 16:45 & $16: 52$ & 5714255 & Normal & OK & Normal $\left(23^{\circ} \mathrm{C}\right)$ & Normal $(54 \%)$ & OK & OK & 140 & -90.050000 & 41.413333 \\
\hline $8 / 28 / 09$ 16:50 & $16: 52$ & 5714255 & Normal & OK & Normal $\left(23^{\circ} \mathrm{C}\right)$ & Normal (54\%) & OK & OK & 140 & -90.157222 & 41.430000 \\
\hline $8 / 28 / 0916: 55$ & $16: 58$ & 5714255 & Normal & OK & Normal $\left(23^{\circ} \mathrm{C}\right)$ & Normal (54\%) & OK & OK & 140 & -90.267500 & 41.440556 \\
\hline $8 / 28 / 09$ 17:00 & $17: 09$ & 5714255 & Normal & OK & Normal $\left(23^{\circ} \mathrm{C}\right)$ & Normal (54\%) & OK & OK & 140 & -90.338333 & 41.476944 \\
\hline $8 / 28 / 09$ 17:05 & 17:09 & 5714255 & Normal & OK & Normal $\left(23^{\circ} \mathrm{C}\right)$ & Normal (54\%) & OK & OK & 140 & -90.334167 & 41.486389 \\
\hline $8 / 28 / 0917: 11$ & $17: 15$ & 5714255 & Normal & OK & Normal $\left(23^{\circ} \mathrm{C}\right)$ & Normal (54\%) & OK & OK & 140 & -90.334167 & 41.486389 \\
\hline $8 / 28 / 09$ 17:15 & $17: 20$ & 5714255 & Normal & OK & Normal $\left(23^{\circ} \mathrm{C}\right)$ & Normal (54\%) & OK & $\mathrm{OK}$ & 140 & -90.334167 & 41.486389 \\
\hline $8 / 28 / 0917: 20$ & $17: 26$ & 5714255 & Normal & OK & Normal $\left(23^{\circ} \mathrm{C}\right)$ & Normal $(54 \%)$ & OK & $\mathrm{OK}$ & 140 & -90.334167 & 41.486389 \\
\hline $8 / 28 / 09$ 17:25 & $17: 31$ & 5714255 & Normal & OK & Normal $\left(23^{\circ} \mathrm{C}\right)$ & Normal $(54 \%)$ & OK & OK & 140 & -90.340556 & 41.511389 \\
\hline $8 / 28 / 09$ 17:30 & $17: 37$ & 5714255 & Normal & OK & Normal $\left(23^{\circ} \mathrm{C}\right)$ & Normal (54\%) & OK & OK & 140 & -90.234167 & 41.562500 \\
\hline $8 / 28 / 09$ 17:35 & $17: 42$ & 5714255 & Normal & OK & Normal $\left(23^{\circ} \mathrm{C}\right)$ & Normal (54\%) & OK & OK & 140 & -90.209167 & 41.581111 \\
\hline $8 / 28 / 09$ 17:40 & $17: 42$ & 5714255 & Normal & OK & Normal $\left(23^{\circ} \mathrm{C}\right)$ & Normal (54\%) & OK & OK & 140 & -90.163333 & 41.642500 \\
\hline $8 / 28 / 09$ 17:45 & $17: 48$ & 5714255 & Normal & OK & Normal $\left(23^{\circ} \mathrm{C}\right)$ & Normal (54\%) & OK & OK & 140 & -90.080000 & 41.690556 \\
\hline $8 / 28 / 09$ 17:50 & $17: 53$ & 5714255 & Normal & OK & Normal $\left(23^{\circ} \mathrm{C}\right)$ & Normal (54\%) & OK & OK & 140 & -89.983333 & 41.718611 \\
\hline $8 / 28 / 09$ 17:55 & $17: 59$ & 5714255 & Normal & OK & Normal $\left(26^{\circ} \mathrm{C}\right)$ & Normal (54\%) & OK & OK & 140 & -89.876944 & 41.744722 \\
\hline $8 / 28 / 09$ 18:00 & $18: 04$ & 5714255 & Normal & OK & Normal $\left(26^{\circ} \mathrm{C}\right)$ & Normal (54\%) & OK & OK & 140 & -89.775000 & 41.757222 \\
\hline $8 / 28 / 09$ 18:05 & $18: 10$ & 5714255 & Normal & OK & Normal $\left(26^{\circ} \mathrm{C}\right)$ & Normal (54\%) & OK & OK & 140 & -89.689444 & 41.750833 \\
\hline $8 / 28 / 0918: 10$ & $18: 15$ & 5714255 & Normal & OK & Normal $\left(26^{\circ} \mathrm{C}\right)$ & Normal (54\%) & OK & OK & 140 & -89.689444 & 41.762500 \\
\hline 8/28/09 18:15 & $18: 21$ & 5714255 & Normal & OK & Normal $\left(26^{\circ} \mathrm{C}\right)$ & Normal (54\%) & OK & $\mathrm{OK}$ & 140 & -89.678056 & 41.750833 \\
\hline $8 / 28 / 0918: 20$ & $18: 26$ & 5714255 & Normal & OK & Normal $\left(26^{\circ} \mathrm{C}\right)$ & Normal (54\%) & OK & OK & 140 & -89.635278 & 41.759167 \\
\hline $8 / 28 / 0918: 25$ & $18: 32$ & 5714255 & Normal & OK & Normal $\left(26^{\circ} \mathrm{C}\right)$ & Normal (54\%) & OK & $\mathrm{OK}$ & 140 & -89.532222 & 41.789444 \\
\hline $8 / 28 / 09$ 18:30 & $18: 38$ & 5714255 & Normal & OK & Normal $\left(26^{\circ} \mathrm{C}\right)$ & Normal (54\%) & OK & OK & 140 & -89.432222 & 41.822778 \\
\hline $8 / 28 / 0918: 35$ & $18: 38$ & 5714255 & Normal & OK & Normal $\left(26^{\circ} \mathrm{C}\right)$ & Normal $(54 \%)$ & OK & OK & 140 & -89.336389 & 41.822778 \\
\hline $8 / 28 / 0918: 40$ & $18: 43$ & 5714255 & Normal & OK & Normal $\left(26^{\circ} \mathrm{C}\right)$ & Normal (54\%) & OK & OK & 140 & -89.231111 & 41.850833 \\
\hline 8/28/09 18:45 & $18: 48$ & 5714255 & Normal & OK & Normal $\left(26^{\circ} \mathrm{C}\right)$ & Normal (53\%) & OK & OK & 140 & -89.125000 & 41.883333 \\
\hline $8 / 28 / 09$ 18:50 & $18: 54$ & 5714255 & Normal & OK & Normal $\left(26^{\circ} \mathrm{C}\right)$ & Normal (53\%) & $\mathrm{OK}$ & $\mathrm{OK}$ & 140 & -89.025000 & 41.904167 \\
\hline
\end{tabular}




\begin{tabular}{|c|c|c|c|c|c|c|c|c|c|c|c|}
\hline Event Time & at QC Hub & Tag ID & Tag Status & Seal & Temperature & Humidity & Shock & Battery & RSSI & Longitude & Latitude \\
\hline 8/28/09 18:55 & 19:00 & 5714255 & Normal & OK & Normal $\left(26^{\circ} \mathrm{C}\right)$ & Normal $(53 \%)$ & OK & OK & 140 & -88.913333 & 41.900000 \\
\hline 8/28/09 19:00 & 19:05 & 5714255 & Normal & OK & Normal $\left(26^{\circ} \mathrm{C}\right)$ & Normal (53\%) & OK & OK & 140 & -88.814444 & 41.900000 \\
\hline $8 / 28 / 09$ 19:05 & 19:11 & 5714255 & Normal & OK & Normal $\left(26^{\circ} \mathrm{C}\right)$ & Normal $(53 \%)$ & OK & OK & 140 & -88.739444 & 41.900000 \\
\hline 8/28/09 19:10 & 19:16 & 5714255 & Normal & OK & Normal $\left(26^{\circ} \mathrm{C}\right)$ & Normal (53\%) & OK & OK & 140 & -88.739444 & 41.900000 \\
\hline 8/28/09 19:15 & 19:22 & 5714255 & Normal & OK & Normal $\left(26^{\circ} \mathrm{C}\right)$ & Normal $(52 \%)$ & OK & OK & 140 & -88.737500 & 41.900000 \\
\hline $8 / 28 / 09$ 19:20 & 19:27 & 5714255 & Normal & OK & Normal $\left(26^{\circ} \mathrm{C}\right)$ & Normal $(52 \%)$ & OK & OK & 140 & -88.737500 & 41.900000 \\
\hline $8 / 28 / 09$ 19:25 & 19:33 & 5714255 & Normal & OK & Normal $\left(26^{\circ} \mathrm{C}\right)$ & Normal $(52 \%)$ & OK & OK & 140 & -88.639444 & 41.894722 \\
\hline 8/28/09 19:30 & 19:38 & 5714255 & Normal & OK & Normal $\left(26^{\circ} \mathrm{C}\right)$ & Normal $(52 \%)$ & OK & OK & 140 & -88.538333 & 41.859167 \\
\hline 8/28/09 19:35 & $19: 38$ & 5714255 & Normal & OK & Normal $\left(26^{\circ} \mathrm{C}\right)$ & Normal $(52 \%)$ & OK & OK & 140 & -88.448889 & 41.812500 \\
\hline $8 / 28 / 09$ 19:40 & $19: 44$ & 5714255 & Normal & OK & Normal $\left(26^{\circ} \mathrm{C}\right)$ & Normal $(52 \%)$ & OK & OK & 140 & -88.347778 & 41.794722 \\
\hline $8 / 28 / 09$ 19:45 & $19: 49$ & 5714255 & Normal & OK & Normal $\left(26^{\circ} \mathrm{C}\right)$ & Normal $(52 \%)$ & OK & OK & 140 & -88.261389 & 41.800000 \\
\hline 8/28/09 19:50 & 19:55 & 5714255 & Normal & OK & Normal $\left(26^{\circ} \mathrm{C}\right)$ & Normal $(52 \%)$ & OK & OK & 140 & -88.156111 & 41.806111 \\
\hline 8/28/09 19:55 & 20:00 & 5714255 & Normal & OK & Normal $\left(26^{\circ} \mathrm{C}\right)$ & Normal $(52 \%)$ & OK & OK & 140 & -88.062500 & 41.810278 \\
\hline $8 / 28 / 0920: 00$ & 20:06 & 5714255 & Normal & OK & Normal $\left(26^{\circ} \mathrm{C}\right)$ & Normal $(52 \%)$ & OK & OK & 140 & -88.054167 & 41.807222 \\
\hline 8/28/09 20:05 & 20:11 & 5714255 & Normal & OK & Normal $\left(26^{\circ} \mathrm{C}\right)$ & Normal (53\%) & OK & OK & 140 & -88.054167 & 41.781111 \\
\hline $8 / 28 / 09$ 20:10 & $20: 22$ & 5714255 & Normal & OK & Normal $\left(26^{\circ} \mathrm{C}\right)$ & Normal (53\%) & OK & OK & 140 & -88.035278 & 41.732222 \\
\hline
\end{tabular}




\section{Appendix D: Geographic Information System (GIS) Reports}

Three GIS reports were generated during this demo. They are shown in the following:

\section{GIS Report for DEMO Vehicle}

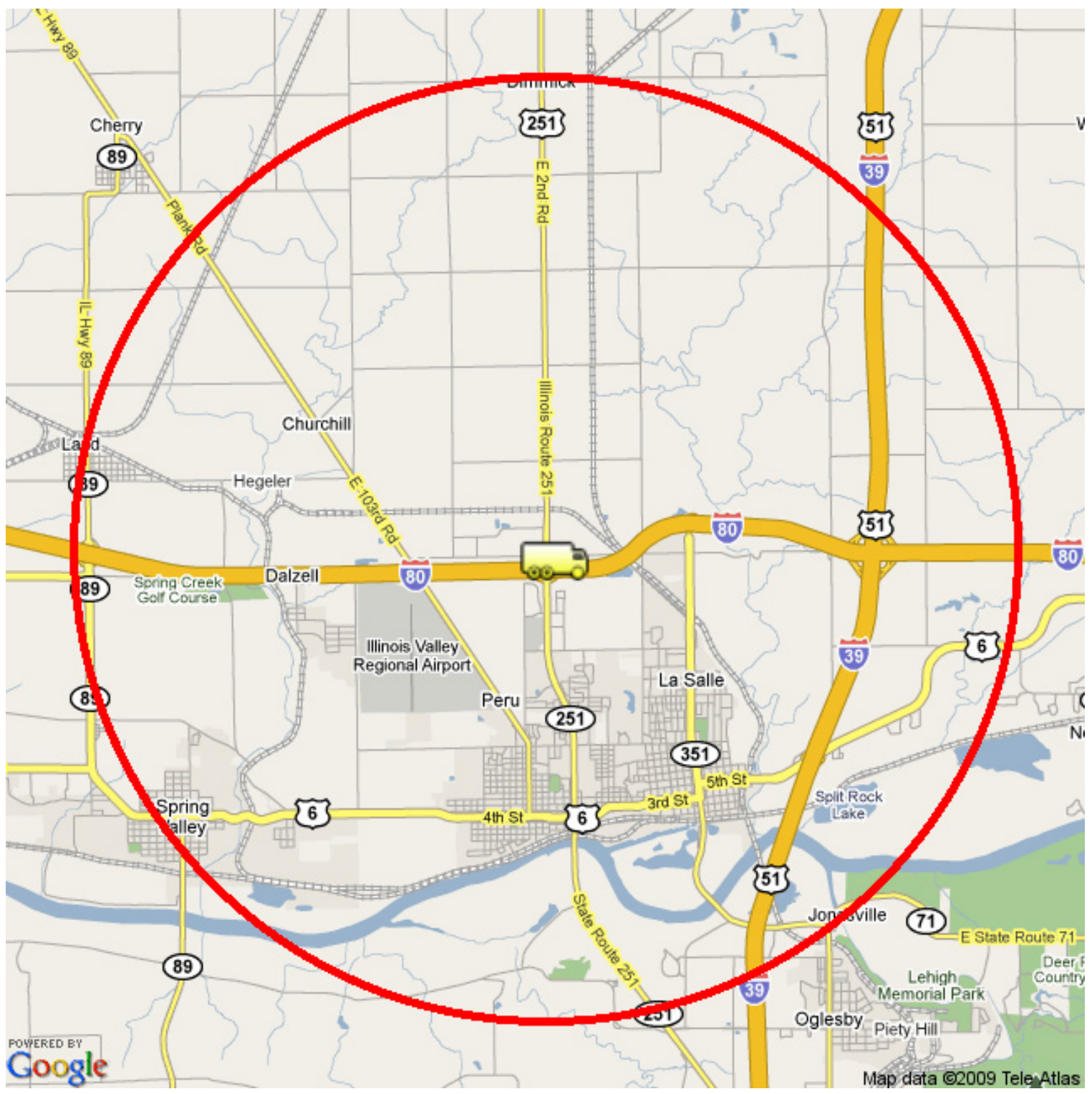




\section{EMS Facilities within 5 Miles of DEMO Vehicle (41.368611,-89.125833) (As of 8/28/09 15:36 GMT)}

Name: PERU VOLUNTEER AMBULANCE SERVICE

Description: AMBULANCE SERVICES, AIR OR GROUND

Phone: 815-223-9111

Address: 111 5TH STREET, PERU, IL 61354

County: LA SALLE

Directions: LOCATED ON NORTH SIDE OF 5TH STREET (UNITED STATES HIGHWAY 6) .06 MILES EAST OF ORLEANS STREET.

Approximate Distance from DEMO Vehicle: 2.88 miles

Name: DALZELL FIRE DEPARTMENT

Description: AMBULANCE AND FIRE SERVICE COMBINED

Phone: 815-663-7371

Address: LUCY STREET, DALZELL, IL 61320

County: BUREAU

Directions: LOCATED ON EAST SIDE OF LUCY STREET BETWEEN SCOTT STREET AND SAUNDERS STREET.

Approximate Distance from DEMO Vehicle: 2.49 miles

Name: LASALLE FIRE AND EMERGENCY SERVICES

Description: AMBULANCE AND FIRE SERVICE COMBINED

Phone: 815-223-0834

Address: 1227 5TH STREET, LASALLE, IL 61301

County: LA SALLE

Directions: LOCATED ON THE NW CORNER OF 5TH ST AND STERLING ST.

Approximate Distance from DEMO Vehicle: 3.12 miles

Please call or send your comments, feedback, and suggestions to:

Dr. Yung Liu (630) 252-5127 yyliu@anl.gov

or Dr. James Shuler (301) 903-5513 james.shuler@em.doe.gov 
If Fnvironanental Managenent

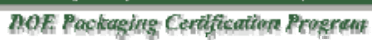

\section{Packaging RFID Tracking System}

Nos

\section{GIS Report for DEMO Vehicle}

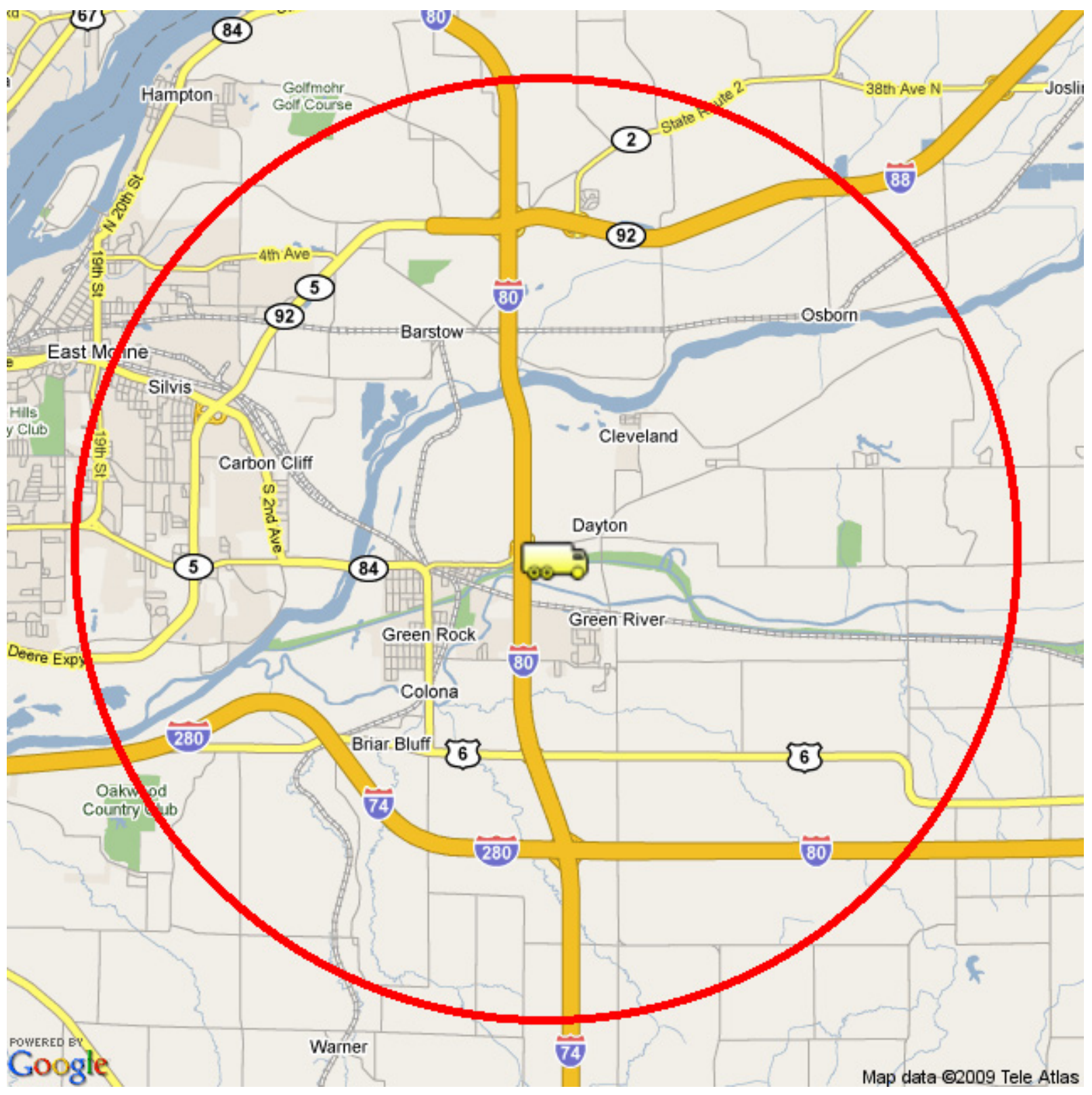




\section{EMS Facilities within 10 Miles of DEMO Vehicle (41.486389,-90.334167) (As of 8/28/09 17:17 GMT)}

Name: MOLINE FIRE DEPARTMENT STATION 3

Description: AMBULANCE AND FIRE SERVICE COMBINED

Phone: 309-797-0713

Address: 4700 38TH AVENUE, MOLINE, IL 61265

County: ROCK ISLAND

Directions: SOUTH SIDE OF 38TH AVENUE BETWEEN 48TH STREET AND 47TH STREET

Approximate Distance from DEMO Vehicle: 7.2 miles

Name: SILVIS FIRE DEPARTMENT

Description: AMBULANCE AND FIRE SERVICE COMBINED

Phone: 309-792-9553

Address: 1040 1ST AVENUE, SILVIS, IL 61282

County: ROCK ISLAND

Directions: ON THE SOUTH SIDE OF 1ST AVE BETWEEN 10TH ST AND 11TH ST.

Approximate Distance from DEMO Vehicle: 4.47 miles

Name: MOLINE FIRE DEPARTMENT

Description: AMBULANCE AND FIRE SERVICE COMBINED

Phone: 309-797-0401

Address: 1630 8TH AVENUE, MOLINE, IL 61265

County: ROCK ISLAND

Directions: SOUTH SIDE OF 8TH AVENUE BETWEEN 16TH STREET AND 17TH STREET

Approximate Distance from DEMO Vehicle: 9.3 miles

Name: ORION AMBULANCE SERVICE

Description: AMBULANCE SERVICES, AIR OR GROUND

Phone: $309-526-3365$

Address: $50111 \mathrm{TH}$ AVENUE, ORION, IL 61273

County: HENRY

Directions: LOCATED ON NORTH SIDE OF 11TH AVENUE BETWEEN 5TH STREET AND 6TH

STREET.

Approximate Distance from DEMO Vehicle: 9.46 miles

Name: GENESEO FIRE PROTECTION DISTRICT/AMBULANCE SERVICE

Description: AMBULANCE AND FIRE SERVICE COMBINED

Phone: $309-944-5544$

Address: 124 EAST 2ND STREET, GENESEO, IL 61254

County: HENRY

Directions: LOCATED ON SOUTH SIDE OF EAST 2ND STREET BETWEEN SOUTH OAKWOOD AVENUE AND SOUTH STATE STREET.

Approximate Distance from DEMO Vehicle: 9.62 miles

Name: EAST MOLINE FIRE DEPARTMENT

Description: AMBULANCE AND FIRE SERVICE COMBINED

Phone: 309-752-1503

Address: 1523 MORTON DRIVE, EAST MOLINE, IL 61244

County: ROCK ISLAND

Directions: NORTH SIDE OF MORTON DRIVE APPROXIMATELY .05 MILES WEST FROM THE

INTERSECTION OF 19TH STREET AND MORTON DRIVE

Approximate Distance from DEMO Vehicle: 5.45 miles

Name: COAL VALLEY FIRE PROTECTION DISTRICT

Description: AMBULANCE AND FIRE SERVICE COMBINED

Phone: 309-799-5534

Address: 103 WEST 18TH AVENUE, COAL VALLEY, IL 61240 
County: ROCK ISLAND

Directions: SOUTH SIDE OF WEST 18TH AVENUE APPROXIMATLEY .02 MILES WEST FROM THE INTERSECTION OF 1ST STREET AND WEST 18TH AVENUE.

Approximate Distance from DEMO Vehicle: 7.5 miles

Name: BETTENDORF FIRE DEPARTMENT 4

Description: AMBULANCE AND FIRE SERVICE COMBINED

Phone: 563-344-7160

Address: 5002 CROW CREEK ROAD, BETTENDORF, IA 52722

County: SCOTT

Directions: LOCATED ON THE NORTHEAST CORNER OF MIDDLE RD AND CROW CREEK RD. Approximate Distance from DEMO Vehicle: 8.79 miles

Name: BETTENDORF FIRE DEPARTMENT STATION 2

Description: AMBULANCE AND FIRE SERVICE COMBINED

Phone: 563-344-4148

Address: 1933 SPRUCE HILLS DRIVE, BETTENDORF, IA 52722

County: SCOTT

Directions: LOCATED ON THE SOUTHWEST SIDE OF SPRUCE HILLS DR, .01 MILES SOUTHEAST OF S HAMPTON DR.

Approximate Distance from DEMO Vehicle: 9.93 miles

Name: BETTENDORF FIRE DEPARTMENT STATION 1

Description: AMBULANCE AND FIRE SERVICE COMBINED

Phone: 563-344-4052

Address: 1609 STATE STREET, BETTENDORF, IA 52722

County: SCOTT

Directions: LOCATED ON THE SOUTH SIDE OF STATE ST, BETWEEN 16TH ST AND 17TH ST.

Approximate Distance from DEMO Vehicle: 9.47 miles

Name: LECLAIRE FIRE AND RESCUE DEPT

Description: FIRE AND RESCUE SERVICE

Phone: 563-289-5257

Address: $201 \mathrm{~N}$ 15TH ST, LE CLAIRE, IA 52753

County: SCOTT

Directions: NORTHEAST CORNER OF JONES ST AND N 15TH ST INTERSECTION

Approximate Distance from DEMO Vehicle: 7.93 miles

Name: RIVERDALE FIRE DEPARTMENT

Description: AMBULANCE AND FIRE SERVICE COMBINED

Phone: 563-355-2211

Address: 110 MANOR DRIVE, RIVERDALE, IA 52722

County: SCOTT

Directions: LOCATED IN WEST CORNER OF MANOR DRIVE AND STATE STREET.

Approximate Distance from DEMO Vehicle: 7.72 miles

Name: BETTENDORF FIRE DEPARTMENT \#3 AMBULANCE

Description: AMBULANCE AND FIRE SERVICE COMBINED

Phone: 563-332-4355

Address: 24495 EAST VALLEY DRIVE, PLEASANT VALLEY, IA 52722

County: SCOTT

Directions: S SIDE OF E VALLEY DR, BETWEEN 23RD AVE AND N SPENCER RD

Approximate Distance from DEMO Vehicle: 7.4 miles 
Please call or send your comments, feedback, and suggestions to:

Dr. Yung Liu (630) 252-5127 yyliu@anl.gov

or Dr. James Shuler (301) 903-5513 james.shuler@em.doe.gov

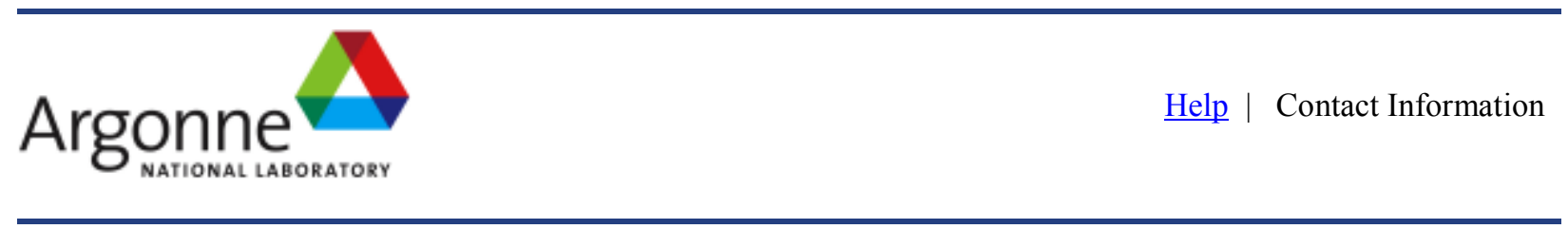

Current time: Fri, 28 Aug 2009 17:17:50 UTC 
I Envipownental Management

\section{GIS Report for DEMO Vehicle}

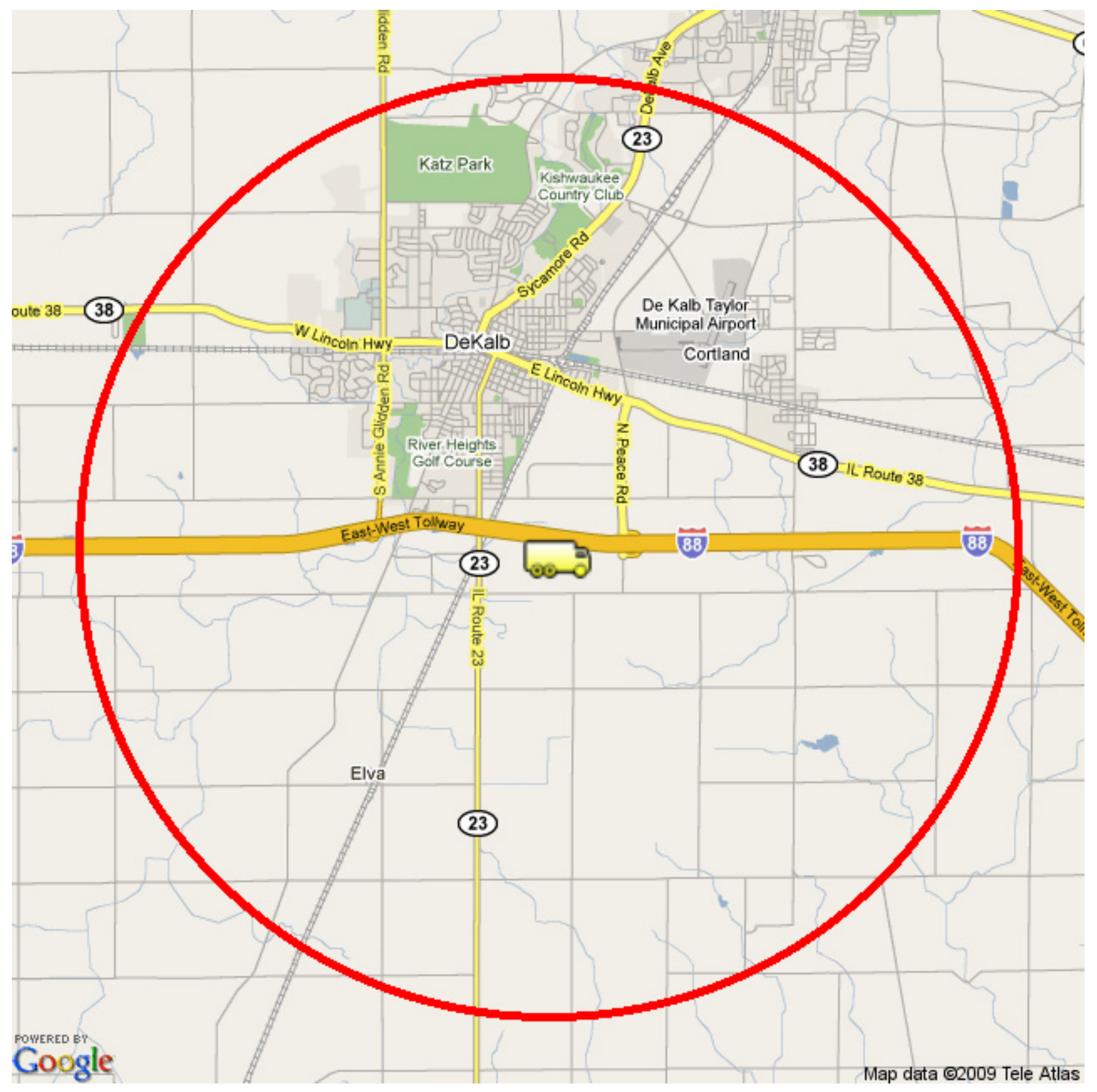




\section{(As of 8/28/09 19:13 GMT)}

Name: RIDGE AMBULANCE SERVICE

Description: AMBULANCE SERVICES, AIR OR GROUND

Phone: 815-756-3424

Address: 1306 SOUTH 4TH STREET, DEKALB, IL 60115

County: DEKALB

Directions: LOCATED ON THE SOUTHEAST CORNER OF TYLER STREET AND SOUTH 4TH STREET.

Approximate Distance from DEMO Vehicle: 1.35 miles

Name: HINCKLEY FIRE PROTECTION DISTRICT

Description: AMBULANCE AND FIRE SERVICE COMBINED

Phone: $815-286-7711$

Address: 911 NORTH SYCAMORE STREET, HINCKLEY, IL 60520

County: DEKALB

Directions: ENTITY LOCATED APPROX 0.14 MILES NORTH OF INTERSECTION OF NORTH

SYCAMORE STREET AND WEST AMIE AVENUE.

Approximate Distance from DEMO Vehicle: 9.78 miles

Name: SYCAMORE FIRE DEPARTMENT AMBULANCE

Description: AMBULANCE AND FIRE SERVICE COMBINED

Phone: 815-895-4514

Address: 535 DEKALB AVENUE, SYCAMORE, IL 60178

County: DEKALB

Directions: ENTITY LOCATED ON NORTHWEST CORNER OF SOUTH SACREMENTO STREET AND

DEKALB AVENUE.

Approximate Distance from DEMO Vehicle: 6.56 miles

Name: MALTA FIRE PROTECTION DISTRICT AMBULANCE

Description: AMBULANCE AND FIRE SERVICE COMBINED

Phone: 815-825-2067

Address: 308 EAST JEFFERSON STREET, MALTA, IL 60150

County: DEKALB

Directions: ENTITY LOCATED ON THE NORTHWEST CORNER OF EAST JEFFERSON STREET AND

NORTH 4TH STREET.

Approximate Distance from DEMO Vehicle: 6.62 miles

Name: DEKALB FIRE DEPARTMENT

Description: AMBULANCE AND FIRE SERVICE COMBINED

Phone: 815-748-8460

Address: 700 PINE STREET, DEKALB, IL 60115

County: DEKALB

Directions: ENTITY LOCATED ON THE SOUTH CORNER OF NORTH 7TH STREET AND PINE STREET.

Approximate Distance from DEMO Vehicle: 2.16 miles

Please call or send your comments, feedback, and suggestions to:

Dr. Yung Liu (630) 252-5127 yyliu@anl.gov

or Dr. James Shuler (301) 903-5513 james.shuler@em.doe.gov

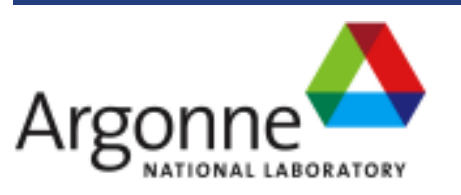

$\underline{\text { Help }}$ | Contact Information

Current time: Fri, 28 Aug 2009 19:14:12 UTC 


Argonne

Decision and Information Sciences Division

Argonne National Laboratory

9700 South Cass Avenue, Bldg. 900

Argonne, IL 60439-4867

www.anl.gov 